DIVERSITY AND CHARACTERIZATION OF NOVEL CYTOCHROME P450 2

GENES IN THE MARINE TELEOST FUNDULUS HETEROCLITUS

\author{
by \\ Marjorie Frances Oleksiak \\ S. B. Biology \\ Massachusetts Institute of Technology, Cambridge, MA 1989 \\ B. A. Mathematics \\ Wellesley College, Wellesley, MA 1988
}

SUBMITTED IN PARTIAL FULFILLMENT OF THE REQUIREMENTS FOR THE

DEGREE OF DOCTOR OF PHILOSOPHY

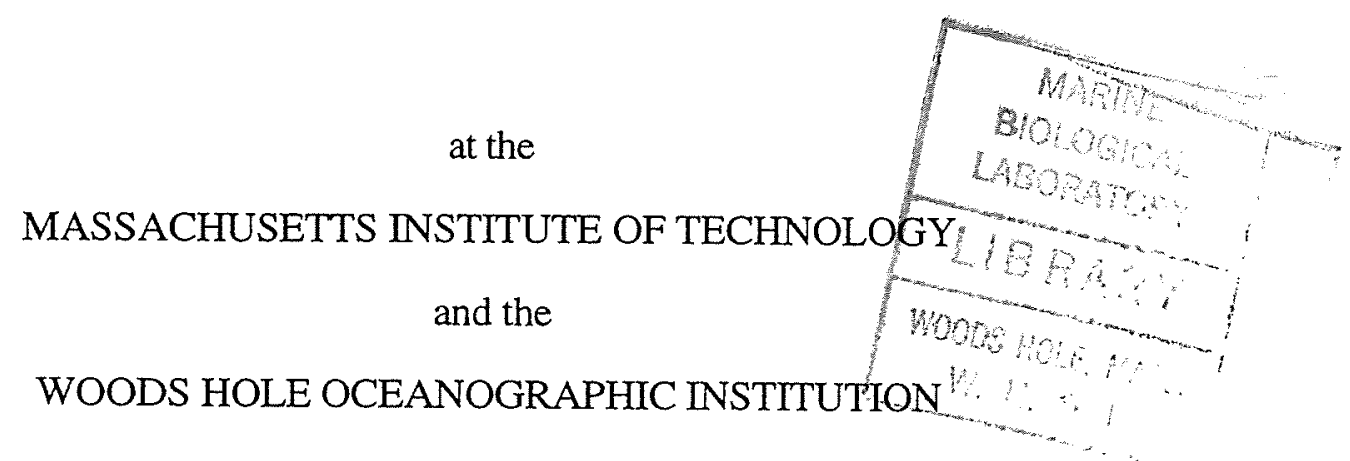

September, 1997

(c) Marjorie F. Oleksiak, 1997

All right reserved.

The author hereby grants to M.I.T. and,W.H.O.I. permission to reproduce and distribute copies of this thesis document in whole or in part.

Signature of Author

Joint Program in Biological Oceanography

Massachusetts Institute of Technologv/Woods Hole Oceanographic Institution

Certified by

John J. Stedeman, Dhesis Subervisor

Accepted by

Mark E. Hahn, Chair

Joint Committe for Biological Oceanography,

Massachusetts Institute of Technology/Woods Hole Oceanographic Institution 


\title{
DIVERSITY AND CHARACTERIZATION OF NOVEL CYTOCHROME P450 2 GENES IN THE MARINE TELEOST FUNDULUS HETEROCLITUS
}

\author{
by \\ Marjorie Frances Oleksiak \\ submitted in partial fulfillment of the requirements for the degree of \\ Doctor of Philosophy
}

\begin{abstract}
The multiplicity and roles of cytochrome P450 (CYP) genes in the 2 family are not well known in fish. Characterizations of CYP2 genes were done in the killifish, Fundulus heteroclitus. Multiple cDNAs from three CYP2 subfamilies were isolated from killifish cDNA libraries. Sequence analyses showed that one cloned from liver is related to trout CYP2K1. The other cDNAs were classified into two new subfamilies, CYP2N and CYP2P. CYP2N1, CYP2P2, and CYP2P3 were cloned from liver, and CYP2N2 was cloned from heart. CYP2P1 was cloned from a killifish genomic DNA library. Northern analyses showed that CYP2P transcripts are expressed strongly in gut and liver. Likewise, CYP2N1 transcripts are expressed strongly in liver and gut and also in heart and brain while CYP2N2 transcripts are expressed strongly in heart and brain and also in liver, gut, eye, kidney, and gill. The CYP2Ns and CYP2Ps are phylogenetically most closely related to the mammalian CYP2Js, and their transcripts also have similar tissue specific sites of expression as the CYP2Js. These phylogenetic and expression site similarities suggest functional similarities as well. To evaluate the functions of the killifish CYP, full length CYP2N1, CYP2N2, and CYP2P3 proteins were expressed in $S f 9$ insect cells using a baculovirus system, and their metabolism of arachidonic acid was examined. CYP2N1, CYP2N2, and CYP2P3 metabolized arachidonic acid at respective rates of 435, 95, and $135 \mathrm{pmol} / \mathrm{nmol} \mathrm{CYP} / \mathrm{min}$ forming a variety of eicosanoids including epoxyeicosatrienoic acids (14,15-, 11,12-, and 8,9-) and hydroxyeicosatrienoic acids (5-, 9-, 11-, 12-, 16-, 19-, and 20-). Eicosanoids, especially arachidonic acid metabolites, have been shown to modulate epithelial salt and water transport in a wide variety of organisms including mammals, frogs, toads, fishes, molluscs and insects. They also have been shown to act in various aspects of reproductive biology in sea stars, sea urchins, molluscs, insects, fishes, reptiles, and mammals. Arachidonic acid metabolites thus are involved in the regulation of cellular processes that are fundamental to organisms in general, and their synthesis is of primary interest. These results suggest that the CYP2Ns and CYP2Ps may be early vertebrate arachidonic acid catalysts.
\end{abstract}

Thesis supervisor: John J. Stegeman, Senior Scientist, WHOI 


\section{ACKNOWLEDGMENTS}

This work was dependent on the support and encouragement of many different people. First and foremost, I would like to thank John Stegeman who allowed me freedom to explore different aspects of P450 research. He was always ready with encouragement, good advice, and new ideas. Most importantly, he allowed me to make my own mistakes.

As well, I owe many thanks to the members of John's lab who daily provided help and input. These include Malin Celander, Celine Godard, Renee White, Eli Hestermann, Jennifer Schlezinger, Bruce Woodin, Rachel Cox, Michael Moore, Carolyn Miller, Michael Morss, and Shannon Bard. I especially thank Eli who has been a great office-mate with pertinent insight not only towards research questions, but also towards broad questions of philosophy, politics and life in general. I also thank Jennifer who was my fellow sufferer in North Carolina, Bruce who was always ready to help and who put a lot of effort into trying to purify proteins with me, Michael for his pithy comments on scientific life, Rachel for sequencing/phylogeny discussions, and Malin for her friendship and advice.

I also owe many thanks to the members of Mark Hahn's lab: Bonnie Woodward, Sibel Karchner, Brenda Jensen, Connie Hart, Diana Franks, and Sue Bello. Mark himself was ever helpful with sticky questions of evolution and phylogeny. Thanks to Sue, Fundulus were in constant supply. Sibel not only provided invaluable molecular biolgy advice (as well as that fantastic heart library!), she also was a great support and great friend. So too, was Connie Hart, my fellow graduate student and friend. Without Connie's laughter, friendship, and lessons in diplomacy and connecting senetences, graduate school would have been more formidable.

My thesis committee provided useful insights into this research, and I would like to thank Neal Cornell (MBL), Mark Hahn, Nancy Hopkins (MIT), and Judy McDowell. I also would like to thank Becky Gast who agreed to chair my thesis defense as probably her 
first official duty as a new scientist at Woods Hole.

This work would not have been possible without the Fundlus heteroclitus libraries provided by Doug Crawford (University of Missouri) and Sibel Karchner. As well, the arachidonic acid studies were only possible with the help of Darryl Zeldin and Shu Wu (NIEHS). The many members of his lab, especially Jim Boyle and Cindy Moomaw, made my stay in North Carolina more enjoyable. Cosette Sarbjit-Singh and Caroline Lee (Glaxo Pharmaceuticals) provided the vector containing the human reductase that was used in the expression studies. John Curtis (NIEHS) gave me unlimited use of his HPLC apparatus, and Carol Parker (U.S. EPA) injected and analyzed all of my gas chromatography/mass spectroscopy samples. Both Peter Smith and Kasia Hammer devoted time and effort trying to measure calcium flux in fish cells. In addition, I would like to thank Hank TrapedoRosenthal for letting me work in his lab at the BBS. Finally, Terry Rioux, Bob Adams, Bill Cruwys, Bob Wichterman, Judy Kleindinst, Lew Karchner, and everyone in the Education Office helped me in numerous ways.

All the members of Mitch Sogin's lab have helped me with phylogeny, especially Hilary Morrison, Greg Hinkle, and Jeff Silberman. Beck Gast also was always available to answer phylogenetic questions, even those somewhat unique to P450s.

I would not have completed this work without the encouragement and support of my family and friends. Thank you Mom and Dad, Sharon, Stephen, Babka, Sarah Huber and the whole Huber family, Janet Turley, Pratima Rao, Tiong Boon Ong, Karen Angelini and Cara Voutselas, Javier Escartin, Maureen Clayton, Gaspar Taroncher, Ee Lin Lim, Anya Waite, Emilie Hooft, Joanne Briana, my long suffering housemate Ken Gartner, Lew and Sibel, and Connie and Laird.

This work was funded by the Massachusetts Institute of Technology/ Woods Hole Oceanographic Institution Joint Program, U. S. EPA grant 823890, Seagrant NA46RG047O7/P-60, and the MIT fund for research at the Bermuda Biological Station. 
Abstract

Introduction

Material and Methods

Results

Discussion 
Chapter Three: Functional Characterisitics of Novel Cytochromes P450, CYP2N1 and CYP2N2, Highly Expressed in Killifish Heart and Brain $\quad 85$ $\begin{array}{ll}\text { Abstract } & 88\end{array}$

$\begin{array}{ll}\text { Introduction } & 89\end{array}$

$\begin{array}{ll}\text { Material and Methods } & 91\end{array}$

Results $\quad 94$

$\begin{array}{ll}\text { Discussion } & 107\end{array}$

Chapter Four: In vitro Expression of a Novel Cytochrome P450, CYP2P3: an Early Vertebrate Arachidonic Acid Catalyst 117

$\begin{array}{ll}\text { Abstract } & 118\end{array}$

$\begin{array}{ll}\text { Introduction } & 119\end{array}$

Material and Methods 121

$\begin{array}{ll}\text { Results } & 125\end{array}$

$\begin{array}{ll}\text { Discussion } & 133\end{array}$

$\begin{array}{lll}\text { Chapter Five: Summary and Conclusions } & 143\end{array}$

Cloning and Tissue Distribution 144

$\begin{array}{ll}\text { Phylogenetic Analyses } & 146\end{array}$

$\begin{array}{ll}\text { Functional Studies } & 146\end{array}$

$\begin{array}{ll}\text { Future Directions } & 154\end{array}$

Biological Effects 154

Regulatory Studies 155

$\begin{array}{ll}\text { Other substrates } & 157\end{array}$ 
Appendix A: Natural Products and P450s

Appendix B: Primary Hepatocytes 


\section{Chapter 1:}

Table 1: Subfamilies of hepatic cytochrome $\mathrm{P} 450$ proteins purified from fish

Chapter 2:

Table 1: CYP2P1 intron and exon sizes in kilobases $(\mathrm{kb})$

Table 2a: Percent nucleotide identities between killifish CYP2K2, CYP2N1, CYP2N2, CYP2P1, CYP2P2, and CYP2P3, trout CYP2K1, and rabbit CYP2J1

Table 2b: Percent amino acid identities between killifish CYP2K2, CYP2N1, CYP2N2, CYP2P1, CYP2P2, and CYP2P3, trout CYP2K1, and rabbit CYP2J1

\section{Chapter 3:}

Table 1: Regio- and stereochemical composition of EETs produced by recombinant CYP2N1

Table 2: Regiochemical composition of HETEs produced by recombinant CYP2N1

Table 3: Regio- and stereochemical composition of EETs produced by recombinant CYP2N2

Table 4: Regiochemical composition of HETEs produced by recombinant CYP2N2

Table 5: Regiochemical composition of DHETs produced by killifish microsomes 
Chapter 3:

Table 6: Regiochemical composition of HETEs produced by killifish microsomes

Table 7: Metabolic rates ( $\mathrm{pmol} / \mathrm{min} / \mathrm{nmol} \mathrm{CYP)} \mathrm{of} \mathrm{recombinant}$ CYP2N1, CYP2N2, and killifish liver microsomes incubated with different substrates

Table 8a: Comparison of the distribution and stereochemistry of the EETs and DHETs formed by recombinant CYP2N1, CYP2N2, and killifish liver microsomes

Table 8b: Comparison of the distribution of the HETEs formed by recombinant CYP2N1, CYP2N2, and killifish liver microsomes

Chapter 4:

Table 1: Regio- and stereochemical composition of EETs and 19-HETE produced by recombinant CYP2P3

Table 2: Metabolic rates ( $\mathrm{pmol} / \mathrm{min} / \mathrm{nmol}$ CYP) of recombinant CYP2P3 incubated with different substrates

Table 3: Comparison of the distribution and stereochemistry of the EETs produced by CYP2P3 to the same regioisomers produced by CYP2J2

Table 4: Comparison of the distribution and stereochemistry of the EETs and HETEs produced by CYP2P3 to the regioisomers produced by CYP2N1 and CYP2N2 


\section{Chapter 5:}

Table 1: Families and subfamilies of cytochromes $\mathrm{P} 450$ sequenced from fish 
LIST OF FIGURES

Chapter 1:

Figure 1: Fundulus heteroclitus (killifish)

Chapter 2:

Figure 1: Nucleotide and predicted amino acid sequence for killifish CYP2K2

Figure 2: Nucleotide and predicted amino acid sequence for killifish CYP2N1

Figure 3: Nucleotide and predicted amino acid sequence for killifish CYP2N2

Figure 4: Nucleotide and predicted amino acid sequence for killifish CYP2P2

Figure 5: Nucleotide and predicted amino acid sequence for killifish CYP2P3

Figure 6: Nucleotide and predicted amino acid sequence for killifish CYP2P1

Figure 7: Deduced amino acid alignment of killifish CYP2K2, CYP2N1 CYP2N2, CYP2P1, CYP2P2, and CYP2P3 with trout CYP2K1 52

Figure 8: Phylogenetic trees for CYP2 family proteins

Figure 9: Northern analyses of male and female livers probed with CYP2K2 CYP2N1, CYP2N2, CYP2P2, and CYP2P3 cDNAs

Figure 10: Northern analyses of male and female fore-gut, mid-gut and hind-gut probed with CYP2K2, CYP2N1, CYP2N2, CYP2P2, and CYP2P3 cDNAs 


\section{Chapter 2:}

Figure 11: Northern analyses of male and female extra-hepatic tissues probed with CYP2K2, CYP2N1, and CYP2N2 cDNAs

Figure 12: Northern analyses of extra-hepatic tissues of fed/starved/TPA reated fish probed with CYP2N1, CYP2N2, CYP2P2, and CYP2P3 cDNAs

Chapter 3:

Figure 1a: Spectrophotometric analysis of CYP2N1 expressed in $S f 9$ microsomes

Figure 1b: Spectrophotometric analysis of CYP2N2 expressed in $S f 9$ microsomes

Figure 2: Reverse-phase HPLC chromatogram of the organic soluble metabolites generated during incubation of recombinant CYP2N1 with $\left[1-{ }^{14} \mathrm{C}\right]$ arachidonic acid

Figure 3: Reverse-phase HPLC chromatogram of the organic soluble metabolites generated during incubation of recombinant CYP2N2 with $\left[1-{ }^{14} \mathrm{C}\right]$ arachidonic acid

Figure 4: Reverse-phase HPLC chromatogram of the organic soluble metabolites generated during incubation of killifish liver microsomes with $\left[1-{ }^{14} \mathrm{C}\right]$ arachidonic acid 
Chapter 4:

Figure 1: Spectrophotometric analysis of CYP2P3 expressed in Sf9 microsomes

Figure 2: Reverse-phase HPLC chromatogram of the organic soluble metabolites generated during incubation of recombinant CYP2P3 with $\left[1-{ }^{14} \mathrm{C}\right]$ arachidonic acid

Figure 3: Normal-phase HPLC chromatogram of the HETE metabolite generated during incubation of recombinant $\mathrm{CYP} 2 \mathrm{P} 3$ with $\left[1-{ }^{14} \mathrm{C}\right]$ arachidonic acid

Figure 4: Endogenous EETs recovered from liver and gut tissues

Figure 5: Endogenous DHETs recovered from liver and gut tissues

Chapter 5:

Figure 1: Arachidonic acid metabolism in vertebrates

Figure 2: Representative metabolites formed from arachidonic acid via cyclooxygenases, lipoxygenases, and cytochromes P450

Appendix A:

Figure 1: Total cytochrome P450 levels in Bermuda chub caught in 1988 (88-1-2), 1994 (94-1-5), and 1995 (95-1-15)

Figure 2: Immunoblots with Bermuda chub microsomes prepared from liver tissues 


\section{ABBREVIATIONS}

AEC: 3-amino-9-ethylcarbazole

AhR aromatic hydrocarbon receptor

ANOVA: analysis of the variance

1xBLOTTO: $\quad 5 \%$ nonfat dried milk, $0.02 \%$ sodium azide in $\mathrm{H}_{2} \mathrm{O}$.

Barbie barbiturate responsive element

BSA: $\quad$ bovine serum albumin

bp: $\quad$ base pair

cDNA: complementary DNA

cpm: $\quad$ counts per minute

CYP: $\quad$ cytochrome P450

CYPOR: $\quad$ NADPH cytochrome P450 reductase

dCTP: deoxycytidine triphosphate

50xDenhardt's: $1 \% \mathrm{w} / \mathrm{v}$ each Ficoll, polyvinylpyrrolidone, and BSA in $\mathrm{H}_{2} \mathrm{O}$

DEPC: diethyl pyrocarbonate

DHET: dihydroxyeicosatrienoic acid

DMSO: dimethyl sulfoxide

EET: $\quad$ epoxyeicosatrienoic acid

$\mathrm{EtBr}: \quad$ ethidium bromide

EtOH: ethanol

GC/MS: $\quad$ gas chromatography/mass spectroscopy

HETE: hydroxyeicosatrienoic acid

HPLC: $\quad$ high pressure liquid chromatography

IgG: $\quad$ immunoglobulin $\mathrm{G}$

ip: $\quad$ intraperitoneally 


\begin{tabular}{|c|c|}
\hline \multicolumn{2}{|c|}{ ABBREVIATIONS, continued } \\
\hline $\mathrm{J}:$ & joules \\
\hline kb: & kilobase \\
\hline $\mathrm{kDa}:$ & kilodalton \\
\hline MEM: & modified Eagle's medium \\
\hline MFO: & mixed function oxidase \\
\hline mRNA: & messenger RNA \\
\hline NADPH: & nicotinamide adenine dinucleotide phosphate \\
\hline PBS: & phosphate buffered saline \\
\hline PFA: & paraformaldehyde \\
\hline PFB: & pentafluorobenzyl bromide \\
\hline pfu: & plaque forming units \\
\hline PLHC: & Poeciliopsis lucida hepatoma carcinoma \\
\hline P/S: & penicillin/streptomycin \\
\hline SDS: & sodium dodecyl sulfate \\
\hline SEM & standard error of the mean \\
\hline Sf9 & Spodoptera frugiperda \\
\hline $20 \times S S C$ & $3 \mathrm{M} \mathrm{NaCl}, 0.3 \mathrm{M} \mathrm{NaCitrate}, \mathrm{pH} 7.0$ \\
\hline 20xSSPE & $3 \mathrm{M} \mathrm{NaCl}, 0.2 \mathrm{M} \mathrm{NaH}_{2} \mathrm{PO}_{4}, 20 \mathrm{mM}$ EDTA, pH 7.4 \\
\hline TCDD & $2,3,7,8$-tetrachlorodibenzo- $p$-dioxin \\
\hline TMS & bis(trimethylsilyl)trifluoracetamide \\
\hline TPA & 12-O-tetradecanoyl phorbol-13-acetate \\
\hline u & micro \\
\hline $\mathrm{uCi}$ & microCurie \\
\hline
\end{tabular}




\section{CHAPTER ONE}

INTRODUCTION 


\section{INTRODUCTION}

\section{Overall Objectives}

The long term goal of these studies is to determine the diversity, structure, function, and regulation of cytochromes $\mathrm{P} 450$ in early diverging vertebrates and to use this information to evaluate the probable evolution of the cytochromes $\mathrm{P} 450$ and their regulatory systems. To address this goal, we have begun to characterize cytochrome P450 2 (CYP2) genes and proteins in the marine teleost Fundulus heteroclitus.

\section{Why $\underline{P 450}$}

Cytochromes P450 are enzymes that transform the structure of organic chemicals via mixed-function oxidase (MFO) or monooxygenase reactions (Ortiz de Montellano, 1986). Cytochromes P450 constitute a gene superfamily (more than 400 sequenced to date), with members in bacteria, plants and animals (Nelson et al., 1996). Multiple forms of cytochrome P450 can be present and induced in a single organism. In vertebrates from fish to mammals the cytochrome P450 enzymes have been found throughout the body except in red blood cells, and they metabolize an extraordinary number and diversity of lipophilic compounds of both exogenous and endogenous origin. Cytochrome P450 activities with exogenously derived substrates might be considered primarily as adaptive functions, involving the protection against toxic chemicals. Cytochromes P450 metabolize many of these diverse compounds to products that are less bioactive and more readily excreted. However, metabolism can activate other compounds to derivatives that are more reactive and more toxic (Guengerich 1985). The broad tissue distribution of cytochromes P450 within a single organism and among many different organisms as well as their ability to oxidize compounds of widely different structure give cytochromes P450 a fundamental role in metabolic processes. 


\section{Why CYP2}

These studies concentrate on the CYP2 family for several reasons. First, roughly $20 \%$ of all CYP sequences are in family 2, and both the number and diversity of CYP2 subfamilies suggest that divergent ecological or physiological pressures may have affected this gene family and its expression. In addition, apparent differences in regulation occur among some CYP2s. Notably, CYP2B-like proteins in fish are not induced by the prototypic mammalian CYP2B inducers (Kleinow et al., 1987; Elskus and Stegeman, 1989; Elskus and Stegeman, 1989; Kleinow et al., 1990). This lack of induction has been speculated to reflect the lack of terrestrial plant material in the diets of the temperate fish most studied (Stegeman, 1993). Finally, CYP2 proteins in mammals metabolize a wide array of both endogenous and exogenous substrates. For example, CYP2A1 in rats is a testosterone $7 \alpha$-hydroxylase (Sonderfan et al., 1989), and human CYP2A6 is a coumarin hydroxylase (Yun et al., 1991). The CYP2Bs in several species metabolize testosterone and are chlorobiphenyl hydroxylases (Ryan et al., 1982). Human CYP2C8 and CYP2J2 are arachidonic acid epoxygenases (Rifkind et al., 1995; Wu et al., 1996). Many CYP2Cs and CYP2Js also metabolize benzphetamine (Bornheim et al., 1987; Zeldin et al., in press). CYP2D6 in humans activates opiates (Kerry et al., 1994). CYP2E1 in rats metabolizes ketones, ethanol, benzene, and trichloroethylene (Nanji et al., 1994). Given the diversity and number of mammalian CYP2 proteins, one might ask whether CYP2 genes in other vertebrate groups are as diverse and numerous as they are in mammals.

\section{Why Fish}

Fish constitute the largest and earliest diverging vertebrate family $(\sim 30,000$ fish species vs. $\sim 4,000$ mammalian species). They first evolved $500+$ million years ago, roughly 300 million years before mammals (Nelson, 1984). Knowledge of CYP in primitive vertebrates has important implications for understanding the evolution of vertebrate 
CYPs in general. Sequence analysis of CYP in different vertebrate groups is an important way to address the evolution of this gene superfamily in vertebrates. Highly conserved CYP between fish and mammals would suggest conserved and possibly endogenous functions. Differences in CYP between fish and mammals could underlie fundamental organismal differences.

Studies to date have shown that fish have multiple CYP2 proteins. In several fish species, immunoblot analyses have shown CYP2B-like and CYP2E-like proteins (Gray et al., 1991; Stegeman and Hahn, 1994). The lauric acid hydroxylases, CYP2K1 and CYP2M1 have been isolated from trout, and again, immunoblot analyses have shown CYP2K-like and CYP2M-like proteins in different fish species (Miranda et al., 1989; Schlenk et al., 1993). As well as having unique CYP proteins, fish probably have either orthologues or paralogues of many mammalian CYP2 proteins.

The myriad proteins in the CYP2 family are postulated to have arisen in order to metabolize dietary plant natural products (Nelson and Strobel, 1987). Unlike mammals, the majority of fish are carnivorous and encounter fewer plant natural products (Horn, 1989). As discussed above, total CYP levels are not induced by the prototypic CYP2 inducer, phenobarbital, in the temperate carnivorous fish most studied, and this lack of induction has been postulated to reflect the lack of terrestrial plants in their diets (Stegeman, 1993). If CYP2 proteins first evolved to metabolize endogenous substrates and later expanded and diversified in order to cope with dietary plant natural products, then fish that have not had to cope with these natural products may have less diverse CYP2 proteins. Thus, fish may be a good vertebrate system with which to examine interactions between CYP and endogenous substrates, and knowledge of fish CYP2 proteins might have important implications for identifying endogenous CYP2 substrates.

This research focuses on fish P450 proteins in the 2 family. Fundamental questions remain concerning their identity and number, their catalytic functions, their 
regulation and expression at the genetic as well as the organismal level, their evolution and inter-relatedness, and finally, their endogenous functions, if any. Cytochrome P450 proteins have been studied mainly in mammals. Fish have penetrated virtually every conceivable water habitat and range from the ocean depths $(-11,000 \mathrm{~m})$ to high mountain streams $(+4,500 \mathrm{~m})$ and from hot springs $\left(43^{\circ} \mathrm{C}\right)$ to subfreezing water $\left(-1.8^{\circ} \mathrm{C}\right)$ (Lauder and Liem, 1983). Because fish inhabit diverse and extreme environments throughout the world's waters, they can be expected to have novel P450 proteins not found in mammals as well as forms similar to those in mammals.

\section{CYTOCHROME P450 BACKGROUND}

\section{General Reaction}

Oxidative metabolism involving molecular oxygen is the initial enzymatic process in the biotransformation of a majority of lipophilic organic compounds. The general scheme for monooxygenase reactions is:

$$
\mathrm{RH}+\mathrm{NADPH}+\mathrm{O}_{2}+\mathrm{H}^{+}------>\mathrm{ROH}+\mathrm{NADP}^{+}+\mathrm{H}_{2} \mathrm{O}
$$

where RH is the substrate and ROH is the hydroxylated product (Stegeman and Hahn, 1994). Cytochrome P450 proteins catalyze oxygenase reactions: they catalyze the incorporation of a single atom of molecular oxygen into its substrate while the other oxygen is reduced to water. They belong to a large and expanding superfamily of heme protein monooxygenases (Nelson et al., 1996). Some P450 proteins have multiple substrates, regulatory mechanisms, and/or biological functions. Altogether, the cytochrome P450 proteins catalyze thousands of different reactions including both oxidative and reductive reactions as well as the release of superoxide anions (Ortiz de Montellano, 1986). 


\section{$\underline{\text { P450 Properties }}$}

Most P450 enzymes are discrete gene products of about 57,000 kDa (about 500 amino acid residues) (Soucek and Gut, 1992) and contain one equivalent b-type heme per polypeptide (Black and Coon, 1987). Microsomal P450s contain a highly hydrophobic signal/anchor segment at the amino terminus followed by a short, cationic, halt transfer sequence. In contrast, mitochondrial P450s have a transient targeting sequence at the amino terminus which is proteolytically removed after transport of the precursor to the mitochondrial inner membrane. The mature proteins lack hydrophobic amino termini but otherwise are similar to microsomal P450s. The amino terminus is the principle site of membrane binding, and the majority of the polypeptide is exposed to the cytosolic (inner mitochondria) side of the membrane (Korzekwa and Jones, 1993). All cytochrome P450 proteins possess a noncovalently bound heme (protoporphyrin IX) and a segment of twenty-six amino acids surrounding a cysteine that is highly conserved. This cysteine donates the thiolate fifth ligand to the heme iron (Ortiz de Montellano, 1986).

The amino acid environment surrounding the heme results in a typical cytochrome P450 Soret absorption band at about $450 \mathrm{~nm}$ when the iron is reduced by electrons and complexed with carbon monoxide (Ortiz de Montellano, 1986). This property of the cytochrome pigment absorbing at $450 \mathrm{~nm}$ gives the enzyme its common name, cytochrome P450. Specific cytochrome P450s are named with the root CYP derived from cytochrome P450 followed by an Arabic numeral denoting the gene family, a letter for the subfamily, and another Arabic numeral designating the gene number (Nelson et al., 1993). P450s within a single gene family generally exhibit more than $40 \%$ sequence similarity. P450s within the same subfamilies generally have more than $55 \%$ identity within the same

species. Cytochromes P450 with the same gene number in different species are considered orthologous. 


\section{Evolution of $\underline{\mathrm{P} 450}$ Proteins}

Cytochrome P450 proteins are ancient. Estimates based on P450 sequences suggest that P450 genes originated between 1.5 and 3 billion years ago (Stegeman and Hahn, 1994). P450 proteins also are relatively simple. Some P450 catalyzed reactions can be mimicked by heme analogs in an organic solvent: in these model systems, the protein part of the P450 is not essential to the reaction (Mansuy et al., 1989). Thus, the protein part of the enzyme does not seem to have a direct catalytic function. It appears to be largely limited to providing to the heme an environment conducive to the reactions catalyzed and to controlling the access of substrates to the heme. Consequently, even minor alterations of the protein can lead to changes of enzyme specificity: substitutions of a single amino acid residue have been shown to alter substrate recognition patterns (Lindberg and Negishi, 1989).

With the proper modifications, such as increased flexibility or a larger binding site, a P450 protein with very broad specificity probably could have evolved. But instead of only a few P450 proteins with very broad specificities, a vast number of P450 proteins with somewhat limited, but overlapping, specificities evolved, perhaps in order to avoid interference with metabolism of endogenous substrates (Nebert, 1991). Thus, cytochrome P450 proteins capable of metabolizing a diversity of exogenous compounds may have been constrained by the need not to metabolize endogenous compounds which overruled the advantage inherent in making only a few proteins to metabolize many different exogenous compounds (Zimniak and Waxman, 1993). The functional adaptability of P450 proteins may have profound evolutionary consequences. Changes of one or a few amino acids are relatively frequent and could lead to the emergence of cytochrome P450s capable of metabolizing a phenomenal number of compounds such as toxic plant products to which animals may be exposed after colonization of dry land and man-made synthetic compounds which previously were absent from the environment. 
In general, membrane bound $\mathrm{P} 450$ proteins with a flavoprotein as their immediate redox partner (eukaryotic P450s) are more closely related to each other than to soluble P450 proteins with an iron-sulfur protein as their immediate redox partner (prokaryotic P450s) and vice versa. CYP102, a soluble, prokaryotic enzyme that clusters with the eukaryotic P450s in phlogenetic analyses is the only known exception (Gonzalez and Nebert, 1990). Certain bacterial and eukaryotic cytochromes $\mathrm{P} 450$ receive electrons via an iron-sulfur protein which itself receives electrons from a flavin adenine dinucleotide containing enzyme. In eukaryotes, this first major class of P450s is found exclusively in the inner mitochondrial membrane, is involved in highly specific steroid biosynthesis pathways, and does not metabolize xenobiotics. Endogenous P450 functions include sterol metabolism, fatty acid oxidations and oxidations of ethanol and other low molecular weight compounds, and the biosynthesis and metabolism of cholesterol to bile acids and steroid hormones. In contrast to many xenobiotics, endogenous compounds often are hydroxylated by individual P450s with a high degree of positional and stereochemical selectivity (Waxman et al., 1991).

The second major class of P450s is bound to microsomal membranes and electrons are donated to these enzymes via a protein containing a single molecule each of flavin adenine dinucleotide and flavin mononucleotide. A few microsomal P450s are involved in steroidogenesis in specialized tissues. Others are involved in the cholesterol-bile acid biosynthetic pathway (Gonzalez, 1989). However, the majority of microsomal P450s metabolize foreign compounds. In mammals, cytochromes P450 in gene families 1-4 are the most prominent in the metabolism of xenobiotics. Many genes in these families also are induced by xenobiotics. P450s in families 1-4 catalyze many activities with much overlap, and few substrates are exclusive to one protein. As well, orthologous P450s in different species may have different substrate specificities. 


\section{CYP Families 1-4}

The majority of information on cytochromes P450 in animals comes from studies in mammalian species. Experiments with rats, mice, rabbits and humans provide most of the fundamental knowledge concerning the enzymes in CYP gene families 1 through 4 .

CYP 1: The CYP1 family has an affinity for planar substrates and is able to oxygenate molecules in conformationally hindered (bay region) positions, resulting in their activation (Parke et al., 1991). In mammals, the CYP1A subfamily comprises two enzyme proteins, CYP1A1 and CYP1A2. CYP1A1 reacts with aromatic hydrocarbon substrates such as benzo[a]pyrene and 3-methylcholanthrene. CYP1A2 reacts with aromatic amines and amides such as B-naphthylamine and 2-acetylaminofluorene (Parke et al., 1991). Regulation of cytochrome P450 activity may occur at the transcriptional, translational, and/or post-translational level. The regulation of CYP1A is the most well characterized of P450 regulation at the molecular level. Briefly, upon treatment with an inducer, an Ah receptor (aromatic hydrocarbon receptor) forms a complex with the hydrophobic ligand inducer and is translocated into the nucleus. The complex reacts with 5 regulatory regions of the CYP1A gene and induces transcription of the CYP1A genes among others (Hankinson, 1995). CYP1B recently has been cloned in mammals and also metabolizes aromatic hydrocarbons (Savas et al., 1994; Sutter et al., 1994). The CYP1B proteins are expressed constitutively at low levels in heart, brain, placenta, lung, skeletal muscle, kidney, spleen, thymus, prostate, gonads, small intestine, colon, and peripheral blood leukocytes (Savas et al., 1994; Sutter et al., 1994).

CYP 2: P450s in gene family 2 are numerous and diverse. Because the mammalian CYP2 family markedly expanded within the past 400-800 million years, about the same time that land plants first evolved, it has been hypothesized that the CYP2 family 
diversified to metabolize plant secondary metabolites (Nelson and Strobel, 1987). This large diversity in the CYP2 family may mask orthologous relationships between the CYP2 genes. The following sections briefly summarize some of the information concerning the CYP2 subfamilies.

CYP2A: The rat CYP2A1 and CYP2A2 are differentially regulated and have distinct substrate specificities: CYP2A1 is involved in the $7 \alpha$-hydroxylation of testosterone in young male and female rats while CYP2A2 is only expressed in adult male rats and has a high level of $15 \alpha$-hydroxylase activity towards progesterone (Aoyama et al., 1990). CYP2A3 is expressed only in rat lung and is induced by 3-methylcholanthrene via transcriptional activation (Gonzalez, 1989). The sex-specific expression of the rat CYP2As is controlled in part by secretion of pituitary hormone (Waxman et al., 1985; Waxman et al., 1989).

CYP2B: The CYP2B subfamily contains at least ten members. Mammalian CYP2B proteins function in the metabolism of a wide array of drugs, natural products, and pollutants. The proteins are induced by many of their substrates, typified by phenobarbital but including polychlorinated biphenyl congeners, chlorinated pesticides, and many tumor promoters. CYP2B prefers globular molecules for substrates and generally initiates their detoxification. In rat liver, CYP2B1 and CYP2B2 are the major phenobarbital-inducible P450 enzymes. These enzymes have $97 \%$ sequence similarity, but CYP2B2 is generally of lower catalytic activity. CYP2B1 actively metabolizes a broad spectrum of lipophilic drugs and steroidal substrates including androgens such as androstenedione. CYP2B2 has a similar but distinguishable substrate specificity profile but is several-fold less active than CYP2B1 with many monooxygenase substrates (Gonzalez, 1989). The induction of CYP2B activity by phenobarbital is primarily due to new CYP2B protein synthesis that 
results from an increase in steady state levels of CYP2B mRNA resulting from increased transcription of the corresponding CYP2B genes. This transcriptional activation is rapid (30-60 minutes) and can reach a level 20-50 times higher than the basal transcription rate (Adesnik et al., 1981).

CYP2C: The CYP2Cs generally are expressed constitutively and are under developmental and sex-specific regulation (Gonzalez, 1989). CYP2Cs metabolize steroids such as testosterone and progesterone. In humans, CYP2C9 is important in the metabolism of pharmaceuticals. Pharmaceutical substrates include S-warfarin, tolbutamide, phenytoin, tertrahydrocannabinol, ibuprofen, naproxen, tienilic acid, and dicifenac (Smith and Jones, 1992).

CYP2D: The CYP2Ds are among the most important CYP involved in the metabolism of drugs by humans (Smith and Jones, 1992). The CYP2Ds catalyze a suite of reactions including aromatic hydroxylation (propanolol), aliphatic hydroxylation (metoprolol), $\mathrm{N}$-dealkylation (amiflamine), and hydroxyl oxidation (reduced haloperidol). Other CYP2D substrates include oxyprenolol, codeine, debrisoquine, imippramine, and thioridazine, to name a few (Smith and Jones, 1992). Substrates for CYP2D6 have a basic atom about 5-7 $\AA$ from the site of metabolism which suggests that the enzyme has an acidic residue at approximately this distance from the iron atom (Smith and Jones, 1992).

CYP2E: CYP2E metabolizes small molecules including ethanol, acetone, benzene, and dialkyl nitrosamines and probably is involved in cholic acid biosynthesis (Ryan et al., 1985). CYP2E tends to generate reactive oxygen radicals and is induced by starvation, ethanol, and acetone (Parke et al., 1991). Induction mechanisms include transcriptional activation, mRNA stabilization, and protein stabilization (Soucek and Gut, 1992). 
CYP2F: CYP2F was cloned from a human lung CDNA library and has been shown to activate the pneumotoxin naphthylamine (Nhamburo et al., 1989). The endogenous functions of CYP2F proteins are unknown.

CYP2G: Rat CYP2G is a neuroepithelial enzyme and is thought to be involved in olfactory sensing (Nef et al., 1989; Nef et al., 1990). Rabbit CYP2G1 was isolated from a rabbit nasal cDNA library (Ding et al., 1991), is only detected in the olfactory mucosa (Ding and Coon, 1990), and is a principal sex steroid hydroxylase in olfactory microsomes (Ding et al., 1991).

CYP2H: CYP2H1 and CYP2H2 were isolated from chick hepatocytes (Hobbs et al., 1986; Hansen and May, 1989). Reconstituted CYP2H1 and CYP2H2 both demethylate benzphetamine equally but differ in their ability to metabolize p-nitrophenol and acetominophen (Sinclair et al., 1990). In chick hepatocytes, in vivo and in vitro, phenobarbital treatment results in a fifty fold induction of CYP2H1 and CYP2H2 mRNAs. These mRNAs share $92 \%$ deduced amino acid similarity with each other and share 51-56\% amino acid sequence similarity with several phenobarbital-inducible and constitutive mammalian CYP2C forms (Hobbs et al., 1986; Hansen and May, 1989).

CYP2J: CYP2Js have been identified in humans, rats, mice and rabbits (Wu et al., 1996; Wu et al., 1997; Zhang et al., 1997). Most are highly expressed in gut and liver, and some are expressed in a variety of other tissues including heart. It is not yet known whether the CYP2Js are inducible. Recently, some CYP2J proteins have been shown to metabolize arachidonic acid with a high degree of regio- and stereospecificity (Wu et al., 1996; Wu et al., 1997; Zhang et al., 1997). 
CYP2L: The CYP2Ls from lobster are the only full-length, invertebrate CYP2s yet identified (James et al., 1993). The CYP2Ls are highly expressed in the hepatopancreas (digestive organ) and catalyze the monooxygenation of benzphetamine, aminopyrine, benzo[a]pyrene, progesterone, and testosterone. CYP2L1 sequence recently has been published (James et al., 1996), and another CYP2L sequence, CYP2L2, has been identified (Boyle and James, unpublished data).

CYP2K and CYP2M: The trout CYP2K1 and CYP2M1 proteins both are lauric acid hydroxylases (Buhler et al., 1994; Yang et al., 1995) and are discussed further in the section on cytochromes P450 in fish.

CYP2Q: CYP2Q recently was sequenced from frog (Ohi et al., unpublished data). No data on properties other than sequence is available for CYP2Q.

Altogether, the CYP2 family proteins metabolize a wide variety of different compounds. As discussed above, the proliferation and diversification of the CYP2 family has been suggested to have arisen in order to metabolize dietary, plant natural products. Similarly, the large number of substrates including natural products that CYP3 proteins metabolize also suggests involvement of the CYP3 gene family with natural products.

CYP3: The CYP3 family also has a large number of genes in different species, but unlike the CYP2s, the CYP3s all belong to the same subfamily, CYP3A. The CYP3As have an affinity for large, nonplanar substrates such as the macrolide antibiotics and are known to metabolize cyclosporine, ergotamine derivatives, and some steroids. CYP3A proteins are induced by pregnenolone $16-\alpha$-carbonitrile. CYP3A is the principal P450 of human liver and is involved in the activation of the dihydrodiol derivatives of polycyclic 
aromatic hydrocarbons to the ultimate mutagens and carcinogens (Parke et al., 1991).

CYP4. Enzymes in this family oxidatively metabolize carboxylic acids such as arachidonic acid at the $\omega$ or $\omega-1$ carbon groups and thus may be involved in pathways of fatty-acid and prostaglandin oxidation. This family is induced by the antihypercholesteraemic drugs, clofibrate and ciprofibrate, and the phthalate and sebacate ester plasticizers and is associated with peroxisome proliferation (Parke et al., 1991).

\section{Cytochromes P450 in Fish}

Catalytic, immunochemical and regulatory studies indicate that CYP families 1-4 also occur in fish (Stegeman, 1989; Stegeman and Hahn, 1994). Evidence summarized in Table 1 is drawn from recent reviews (Stegeman, 1987; Stegeman, 1993).

Table 1. Subfamilies of Hepatic Cytochrome P450 Proteins Purified from Fish

\begin{tabular}{|c|c|c|}
\hline$\underline{\text { Subfamily }}$ & Trivial Name & Evidence \\
\hline $1 \mathrm{~A}$ & $\begin{array}{l}\text { Scup P450 E } \\
\text { Trout LM4b } \\
\text { Cod c } \\
\text { Perch V }\end{array}$ & $\begin{array}{l}\text { P-C, F, I, R, S } \\
\text { P-C, F, I, R, S } \\
\text { P-C, F, I, R, } \\
\text { P-C, F, I, R }\end{array}$ \\
\hline $2 B$ & Scup P450 B & I, S \\
\hline $\begin{array}{l}2 \mathrm{~K} \\
2 \mathrm{M}\end{array}$ & $\begin{array}{l}\text { Trout LM } 2 \\
\text { Trout LMC } 1\end{array}$ & $\begin{array}{l}\text { I, S } \\
\text { I, S }\end{array}$ \\
\hline & Trout KM2 & I \\
\hline $\begin{array}{l}3 \mathrm{~A} \\
3 \mathrm{~A} 27\end{array}$ & $\begin{array}{l}\text { Scup P450 A } \\
\text { Trout LMC } 5 \\
\text { Trout P450 con } \\
\text { Cod b }\end{array}$ & $\begin{array}{l}\text { F, I } \\
\text { F, I, S } \\
\text { F, I } \\
\text { I }\end{array}$ \\
\hline
\end{tabular}

Subfamilies $2 \mathrm{E}, 2 \mathrm{C}$ and $4 \mathrm{~A}$ have been indicated in one or more species by presence of cross-reactive proteins in microsomes.

Assignment to subfamilies is based on: $\mathrm{P}-\mathrm{C}=$ physico-chemical properties; $\mathrm{F}=$ functional properties; $\mathrm{I}=$ immunological cross reactivity; $\mathrm{R}=$ regulatory similarities; $\mathrm{S}=$ sequence similarities. 
Sequences for CYP1A genes have been identified in a variety of other teleosts. These include plaice (Leaver et al., 1993), oyster toadfish (Morrison et al., 1995), four-eye butterfly fish (Vrolijk et al., 1995), european sea bass (Stien et al., 1995), and wild red sea bream (Mizukami et al., 1994). In additon, a partial CYP3A cDNA, CYP3A30, has been sequenced in killifish (Celander and Stegeman, 1997) and a partial CYP4 cDNA, CYP4T1, has been sequenced in rainbow trout (Falckh et al., 1997).

\section{Teleost CYP1A}

A close immunochemical relationship exists between CYP1A in fish and mammals and among vertebrates generally. This relationship also is based on deduced amino acid sequence similarities. In fish, a single dose of $\beta$-naphthoflavone causes an increase in CYP1A mRNA by 6 hours with a maximum level reached at 30-40 hours. This declines to near control levels at 4-5 days. Functional protein is made by 18 hours with a maximum at three days. Elevated protein levels can persist for 2-3 weeks (in mammals, persistence is generally less than 48 hours) (Kloepper-Sams and Stegeman, 1989). A less rapidly metabolized inducer can cause high mRNA levels for up to two weeks (Hahn and Stegeman, 1994). High dose and/or prolonged exposure causes a decline in activity and in protein even while mRNA levels are maintained (White et al., 1997).

\section{Teleost CYP2B}

A number of fish have liver microsomal proteins that cross-react with antibodies to various mammalian CYP2 family proteins. One purified P450 from fish has sequence showing a relationship to the phenobarbital-inducible CYP2B: the amino terminal sequence comparisons show that $\mathrm{P} 450 \mathrm{~B}$ from scup has $50+\%$ sequence identity to mammalian CYP2Bs, suggesting identity as a $2 \mathrm{~B}$ family member. 


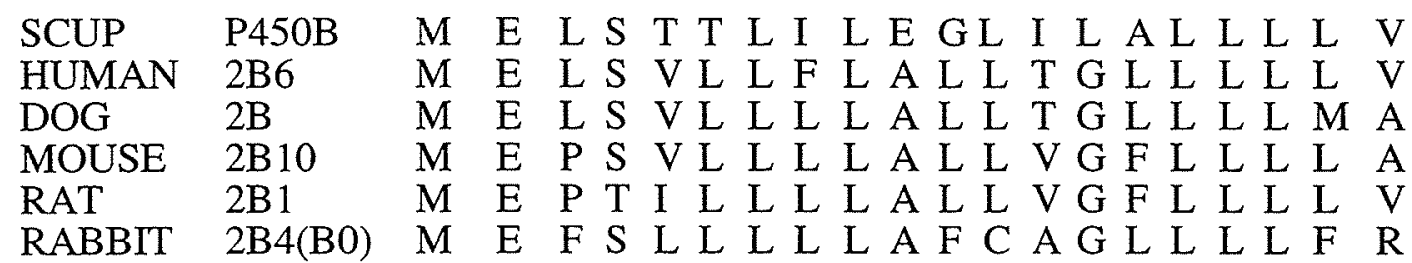

Anti-P450B recognizes two proteins in scup liver microsomes, one major and one minor. Scup P450B is an immunological counterpart to the PB-inducible $2 \mathrm{~B} 1$ forms in mammals based on reciprocal analysis of purified scup P450B and rat P4502B1 and antibodies to these antigens. Anti-scup $\mathrm{B}$ recognizes pure rat $2 \mathrm{~B} 1,2 \mathrm{~B} 2$, and $2 \mathrm{~B} 3$ and only those proteins in rat liver microsomes. Anti-rat $2 \mathrm{~B} 1$ recognizes scupB and the same size protein in scup liver microsomes (Stegeman et al., 1990). Specific protein bands in Western blots of all fish, birds, and mammals (except whales) examined are detected identically with antibodies to either rat P450 2B l or to scup P450B. In addition, a cDNA probe for rat $\mathrm{P} 4502 \mathrm{~B} 1 / 2$ detected a hybridizing RNA sequence in scup liver.

Despite the evidence for a teleost 2B-type protein, fish have not been found to respond in any recognizable way to typical mammalian CYP2B inducers such as phenobarbital (Kleinow et al., 1987; Elskus and Stegeman, 1989; Elskus and Stegeman, 1989; Kleinow et al., 1990). Interestingly, some other non-mammalian vertebrates including reptiles and amphibians, also lack 2B-like induction (Stegeman and Hahn, 1994). An examination of the fish CYP that are homologous to the mammalian phenobarbital inducible genes, but are not phenobarbital inducible, may provide insight into the molecular mechanisms that govern the selectivity of phenobarbital induction for individual P450 genes.

\section{Teleost CYP2E}

Evidence for teleost CYP2E proteins comes from functional, immunochemical, and 
mRNA studies. Fish liver microsomes were shown to dealkylate diethylnitrosamine (Kaplan et al., 1991), an activity associated with CYP2E enzymes in mammals (Gonzalez, 1989). Antibodies to rat CYP2E1 recognized a single protein in the liver of Poeciliopsis sp. Finally, an oligonucleotide probe specific for rat CYP2E1 hybridized with a $3.3 \mathrm{~kb}$ mRNA in RNA samples from Poeciliopsis sp. This mRNA also was induced by ethanol (Kaplan et al., 1991), a prototypic CYP2E inducer.

\section{Teleost CYP2K}

Trout LM 2 was classified into the novel CYP2K subfamily based on its sequence (Buhler et al., 1994). CYP2K1 shows marked differences in sex and tissue-specific expression at both the transcriptional and translational level in sexually mature, rainbow trout liver and trunk kidney. CYP2K1 cDNA hybridized with a 2.8 and a $1.9 \mathrm{~kb}$ mRNA in mature male trunk kidney and some male livers. In females, the $2.8 \mathrm{~kb}$ band generally was not detected. CYP2K1 transcript was expressed more strongly in kidney than in liver and also was expressed more strongly in males than females. These differences showed corresponding differences in protein expression and activities (Buhler et al., 1994). Constitutive CYP2K1 exhibits high estradiol 2-hydroxylase activity and also catalyses the $\omega-1$ hydroxylation of lauric acid and the conversion of aflatoxin B1 to the highly electrophilic aflatoxin B1-8,9-epoxide metabolite (Williams et al., 1984). CYP2K3 also has been identified in trout and shares $96.5 \%$ deduced amino acid identity with CYP2K1 (Buhler, unpublished data).

\section{Teleost CYP2M}

Trout LMC1 recently was classified into the novel CYP2M subfamily based on its sequence (Yang et al., 1996). The predicted amino acid sequence of CYP2M1 is 57\% identical to that of CYP2K1. Northern analyses of trout RNA showed that CYP2M1 
hybridized to a single $2.2 \mathrm{~kb}$ RNA in liver but not kidney. Like CYP2K1, CYP2M1 also metabolizes lauric acid; CYP2M1 shows $\omega-6$ hydroxylase activity towards lauric acid.

\section{Teleost CYP3A}

Trout P450con, trout LMC5, scup P450A, and cod P450b are immunochemically related; in addition, each was recognized by polyclonal antibodies to rat CYP3A1 and human CYP3A4 suggesting that these fish proteins all are CYP3A-like and probably members of the CYP3A subfamily (Celander et al., 1996). Furthermore, trout LMC5 and scup P450A are the major hepatic microsomal enzymes that metabolize testosterone at the $6 \beta$-position; this activity is associated with CYP3A enzymes in mammals (Waxman, 1988). Sequence analysis of trout LMC5 has confirmed this protein as a CYP3A family member (Buhler, unpublished data) and partial sequence for a killifish CYP3A demonstrates this gene in killifish (Celander and Stegeman, 1997).

The fish CYP3A-like proteins are present at high levels in untreated fish (Celander et al., 1989; Gray et al., 1991). In contrast to mammals, little is known about the inducibility of the CYP3As in fish. They seem to be slightly induced by steroids (Celander et al., 1989) and may be influenced by dietary natural products (Vrolijk et al., 1994). Finally, sex-differences may depend on species differences in fish CYP3A-like protein levels and activities (Celander et al., 1996). The mechanism of the regulation of the fish CYP3 genes is still unknown.

\section{Teleost CYP4T}

A partial CYP4T1 sequence recently was cloned from trout liver (Falckh et al., 1997). CYP4T1 deduced amino acid sequence is most similar to rat CYP4B2 with $55.4 \%$ identity. 
In addition to CYP in families 1-4, CYP in other families have been identified in fish. The cholesterol side-chain cleavage cytochrome P450, CYP11A1, has been cloned from both rainbow trout (Takahashi et al., 1993) and southern stingray (Nunez and Trnat, 1996). The 17 alpha-hydroxylase, CYP17, has been cloned from rainbow trout (Sakai et al., 1992), medaka (Kobayashi et al., 1996), and dogfish (Trant, 1995). Aromatase or CYP19 has been cloned from channel catfish (Trant, 1994), rainbow trout (Tanaka et al., 1992), goldfish (Gelinas et al., 1996), medaka (Tanaka et al., 1995), and tilapia (Chang et al., 1996). Recently, a CYP in a new vertebrate family, CYP26, was isloated from zebrafish (White et al., 1996). CYP26 is a retinoic acid-inducible all trans retinoic acid 4hydroxylase. Finally, a partial sequence for a CYP52 has been cloned from rainbow trout (Gong, 1994).

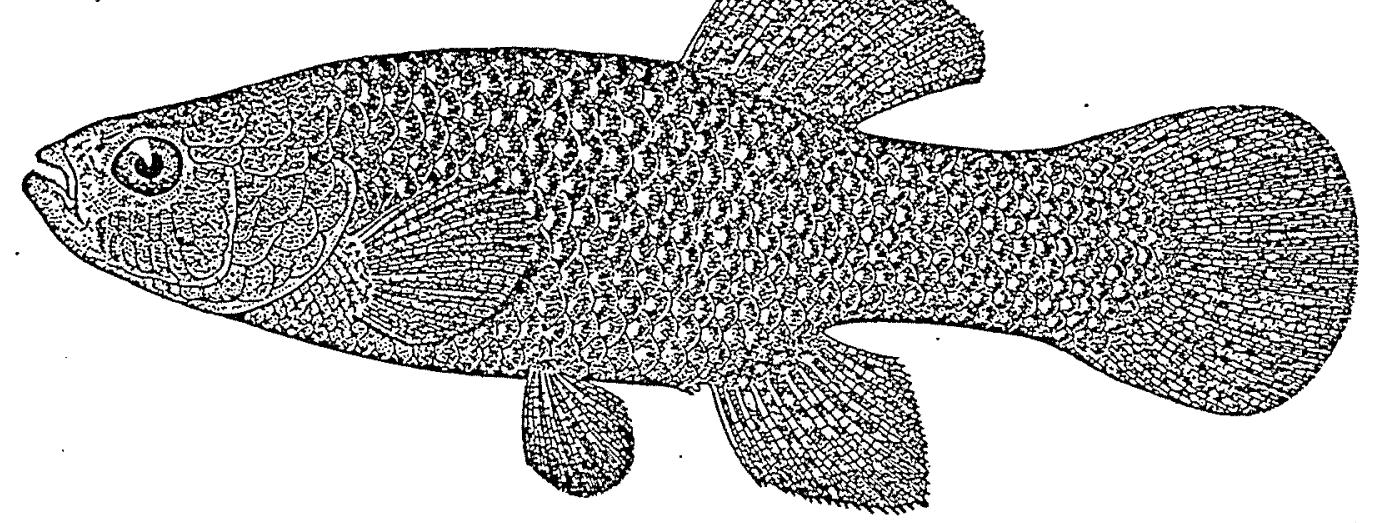

actual size:

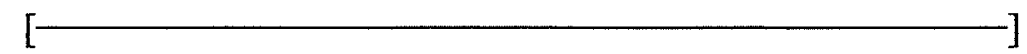

Figure 1. Fundulus heteroclitus (killifish). From (Bigelow and Schroeder, 1953).

Specific Objective and Approach

Although the foregoing indicates that members of gene families 1-4 are expressed in fishes, the studies in fishes have not approached the question of gene diversity in the largest mammalian CYP family, family 2. The specific objective of this research is to explore the diversity, structure, function, and regulation of fish cytochromes P450 in family 2 and to use this information to evaluate the probable evolution of the cytochromes 
P450 and their regulatory systems. We approached this objective in the marine teleost, Fundulus heteroclitus or killifish. We used killifish for several reasons.

1. Killifish are an abundant and common fish on the east coast of North America. They are widespread and occur in virtually every salt marsh from Canada to Virginia. We have many different populations in Woods Hole, and they are obtained easily.

2. Killifish reside in salt marshes and range only a few hundred yards from their natal creeks (Lotrich, 1975) making them good species for environmental toxicology studies.

3. Killifish are extremely hardy and adapt well to almost any laboratory condition. They can be cultured easily from eggs and sperm in the laboratory.

4. Killifish move onto marshes with the incoming tide to feed and are an important link in the energy transfer between the shore and open waters (Penczak, 1985). In the winter, killifish hibernate unfeeding in mud (Valiela et al., 1977). They are mainly carnivorous although algal filaments and detritus are common gut contents (D'Avanzo and Valiela, 1990). Because of their significant detrital dietary component and their coastal habitat, they likely are exposed to a wide variety of pollutants from both the water and the sediments.

5. Extensive literature exists on the population genetics of this species in the Eastern United States (Powers et al., 1986; Bernardi et al., 1993).

6. The reproductive physiology of killifish has been studied in detail (Selman and Wallace, 1986; Hsiao and Meier, 1989; Hsiao et al., 1994).

7. The embryology and developmental biology of killifish is known better than that of any other marine or estuarine fish (Armstrong and Child, 1965).

8. Analysis of liver microsomes show that killifish express multiple CYP2-like proteins (Gray et al., 1991).

9. Both genomic and cDNA libraries from liver and heart of killifish were 
available. Dr. Douglas Crawford at the University of Chicago provided a genomic DNA library and a liver cDNA library. Dr. Sibel Karchner at Woods Hole Oceanographic Institution provided a liver cDNA library and a heart cDNA library.

The approach taken for this work was to design degenerate oligonucleotide probes to conserved sequences in multiple CYP2 genes. These probes were used to screen the killifish libraries. Initially, partial sequences in three CYP2 subfamilies, CYP2K, CYP2N, and CYP2P were isolated. These partial sequences were used to further screen the libraries and obtain full length sequences. Chapter 2 details these initial experiments and describes the phylogenetic relationships between the killifish CYP2 genes and other CYP2 genes. The next question addressed concerned the functions of these genes. To approach this question, three of these genes, CYP2N1, CYP2N2, and CYP2P3, were co-expressed with human NADPH cytochrome P450 reductase (oxidoreductase) in Sf9 insect cells using a baculovirus expression system. Functional P450 and reductase proteins were co-expressed in the insect cells and used in catalytic studies. The results of these experiments are presented in Chapters 3 and 4. Finally, Chapter 5 briefly summarizes the results of these studies and discusses future research directions. 


\section{CHAPTER TWO}

DIVERSITY OF NOVEL CYP2 GENES IN FUNDULUS HETEROCLITUS 


\section{ABSTRACT}

The cytochrome P450 2 (CYP2) genes occupy 15 subfamilies in mammals (Nelson et al., 1996). The diversity of non-mammalian vertebrate genes in the CYP2 family is not known but could shed light on the functional significance of CYP2 diversity. Genes in the CYP2 family were sought in the killifish (Fundulus heteroclitus). Multiple cDNAs related to known CYP2 genes were isolated from a killifish liver cDNA library. Sequence analyses showed that one is related to CYP2K1 previously identified in trout (Buhler et al., 1994). The other cDNAs were classified into two new subfamilies, CYP2N and CYP2P, with one gene in CYP2N (CYP2N1) and two genes in CYP2P (CYP2P2 and CYP2P3). Analyses of killifish RNA demonstrated that CYP2K2, CYP2P2, CYP2P3, and CYP2N1 transcripts are expressed in both liver and gut tissues. CYP2N1 transcripts also are expressed in heart and brain. Subsequently, a killifish heart cDNA library was screened with CYP2N1, and another CYP2N gene was isolated. Cardiac CYP2N2 shares $75 \%$ amino acid identity with liver CYP2N1 and is expressed most highly in heart and brain. CYP2P and CYP2N gene expression was examined in fish that were held without food and in fish that were treated with the diacylglycerol mimic, 12-O-tetradecanoyl phorbol-13-acetate (TPA) (Kraft and Anderson, 1983). RNA analyses of these treated fish showed an apparent down-regulation of expression of the CYP2P and CYP2N transcripts in the extra-hepatic tissues of the TPA treated fish. In addition, a CYP2P gene was isolated from a killifish genomic DNA library. Sequence analysis indicates that the intron-exon pattern for this gene, CYP2P1, is similar to that of other CYP2 genes (Suwa et al., 1985). Altogether, these six killifish CYP2 genes in three different subfamilies suggest a diversity and multiplicity of CYP2 genes in early diverging vertebrates. 


\section{INTRODUCTION}

The cytochrome P450 (CYP) superfamily contains both a great number and a great diversity of proteins. These proteins are found in most if not all phyla, and they have key functions in toxicology, endocrinology, pharmacology, and carcinogenesis. Many CYP

proteins such as aromatase (CYP19) have similar functions in different species (Evans et al., 1987) while others such as P450cam have specialized functions in particular species (Sligar and Murray, 1986). Knowledge of the similarities and differences between diverse CYP proteins is fundamental to understanding the CYP superfamily as a whole.

The CYP2 family is the largest family in the cytochrome P450 superfamily. Currently, it has fifteen subfamilies, and about $20 \%$ of all CYP sequences are in family 2 (Nelson et al., 1996). The CYP2 proteins in mammals metabolize a wide array of both endogenous and exogenous substrates. For example, CYP2A1 in rats is a testosterone $7 \alpha-$ hydroxylase (Sonderfan et al., 1989). The CYP2Bs in several species are chlorobiphenyl hydroxylases (Ryan et al., 1982). Human CYP2C8 and CYP2J2 are arachidonic acid epoxygenases (Rifkind et al., 1995; Wu et al., 1996). CYP2D6, also in humans, activates opiates (Kerry et al., 1994). CYP2E1 in rats metabolizes ethanol, ketones, benzene, and trichloroethylene (Nanji et al., 1994). Both the number and the diversity of the CYP2 subfamilies in mammals suggest that divergent ecological or physiological pressures may have influenced this gene family and its expression. Determining whether other vertebrate groups have a similar diversity of CYP2 genes may establish when in phylogeny this gene radiation occured and may indicate those ecological or physiological features contributing to this diversity.

Fish constitute the largest and earliest diverging vertebrate group. They first evolved $500+$ million years ago, roughly 300 million years before mammals (Nelson, 1984). Knowledge of CYP in early diverging vertebrates has important implications for 
understanding the evolution of vertebrate CYP in general. If CYP are highly conserved between fish and mammals, this would suggest conserved and possibly endogenous functions. On the other hand, wide differences in CYP between fish and mammals may underlie fundamental organismal differences. Finally, sequence analysis of CYP in different vertebrates addresses the evolution of this gene superfamily in vertebrates.

The myriad CYP in the CYP2 family are postulated to have arisen in order to metabolize dietary plant natural products (Nelson and Strobel, 1987). Unlike mammals, the majority of fish are carnivorous and encounter fewer dietary plant natural products (Horn, 1989). In fact, in the temperate carnivorous fish most studied, total CYP levels are not induced by the prototypic CYP2 inducer, phenobarbital (Kleinow et al., 1987; Elskus and Stegeman, 1989; Elskus and Stegeman, 1989; Kleinow et al., 1990), and this lack of induction has been postulated to reflect the lack of terrestrial plants in their diets (Stegeman, 1993). If CYP2 proteins first evolved to metabolize endogenous substrates and later expanded and diversified in order to cope with dietary plant natural products, then fish that have not had to cope with dietary plant natural products may have less diversity of CYP2 proteins. Thus, fish may be a good vertebrate system with which to examine interactions between CYP and endogenous substrates, and knowledge of fish CYP2 proteins might have important implications for identifying endogenous CYP2 substrates.

Several lines of evidence indicate that fish have multiple CYP2 proteins. In numerous fish species, immunoblot analyses have shown CYP2B-like and CYP2E-like proteins (Stegeman, 1989; Stegeman and Hahn, 1994; Kaplan et al., 1991). As well, the lauric acid hydroxylases, CYP2K1 and CYP2M1 have been isolated from trout, and again, immunoblot analyses have shown CYP2K1-like and CYP2M1-like proteins in other fish species (Buhler et al., 1994; Yang et al., 1995). Whether fish have either orthologues or paralogues of many mammalian CYP2 proteins and whether unique CYP proteins occur in fish is not known. 
Our present objectives are to examine the diversity of CYP2 genes in the marine fish Fundulus heteroclitus. Multiple CYP2 proteins have been studied in mammals, but less is known about the diversity, number and functions of non-mammalian CYP2 proteins. Since the profusion of CYP2 subfamilies obscures the functional relationships between CYP2 proteins, establishing the diversity of non-mammalian CYP2 proteins and their relationships to known CYP2 proteins may clarify these functions.

\section{MATERIALS AND METHODS}

Materials: $\left[\alpha^{32} \mathrm{P}\right] \mathrm{dCTP}$ was purchased from Amersham, and $\left[\gamma^{32} \mathrm{P}\right] \mathrm{ATP}$ was purchased from DuPont NEN. Restriction enzymes, T4 DNA ligase, T4 polynucleotide kinase, and the Nick Translation System were purchased from Promega. Infra-red dye labelled sequencing primers were purchased from Licor. Long Ranger acrylamide was purchased from FMC Biochemicals. Cycle sequencing kits were purchased from Epicentre Technologies. General contractor cloning kits were purchased from 5 Prime to 3 Prime. Bluescript vector was purchased from Stratagene. Primers were purchased from Operon. RNA Stat-60 was purchased from Tel-Test "B," Inc. All other chemicals and reagents were purchased from Sigma unless otherwise noted.

Screening of the libraries: One killifish liver cDNA library was constructed in lambda gt10 from a single female fish liver. It had an amplified titer of $1.5 \times 10^{8} \mathrm{pfu} / \mathrm{ml}$ and average insert size of $1.0 \mathrm{~kb}$. A second killifish liver cDNA library was constructed in Lambda Zap from a pool of female fish. It had an amplified titer of $1.6 \times 10^{10} \mathrm{pfu} / \mathrm{ml}$ and average insert size of $1.8 \mathrm{~kb}$. The killifish heart cDNA library was constructed in Lambda Zap from a pool of male and female hearts. It had an amplified titer of $1.5 \times 10^{10} \mathrm{pfu} / \mathrm{ml}$ and average insert size of $1.5 \mathrm{~kb}$. The killifish genomic library was constructed in Lambda Fix II from a male fish (Stratagene). It had an amplified titer of $5 \times 10^{9} \mathrm{pfu} / \mathrm{ml}$ and insert 
sizes from $9-23 \mathrm{~kb}$.

Approximately $5 \times 10^{5}$ phage from the lambda gt10 liver cDNA library were plated with XL1-Blue E. coli as host and screened as described (Sambrook et al., 1989). These were screened with two degenerate probes end-labelled with $\left[\gamma^{32}\right.$ P]ATP. The oligonucleotide probes were designed based on conserved regions found in multiple CYP2 proteins from multiple species (Hobbs et al., 1986; Graves et al., 1990; Gotoh, 1992). The CYP2 proteins were rat 2A1, 2B1, 2B2, 2G1, 2D1, and 2E1, mouse 2A5, human $2 \mathrm{~B} 1$ and $2 \mathrm{~F} 1$, rabbit $2 \mathrm{~B} 4,2 \mathrm{C} 3$, and $2 \mathrm{C} 4$, dog $2 \mathrm{C} 21$, chicken $2 \mathrm{H} 1$ and $2 \mathrm{H} 2$, and $P$. putida 101A1. The primers were based on conserved regions corresponding to alpha helices in CYP101A1 as determined by x-ray crystallography (Gotoh, 1992). Thus, they likely code for structural parts of the protein which probably are more conserved than substrate binding regions. The 5 ' end CYP2 probe had a PstI and a XhoI restriction enzyme recognition sequence at its $5^{\prime}$ most end. Its sequence was

5'-CTGCAGCTCGAGGAGCGVATYCAGGASGARGC-3'. The 3' end CYP2 probe had a BamHI and a Xbal restriction enzyme recognition sequence at its 5 ' end . Its sequence was 5'-GGATCCTCTAGATSACBGCVTCNGTGTAKGGCA-3'. Where appropriate, codon usage tables for teleosts were used to eliminate degeneracies (Fitzgerald et al., 1993).

Hybridizations were done in 6XSSPE, $0.05 \mathrm{X}$ Blotto, and $20 \%$ formamide at $42^{\circ} \mathrm{C}$ overnight. Approximately 90 positive clones were identified. 35 of these were subcloned into pBluescript $\mathrm{SK}(+)$ and cycle sequenced by the dideoxy chain termination method (Sanger et al., 1977) using DNA polymerase (Epicentre) and infra-red labelled primers (Licor). Approximately $6 \times 10^{5}$ phage from each of the Lambda Zap liver and heart cDNA libraries were screened with the CYP2K2, CYP2P2, and CYP2N1 fragments isolated from the lambda gt10 library. These fragments were random primer-labelled with $\left[\alpha^{32} \mathrm{P}\right] \mathrm{dCTP}$ using Rediprime (Amersham). Hybridizations were done in 6XSSPE, 0.05X Blotto and $50 \%$ (liver) or $20 \%$ (heart) formamide at $42^{\circ} \mathrm{C}$ overnight. Positive clones were isolated 
from each library, rescued into pBluescript SK(+) (Stratagene), and sequenced as above.

Approximately $5 \times 10^{5}$ phage from the lambda Fix II library were plated with XL1Blue E. coli as host. Probes and hybridization conditions were identical to those used for the lambda gt 10 library. The $14 \mathrm{~kb}$ CYP2P1 clone was restriction enzyme digested, subcloned in pBluescript $\mathrm{SK}(+)$, and sequenced as above.

Sequence Analyses: The CYP2A, CYP2B, CYP2C, CYP2D, CYP2E, CYP2F, CYP2G, CYP2H, CYP2J, CYP2K, CYP2L, CYP2M, and CYP2Q genes were retrieved from the GenBank database. Accession numbers were: rat CYP2A1 (M33312), rabbit CYP2B1 (M20857), rat CYP2C23 (U04733), mouse CYP2D10 (M27168), hamster CYP2E1 (D17449), mouse CYP2F2 (M77497), rat CYP2G1 (M33296), chicken CYP2H2 (M25469), rabbit CYP2J1 (D90405), trout CYP2K1 (L11528), lobster CYP2L1 (U44826), trout CYP2M1 (U16657), and frog CYP2Q1 (D50560). The killifish sequences were assembled and translated using MacVector/AssemblyLign sequence analysis software (Oxford Molecular Group). The killifish nucleotide and deduced amino acid sequences were aligned with the CYP2 published sequences using CLUSTAL (Higgins and Sharp, 1988); refinements to the alignment were made manually. Phylogenetic trees based on amino acid alignments and nucleotide alignments (all three positions) were constructed using the minimum evolution criterion (Neighbor-Joining algorithm (Saitou and Nei, 1987)) and maximum parsimony (PAUP 3.1 (Swofford, 1990)). Phylogenetic trees based on nucleotide alignments also were constructed using distance-matrix (Olsen, 1988). Bootstrap analyses (Felsenstein, 1985) were performed to assess relative confidence in the topologies obtained.

RNA Analyses: Total RNA was prepared from killifish tissues using RNA Stat-60 according to manufacturer's directions except DNA sometimes was precipitated by centrifugation of the first homogenate for 5 minutes at $12000 \mathrm{~g}$. $10 \mathrm{ug}$ of total RNA was 
denatured and electrophoresed on $1.0 \%$ agarose gels containing $0.41 \mathrm{M}$ formaldehyde and transferred to nylon membranes by downward alkaline capillary as described (Chomczynski, 1992) except 4 bridge sheets were used rather than 2 . The blots were hybridized with the CYP2K, CYP2N, and CYP2P subclones nick translation labelled with $\left[\alpha^{32} \mathrm{P}\right] \mathrm{dCTP}$. Hybridizations were at $42^{\circ} \mathrm{C}$ in $50 \%$ formamide, 6XSSPE, $1 \%(\mathrm{w} / \mathrm{v})$ SDS, and $100 \mathrm{ug} / \mathrm{ml}$ of heat-denatured calf-thymus DNA. Even loading and complete transfer of RNA were monitored by detection of ethidium bromide stain in the gels and on the filters. Correlation between the stain on the filters and the amount of RNA was ascertained by probing the filters with labelled ribosomal RNA (data not shown).

Experimental Treatments Including TPA and Starvation: Sixty female fish that had been held in clean water for greater than six months were acclimated from $20^{\circ} \mathrm{C}$ to $14^{\circ} \mathrm{C}$ for 1 week. Six fish were sacrificed immediately and liver, gut, kidney, heart, and brain tissues were frozen immediately in liquid nitrogen for RNA analyses. Gut was divided into three equal parts: the fore-gut, the mid-gut, and the hind-gut. Twenty-four of the fish were fed excess TetraMin (Tetra Sales, Blacksburg, VA) throughout the experiment. The rest were held without feeding. After ten days, six fish from each group were sacrificed and liver, gut, kidney, heart, and brain tissues were frozen immediately in liquid nitrogen for RNA analyses. After two weeks, six fish from both groups were dosed intraperitoneally (ip) with $0.02 \mathrm{ug}$ TPA/g fish in $80 \%$ normal saline $/ 19.8 \%$ acetone $/ 0.2 \%$ ethanol (solvent), six fish from both groups were dosed ip with solvent, six fish from both groups were untreated, and six of the starved fish were fed excess TetraMin. Fish were sacrificed 72 hours later and liver, gut, kidney, heart, and brain tissues were frozen immediately in liquid nitrogen for RNA analyses. All tissues except liver and fore-gut were pooled. RNA from fore-gut was degraded and was not analyzed. 


\section{$\underline{\text { RESULTS }}$}

Molecular Cloning of Killifish CYP2 Genes: Screening killifish genomic and cDNA libraries with oligonucleotide primers designed to conserved regions in CYP2 genes identified six gene sequences in the CYP2 family. Five were cDNA sequences, and one was a genomic sequence. Sequence comparisons with known CYP2 genes showed that one of the killifish genes is related to the trout CYP2K1 gene described by Buhler et al. (Buhler et al., 1994). This killifish gene was designated CYP2K2 and was isolated from a liver cDNA library. The other genes were classified into two new subfamilies, CYP2N and CYP2P. CYP2N1, CYP2P2, and CYP2P3 were isolated from a liver cDNA library, CYP2N2 was isolated from a heart cDNA library, and CYP2P1 was isolated from a genomic DNA library. The sequences of CYP2K2, CYP2N1, CYP2N2, CYP2P1, CYP2P2, and CYP2P3 are shown in Figures 1-6.

Sequence Analysis: The CYP2K2 cDNA is $1.8 \mathrm{~kb}$ including $5^{\prime}$ and $3^{\prime}$ noncoding domains, and the coding region is 1518 base pairs. The CYP2P2 cDNA is $2.1 \mathrm{~kb}$ and the CYP2P3 cDNA is $1.9 \mathrm{~kb}$ including 5' and $3^{\prime}$ noncoding domains. The coding regions of both CYP2P2 and CYP2P3 are 1494 base pairs. Similarly, the CYP2N1 cDNA is $2.1 \mathrm{~kb}$ and the CYP2N2 cDNA is $2.4 \mathrm{kbp}$ including $5^{\prime}$ and $3^{\prime}$ noncoding domains while the coding regions for both are 1491 base pairs.

Both CYP2K2 and CYP2N2 have two putative start sites in the same reading frame, but CYP2K2 has a more favorable context around the first AUG initiator codon with a purine in position $-3(\mathrm{G})$ similar to $97 \%$ of vertebrate mRNAs and a $\mathrm{G}$ in position +3 similar to $46 \%$ of vertebrate mRNAs, and CYP2N2 has a more favorable context around the second AUG initiator codon with a purine (A) in position -3 (Kozak, 1991a; Kozak, 1991b). Additionally, the second AUG start site in the CYP2N2 sequence gives a 
predicted amino acid length more similar to the length of other CYP2 proteins and the same length as that predicted by the CYP2N1 gene.

The CYP2K2 and CYP2P3 cDNAs have the typical polyadenylation signal of AATAAA (underlined in Figures 1 and 5). However, this signal is not found in the CYP2P2 gene or the CYP2N genes; the most frequent variant polyadenylation signal of ATTAAA (Wahle and Walter, 1992) (underlined in Figures 2, 3, and 4) is present in all of these sequences. The instability factor ATTTA (Shaw and Kamen, 1986) is present in the 3'-ends of CYP2N1 (2 are present), CYP2N2 and CYP2P2. This sequence destabilizes transcript and may function in the regulation of these CYP.

CYP2P1 gene structure and transcription elements: The total length of the CYP2P1 gene is $\sim 14 \mathrm{~kb}$ and is separated into nine exons by eight intervening sequences. The ninth exon has not yet been sequenced although it has been mapped to the last $2.5 \mathrm{~kb}$ of the CYP2P1 subclone. All sequence comparisons for CYP2P1 were done with the first eight exons and so only approximate comparisons that will be done for the entire gene. The intron-exon pattern for CYP2P1 is similar to that of other CYP2 genes, and all the splicing junctions conform to the GT/AG rule (Breathnach and Chambon, 1981) (Table 1, Figure 7).

The 5' promoter region of CYP2P1 was analyzed for putative transcription elements using MatInspector (Quandt et al., 1995). This search method identifies potential regulatory motifs by automatic searches with a library of pre-compiled nucleotide distribution matrices of regulatory consensus sequences. Similar to many CYP2 sequences, the 5 promoter region of CYP2P1 contains five putative Barbie boxes (barbiturate responsive elements (Fulco et al., 1994)). Other putative regulatory sequences included multiple activator protein binding sites as well as sites for interferon regulatory factors (Tanaka N., 1993), estrogen receptors, sterol regulatory element binding proteins 1 (Kim et al., 1995), and NFkappa B 65 (Kunsch C., 1992). 


\begin{tabular}{|c|c|c|}
\hline Intron & Exon & Size $(\mathrm{kb})$ \\
\hline \multirow[t]{2}{*}{1} & & 0.2 \\
\hline & 1 & 1.8 \\
\hline \multirow[t]{2}{*}{2} & & 0.16 \\
\hline & 2 & 0.14 \\
\hline \multirow[t]{2}{*}{3} & & 0.15 \\
\hline & 3 & 1.58 \\
\hline \multirow[t]{2}{*}{4} & & 0.16 \\
\hline & 4 & 1.12 \\
\hline \multirow[t]{2}{*}{5} & & 0.18 \\
\hline & 5 & 0.82 \\
\hline \multirow[t]{2}{*}{6} & & 0.14 \\
\hline & 6 & 0.15 \\
\hline \multirow[t]{2}{*}{7} & & 0.19 \\
\hline & 7 & 1.8 \\
\hline \multirow[t]{2}{*}{8} & & 0.14 \\
\hline & 8 & $>3.5$ \\
\hline
\end{tabular}

Table 1. CYP2P1 intron and exon sizes in kilobases (kb). 


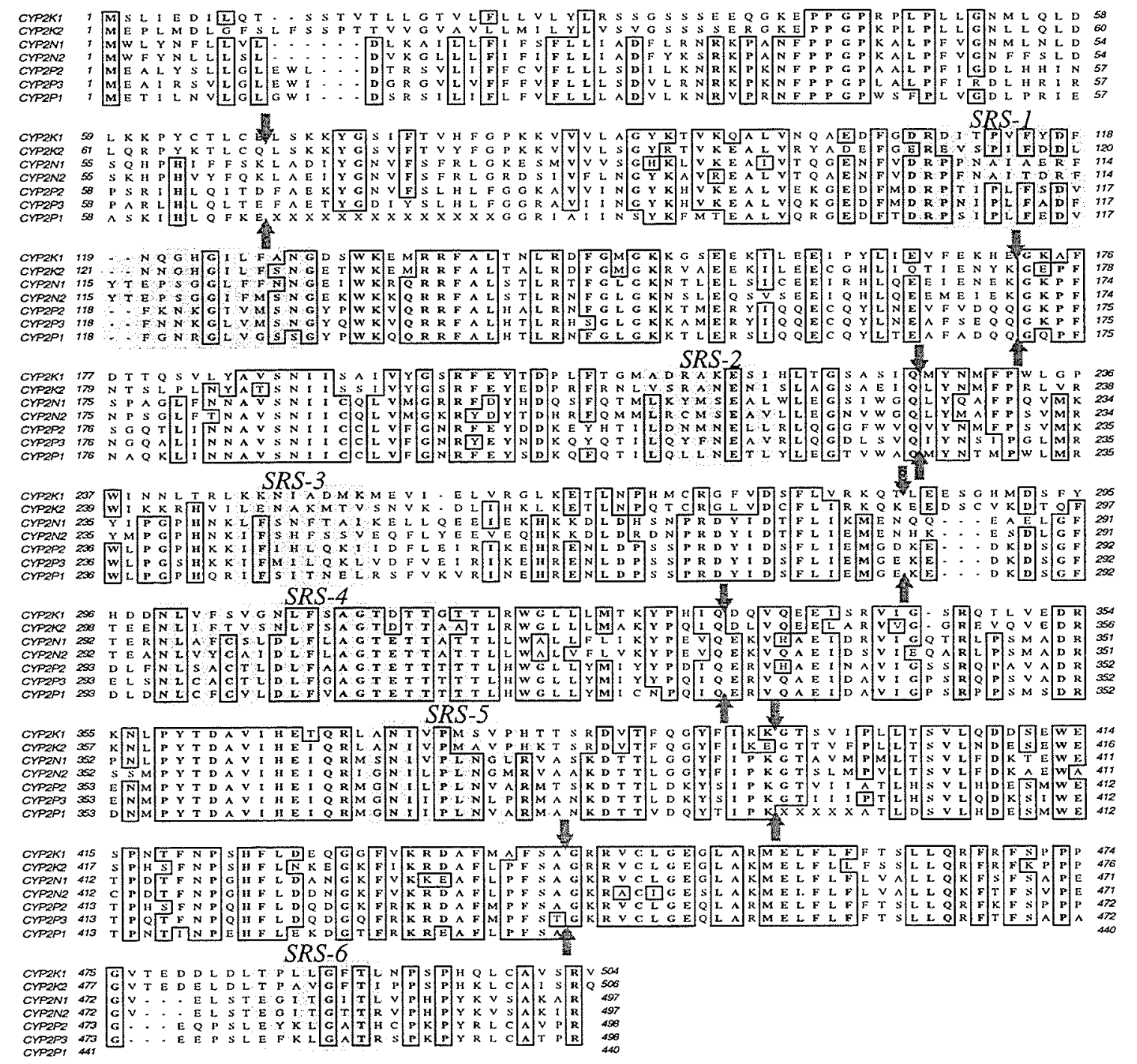

Figure 7. Deduced amino acid alignment of killifish CYP2K2, CYP2N1, CYP2N2, CYP2P1, CYP2P2, and CYP2P3 with trout CYP2K1. Sequences were aligned with CLUSTAL (Higgins and Sharp, 1988) and adjusted manually. Substrate recognition sites (SRS-1-6) as according to Gotoh (Gotoh, 1992) are shaded. Residues common to 5 or more sequences are boxed. The CYP2P1 intron/exon junctions are indicated with arrows. 
Nucleotide Comparisons: Figures 1-6 present the nucleotide sequences of the six CYP2 genes. All sequences contain the characteristic heme binding motif of cytochrome P450 genes (underlined in Figures 1-6). Length variation occurs in non-coding regions at both the $5^{\prime}$ and $3^{\prime}$ ends. Only coding regions were aligned and included in sequence comparisons. The CYP2P nucleotide sequences share 77-83\% identity with each other, 55-57\% identity with the CYP2Ns, and 50-52\% identity with the CYP2Ks. The CYP2N nucleotide sequences share $80 \%$ identity with each other and $49-51 \%$ identity with the CYP2Ks. The CYP2K2 nucleotide sequence shares 68\% identity with trout CYP2K1 (Table 2a)

Amino Acid Comparisons: Figure 7 presents the predicted protein sequences of teleost CYP2K1, CYP2K2, CYP2N and CYP2P genes in an alignment. Putative substrate recognition sites are shaded. CYP2P1 intron/exon junctions are indicated with arrows. The CYP2P predicted protein sequences share $71-81 \%$ amino acid identity with each other, 50-53\% amino acid identity with the CYP2Ns, and 40-48\% amino acid identity with the CYP2Ks. The CYP2N predicted protein sequences share $75 \%$ amino acid identity with each other and $42-45 \%$ amino acid identity with the CYP2Ks. The CYP2K2 predicted protein sequence shares 66\% amino acid identity with trout CYP2K1 (Table 2b). Comparisons between the killifish CYP2 genes and mammalian CYP2 genes show that the CYP2N and the CYP2P predicted proteins share $48-50 \%$ amino acid identity with the rabbit CYP2J1 and that the CYP2K2 predicted protein shares $42 \%$ amino acid identity with CYP2J1.

Phylogenetic analyses of CYP2 genes: Two sets of phylogenetic analyses were done. The first set of analyses used the majority of the coding sequences to infer the phylogenetic relationships between the CYP2 genes. The second set used only the six regions thought to be involved in substrate recognition as determined from the crystal structure of P450cam (Gotoh, 1992) to further examine these relationships. In the first 
Table 2a.

Percent nucleotide identities between killifish CYP2K2, CYP2N1, CYP2N2, CYP2P1, CYP2P2, and CYP2P3, trout CYP2K1, and rabbit CYP2J1.

\begin{tabular}{|c|c|c|c|c|c|c|c|c|}
\hline & CYP2K1 & CYP2K2 & CYP2N1 & CYP2N2 & CYP2P1 & CYP2P2 & CYP2P3 & CYP2J1 \\
\hline CYP2K1 & 100 & $\begin{array}{c}68 \\
(66)\end{array}$ & $\begin{array}{c}51 \\
(41)\end{array}$ & $\begin{array}{c}49 \\
(36)\end{array}$ & $\begin{array}{l}52 \\
(50)\end{array}$ & $\begin{array}{c}52 \\
(51)\end{array}$ & $\begin{array}{c}51 \\
(48)\end{array}$ & $\begin{array}{c}47 \\
(43)\end{array}$ \\
\hline CYP2K2 & & 100 & $\begin{array}{c}50 \\
(41)\end{array}$ & $\begin{array}{c}49 \\
(37)\end{array}$ & $\begin{array}{c}51 \\
(46)\end{array}$ & $\begin{array}{c}51 \\
(47)\end{array}$ & $\begin{array}{c}50 \\
(48)\end{array}$ & $\begin{array}{c}46 \\
(47)\end{array}$ \\
\hline CYP2N1 & & & 100 & $\begin{array}{c}80 \\
(76)\end{array}$ & $\begin{array}{c}57 \\
(51)\end{array}$ & $\begin{array}{c}55 \\
(57)\end{array}$ & $\begin{array}{c}57 \\
(48)\end{array}$ & $\begin{array}{c}54 \\
(54)\end{array}$ \\
\hline CYP2N2 & & & & 100 & $\begin{array}{c}56 \\
(47)\end{array}$ & $\begin{array}{c}55 \\
(49)\end{array}$ & $\begin{array}{c}56 \\
(47)\end{array}$ & $\begin{array}{c}53 \\
(52)\end{array}$ \\
\hline CYP2P1 & & & & & 100 & $\begin{array}{c}77 \\
(69)\end{array}$ & $\begin{array}{c}79 \\
(73)\end{array}$ & $\begin{array}{c}55 \\
(49)\end{array}$ \\
\hline CYP2P2 & & & & & & 100 & $\begin{array}{c}83 \\
(80)\end{array}$ & $\begin{array}{c}54 \\
(54)\end{array}$ \\
\hline CYP2P3 & & & & & & & 100 & $\begin{array}{c}55 \\
(54)\end{array}$ \\
\hline CYP2J1 & & & & & & & & 100 \\
\hline
\end{tabular}

Percent nucleotide identities between the substrate recognition sites as defined by Gotoh (Gotoh, 1992) are in parentheses. Percent identities with CYP2P1 were calculated using only eight of the nine possible exons and 5 of the 6 putative substrate recognition sites. Sequences were aligned with CLUSTAL (Higgins and Sharp, 1988) and adjusted manually. 
Table $2 b$.

Percent amino acid identities between killifish CYP2K2, CYP2N1, CYP2N2, CYP2P1, CYP2P2, and CYP2P3, trout CYP2K1, and rabbit CYP2J1.

\begin{tabular}{|c|c|c|c|c|c|c|c|c|}
\hline & CYP2K1 & CYP2K2 & CYP2N1 & CYP2N2 & CYP2P1 & CYP2P2 & CYP2P3 & CYP2J1 \\
\hline CYP2K1 & 100 & $\begin{array}{c}66 \\
(62)\end{array}$ & $\begin{array}{c}45 \\
(31)\end{array}$ & $\begin{array}{c}42 \\
(29)\end{array}$ & $\begin{array}{c}41 \\
(36)\end{array}$ & $\begin{array}{c}48 \\
(37)\end{array}$ & $\begin{array}{c}48 \\
(37)\end{array}$ & $\begin{array}{c}41 \\
(29)\end{array}$ \\
\hline CYP2K2 & & 100 & $\begin{array}{c}45 \\
(32)\end{array}$ & $\begin{array}{c}42 \\
(32)\end{array}$ & $\begin{array}{c}40 \\
(32)\end{array}$ & $\begin{array}{c}44 \\
(35)\end{array}$ & $\begin{array}{c}42 \\
(35)\end{array}$ & $\begin{array}{c}42 \\
(35)\end{array}$ \\
\hline CYP2N1 & & & 100 & $\begin{array}{c}75 \\
(72)\end{array}$ & $\begin{array}{c}51 \\
(47)\end{array}$ & $\begin{array}{c}53 \\
(44)\end{array}$ & $\begin{array}{c}51 \\
(44)\end{array}$ & $\begin{array}{c}50 \\
(48)\end{array}$ \\
\hline CYP2N2 & & & & 100 & $\begin{array}{c}50 \\
(44)\end{array}$ & $\begin{array}{c}51 \\
(49)\end{array}$ & $\begin{array}{c}51 \\
(46)\end{array}$ & $\begin{array}{c}48 \\
(44)\end{array}$ \\
\hline CYP2P1 & & & & & 100 & $\begin{array}{c}71 \\
(65)\end{array}$ & $\begin{array}{c}73 \\
(69)\end{array}$ & $\begin{array}{c}48 \\
(52)\end{array}$ \\
\hline CYP2P2 & & & & & & 100 & $\begin{array}{c}81 \\
(75)\end{array}$ & $\begin{array}{c}48 \\
(50)\end{array}$ \\
\hline CYP2P3 & & & & & & & 100 & $\begin{array}{c}48 \\
(52)\end{array}$ \\
\hline CYP2J1 & & & & & & & & 100 \\
\hline
\end{tabular}

Percent amino acid identities between the substrate recognition sites as defined by Gotoh (Gotoh, 1992) are in parentheses. Percent identities with CYP2P1 were calculated using only eight of the nine possible exons and 5 of the 6 putative substrate recognition sites. Sequences were aligned with CLUSTAL (Higgins and Sharp, 1988) and adjusted manually. 
analyses, over $94 \%$ of the sequence alignment was used to infer the phylogenetic relationships between the CYP2 genes. The $5^{\prime}$ and $3^{\prime}$-most ends were excluded due to length variations and resulting regions of uncertain alignments. A total of 1422 nucleotide positions out of 1491 were compared, and 1267 sites ( $85 \%)$ were informative. A total of 472 amino acid positions out of 497 were compared, and 411 sites (87\%) were informative. In the second analyses, only the six substrate recognition sites were used to infer relationships (228 nucleotides, 76 amino acids). Here, 205 nucleotide sites (90\%) and $68(89 \%)$ amino acid sites were informative.

Trees were generated using both amino acid and nucleotide sequences. All three nucleotide positions were used in the nucleotide analyses. Both maximum parsimony and distance methods were used to construct the trees, and the killifish CYP sequences formed the same groups with all methods. Bootstrap analyses were done to assess these topologies, and they collapsed the parsimony trees. An assumption in parsimony analyses is that multiple events do not occur (Swofford et al., 1996), and this assumption may not be true for a highly diverse set of sequences such as the CYP2 sequences. The CYP2 family undoubtedly contains many paralogous genes, and once a sequence has duplicated, it may change rapidly before selective pressure on its new function slows its rate of change (Pearson, in progress). Neighbor joining trees are shown with bootstrap values $>60 \%$ for both neighbor joining and parsimony (Figures $8 \mathrm{a}$ and $8 \mathrm{~b}$ ). The trees show that CYP2K2 is most closely related to CYP2K1 and that the CYP2Ns and the CYP2Ps are most closely related to the CYP2Js.

Tissue Distribution: To determine the tissue distribution of the expression of these CYP2 genes, total RNA isolated from different tissues was probed with the full length CYP2K2, CYP2N1, CYP2N2, CYP2P2 and CYP2P3 cDNAs using high stringency conditions. These fish CYP2 probes gave multiple transcripts in different tissues. Individual fish showed differences in expression levels of these genes. No clear difference 


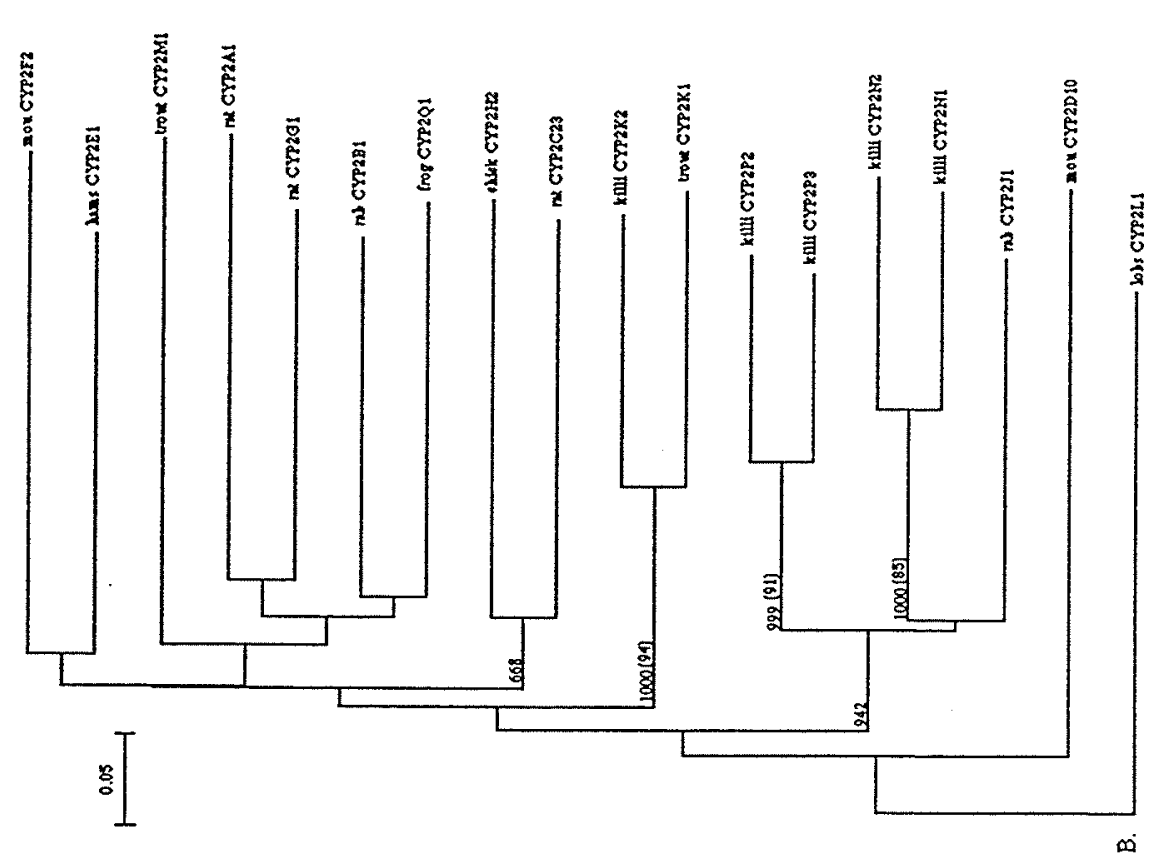

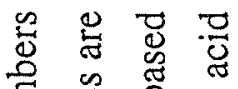

芝芯怘

远焉㐫

言 吉 官 号

की

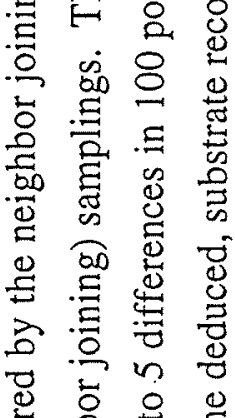

m

惫

엉 홍

\$ 8 \%

总范范

胥芯导忍

它蓄

递㤩

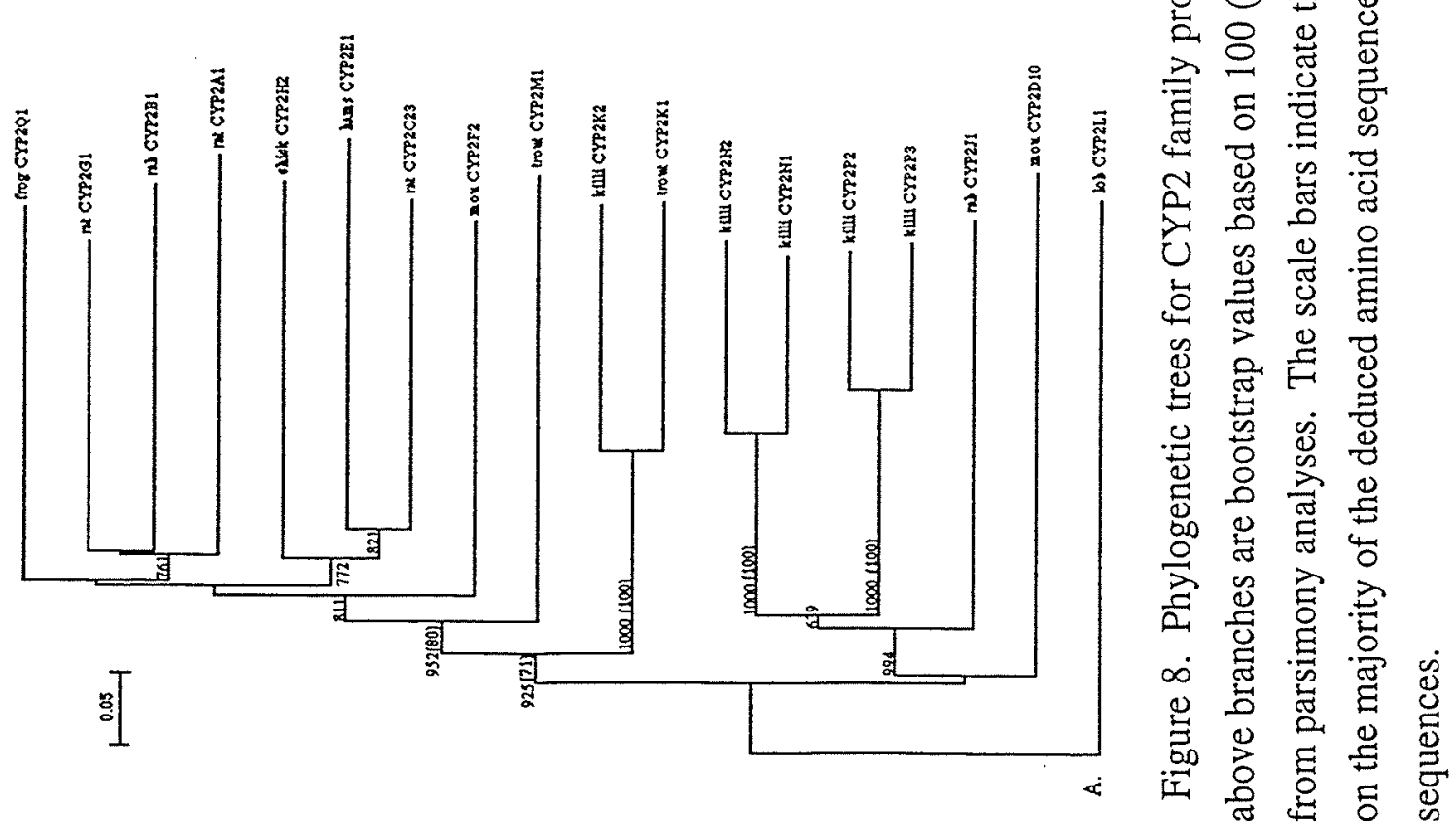


in expression was seen between male and female fish (Figures 9, 10, and 11).

The CYP2K2 probe hybridized with a single $1.8 \mathrm{~kb}$ band in liver and gut and appeared to be more highly expressed in the hind gut compared to the mid- and fore-gut . CYP2K2 cDNA also hybridized with a $1.8 \mathrm{~kb}$ band in ovaries (Figures 9, 10, and 11).

Probing RNA blots with the CYP2N1 cDNA revealed revealed multiple bands in some organs. There were three cross-hybridizing bands of approximately 3.0, 2.4, and $1.8 \mathrm{~kb}$ in both liver and gut (Figures 9 and 10). Only a $2.4 \mathrm{~kb}$ transcript was detected in the heart and brain tissues (Figure 11). Whether these three transcripts in liver and gut are separate genes or alternatively spliced variants is unknown. However, the $2.4 \mathrm{~kb}$ band detected with the CYP2N1 cDNA in heart and brain is the same size as the CYP2N2 transcript suggesting that CYP2N1 might be cross-hybridizing with CYP2N2 in these tissues. The CYP2N2 cDNA detected only a single band in several different tissues including heart, brain, liver, gut, eye, gill, and kidney (Figures 9, 10, and 11). This transcript was highly expressed in heart and brain and was expressed in liver and gut to lesser amounts. CYP2N2 probably did not cross-hybridize with CYP2N1 in liver and gut tissues because it only recognized a single band in these tissues rather than the three CYP2N1 cross-hybridizing bands. In addition, CYP2N2 was expressed only slightly in eye, gill, and kidney tissues.

In contrast to the CYP2Ns, the CYP2P2 and CYP2P3 transcripts were expressed only in gut and liver (Figures 9 and 10). The CYP2P2 probe hybridized with four bands in these tissues. The smallest of these bands was only $1.1 \mathrm{~kb}$ suggesting that it might be a degradation product. The other bands were $2.8,2.3$, and $2.0 \mathrm{~kb}$ in size. The CYP2P3 probe hybridized strongly with a 2.5 and a $1.6 \mathrm{~kb}$ band and very faintly with a $2.3 \mathrm{~kb}$ band. As with CYP2N1, whether these multiple CYP2P-like transcripts are separate genes or alternatively spliced variants is not known.

TPA/Fed/Starved Experiment: CYP2N and CYP2P transcript expression in liver 


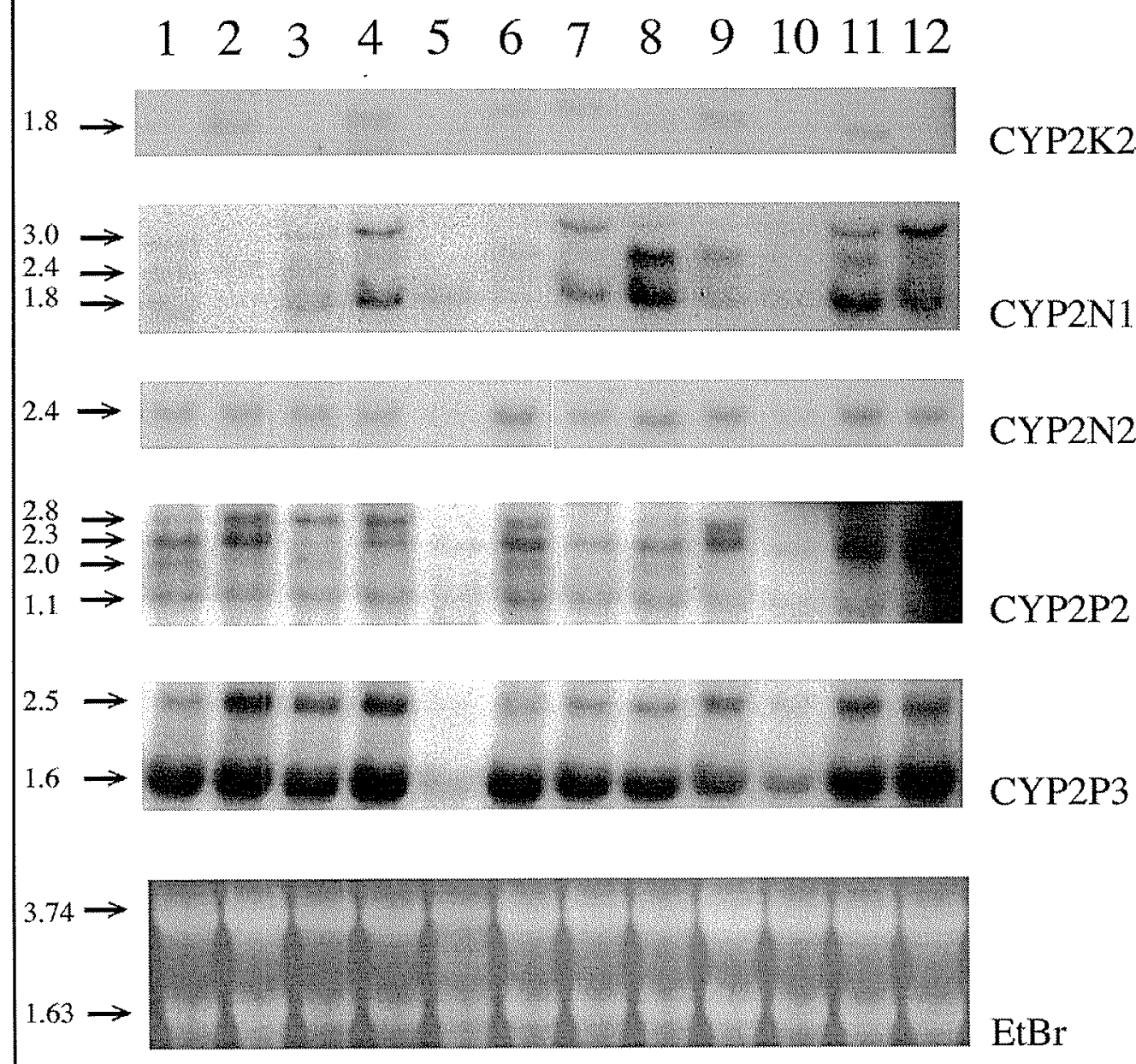

Figure 9. Northern analyses of male (1-6) and female (7-12) livers probed with CYP2K2, CYP2N1, CYP2N2, CYP2P2, and CYP2P3 cDNAs. Arrows show sizes in kilobases. Ethidium bromide (EtBr) shows relative loading. 


\section{$\begin{array}{llllll}1 & 2 & 3 & 4 & 5 & 6\end{array}$

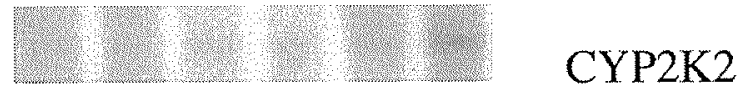

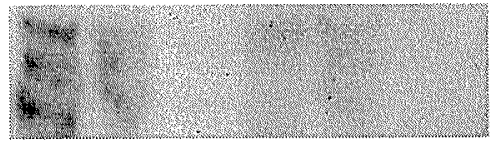

CYP2N1

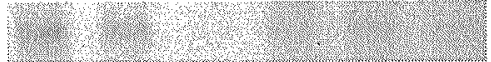

CYP2N2

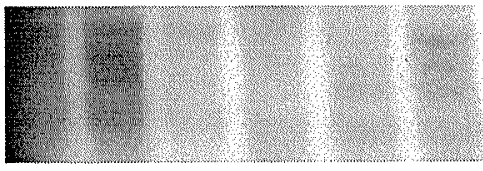

CYP2P2

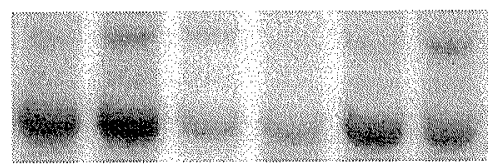

CYP2P3

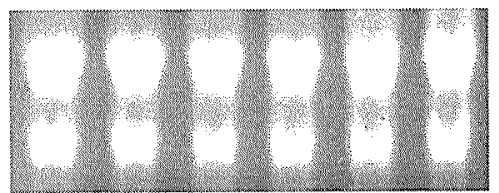

$\mathrm{EtBr}$

Figure 10. Northern analyses of male $(1-3)$ and female (4-6) fore-gut (1 and 4), mid-gut ( 2 and 5), and hind-gut (3 and 6) probed with CYP2K2, CYP2N1,CYP2N2, CYP2P2, and CYP2P3 cDNAs. Ethidium bromide (EtBr) shows relative loading. 

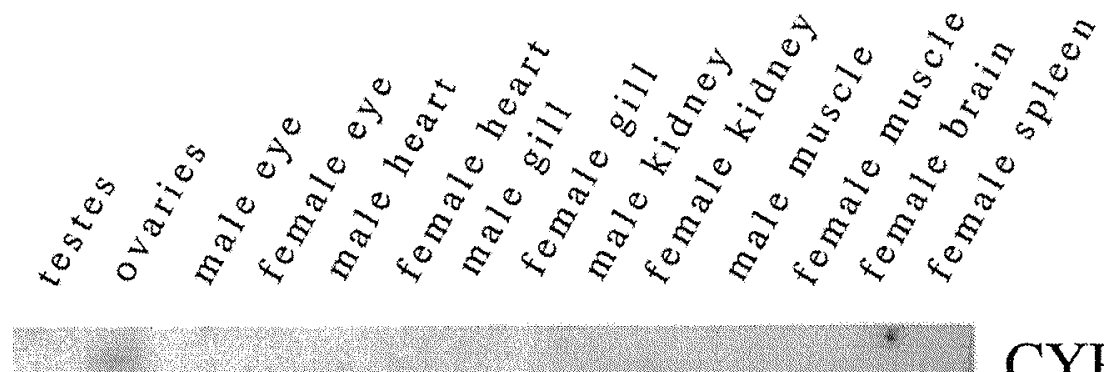

CYP2K2

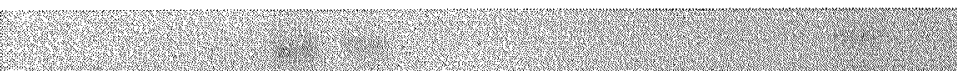

CYP2N1
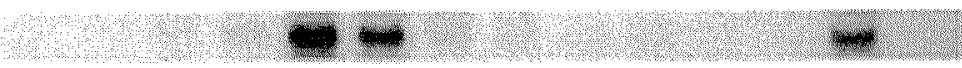

CYP2N2

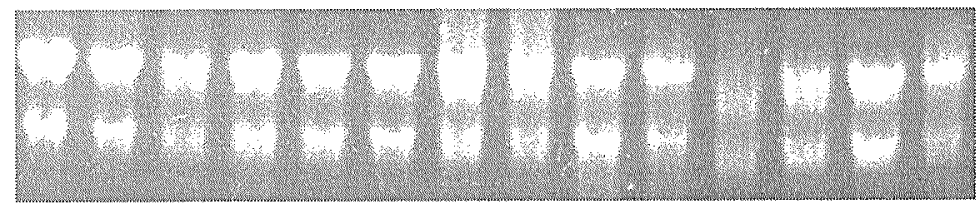

\section{$\mathrm{EtBr}$}

Figure 11. Northern analyses of male and female extra-hepatic tissues probed with CYP2K2, CYP2N1, and CYP2N2 cDNAs. Ethidium bromide (EtBr) shows relative loading. 
and gut suggests a possible dietary role for these CYP while their relationship to the CYP2Js suggests a possible involvement with arachidonic acid (see discussion). Thus, expression of CYP2P2, CYP2P3, CYP2N1 and CYP2N2 transcripts was examined in fed fish, in starved fish, and, because TPA can affect the concentration of free arachidonic acid (Fischer and Slaga, 1985), in TPA treated fish. In these treated fish, the tissue distribution of CYP2N and CYP2P expression did not change with any of the treatments. No consistent pattern of expression was seen in the livers of these fish which may reflect individual variation in hepatic expression of the CYP2N and CYP2P genes (variation in hepatic expression is clear in Figure 9). This variation precluded interpretations of individual response. However, in the pooled tissues, this variation was quenched, and TPA treatment appeared to down-regulate CYP2N and CYP2P transcript expression in all tissues but brain. As well, CYP2N2 transcript in heart appeared to be up-regulated in the fish that had been starved for twenty days and in the fish that had been starved for seventeen days and then fed once, three days before being killed (Figure 12).

\section{DISCUSSION}

The CYP2 gene family includes a bewildering array of genes that are expressed in a wide variety of organisms and metabolize an extraordinary array of both exogenous and endogenous substrates. Although the CYP2 family comprises the largest cytochrome P450 family, little is known about the diversity, number, and functions of CYP2 genes in nonmammalian vertebrates. Fish are the largest, most diverse, and earliest diverging vertebrate group. Analysis of fish CYP2 genes may provide insights into the functional relationships and possibly the basis for diversification among the CYP2s. In this study, multiple novel genes in the CYP2 family were identified and characterized in the killifish, Fundulus heteoclitus. Four sequences designated CYP2K2, CYP2N1, CYP2P2, and CYP2P3 were 


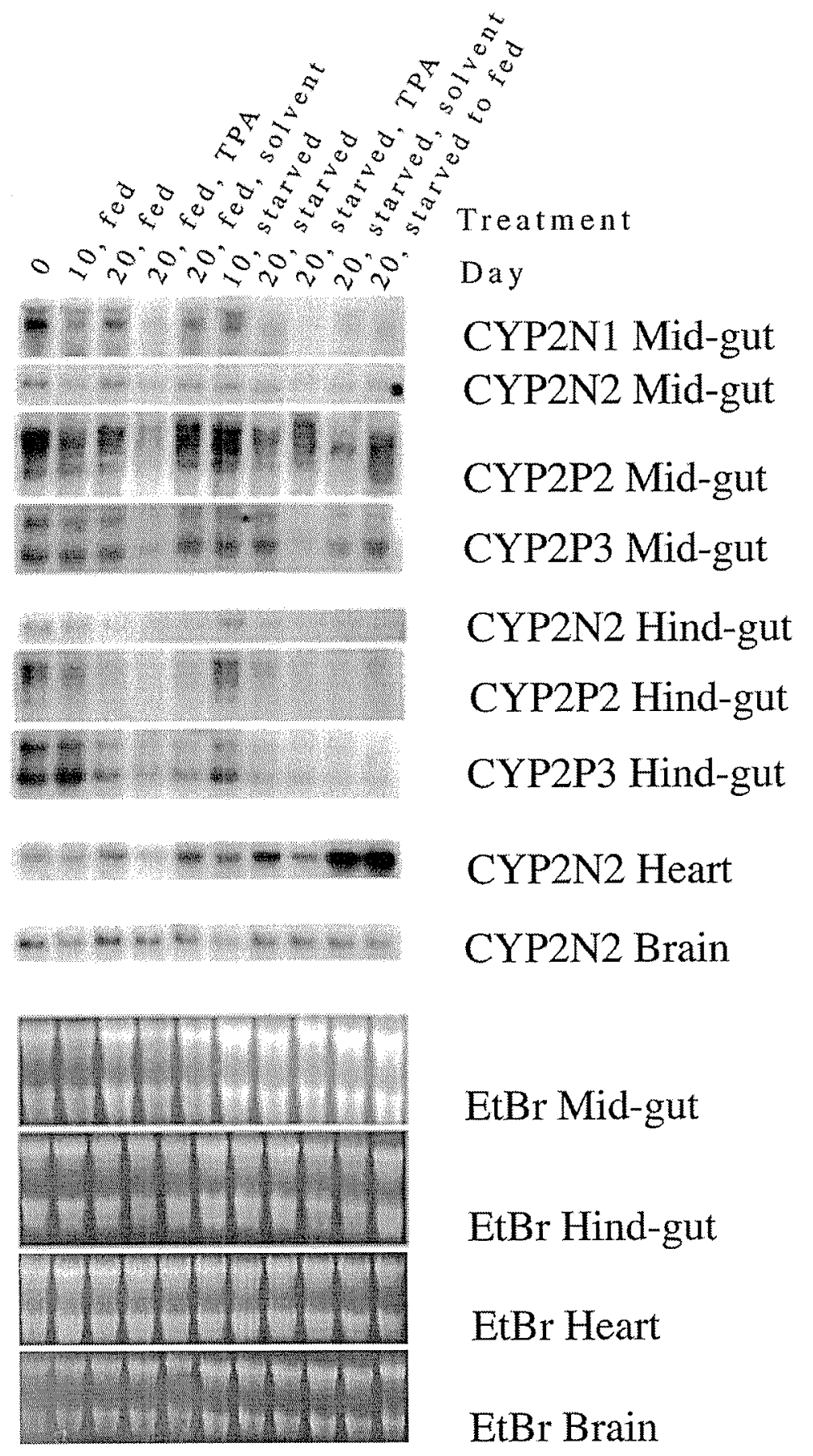

Figure 12. Northern analyses of extra-hepatic tissues of fed/starved/TPA treated fish probed with CYP2N1, CYP2N2, CYP2P2, and CYP2P3 cDNAs. Ethidium bromide (EtBr) shows relative loading. 
isolated from a liver cDNA library. CYP2N2 was isolated from a heart cDNA library, and CYP2P1 was isolated from a genomic DNA library.

\section{Sequence Structure}

The deduced amino acid sequences for these genes all contain putative heme binding peptides (Figures 1-6) with the conserved cysteine that donates the thiolate fifth ligand to the heme iron. All of these sequences also have the conserved PPGP sequence thought to be at the carboxyl end of the membrane anchor and which may act as a hinge region between the hydrophobic leader and the body of the protein (Yamazaki et al., 1993). The sizes of the CYP2K2 and CYP2N2 genes are the same sizes as the transcripts detected in RNA analyses suggesting that these sequences are full length transcripts. In contrast, the sizes of the CYP2N1, CYP2P2, and CYP2P3 genes are different than the sizes of the transcripts detected in RNA analyses. These size differences could be due to alternative splicing. Alternatively, these sequences might not be full length transcripts. Portions of the $5^{\prime}$ and $3^{\prime}$-untranslated transcripts may be missing. This is less likely for the $3^{\prime}-$ untranslated ends because polyadenylation sites were present in all of these genes. However, more than one polyadenylation signal could be present. While the CYP2K2 and the CYP2P3 genes have the typical polyadenylation signal of AATAAA, the most frequent variant polyadenylation signal of ATTAAA (Wahle and Walter, 1992) is present in the CYP2P2 and both CYP2N genes. Interestingly, atypical polyadenylation sequences are frequently found in CYP genes (Sogawa et al., 1985), and the variant polyadenylation signals found in CYP2N1, CYP2N2, and CYP2P2 suggest that atypical polyadenylation sequences also may be frequent in CYP genes in early diverging vertebrates.

CYP2P1, isolated from a genomic library, has nine exons with intervening intron sequence, a gene structure very similar to that of mammalian CYP2s (Suwa et al., 1985). Likely, the other fish CYP2 genes have this same gene structure. The size of the CYP2P1 
$(\sim 14 \mathrm{~kb})$ gene is similar to that of rat P450e but not rat P450b (14 kb vs $23 \mathrm{~kb}$ ) (Suwa et al., 1985). This size similarity does not reflect the relative genome sizes of these different species since the killifish genome is about one-third the size of the rat genome (Hinegardner and Rosen, 1972). Studies with pufferfish suggest that mammals and fish have the same number of genes (Brenner et al., 1993), and so the relatively large gene size of killifish CYP2P1 might reflect important regulatory regions of this gene. Because many CYP are regulated at the level of transcription of the mRNAs, we looked for possible regulatory features in the 5'-promoter region of the CYP2P1 gene.

Examination of the 5'-promoter region of CYP2P1 revealed a number of putative regulatory sequences. These included potential hormonal regulatory elements such as consensus sequences for the estrogen receptor and the sterol regulatory element binding protein (Kim et al., 1995) as well as activator elements such as AP-1 Fos/Jun and CCAAT enhancer binding protein $\beta$ (Akira et al., 1990). Interestingly, CCAAT enhancer binding protein $\beta$ is expressed in the liver at much higher levels that in other tissues and may activate the expression of genes expressed specifically in the liver (Latchman, 1991). Consensus sequences for interferon regulatory factor (Tanaka N., 1993) and NFkappaB 65 (Kunsch C., 1992) also were identified, suggesting that CYP2P1 might function in cell proliferation. Notably, although fish are not known to be responsive to barbiturates, multiple (5) Barbie box (barbiturate response elements) consensus sequences (Liang Q., 1995) were identified in the 5 '-promoter region. Intriguingly, the closely related CYP2P3 protein (73\% deduced amino acid identity with CYP2P1) has recently been shown to metabolize arachidonic acid (Oleksiak et al., in preparation), and it has been suggested that the Barbie box is responsive to fatty acids since fatty acids, in particular unsaturated fatty acids such as arachidonic acid, avidly bind to the BM3RI repressor (Wolf et al., 1996). This repressor interacts with Barbie boxes and is inhibited upon drug binding (Fulco et al., 1994). Determining whether or not these responsive elements function in the regulation of 
CYP2P1 might provide insight into the evolution and maintenance of CYP 2 family gene regulation.

\section{Sequence Comparisons}

The conservation of nucleotide sequence between the killifish CYP2K2 gene and the trout $\mathrm{CYP} 2 \mathrm{~K} 1$ gene is lower than that between teleost CYP1A sequences in different fish species. There is 68\% identity between the CYP2K sequences while there is $77-88 \%$ among various CYP1A sequences in teleosts (Morrison et al., 1995). This lesser conservation between likely orthologues may reflect the larger size of the CYP2 family which would present greater opportunities for homologous recombination between similar sequences. This homologous recombination results in gene homogenization or concerted evolution (Hood et al., 1975) so that members of a gene family within a species are more similar to each other than to orthologous genes within a different species.

This effect of concerted evolution is suggested by the clustering of the CYP2N and CYP2P genes in the distance trees (Figures $8 \mathrm{a}$ and $8 \mathrm{~b}$ ). The CYP2N nucleotide sequences are $80 \%$ identical to each other, $\sim 55 \%$ identical to the CYP2Ps, and $\sim 50 \%$ identical to the CYP2Ks. The CYP2Ps are $\sim 83 \%$ identical to each other and $\sim 51 \%$ identical to the CYP2Ks (Table 1a). These nucleotide identities all are higher than the corresponding amino acid identities (Table 2b). This is unusual. For most genes, the amino acid sequences are more similar than the nucleotide sequences. Notably, this higher nucleotide identity is not unique to these fish CYP2 genes. Inspection of published sequences shows that the same is true for CYP2s in different subfamilies as well as for CYP2s from species as distant as lobster and human (CYP2L1 vs. CYP2J2), suggesting that this aberration is not an artifact of either concerted evolution or codon usage.

The lower amino acid identity suggests that amino acid changes are not being selected against (resulting in the evolution of paralogous genes). Frequent amino acid 
changes might not be selected against if functional diversity were advantageous, as it would be when needing to deal with a variety of new compounds. Similar results (i.e. \% nucleotide identities $>\%$ amino acid identities) might be expected in other CYP families whose members metabolize a wide variety of substrates, such as the CYP3 and CYP6 families. In fact, CLUSTAL alignments (Higgins and Sharp, 1988) of published CYP3A and CYP6 nucleotide and deduced amino acid sequences show that the \% nucleotide identities also are greater than the \% amino acid identities for sequences in these CYP families. These results concur with the idea that the need to metabolize a variety of plant natural products has driven the evolutionary diversity of the CYP2 family (Nelson and Strobel, 1987).

Comparisons between the substrate recognition sites of the killifish CYP2 genes show that at the nucleotide level, the \% identities between the substrate recognition sites of the genes are lower than the \% identities of the entire gene (Table $2 \mathrm{a}$ ). At the amino acid level, the \% identities also are lower when only the substrate recognition sites are compared perhaps suggesting substrate differences. Interestingly, this is not true for the CYP2P compared to the CYP2J1 substrate recognition sites. In these genes, the \% identities over the substrate recognition sites are higher than those over the entire gene (Table $2 b$ ).

Phylogenetic analyses could identify closely related mammalian sequences which might suggest functional relationships for these fish CYP proteins even in a family as large and diverse as the CYP2 family. We did two types of analyses. We did phylogenetic comparisons with a representative member from each CYP2 subfamily using the majority $(>94 \%)$ of the sequence; we did similar comparisons using only the substrate recognition sites. The killifish CYP2 sequences formed the same groups with both the entire sequences and only the substrate recognition sites, using either maximum parsimony or distance methods. The topology of these trees showed that killifish CYP2K2 is related most closely to trout CYP2K1 and that the CYP2Ns and the CYP2Ps are related most 
closely to the mammalian CYP2Js. Although changes in even one amino acid can dramatically alter the substrate specificity of a particular CYP (Lindberg and Negishi, 1989), this grouping suggests that the trout and killifish CYP2Ks might share similar catalytic properties and that the CYP2Ns, the CYP2Ps and the CYP2Js also might share similar catalytic properties.

Tissue Distribution

The phylogenetic comparisons show that, as expected, CYP2K2 clearly is related most closely to trout CYP2K1 suggesting an orthologous relationship. (Trout CYP2K3 recently was identified (Buhler, unpublished data) and is $96.5 \%$ identical to CYP2K1 at the amino acid level. This high identity suggests that CYP2K1 and CYP2K3 are recently diverged. Thus, it seems likely that killfish CYP2K2 might be orthologous to both CYP2K1 and CYP2K3). However, the tissue distribution of these two genes is not identical. Both CYP2K1 and CYP2K2 are expressed in liver, and expression levels vary between individuals. CYP2K1 also is expressed in trunk kidney and seems to be downregulated in reproductively active female fish in both liver and kidney (Buhler et al., 1994). In contrast, no apparent difference was seen between CYP2K2 expression in male and female fish, and as well as being expressed in liver, CYP2K2 transcript also is expressed in gut, especially hind gut, and in ovaries. CYP2K1 has been shown to have estradiol 2hydroxylase activity (Miranda et al., 1989) which might explain the expression of CYP2K2 in killifish ovaries.

The CYP2P and CYP2N transcripts are expressed in gut and liver. Transcript levels of the CYP2N and CYP2P genes vary between individual, untreated fish. While some fish show high levels of expression, other fish show very little expression suggesting that these genes are not constitutive and instead may be somehow regulated. This regulation might be due to an external variable. For instance, CYP1A levels have been 
shown to be regulated by levels of environmental polycyclic aromatic hydrocarbons (Stegeman et al., 1981). In fact, induction of CYP1A often is used as a biomarker of environmental pollution (Stegeman et al., 1988; Elskus and Stegeman, 1989). In contrast, the distinction between an environmental factor and an endogenous factor is not as clear when considering CYP2E induction. Starvation, an external factor, causes the production of ketones, an endogenous factor, which induces CYP2E proteins (Koop, 1992). Hence, although interindividual variation in expression of the CYP2Ns and CYP2Ps might suggest some type of exogenous regulation, an alternative explanation is that an external factor affects an endogenous ligand which in turn regulates the levels of the CYP2Ns and the CYP2Ps. Finally, endogenous substrates such as hormones have been shown to regulate hepatic CYP isozyme expression (Waxman, 1988) and hormonal or a similar type of regulation is a third explanation for the interindividual variation in expression of the CYP2Ns and CYP2Ps. Levels of the CYP2N2 and CYP2P transcripts, but not of the CYP2N1 or CYP2K2 transcripts, covary between the different fish suggesting some type of coordinate regulation for the CYP2N2 and CYP2P genes. Catalytically, CYP2N1 and CYP2N2 are very similar in their metabolism of arachidonic acid (Oleksiak et al., in prep), and differential expression may reflect physiological, functional differences not yet evident in substrate profiles.

Intriguingly, CYP2N1 and CYP2N2 are expressed in both heart and brain. While CYP2N1 is only slightly expressed, CYP2N2 is highly expressed in these tissues. Since the evolution of the brain was dependent on the evolution of the vasculature for the supply of oxygen and nutrients, this might imply a coordinate regulation, function, or substrate for this gene in these two tissues. In addition, the gut is well innervated and well vascularized, and a function that involves supplying of nutrients might explain why the CYP2Ns also are expressed in gut. Knowledge of the cell types that express the CYP2N genes might elucidate any relationship concerning their expression in these different tissues. 
Although many CYP2 genes are involved in the metabolism of exogenous substrates, expression of the CYP2N and CYP2P genes in extrahepatic tissues, especially the predominant expression of CYP2N2 in heart and brain, suggests that these genes might be involved in the metabolism of endogenous substrates as well. Interestingly, CYP2J2 also is expressed in human liver, gut, and heart (Wu et al., 1996), CYP2J1 is expressed in rabbit small intestine (Kikuta et al., 1991), CYP2J3 is expressed most highly in liver and also in lung, kidney, stomach, and small intestine (Wu et al., 1997), and CYP2J4 is highly expressed in liver, small intestine, and olfactory mucosa and also in heart, lung, and kidney (Zhang et al., 1997). Thus, the CYP2Ns and CYP2Ps share both sequence similarities and tissue distribution similarities with the CYP2Js, suggesting putative functional similarities as well.

Recently, CYP2J2, CYP2J3, and CYP2J4 were shown to metabolize arachidonic acid (Wu et al., 1996; Wu et al., 1997; Zhang et al., 1997). The phylogenetic similarities between the CYP2Ns, the CYP2Ps and the CYP2Js as well as their similar tissue specific expression patterns suggest that the CYP2Ps and the CYP2Ns also might metabolize arachidonic acid. Functional analyses support this (Oleksiak et al., in preparation; Oleksiak et al., in preparation).

\section{TPA/Fed/Starved Experiment}

High level expression of CYP2P and CYP2N transcripts in both gut and liver suggests a possible dietary role for these genes. Such a role might involve either exogenous or endogenous substrates. For example, these genes might be expressed in order to metabolize exogenous compounds that are of dietary origin. Killifish are mainly carnivorous although algal filaments and detritus are common gut contents (D'Avanzo and Valiela, 1990). Thus, they potentially are exposed to a variety of plant natural products such as terpenes, phenols, and phorbol esters (Faulkner and Fenical, 1977). These 
expressed genes also might metabolize an endogenous compound. A dietary endogenous role might involve the vasculature, possibly involving vasoconstriction or vasodilation depending on the fullness of the gut. Increasingly, the importance of P450 metabolites of arachidonic acid for vasoactivity is being shown in mammals (Harder et al., 1995).

In order to examine possible dietary and/or endogenous roles for the CYP2Ns and CYP2Ps, we examined fish that were fed, fish that were starved, and fish that were treated with the plant phorbol ester TPA. TPA has been shown to alter the activity of protein kinase C (Kraft and Anderson, 1983) and thus phospholipase A2, consequently affecting the concentration of free arachidonic acid (Fischer and Slaga, 1985). A clear pattern that emerged from this TPA/fed/starved experiment was an apparent down-regulation in expression of the CYP2N and CYP2P transcripts in all TPA treated, extra-hepatic tissues except brain (Figure 12). This pattern is particularly obvious for CYP2P3 transcript expression in the midgut and for CYP2N2 transcript expression in the heart. Not surprisingly, the levels of CYP2N2 transcript in the brain remained constant, suggesting that the brain was protected from dietary changes and/or exposure to TPA. The extrahepatic tissues were pooled, and inter-individual variation in expression that precluded interpretations of liver samples (data not shown), likely are masked in these pools and might mask more striking regulatory responses. Nevertheless, TPA treatment appears to have an inhibitory effect on transcript expression. The similar responses of CYP2N2 and the CYP2Ps reinforce the idea that CYP2N2 and the CYP2Ps might be coordinately regulated.

Another effect was seen on CYP2N2 transcript expression in the heart. CYP2N2 transcript was up-regulated in the heart of fish that had been starved for twenty days and of fish that had been starved for seventeen days and then fed three days before being killed (starved to fed fish). This is particularly striking when compared to the other tissues, except brain, in which the starved fish appear to express less CYP2N and CYP2P 
transcript. These results suggest that lack of food might up-regulate the expression of CYP2N2 in heart. The fact that this induction was not lessened in the starved to fed fish might reflect a time lag in response. Alternatively, it might suggest that lack of food is not causing the induction. A number of other CYP are induced by starvation (e.g. CYP2B, CYP2E, and CYP3A (Soucek and Gut, 1992)), but none of these is expressed predominantly in the heart. The heart mainly relies on fatty acids, both esterified and free, for fuel, and during starvation, certain tissues such as kidney and skeletal muscle change their primary fuel substrate from glucose to fatty acids and ketone bodies in an effort to preserve protein (Isselbacher et al., 1994). Such physiological changes in fat balance might cause a concurrent increase in proteins that are involved in fatty acid metabolism in those tissues that can metabolize fatty acids as fuel, one function of the CYP2Ns (Oleksiak et al., in preparation). The physiological significance of this induction is not yet known.

Altogether, six CYP2 genes were isolated from killifish genomic and cDNA libraries. Given the variety and number of mammalian CYP2 proteins and the data presented here, it appears that fish also have diverse and numerous CYP2 proteins. Phylogenetic analyses of these sequences show that they belong to three different CYP2 subfamilies: CYP2K, CYP2N, and CYP2P. The functional significance of these genes is suggested by their sequence and expression similarities to other CYP2 genes, but remains to be determined. These results suggest a diversity of CYP2 genes present in fish with probable corresponding functional diversities. 
Figure 1. Nucletoide and predicted amino acid sequence for killifish CYP2K2. The start codon and stop codons are italicized. The putative polyadenylation signal (AATAAA or ATTAAA) is underlined. The heme binding motif characteristic of CYP proteins is underlined. Coding sequence is upper case. Non-coding sequence is lower case. Numbers in the right margin correspond to nucleotide positions. 
ATG GAG CCT TTA ATG GAT TTA GGT TTT TCT CTC TTC TCC AGT CCT ACC ACT GTG GTG GGA 85

$\begin{array}{llllllllllllllllllll}M & E & P & L & M & D & L & G & F & S & L & F & S & S & P & T & T & V & V & G\end{array}$ GTA GCT GTG CTT TWG ATG ATC CTT TAT TTG GTT TCT GTC GGT TCC TCC TCC AGC GAA CGA 145 $\begin{array}{llllllllllllllllllll}V & A & V & L & L & M & I & L & Y & L & V & S & V & G & S & S & S & S & E & R\end{array}$ GGT AAA GAG CCT CCA GGA CCG AAG CCT CTG CCT CTG CTT GGC AAC CTG CTG CAA CTT GAT 205 $\begin{array}{llllllllllllllllllll}G & K & E & P & P & G & P & K & P & L & P & L & L & G & N & L & L & Q & I & D\end{array}$ CTC CAG AGA CCC TAC AAA ACC CTG TGT CAG CTT TCC AAG AAA TAT GGA TCT GTA TTC ACA 265 $\begin{array}{llllllllllllllllllll}L & Q & R & P & Y & K & T & L & C & Q & L & S & K & K & Y & G & S & V & F & T\end{array}$ GTT TAT TTT GGG CCA AAA AAA GTG GTT GTC CTG TCT GGG TAC AGG ACC GTT AAA GAG GCT 325 $\begin{array}{lllllllllllllllllllll}V & Y & F & G & P & K & K & V & V & V & L & S & G & Y & R & T & V & K & E & A\end{array}$ TTA GTC AGA TAC GCT GAT GAA TTT GGA GAA AGA GAG GTT TCA CCT ATA TTT GAT GAT CTA 385 $\begin{array}{lllllllllllllllllllll}L & V & R & Y & A & D & E & F & G & E & R & E & V & S & P & I & F & D & D & L\end{array}$ AAC AAT GGT CAT GGA ATT TTG TTT TCA AAT GGA GAA ACA TGG AAA GAG ATG CGT CGC TTC 445 $\begin{array}{llllllllllllllllllll}N & N & G & H & G & I & L & F & S & N & G & E & T & W & K & E & M & R & R & F\end{array}$ GCC CTG ACC GCC CTC AGA GAT TTT GGG ATG GGG AAG CGA GTT GCT GAG GAG AAA ATC TTA 505 $\begin{array}{lllllllllllllllllllll}A & L & T & A & L & R & D & F & G & M & G & K & R & V & A & E & E & K & I & L\end{array}$ GAG GAG TGT GGA CAT CTC ATT CAA ACG ATT GAG AAT TAT AAA GGG GAA CCG TTT AAC ACG 565 $\begin{array}{llllllllllllllllllll}E & E & C & G & H & L & I & Q & T & I & E & N & Y & K & G & E & P & F & N & T\end{array}$ TCA CTT CCA CTG AAT TAT GCA ACA TCC AAC ATC ATC TCC TCT ATT GTG TAT GGA AGC AGA 625 $\begin{array}{llllllllllllllllllll}S & I & P & L & N & Y & A & T & S & N & I & I & S & S & I & V & Y & G & S & R\end{array}$ TTT GAA TAT GAA GAC CCT CGA TTT AGA AAC CTG GTT AGT CGA GCC AAT GAG AAC ATA AGC $\begin{array}{lllllllllllllllllllll}F & E & Y & E & D & P & R & F & R & N & L & V & S & R & A & N & E & N & I & S\end{array}$ TTA GCG GGC TCA GCA GAA ATC CAG CTC TAC AAT ATG TTT CCC CGG CTG GTC AGA TGG ATA 745 $\begin{array}{lllllllllllllllllllll}L & A & G & S & A & E & I & Q & L & Y & N & M & F & P & R & L & V & R & W & I\end{array}$ AAA AAA CGT CAT GTG ATT CTA GAA AAT GCT AAA ATG ACA GTC AGC AAT GTG AAA GAT TTA 805 $\begin{array}{llllllllllllllllllll}K & K & R & H & V & I & L & E & N & A & K & M & T & V & S & N & V & K & D & I\end{array}$ ATC CAC AAA CTG AAG GAG ACG CTG AAC CCT CAG ACA TGC AGA GGA CTG GTG GAC TGT TTT 865 $\begin{array}{llllllllllllllllllll}I & H & K & L & K & E & T & L & N & P & Q & T & C & R & G & L & V & D & C & F\end{array}$ CTG ATT CGG AAG CAG AAA GAA GAG GAC TCT TGT GTA AAA GAC ACT CAG TTC ACA GAG GAG 925 $\begin{array}{llllllllllllllllllll}L & I & R & K & Q & K & E & E & D & S & C & V & K & D & T & Q & F & T & E & E\end{array}$ AAC CTG ATA TTT ACA GTA TCC AAT CTG TTT TCT GCT GGA ACT GAC ACC ACA GCT GCA ACA 985 $\begin{array}{lllllllllllllllllllllll}N & I & I & F & T & V & S & N & L & F & S & A & G & T & D & T & T & A & A & T\end{array}$ TTG AGG TGG GGT TTG CTG CTC ATG GCC AAA TAT CCA CAA ATT CAG GAC CTa GTG CAA GAG $\begin{array}{llllllllllllllllllll}I & R & W & G & L & L & L & M & A & K & Y & P & Q & I & Q & D & L & V & Q & E\end{array}$ GAG CTG GCC AGG GTG GTC GGA GGC CGT GAA GTC CAA GTG GAA GAC CGT AAA AAC CTG CCA 1105 $\begin{array}{llllllllllllllllllll}E & I & A & R & V & V & G & G & R & E & V & Q & V & E & D & R & K & N & L & P\end{array}$ TAC ACT GAT GCT GTC ATC CAC GAG ATA CAG AGG CTG GCT AAC ATT GTC CCC ATG GCC GTA 1165 $\begin{array}{llllllllllllllllllll}Y & T & D & A & V & I & H & E & I & Q & R & L & A & N & I & V & P & M & A & V\end{array}$ CCA CAC AAA ACC AGT CGA GAC GTC ACC TTT CAG GGA TAC TTC ATT AAA GAG GGA ACA ACT $\begin{array}{llllllllllllllllllll}\mathrm{P} & \mathrm{H} & \mathrm{K} & \mathrm{T} & \mathrm{S} & \mathrm{R} & \mathrm{D} & \mathrm{V} & \mathrm{T} & \mathrm{F} & \mathrm{Q} & \mathrm{G} & \mathrm{Y} & \mathrm{F} & \mathrm{I} & \mathrm{K} & \mathrm{E} & \mathrm{G} & \mathrm{T} & \mathrm{T}\end{array}$ GTG TTT CCC CTG CTT ACG TCT GTT CTG AAT GAT GAA AGT GAA TGG GAA AGC CCT CAC TCC 1285 $\begin{array}{llllllllllllllllllll}V & F & P & L & L & T & S & V & L & N & D & E & S & E & W & E & S & P & H & S\end{array}$ TTC AAC CCT TCT CAT TTC TTG AAC AAG GAG GGC AAA TTC ATC AAG AGA GAT GCT TTC TTA 1345 $\begin{array}{lllllllllllllllllllll}F & N & P & S & H & F & L & N & K & E & G & K & F & I & K & R & D & A & F & I\end{array}$ CCA TTP TCT GCA GGT CGC AGG GTG TGT CTC GGG GAG GGT CTG GCT AAG ATG GAG CTT TTC 1405

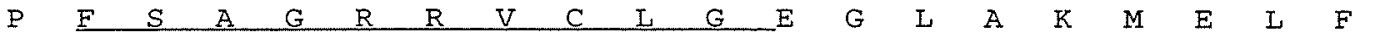
CTC TTG TTC AGC TCC CTG CTT CAG CGT TTT CGT TTC AAG CCT CCA CCG GGA GTT ACT GAA 1465 $\begin{array}{llllllllllllllllllll}L & L & F & S & S & I & L & Q & R & F & R & F & K & P & P & P & G & V & T & E\end{array}$ GAT GAG CTG GAT TTG ACT CCA GCG GTG GGC TTC ACC ATC CCA CCT TCT CCT CAT AAG CTC 1525 $\begin{array}{llllllllllllllllllll}D & E & L & D & L & T & P & A & V & G & F & T & I & P & P & S & P & H & K & L\end{array}$ TGT GCC ATC AGT CGG CAG taa ata agg aag cat aac att gtt cct ctg tgt atc agt gge 1585 $C$ A I $S$ R Q ctaatgtgctctactaaatgtaaaccttaattttatgtctcattgttcaaacagcttgacagagattgacatttttagt 1664 gcacactccttcacacctcgccactgttgcttttcttaagccgtgtaactacaaaaagtaagtcttactaaaacatga 1743 attgtttcgaaactactaaagactttagaaatgaaataatagtccagctgtacacaatgcatattaaataaatgtttc 1822 ccttcaaaaaaaaaaaaaaaaaaaa 
Figure 2. Nucletoide and predicted amino acid sequence for killifish CYP2N1. The same features are highlighted as described in the legend to Figure 1. 

$\begin{array}{llllllllllllllllllll}M & W & L & Y & N & F & L & L & V & L & D & L & K & A & I & L & I & F & I & F\end{array}$ TCC TTC CTT TTG ATC GCA GAC TTC CTT AGG AAC AGG AAA CCG GCA AAC TTT CCT CCA GGA 189 $\begin{array}{llllllllllllllllllll}S & F & L & L & I & A & D & F & L & R & N & R & K & P & A & N & F & P & P & G\end{array}$ CCA AAG GCT CTC CCC TTT GTG GGG AAC ATG TTA AAT CTG GAC AGC CAA CAT CCA CAC ATC 249 $\begin{array}{llllllllllllllllllll}P & K & A & I & P & F & V & G & N & M & L & N & L & D & S & Q & H & P & H & I\end{array}$ TTT TTC AGC AAG TTG GCT GAC ATC TAC GGG AAC GTC TTC AGC TTT CGC CTG GGC AAG GAA 309 $\begin{array}{lllllllllllllllllllll}F & F & S & K & L & A & D & I & Y & G & N & V & F & S & F & R & I & G & K & E\end{array}$ TCC ATG GTG GTC GTG AGC GGA CAC AAG ITG GTG AAA GAG GCC ATA GTG ACA CAA GGC GAG $\begin{array}{llllllllllllllllllll}S & M & V & V & V & S & G & H & K & L & V & K & E & A & I & V & T & Q & G & E\end{array}$ AAC TTT GTG GAT CGA CCA CCT AAT GCA ATC GCT GAA AGG TTT TAC ACT GAA CCC TCA GGT $\begin{array}{llllllllllllllllllll}N & F & V & D & R & P & P & N & A & I & A & E & R & F & Y & T & E & P & S & G\end{array}$ GGT CTT TTT TTT AAC AAC GGA GAG ATA TGG AAA AGG CAG CGG CGT TTT GCT TTA TCT ACC $\begin{array}{llllllllllllllllllll}G & L & F & F & N & N & G & E & I & W & K & R & Q & R & R & F & A & L & S & T\end{array}$ TTA CGC ACC TTT GGC CTG GGC AAA AAC ACG CTG GAG CTG AGC ATC TGT GAG GAG ATC CGA $\begin{array}{llllllllllllllllllll}L & R & T & F & G & L & G & K & N & T & L & E & L & S & I & C & E & E & I & R\end{array}$ CAT CTG CAG GAG GAA ATA GAG AAT GAG AAA GGT AAA CCC TTC AGC CCA GCA GGC CTC TTC $\begin{array}{llllllllllllllllllll}H & L & Q & E & E & I & E & N & E & K & G & K & P & F & S & P & A & G & I & F\end{array}$ AAC AAC GCT GTC TCC AAC ATA ATT TGC CAG CTG GTT ATG GGG AGA AGA TTT GAC TAC CAT $\begin{array}{lllllllllllllllllllll}N & N & A & V & S & N & I & I & C & Q & L & V & M & G & R & R & F & D & Y & H\end{array}$ GAC CAA AGC TTT CAG ACC ATG CTG AAG TAC ATG TCT GAA GCG CTT TGG CTG GAA GGG AGC $\begin{array}{llllllllllllllllllll}D & Q & S & F & Q & T & M & I & K & Y & M & S & E & A & L & W & L & E & G & S\end{array}$ ATA TGG GGT CAG CTT TAT CAG GCC TTT CCT CAA GTC ATG AAA TAT ATA CCG GGT CCT CAC

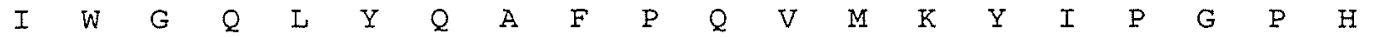
AAC AAA CTC TTC AGC AAT TTT ACT GCC ATA AAG GAG CTC CTG CAA GAA GAA ATA GAA AAG $\begin{array}{llllllllllllllllllll}\mathrm{N} & \mathrm{K} & \mathrm{L} & \mathrm{F} & \mathrm{S} & \mathrm{N} & \mathrm{F} & \mathrm{T} & \mathrm{A} & \mathrm{I} & \mathrm{K} & \mathrm{E} & \mathrm{L} & \mathrm{L} & \mathrm{Q} & \mathrm{E} & \mathrm{E} & \mathrm{I} & \mathrm{E} & \mathrm{K}\end{array}$ CAC AAG AAG GAT CTG GAT CAC AGC AAT CCC AGA GAC TAC ATT GAC ACC TTC CTC ATT AAA $\begin{array}{llllllllllllllllllll}H & K & K & D & L & D & H & S & N & P & R & D & Y & I & D & T & F & I & I & K\end{array}$ ATG GAG AAC CAA CAA GAA GCT GAG CTG GGA TTC ACA GAA CGT AAT CTG GCT TTC TGC TCT

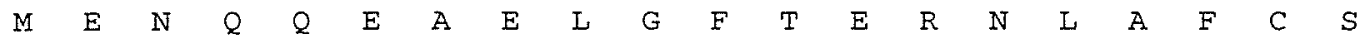
CTC GAC CTC TTC CTT GCT GGA ACA GAA ACA ACA GCC ACC ACA CTG CTG TGG GCC TTG CTT $\begin{array}{llllllllllllllllllll}L & D & L & F & L & A & G & T & E & T & T & A & T & T & L & L & W & A & L & L\end{array}$ TTC CTC ATA AAA TAC CCT GAA GTC CAA GAG AAA GTC CAT GCA GAG ATC GAC AGA GTT ATT $\begin{array}{llllllllllllllllllll} & \mathrm{L} & I & K & \mathrm{Y} & \mathrm{P} & \mathrm{E} & \mathrm{V} & \mathrm{Q} & \mathrm{E} & \mathrm{K} & \mathrm{V} & \mathrm{H} & \mathrm{A} & \mathrm{E} & \mathrm{I} & \mathrm{D} & \mathrm{R} & \mathrm{V} & \mathrm{I}\end{array}$ GGA CAG ACT CGC CTG CCC TCC ATG GCC GAC AGG CCG AAC CTG CCT TAC ACT GAT GCT GTG 1149 $\begin{array}{lllllllllllllllllllll}G & Q & T & R & L & P & S & M & A & D & R & P & N & L & P & Y & T & D & A & V\end{array}$ ATC CAT GAG ATC CAG AGG ATG TCA AAC ATT GTT CCT CTG AAT GGA CTC AGG GTT GCT TCC $\begin{array}{lllllllllllllllllllll}I & H & E & I & \& & R & M & S & N & I & V & P & L & N & G & L & R & V & A & S\end{array}$ AAG GAT ACA ACT CTG GGT GGT TAC TTC ATA CCA AAG GGA ACA GCG GTG ATG CCC ATG CTG $\begin{array}{llllllllllllllllllll}K & D & T & T & L & G & G & Y & F & I & P & K & G & T & A & V & M & P & M & L\end{array}$ ACC TCT GTA CTG TTT GAC AAA ACT GAG TGG GAG ACT CCA GAC ACC TTC AAT CCT GGA CAC $\begin{array}{llllllllllllllllllll}T & S & V & I & F & D & K & T & E & W & E & T & P & D & T & F & N & P & G & H\end{array}$ TTC CTG GAT GCT AAT GGG AAA TTT GTG AAG AAA GAA GCG TTC CTG CCC TTC TCT GCA GGG $\begin{array}{llllllllllllllllllll}F & L & D & A & N & G & K & F & V & K & K & E & A & F & L & P & F & S & A & G\end{array}$ AAA CGT GTG TGC CTC GGT GAA GGC CTC GCP AAG ATG GAG CTG TTC CTG TTT CTG GTC GCC $\begin{array}{lllllllllllllllllllll}K & R & V & C & L & G & E & G & L & A & K & M & E & I & F & L & F & L & V & A\end{array}$ TTG CTG CAG AAG TTC TCT TTC TCT GCT CCP GAA GGA GTT GAA CTG AGC ACT GAA GGA ATA 1509 $\begin{array}{llllllllllllllllllll}L & L & \varrho & K & F & S & F & S & A & P & E & G & V & E & L & S & T & E & G & I\end{array}$ ACG GGG ATT ACC CTT GTG CCT CAC CCC TAC AAG GTC TCT GCT AAG GCT CGC tga gCt gtg

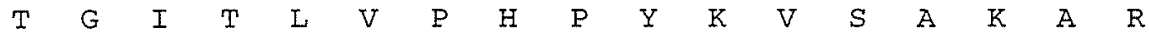
gacatctaaaaaaaacaaaaacaagtttggctcaaaagaagaccaggccactgttgtttgattgcagcttgttaagaaa acatgttgattgctgatagatgtgtgcatttattctgagcgatcatcgctaaaaacacaaaatacttttgcattaatga ctcctaacaggattgagcaaggattctgctttgcaacatcctccctaaaataagtccaaagctgaaagcaagtttaa taaaaatatttgctccattttattaaaaggactaaagagccaaatactaaaacctggagatgtatctatttggaaaga tatggctatctactttattgtaaacacagtgttcgaacaggtaagttgtaaatttgacaaggggtattattattatta ttattattattaatcttcttttatacaagttagtcccttgagactcagcgcctcattgtataagagaaacttgtttaaa cacataacataaattacaacaatttacaacagcagttataaatttgtcccaaaaaaaggtttaaatttcatgacaa aattaaaatatgcacaaatgaaaaaaaaa 
Figure 3. Nucletoide and predicted amino acid sequence for killifish CYP2N2. The same features are highlighted as described in the legend to Figure 1. 
ttgactgaactgggcctgcttgacacaaagcaattcaattattcttaaacagacctttta gaagctgatgctccacaccgggagggcagtttggactactacaggcagttcttccattgcagttctgctcgcattaacc ATG TGG TTT TAT AAT CTT TTG CTG AGC CTC GAC GTG AAG GGG CTT TTC TTG TTT ATT TTT $\begin{array}{llllllllllllllllllll}M & W & F & Y & N & I & L & L & S & L & D & V & K & G & L & F & L & F & I & F\end{array}$ CTT TTC CTT CTG ATA GCG GAC TTC TAC AAA AGC AGA AAA CCA GCA AAT TTT CCT CCA GGA 259 $\begin{array}{lllllllllllllllllllllll}L & F & I & L & I & A & D & F & Y & K & S & R & K & P & A & N & F & P & P & G\end{array}$ CCA AAG GCT CTT CCC TTT GTG GGG AAT TTC TTC AGT TTG GAC AGC AAA CAT CCA CAC GTC $\begin{array}{llllllllllllllllllll}P & K & A & L & P & F & V & G & N & F & F & S & L & D & S & K & H & P & H & V\end{array}$ TAT TTC CAA AAG CTA GCT GAG ATC TAT GGG AAC GTC TTC AGC TTC CGC CTC GGC CGG GAC $\begin{array}{llllllllllllllllllll}Y & F & Q & K & I & A & E & I & Y & G & N & V & F & S & F & R & I & G & R & D\end{array}$ TCG ATC GTG TTT CTG AAC GGA TAC AAG GCG GTG AGA GAG GCG TTA GTG ACC CAA GCT GAG $\begin{array}{lllllllllllllllllllll}S & I & V & F & L & N & G & Y & K & A & V & R & E & A & L & V & T & Q & A & E\end{array}$ AAC TTT GTG GAC CGA CCA TTC AAC GCC ATA ACA GAC AGG TTT TAC ACT GAA CCC TCA GCT $\begin{array}{lllllllllllllllllllll}N & F & V & D & R & P & F & N & A & I & T & D & R & F & Y & T & E & P & S & A\end{array}$ GGT ATC TTT ATG AGC AAT GGT GAA AAA TGG AAG AAA CAG CGA CGT TTT GCT TTA TCT ACC $\begin{array}{llllllllllllllllllll}G & I & F & M & S & N & G & E & K & W & K & K & Q & R & R & F & A & L & S & T\end{array}$ CTA CGC AAC TTT GGC CTG GGC AAA AAC TCT TTG GAG CAG AGT GTT TCT GAG GAG ATC CAA $\begin{array}{llllllllllllllllllll}I & R & N & F & G & L & G & K & N & S & L & E & Q & S & V & S & E & E & I & Q\end{array}$ CAC CTG CAG GAG GAA ATG GAG ATC GAA AAA GGT AAA CCT TTC AAC CCA TCA GGC CTT TTT $\begin{array}{llllllllllllllllllll}H & L & Q & E & E & M & E & I & E & K & G & K & P & F & N & P & S & G & L & F\end{array}$ ACC AAT GCC GTG TCC AAC ATC ATC TGC CAG CTG GTC ATG GGG AAA AGA TAC GAC TAC ACC $\begin{array}{llllllllllllllllllll}T & N & A & V & S & N & I & I & C & Q & I & V & M & G & K & R & Y & D & Y & T\end{array}$ GAC CAC AGG TTT CAG ATG ATG CTC AGG TGC ATG TCT GAG GCG GTT TTG CTG GAG GGG AAC $\begin{array}{llllllllllllllllllll}D & H & R & F & Q & M & M & L & R & C & M & S & E & A & V & L & L & E & G & N\end{array}$ GTT TGG GGC CAA CTC TAC ATG GCG TTT CCG AGT GTG ATG AGG TAC ATG CCA GGT CCT CAC $\begin{array}{lllllllllllllllllllll}V & W & G & Q & L & Y & M & A & F & P & S & V & M & R & Y & M & P & G & P & H\end{array}$ AAC AAA ATC TTC AGC CAC TTC TCC AGT GTA GAA CAA TTC CTT TAT GAG GAA GTA GAG CAG $\begin{array}{llllllllllllllllllll}N & K & I & F & S & H & F & S & S & V & E & Q & F & L & Y & E & E & V & E & Q\end{array}$ CAC AAG AAG GAT CTG GAT CGC GAC AAT CCC AGA GAC TAC ATT GAC ACT TTC CTC ATT GAG $\begin{array}{llllllllllllllllllll}H & \mathrm{~K} & \mathrm{~K} & \mathrm{D} & \mathrm{L} & \mathrm{D} & \mathrm{R} & \mathrm{D} & \mathrm{N} & \mathrm{P} & \mathrm{R} & \mathrm{D} & \mathrm{Y} & \mathrm{I} & \mathrm{D} & \mathrm{T} & \mathrm{F} & \mathrm{L} & \mathrm{I} & \mathrm{E}\end{array}$ ATG GAG AAC CAC AAA GAG TCT GAC CTT GGC TTC ACG GAG GCC AAT CTG GTT TAC TGC GCC $\begin{array}{llllllllllllllllllll}M & E & N & H & K & E & S & D & L & G & F & T & E & A & N & L & V & Y & C & A\end{array}$ ATT GAC CTT TTC CTC GCT GGA ACA GAA ACG ACA GCC ACC ACA CTG CTG TGG GCT TTG GTT $\begin{array}{lllllllllllllllllllll}I & D & I & F & L & A & G & T & E & T & T & A & T & T & I & I & W & A & L & V\end{array}$ TTC CTC GTA AAA TAT CCT GAG GTT CAA GAG AAA GTC CAG GCA GAG ATC GAC TCA GTG ATC $\begin{array}{lllllllllllllllllllll}F & L & V & K & Y & P & E & V & Q & E & K & V & Q & A & E & I & D & S & V & I\end{array}$ GAA CAG GCC CGC CTG CCC TCC ATG GCC GAC AGA TCT AGC ATG CCT TAC ACC GAC GCT GTG $\begin{array}{llllllllllllllllllll}E & Q & A & R & L & P & S & M & A & D & R & S & S & M & P & Y & T & D & A & V\end{array}$ ATC CAC GAG ATC CAG AGA ATA GGA AAT ATA CTT CCT CTC AAT GGA ATG AGG GTT GCT GCC

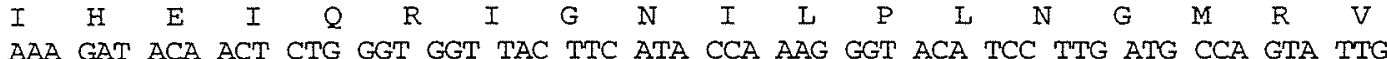
$\begin{array}{lllllllllllllllllllll}K & D & T & T & I & G & G & Y & F & I & P & K & G & T & S & I & M & P & V & I\end{array}$ ACC TCT GTA CTG TTC GAC AAA GCT GAA TGG GCT TGC CCA GAC ACC TTC AAT CCT GGA CAC $\begin{array}{llllllllllllllllllll}T & S & V & L & F & D & K & A & E & W & A & C & P & D & T & F & N & P & G & H\end{array}$ TTC CTG GAT GAC AAT GGG AAG TTT GTG AAG AGA GAC GCC TTC CTC CCC TTC TCT GCG GGA $\begin{array}{llllllllllllllllllll}F & L & D & D & N & G & K & F & V & K & R & D & A & F & L & P & F & S & A & G\end{array}$ AAA CGG GCT TGC ATT GGC GAA AGC CTC GCT AAG ATG GAG CTG TTC CTG TTT TTG GTC GCC $\begin{array}{lllllllllllllllllllll}K & R & A & C & I & G & E & S & L & A & K & M & E & L & F & L & F & L & V & A\end{array}$ TTG CTG CAG AAG TTC ACG TTC TCT GTT CCT GAG GGA GTT GAA CTG AGC ACT GAA GGA ATC $\begin{array}{llllllllllllllllllll}I & L & Q & K & F & T & F & S & V & P & E & G & V & E & L & S & T & E & G & I\end{array}$ ACA GGA ACG ACA CGA GTG CCT CAC CCC TAC AAA GTG TCG GCT AAG ATT CGC tga gCt gCg $\begin{array}{lllllllllllllllllll}T & G & T & T & R & V & P & H & P & Y & K & V & S & A & K & I & R\end{array}$ gacgtctcaccaaagtgttttctactcaaaaagattgagcatattgttttgttttgtaaaaactgttgcattattgctg cctattattattacactatactgattgtctttagctgtatacatcattaaggctgaaatgtatattaaccaaaagattt tactgtgttaaacaattgtcaaaatacctctaaaatcggtggttggtcggtgtggtgagctcgaccatattccctgtta ctcacaaattaagacataatttggacattaaatgtattacgtaaaatagataacataagacctctgtaatggtatgttt taattgatcatttctcttactgaattatatggttgtctttcttttttaaaggatcaagcacttatgtattaaataa tgaaataatgattgttatagtgtcttaacactcttattttctcttaagactgttatagacagtcttaagagaaaataga cttgaacatcagtctgcccttctaaatttaaagcttctcctgaacgtattaataagcagccttagattatatatatat atataaatgtgtgtatatctggagcatgtaacaaaagtaatttccctctaggattattaaagtatgtctgattctgatt 
ctgagataggaagttgattaaattttaatttgtaatttgttaaattgcttaactccctgccaaagcatactaattaaag

2350

aagcatatcaaattttaaaaaaaaa 
Figure 4. Nucletoide and predicted amino acid sequence for killifish CYP2P2. The same features are highlighted as described in the legend to Figure 1. 
ggaattcggcacgaggcggaggcagtcttggggaa 35 ATG GAA GCT TTA TAC AGT CTT CTC GGC TTG GAG TGG TTA GAC ACC AGG AGT GTT TTA ATA 95

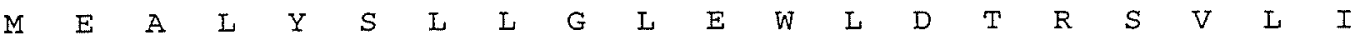
TTT TTC TGT GTG TUT TTG CTG CTG AGC GAT ATT TTG AAG AAC AGA AAA CCA AAA AAC TTT 155 $\begin{array}{llllllllllllllllllll}F & F & C & V & F & L & L & L & S & D & I & L & K & N & R & K & P & K & N & F\end{array}$ CCT CCT GGA CCC GCA GCT CTT CCT TTT ATT GGA GAT CTT CAT CAC ATC AAC CCA TCG AGA 215 $\begin{array}{lllllllllllllllllllllll}P & P & G & P & A & A & L & P & F & I & G & D & L & H & H & I & N & P & S & R\end{array}$ ATT CAC CTG CAG ATC ACC GAT TTT GCA GAG AAA TAT GGC AAT GTT TTC AGT CTT CAT CTC 275

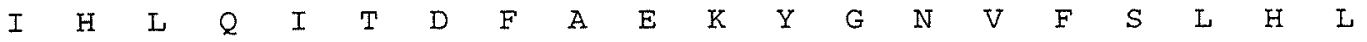
TTT GGA GGA AAA GCT GTG GTC ATT AAT GGA TAC AAA CAC GTG AAA GAG GCT CTT GTT GAA 335 $\begin{array}{llllllllllllllllllll}F & G & G & K & A & V & V & I & N & G & Y & K & H & V & K & E & A & L & V & E\end{array}$ AAA GGA GAA GAC TTC ATG GAT CGG CCC ACC ATT CCA TTG TTT TCT GAT GTT TTC AAG AAC 395 $\begin{array}{llllllllllllllllllll}K & G & E & D & F & M & D & R & P & T & I & P & I & F & S & D & V & F & K & N\end{array}$ AAA GGT ATT GTG ATG TCC AAC GGT TAC CCA TGG AAG GTA CAG AGG AGG TTT GCT CTT CAT 455 $\begin{array}{llllllllllllllllllll}K & G & I & V & M & S & N & G & Y & P & W & K & V & Q & R & R & F & A & L & H\end{array}$ GCG CTG AGA AAC TTT GGT CTG GGA AAG AAG ACC ATG GAG AGA TAT ATC CAG CAG GAG TGC 515 $\begin{array}{lllllllllllllllllllll}A & L & R & N & F & G & I & G & K & K & T & M & E & R & Y & I & Q & Q & E & C\end{array}$ CAG TAT CTC AAT GAA GTT TTT GTT GAT CAG CAA GGC AAG CCA TTT TCT GGT CAG ACA CTG $\begin{array}{llllllllllllllllllll}Q & Y & L & N & E & V & F & V & D & Q & Q & G & K & P & F & S & G & Q & T & L\end{array}$ ATA AAC AAT GCT GTG TCC AAC ATC ATC TGC TGT TTG GTC TTT GGG AAC CGA TTT GAG TAC $\begin{array}{llllllllllllllllllll}I & N & N & A & V & S & N & I & I & C & C & I & V & F & G & N & R & F & E & Y\end{array}$ GAC GAC AAG GAG TAC CAC ACT ATT CTT GAT AAC ATG AAT GAG CTT TTG CGC CTA CAA GGA $\begin{array}{llllllllllllllllllll}D & D & K & E & Y & H & T & I & I & D & N & M & N & E & I & I & R & L & Q & G\end{array}$ GGT TTT TGG GTG CAG GTA TAT AAC ATG TTT CCC TCT GTG ATG AAG TGG CTG CCA GGA CCT 755 $\begin{array}{llllllllllllllllllll}G & F & W & V & Q & V & Y & N & M & F & P & S & V & M & K & W & L & P & G & P\end{array}$ CAT AAG AAA ATA TTC ATC CAT CTA CAG AAG ATA ATT GAC TTT TTA GAA ATT CGG ATC AAA 815

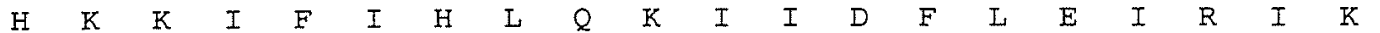
GAA CAC AGA GAG AAC CTT GAC CCC TCA TCT CCA AGA GAC TAC ATC GAC TCC TTT CTG ATA $\begin{array}{llllllllllllllllllll}E & H & R & E & N & L & D & P & S & S & P & R & D & Y & I & D & S & F & L & I\end{array}$ GAA ATG GGA GAT AAA GAG GAC AAA GAT TCT GGT TTT GAT CTC TTC AAC TTG TCA GCT TGC $\begin{array}{llllllllllllllllllll}E & M & G & D & K & E & D & K & D & S & G & F & D & L & F & N & L & S & A & C\end{array}$ ACT CTG GAC CTG TTT GCT GCT GGA ACT GAA ACC ACC ACC ACT ACT TTG CAC TGG GGG CTG

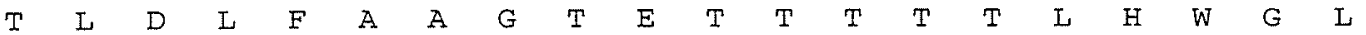
TTG TAC ATG ATT TAC TAC CCT GAC ATA CAA GAG CGT GTC CAT GCT GAG ATC AAT GCT GTG $\begin{array}{llllllllllllllllllll}L & Y & M & I & Y & Y & P & D & I & Q & E & R & V & H & A & E & I & N & A & V\end{array}$ ATT GGT TCA TCC AGA CAA CCT GCT GTG GCT GAC AGG GAA AAC ATG CCC TAC ACT GAT GCA 1115 $\begin{array}{llllllllllllllllllll}I & G & S & S & R & Q & P & A & V & A & D & R & E & N & M & P & Y & T & D & A\end{array}$ GTC ATC CAT GAG ATA CAA AGG ATG GGA AAC ATC CTT CCT CTC AAT GTG GCC CGC ATG ACA $\begin{array}{llllllllllllllllllll}V & I & H & E & I & Q & R & M & G & N & I & L & P & L & N & V & A & R & M & T\end{array}$ AGC AAA GAC ACC ACC CTG GAT AAA TAC TCA ATT CCA AAG GGg ACT GTG ATC ATA GCA ACT $\begin{array}{llllllllllllllllllll}S & K & D & T & T & L & D & K & Y & S & I & P & K & G & T & V & I & I & A & T\end{array}$ CTT CAC TCT GTC CTC CAT GAT GAG TCC ATG TGG GAG ACT CCA CAC TCC TEC AAC CCT CAG 1295 $\begin{array}{llllllllllllllllllll}\mathrm{L} & \mathrm{H} & \mathrm{S} & \mathrm{V} & \mathrm{I} & \mathrm{H} & \mathrm{D} & \mathrm{E} & \mathrm{S} & \mathrm{M} & \mathrm{W} & \mathrm{E} & \mathrm{T} & \mathrm{P} & \mathrm{H} & \mathrm{S} & \mathrm{F} & \mathrm{N} & \mathrm{P} & Q\end{array}$ CAC TTT CTG GAC CAG GAT GGT AaA TTT AGG AAA AGA GAC GCC TTT ATG CCT TTT TCT GCA 1355 $\begin{array}{llllllllllllllllllll}H & F & L & D & Q & D & G & K & F & R & K & R & D & A & F & M & P & F & S & A\end{array}$ GGT AAA CGT GTG TGT CTT GGA GAG CAG CTG GCC AGG ATG GAG TTA TTC CTC TTC TTC ACC 1415 $\begin{array}{llllllllllllllllllll}G & K & R & V & C & L & G & E & Q & I & A & R & M & E & L & F & I & F & F & T\end{array}$ TCC CTC CTT CAG AGG TTT AAA TTC TCC CCT CCT CCA GGA GAG CAG CCT AGT CTG GAG TAC 1475 $\begin{array}{llllllllllllllllllll}S & L & L & Q & R & F & K & F & S & P & P & P & G & E & Q & P & S & L & E & Y\end{array}$ AAA CTG GGA GCC ACT CAT TGC CCC AAa CCT TAC CGC CTC TGT GCC GTA CCA CGT taa aCC 1535 $\begin{array}{llllllllllllllllll}K & L & G & A & T & H & C & P & K & P & Y & R & L & C & A & V & P & R\end{array}$ aaaagaagtgttgacagcgccactgtgcatcccttctgctgtgtttgatcccagcggcgcctaacttggcggtattaaa gcagttaaggtatcaaaactttggtctgatactttggtgaaagttagactataaccacacaaaattgtaatattattat cctcggtttttggttaattcccagctcctgtcattctcagcaattgcttccacttgtcaataaatcagtttaactgca tagcattgttttgggctgaattccgggttcaccttcaaaccagatcatcacaaaaatgaaataactgtaaagacttttg ggataaatgtgtagaaatatagttgtataaataatttatgctggaaaggaacagtgtaaatcaaatggtcatattttaa tctaaaatacttgccatcctgatgtggaaaattatgttttttctaaatctgcggcaaactctgtaagacttaattaca aacctgctgatataatttctgcacctgctgatgggaattagataaaatgaaatattcctttgaaacccactttgtat atgaccaacttcattaaaaacatgcagataancgtaaaaaaaaaaaaaaa 
ccacttgtttaatgaaaatatataacagcatattgaatgtaatcaaacatatgtgagaacaca attggagaatattatttacatatcaaaattacagactaaggtgttgtgttaaataaaaatattttttatctgttatgc aaggacacacatcaaatgagggatttccctgaggttgttcaagaaccaacatggaagtctgatgagttaaatcagagac ATG GAG GCA ATA CGC AGT GTP CTC GGT TTA GAG TGG ATA GAC GGC AGG GGT GTT TTG GTA $\begin{array}{llllllllllllllllllll}M & E & A & I & R & S & V & L & G & I & E & W & I & D & G & R & G & V & I & V\end{array}$ TTT TTC TTC GTC TTT CTG CTG CTG AGC GAT GTT TTG AGG AAC CGA AAA CCA AAG AAC TTT 280 $\begin{array}{llllllllllllllllllll} & \mathrm{F} & \mathrm{F} & \mathrm{V} & \mathrm{F} & \mathrm{L} & \mathrm{L} & \mathrm{L} & \mathrm{S} & \mathrm{D} & \mathrm{V} & \mathrm{I} & \mathrm{R} & \mathrm{N} & \mathrm{R} & \mathrm{K} & \mathrm{P} & \mathrm{K} & \mathrm{N} & \mathrm{F}\end{array}$ CCT CCT GGA CCT CTG GCT CTA CCA TTT ATT AGA GAT CTT CAC CGC ATC AGA CCT GCA AGA 400

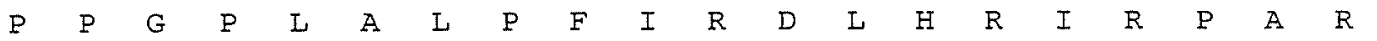
CTI CAC CTG CAG CTC ACA GAG TTT GCA GAA ACA TAT GGA GAC ATT TAC AGC CTC CAT CTC 460

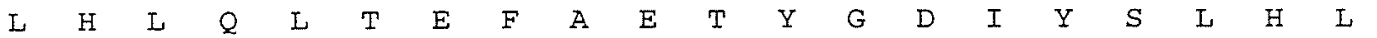
TTT GGT GGC AGA GCT GTG ATC ATA AAC GGC TAC AAA CAT GTG AAA GAG GCG CTG GTC CAA 520 $\begin{array}{llllllllllllllllllll} & G & G & R & A & V & I & I & N & G & Y & K & H & V & K & E & A & I & V & Q\end{array}$ AAA GGA GAA GAC TTC ATG GAT CGT CCC AAC ATT CCA CTT TTT GCT GAT TTT TTT AAT AAT $\begin{array}{llllllllllllllllllll}K & G & E & D & F & M & D & R & P & N & I & P & I & F & A & D & F & F & N & N\end{array}$ AAA GGT CTT GTG ATG TCA AAT GGT TAT CAA TGG AAG GTG CAG AGG AGG TTT GCT CTT CAT

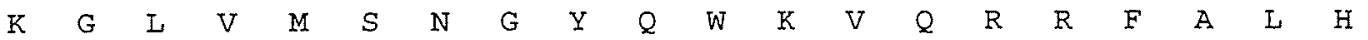
ACA CTC AGA CAC TCT GGT CTA GGA AAG AAG GCC ATG GAG CGA TAC ATC CAG CAA GAG TGC 7O0 $\begin{array}{lllllllllllllllllllll}T & \mathrm{~L} & \mathrm{R} & \mathrm{H} & \mathrm{S} & \mathrm{G} & \mathrm{I} & \mathrm{G} & \mathrm{K} & \mathrm{K} & \mathrm{A} & \mathrm{M} & \mathrm{E} & \mathrm{R} & \mathrm{Y} & \mathrm{I} & \mathrm{Q} & \mathrm{Q} & \mathrm{E} & \mathrm{C}\end{array}$ CAG TAT CTC AAT GAG GCT TTC TCA GAA CAG CAA GGC AAG CCT TTT AAT GGC CAG GCA CTG 760 $\begin{array}{lllllllllllllllllllll}Q & Y & L & N & E & A & F & S & E & Q & Q & G & K & P & F & N & G & Q & A & L\end{array}$ ATA AAC AAT GCT GTA TCT AAC ATC ATC TGC TGC TTG GTC TTP GGG AAT CGC TAC GAA TAC $\begin{array}{llllllllllllllllllll}I & N & N & A & V & S & N & I & I & C & C & L & V & F & G & N & R & Y & E & Y\end{array}$ AAT GAC AAA CAG TAC CAA ACT ATT CTT CAG TAC TTC AAT GAG GCA GTT CGC TTA CAG GGA $\begin{array}{llllllllllllllllllll}N & D & K & Q & Y & Q & T & I & I & Q & Y & F & N & E & A & V & R & L & Q & G\end{array}$ GAT TTG TCA GTT CAG ATT TAT AAC TCG ATC CCC GGA TTG ATG AGG TGG CTG CCT GGA TCA $\begin{array}{llllllllllllllllllllll}D & L & S & V & Q & I & Y & N & S & I & P & G & I & M & R & W & L & P & G & S\end{array}$ CAT AAA AAA ATA TTC ATG ATA CTA CAG AAG CTT GTT GAC TTT GTA GAA ATT CGG ATC AAA 1000 $\begin{array}{llllllllllllllllllll}H & K & K & I & F & M & I & L & Q & K & L & V & D & F & V & E & I & R & I & K\end{array}$ GAA CAC AGA GAG AAC CTT GAC CCC TCA TCT CCA AGA GAC TAC ATC GAC TCC TTC CTG ATA 1060 $\begin{array}{llllllllllllllllllll}E & H & R & E & N & I & D & P & S & S & P & R & D & Y & I & D & S & F & L & I\end{array}$ GAA ATG GGA GAG AAA GAA GAC AAA GAT TCT GGT TTT GAG CTC AGC AAT TTA TGT GCT TGC $\begin{array}{llllllllllllllllllllll}E & M & G & E & K & E & D & K & D & S & G & F & E & L & S & N & I & C & A & C\end{array}$ ACT CTG GAC CTG TWT GGT GCT GGA ACT GAA ACC ACC ACT ACC ACT TTA CAC TGG GGT CTG

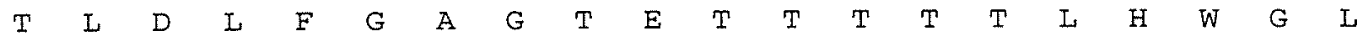
CTG TAC ATG ATC TAC TAT CCT CAG ATA CAA GAG CGT GTC CAG GCC GAA ATC GAT GCT GTG $\begin{array}{llllllllllllllllllll}I & Y & M & I & Y & Y & P & Q & I & Q & E & R & V & Q & A & E & I & D & A & V\end{array}$ ATT GGT CCG TCC AGG CAG CCC TCT GTG GCT GAC AGA GAA AAC ATG CCC TAC ACT GAT GCA 1300 $\begin{array}{lllllllllllllllllllll}I & G & P & S & R & Q & P & S & V & A & D & R & E & N & M & P & Y & T & D & A\end{array}$ GTA ATC CAT GAG ATA CAG AGG ATG GGT AAC ATC ATC CCC CTI AAC CTG CCT CGC ATG GCT 1360 $\begin{array}{lllllllllllllllllllll}V & I & H & E & I & Q & R & M & G & N & I & I & P & L & N & I & P & R & M & A\end{array}$ AAC AAA GAC ACC ACT CTG GAT AAA TAT TCA ATC CCA AAG GGA ACT ATT ATT ATA CCC ACA 1420

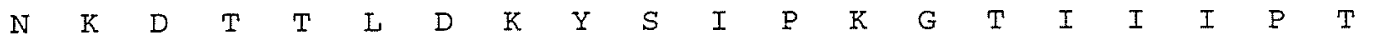
CTT CAC TCT GTC CTC CAA GAT AAG TCA ATA TGG GAG ACT CCA CAG ACC TTC AAT CCT CAA 1480 $\begin{array}{llllllllllllllllllll}\mathrm{L} & \mathrm{H} & \mathrm{S} & \mathrm{V} & \mathrm{I} & \mathrm{Q} & \mathrm{D} & \mathrm{K} & \mathrm{S} & \mathrm{I} & \mathrm{W} & \mathrm{E} & \mathrm{T} & \mathrm{P} & \mathrm{Q} & \mathrm{T} & \mathrm{F} & \mathrm{N} & \mathrm{P} & Q\end{array}$ CAC TTT CTG GAC CAG GAT GGT CAA TTT AGG AAG AGG GAC GCC TTC ATG CCT TTT TCT ACA 1540

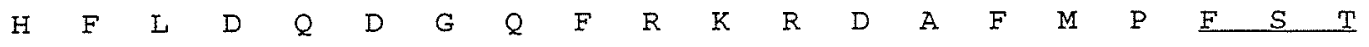
GGT AAG CGT GTG TGC CTC GGG GAG CAG CTG GCC AGG ATG GAG TTA TTC CTC TTC TTC ACC 1600 $\begin{array}{lllllllllllllllllllllll}\mathrm{G} & \mathrm{K} & \mathrm{R} & \mathrm{V} & \mathrm{C} & \mathrm{L} & \mathrm{G} & \mathrm{E} & \mathrm{Q} & \mathrm{L} & \mathrm{A} & \mathrm{R} & \mathrm{M} & \mathrm{E} & \mathrm{I} & \mathrm{F} & \mathrm{L} & \mathrm{F} & \mathrm{F} & \mathrm{T}\end{array}$ TCC CTC CTT CAG AGG TTP: :ACA TTC TCA GCT CCT GCT GGA GAG GAG CCC AGC CTG GAG TTC I660 $\begin{array}{lllllllllllllllllllll}S & L & L & Q & R & F & T & F & S & A & P & A & G & E & E & P & S & L & E & F\end{array}$ AAA CTG GGA GCA ACT CGC TCT CCC AAG CCT TAC CGC CTC TGC GCT ACA CCT CGT taa aCt 1720 $\begin{array}{llllllllllllllllll}K & L & G & A & T & R & S & P & K & P & Y & R & I & C & A & T & P & R\end{array}$ aagcacggtttcctctgttaatgtataaaccatggagagaaataaatgagaaaaatgcccctacagtcagttctctgaa cagtaaaatcaaactgtagtggtttatttttaagtatgtatgagtatgagatctcctttgtaaaataagtttgacag tcatgattatatacaataaagtacttatatttgttctgataaaaaaaaaaaaaaaaaaa

Figure 5. Nucletoide and predicted amino acid sequence for killifish CYP2P3. The same features are highlighted as described in the legend to Figure 1. 
ATG GAG ACA ATC CTG AAT GTT CTT GGC TTG GGG TGG ATA GAC AGC AGG AGC ATT TTG ATA 60 $\begin{array}{lllllllllllllllllllll}M & E & T & I & L & N & V & L & G & L & G & W & I & D & S & R & S & I & L & I\end{array}$ TTC CTG TTT GTT TTT CTG CTG CTG GCA GAT GTT TTG AAG AAC AGG GTG CCT AGA AAC TTT 120 $\begin{array}{llllllllllllllllllll}F & L & F & V & F & L & L & L & A & D & V & L & K & N & R & V & P & R & N & F\end{array}$ CCG CCG GGA CCG TGG TCT TTT CCT CTA GTT GGA GAT CTT CCT CGC ATT GAA GCT TCA AAA 180 $\begin{array}{llllllllllllllllllll}\mathrm{P} & \mathrm{P} & \mathrm{G} & \mathrm{P} & \mathrm{W} & \mathrm{S} & \mathrm{F} & \mathrm{P} & \mathrm{I} & \mathrm{V} & \mathrm{G} & \mathrm{D} & \mathrm{L} & \mathrm{P} & \mathrm{R} & \mathrm{I} & \mathrm{E} & \mathrm{A} & \mathrm{S} & \mathrm{K}\end{array}$

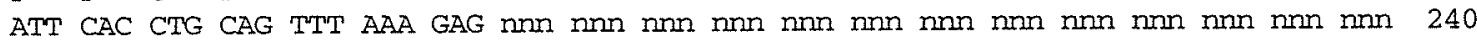
$\begin{array}{llllllllllllllllllll}I & H & L & Q & F & K & E & X & X & X & X & X & X & X & X & X & X & X & X & X\end{array}$ nnn GGG GGA AGG ATT GCC ATC ATT AAC AGC TAC AAG TTC ATG ACA GAG GCT CTG GTC CAA 300 $\begin{array}{lllllllllllllllllllll}X & G & G & R & I & A & I & I & N & S & Y & K & F & M & T & E & A & L & V & Q\end{array}$ AGA GGA GAG GAC TTC ACA GAT CGT CCC AGC ATC CCA CTG TTT GAA GAC GTA TTT GGG AAT 360 $\begin{array}{llllllllllllllllllll}R & G & E & D & F & T & D & R & P & S & I & P & L & F & E & D & V & F & G & N\end{array}$ AGA GGT CTT GTA GGT TCC AGT GGT TAT CCA TGG AAG CAG CAG AGG AGA TTT GCT CTT CAC 420

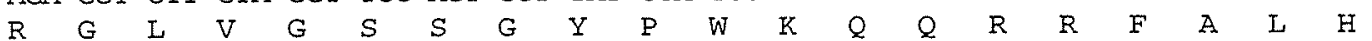
ACA CTC AGA AAC TTT GGT CTG GGA AAG AAA ACC TTG GAG CGA TCC ATC CAG CAG GAG TGT 480 $\begin{array}{llllllllllllllllllll}T & L & R & N & F & G & L & G & K & K & T & L & E & R & S & I & Q & Q & E & C\end{array}$ CAG TAT CTC ACA GAG GCT TTT GCT GAT CAA CAA GGT CAG CCC TTT AAT GCC CAG AAA CTG 580 $\begin{array}{llllllllllllllllllll}Q & Y & I & T & E & A & F & A & D & Q & Q & G & Q & P & F & N & A & Q & K & L\end{array}$ ATC AAC AAC GCT GTA TCC AAC ATC ATC TGC TGT TTG GTC TTT GGG AAC CGA TTT GAA TAC 600 $\begin{array}{llllllllllllllllllll}I & N & N & A & V & S & N & I & I & C & C & L & V & F & G & N & R & F & E & Y\end{array}$ AGT GAC AAG CAG TTC CAG ACC ATC CTG CAA CTG TTG AAC GAG ACA CTT TAC TTA GAA GGG 660 $\begin{array}{lllllllllllllllllllll}S & D & K & Q & F & Q & T & I & L & Q & L & L & N & E & T & L & Y & I & E & G\end{array}$ ACT GTT TGG GCA CAG ATG TAC AAC ACA ATG CCC TGG CTG ATG AGA TGG CTG CCT GGA CCT 720 $\begin{array}{llllllllllllllllllll}T & V & W & A & Q & M & Y & N & T & M & P & W & L & M & R & W & L & P & G & P\end{array}$ CAT CAG AGA ATA TTT TCC ATA ACA AAT GAG TTA CGT AGT TTT GTT AAA GTA AGG ATC AAC 780 $\begin{array}{llllllllllllllllllll}H & Q & R & I & F & S & I & T & N & E & L & R & S & F & V & K & V & R & I & N\end{array}$ GAA CAC AGA GAG AAC CTT GAC CCG TCA TCA CCG AGA GAC TAC ATT GAC TCC TTC CTT ATA 840 $\begin{array}{llllllllllllllllllll}\mathrm{E} & \mathrm{H} & \mathrm{R} & \mathrm{E} & \mathrm{N} & \mathrm{L} & \mathrm{D} & \mathrm{P} & \mathrm{S} & \mathrm{S} & \mathrm{P} & \mathrm{R} & \mathrm{D} & \mathrm{Y} & \mathrm{I} & \mathrm{D} & \mathrm{S} & \mathrm{F} & \mathrm{L} & \mathrm{I}\end{array}$ GAG ATG GGA GAG AAG GAA GAC AAA GAT TCT GGA TTT GAT CTC GAC AAT TTG TGC TTT TGC 900 $\begin{array}{llllllllllllllllllll}E & M & G & E & K & E & D & K & D & S & G & F & D & L & D & N & L & C & F & C\end{array}$ GTT CTG GAC CTT TTT GTT GCT GGA ACC GAA ACC ACC ACC ACT ACT TTA CAC TGG GGA CTG 960 $\begin{array}{llllllllllllllllllll}V & L & D & L & F & V & A & G & T & E & T & T & T & T & T & L & H & W & G & L\end{array}$ CTG TAC ATG ATC TGC AAC CCA CAG ATA CAA GAG CGT GTC CAG GCT GAA ATA GAT GCC GTG 1020 $\begin{array}{llllllllllllllllllll}I & Y & M & I & C & N & P & Q & I & Q & E & R & V & Q & A & E & I & D & A & V\end{array}$ ATT GGT CCA TCC AGG CCG CCC TCT ATG TCT GAC AGA GAC AAC ATG CCC TAC ACT GAT GCA 1080 $\begin{array}{llllllllllllllllllll}I & G & P & S & R & P & P & S & M & S & D & R & D & N & M & P & Y & T & D & A\end{array}$ GTC ATC CAT GAG ATA CAG AGG ATG GGT AAC ATC ATC CCC CTC AAC GTG GCT CGC ATG GCT 1140 $\begin{array}{llllllllllllllllllll}V & I & H & E & I & Q & R & M & G & N & I & I & P & L & N & V & A & R & M & A\end{array}$ AAC AAA GAC ACC ACA GTG GAT CAA TAC ACC ATC CCA AAG nnn nnn nnn nnn nnn GCT ACA 1200 $\begin{array}{llllllllllllllllllll}N & K & D & T & T & V & D & Q & Y & T & I & P & K & X & X & X & X & X & A & T\end{array}$ CTG GAC TCA GTT CTA CAT GAT GAG TCC ATG TGG GAA ACT CCA AAC ACC ATC AAT CCG GAG 1260 $\begin{array}{llllllllllllllllllll}L & D & S & V & I & H & D & E & S & M & W & E & T & P & N & T & I & N & P & E\end{array}$ CAC TTT TTG GAA AAG GAC GGC ACA TTC AGG AAG AGA GAG GCC TTT CTT CCC TTC TCT GCA 1320 $\begin{array}{llllllllllllllllllll}\mathrm{H} & \mathrm{F} & \mathrm{L} & \mathrm{E} & \mathrm{K} & \mathrm{D} & \mathrm{G} & \mathrm{T} & \mathrm{F} & \mathrm{R} & \mathrm{K} & \mathrm{R} & \mathrm{E} & \mathrm{A} & \mathrm{F} & \mathrm{L} & \mathrm{P} & \mathrm{F} & S & \mathrm{~A}\end{array}$

Figure 6. Nucletoide and predicted amino acid sequence for killifish CYP2P1 exons 1-8. The same features are highlighted as described in the legend to Figure 1. The ns and the Xs denote missing sequence rather than ambiguous sequence. Exon 9 has not yet been sequenced. 
OLERSIAR TAESIS

NO PAGE "86 
CHAPTER 3

FUNCTIONAL CHARACTERISTICS OF NOVEL CYTOCHROMES P450 (CYP2N1 and CYP2N2) HIGHLY EXPRESSED IN KILLIFISH HEART AND BRAIN 


\section{$\underline{\text { ABSTRACT }}$}

The CYP2N subfamily recently identified in the teleost Fundulus heteroclitus (killifish) contains at least two genes, CYP2N1 and CYP2N2. Particularly noteworthy is the cloning of CYP2N2 from heart and the high expression of its mRNA in heart and brain. CYP2N1 transcripts are expressed primarily in liver and gut but also are expressed in heart and brain (Oleksiak et al., in preparation). This tissue distribution suggests that these heme thiolate proteins might have an endogenous function. Arachidonic acid is potentially an endogenous substrate for the CYP2N P450s because the fish CYP2Ns are related most closely to the mammalian CYP2Js based on their phylogeny and tissue distribution (Oleksiak et al., in preparation), and several members of the CYP2J subfamily metabolize arachidonic acid (Wu et al., 1996; Wu et al., 1997; Zhang et al., 1997). To test this hypothesis, CYP2N1 and CYP2N2 were co-expressed with NADPH cytochrome P450 reductase in insect cells using a baculovirus system, and their ability to metabolize arachidonic acid was examined. CYP2N1 metabolized arachidonic acid more than four times faster than CYP2N2 (catalytic turnover: 435 and $95 \mathrm{pmol} / \mathrm{nmol} \mathrm{P} 450 / \mathrm{min}$ for CYP2N1 and CYP2N2, respectively). Both CYP2N1 and CYP2N2 catalyzed the NADPH-dependent, regio- and enantioselective epoxidation of arachidonic acid to $11(\mathrm{R})$, 12(S)-epoxyeicosatrienoic acid (EET) (optical purity: $92 \%$ and $70 \%$ for CYP2N1 and CYP2N2, respectively) and to $8(\mathrm{R}), 9(\mathrm{~S})$-epoxyeicosatrienoic acid (optical purity: $91 \%$ and $90 \%$ for CYP2N1 and CYP2N2, respectively). CYP2N1 also produced racemic 14,15-EET while CYP2N2 produced 14(S),15(R)-EET (optical purity: 68\%). In addition, CYP2N1 hydroxylated arachidonic acid to a variety of mid-chain, cis-trans-conjugated dienols (5-, 16-, 12-, 9-, and 11-hydroxyeicosaetraenoic acid (HETE) in order of decreasing amounts) while CYP2N2 mainly produced 9-, an unidentified-, and 20hydroxyeicosatetraenoic acid (also in order of decreasing amounts). Killifish liver 
microsomes metabolized arachidonic acid primarily to epoxyeicosatrienoic acids and their hydration products, dihydroxyeicosatrienoic acids (DHETs). Most of these products could result from the metabolic activities of CYP2N1 and CYP2N2. The results demonstrate a catalytic diversity of arachidonic acid metabolites in early vertebrates and suggest that the novel CYP2N genes may encode early vertebrate forms of arachidonic acid epoxygenases and hydroxylases. Functional studies with CYP2N1 and CYP2N2 may elucidate the fundamental roles of their activities in vertebrates, particularly in organs such as heart and brain.

\section{INTRODUCTION}

Since it was first suggested in 1961 that the enzyme involved in the $\omega$-oxidation of fatty acids might operate as a mixed function oxidase (Wakabayashi and Shimazono, 1961), numerous studies have focused on the cytochrome P450 mediated metabolism of fatty acids. Initial studies were done to examine the metabolism of saturated fatty acids (Verkade and Lee, 1934; Lu and Coon, 1968; Lu et al., 1969). Given the broad substrate specificities of many of the cytochrome $\mathrm{P} 450$ proteins, not surprisingly, cytochromes $\mathrm{P} 450$ soon were shown to metabolize unsaturated fatty acids as well (Oliw, 1994). Arachidonic acid metabolism has been studied most intensely because of its importance as a precursor to signaling molecules in mammals.

The first report suggesting a biological function for cytochrome P450 metabolites of arachidonic acid showed that cytochrome $\mathrm{P} 450$ inhibitors blocked arachidonic acid induced platelet aggregation and the formation of aggregation factors from arachidonic acid by platelet microsomal P450s (Cinti and Feinstein, 1976). Subsequent studies have described the various arachidonic acid metabolites formed by cytochromes P450 (Capdevila et al., 1981; Morrison and Pascoe, 1981; Oliw et al., 1982), their biological activities, and the 
potential physiological importance of these metabolites (Fitzpatrick and Murphy, 1989; Proctor et al., 1989; McGiff, 1991). The epoxygenase reaction has been studied most intensely because the EETs have been shown to be endogenous constituents of numerous tissues and have been shown to have potent biological activities (Capdevila et al., 1984; Fitzpatrick and Murphy, 1989). Lipooxygenase hydroxy-metabolites of arachidonic acid also have been shown to be endogenous constituents of numerous tissues (Spector et al., 1988), and recent identification of specific CYP that have hydroxylase activities towards arachidonic acid might highlight important biological activities for these metabolites as well. These cytochrome P450/arachidonic acid studies have concentrated on mammals with few exceptions (Gilday et al., 1996). No studies have fully addressed the importance of cytochrome $\mathrm{P} 450$ arachidonic acid metabolites in other vertebrates.

Fish are the earliest diverging vertebrates. Comparisons between these earlier diverging vertebrates and later diverging mammalian species address important questions of functional conservation and divergence. Important endogenous functions in fish likely are maintained in mammals. Hence, fish are a logical starting point to address questions of functional significance. Some of the most interesting questions concerning cytochrome P450 proteins consider their endogenous functions, and looking at CYP in early diverging vertebrates may be a useful approach towards unraveling endogenous CYP functions. The compelling question concerning the teleost CYP2N proteins addresses their endogenous functions.

CYP2N1 recently was cloned from a killifish liver cDNA library, and CYP2N2 recently was cloned from a killifish heart cDNA library (Oleksiak et al., in preparation). The high expression of CYP2N2 transcripts in heart and brain as well as the expression of CYP2N1 transcripts in heart and brain suggests that the CYP2Ns might have endogenous functions in these tissues. The CYP2N transcripts also are expressed in liver and gut. Because the evolution of the nervous system was dependent on the co-evolution of the 
vasculature for a supply of nutrients and oxygen (Crawford and Harbige, 1988), the presence of the CYP2Ns in brain, gut and heart is highly intriguing.

The phylogenetic relationships of the CYP2Ns and their tissue distributions have suggested possible homologies in the mammalian CYP2s. Based on sequence similarities, the mammalian CYP to which the CYP2Ns are related most closely are the CYP2Js. The CYP2Ns also share similar tissue distributions to the CYP2Js. CYP2N1 transcripts are expressed most highly in liver and gut and also are expressed in heart and brain. CYP2N2 transcripts are expressed most highly in heart and brain and also are expressed in liver, gut, eye, gill, and kidney (Oleksiak et al., in preparation). Similarly, the CYP2J transcripts all are expressed in liver and gut and some are expressed in heart (Kikuta et al., 1991; Wu et al., 1996; Wu et al., 1997; Zhang et al., 1997). Because several members of the CYP2J subfamily metabolize arachidonic acid (Wu et al., 1996; Wu et al., 1997; Zhang et al., 1997), arachidonic acid is potentially an endogenous substrate for the CYP2N P450s as

well. Analysis of the ability of the CYP2Ns to metabolize arachidonic acid is a starting point to address potential conserved endogenous functions for these heme thiolate proteins in heart and brain.

\section{MATERIALS AND METHODS}

Materials: $[1-14 \mathrm{C}$ ]arachidonic acid was purchased from DuPont NEN. Triphenylphosphine, $\alpha$-bromo-2,3,4,5,6-pentafluorotoluene, N,N-diisopropylethylamine, and diazald were purchased from Aldrich. All other chemicals and reagents were purchased from Sigma unless otherwise specified.

Heterologous Expression of Recombinant CYP2N1 and CYP2N2: The proteins encoded by the CYP2N1 or CYP2N2 cDNAs were co-expressed with human NADPH cytochrome P450 reductase (CYPOR) in Sf9 insect cells using the pAcUW51-CYPOR 
shuttle vector (kindly provided by Dr. Cosette Serabjit-Singh, Glaxo Research Institute, Research Triangle Park, NC) (Lee et al., 1995) and the BaculoGold Baculovirus Expression System (Pharmingen, San Diego, CA). Briefly, the CYP2N1 or CYP2N2 cDNA was cut from the General Contractor pCNTR cloning vector with BamHI and ligated into the pAcUW51-CYPOR linearized with BamHI. In the resulting expression vectors (pAcUW51-CYPOR-CYP2N1 and pAcUW51-CYPOR-CYP2N2), the p10 promoter controlled the expression of CYPOR and the polyhedrin vector independently controlled the expression of the CYP2N1 or CYP2N2. Cultured Sf9 insect cells were co-transfected with each of the transfer vectors independently (pAcUW51-CYPOR-CYP2N1 and pAcUW51CYPOR-CYP2N2) and linearized wild-type BaculoGold viral DNA in a $\mathrm{CaCl}_{2}$ solution. Recombinant viruses were purified and the presence of the CYP2N1 and CYP2N2 cDNAs corroborated by PCR analysis. Cultured $S f 9$ cells, grown in spinner flasks at a density of 1.5-2.0 X $10^{6}$ cells/ml, were infected with a high titer CYP2N1-CYPOR or CYP2N2CYPOR recombinant stock with $5 \mathrm{uM}$ hemin or $5 \mathrm{uM} \delta$-aminolevulenic acid hydrochloride (Gonzalez, 1991). Cells co-expressing recombinant CYP2N1 and CYPOR or recombinant CYP2N2 and CYPOR were harvested 72 hours after infection, washed twice with phosphate buffered saline, and used to prepare microsomal fractions by differential centrifugation at $4^{\circ} \mathrm{C}$ as described previously (Zeldin et al., 1995). The P450 content was determined spectrally according to the method of Omura and Sato (Omura and Sato, 1964) using a Shimadzu UV-3000 dual wavelength/double-beam spectrophotometer (Shimadzu Scientific Instruments, Columbia, MD).

Reconstitution of Recombinant Activity and Product Characterization: Microsomes were resuspended to a final reaction volume in $0.05 \mathrm{M}$ Tris- $\mathrm{Cl}$ buffer ( $\mathrm{pH} 7.5$ ) containing $0.15 \mathrm{M} \mathrm{KCl}, 0.01 \mathrm{M} \mathrm{MgCl}_{2}, 8 \mathrm{mM}$ sodium isocitrate, and $0.5 \mathrm{IU}$ of isocitrate dehydrogenase $/ \mathrm{ml}$ (as an NADPH regenerating system) and equilibrated at $37^{\circ} \mathrm{C}$ with constant mixing for two minutes before the addition of $[1-14 \mathrm{C}]$ arachidonic acid $(25-55$ 
$\mathrm{uCi} / \mathrm{umol}, 70-100 \mathrm{uM}$ final concentration). Reactions were initiated by the addition of NADPH ( $1 \mathrm{mM}$ final concentration) and continued at $37{ }^{\circ} \mathrm{C}$ for one hour with constant mixing. Reaction products were extracted into ethyl ether, dried under nitrogen, resolved by reverse-phase HPLC, and quantified by on-line liquid scintillation using a Radiomatic Flo-One B-detector (Radiomatic Instruments, Tampa, FL). Products were identified by comparing their reverse-phase and normal phase HPLC properties with those of authentic standards and by GC/MS (Capdevila et al., 1986; Capdevila et al., 1990; Clare et al., 1991). For chiral analysis, the EETs were collected from the HPLC eluent, derivatized to the corresponding EET-PFB or EET-methyl esters, purified by normal-phase HPLC, resolved into the corresponding antipodes by chiral-phase HPLC, and quantified by liquid scintillation as described previously (Capdevila et al., 1991; Hammonds et al., 1989). For HETE identifications, HETEs were collected from the HPLC eluent and were identified by normal phase HPLC as described (Capdevila et al., 1982; Capdevila et al., 1985; Falck et al., 1990). Uninfected $S f 9$ cells and reactions without the addition of NADPH were used as negative controls.

Benzphetamine $\mathrm{N}$-demethylase activities: The benzphetamine $\mathrm{N}$-demethylase activities of the recombinant CYP were assessed using the same microsomal preparations used for arachidonic acid assays under identical reaction conditions but employing benzphetamine ( $2 \mathrm{mM}$ final concentration) as the substrate. The reaction product (formaldehyde) was quantified according to the method of Nash (Nash, 1953).

O-deethylase activities: The ethoxyresorufin, benzyloxyresorufin, methoxyresorufin, and pentoxyresorufin O-deethylase activities of the recombinant CYP were assessed using the same microsomal preparations used for arachidonic acid and benzphetamine assays. The reaction product, resorufin, was quantified using a cytofluor as described (Hahn et al., 1993). 
Synthetic Procedures: The $[1-14 \mathrm{C}] \mathrm{EET}$ internal standards were synthesized from [1-14 $\mathrm{C}]$ arachidonic acid (55-57 uCi/umol) by nonselective epoxidation as described (Falck et al., 1990). Racemic and enantiometricaly pure EETs were prepared by total chemical synthesis according to published procedures (Corey et al., 1980; Falck and Manna, 1982; Mosset et al., 1986; Moustakis et al., 1986). Methylations were performed using an ethereal solution of diazomethane (Capdevila et al., 1986). PFB esters were formed by reaction with pentafluorobenzyl bromide as described (Karara et al., 1989). TMS ethers were prepared using 25\% (v/v) bis(trimethylsilyl)trifluoracetamide in anhydrous pyridine (Porter et al., 1979). HETE standards were purchased from Cayman Chemical Company. HETEs and C-19/C-20 alcohols of arachidonic acid were synthesized as described (Manna et al., 1983).

Statistical Methods: All values are expressed as mean $+/-$ standard error of the mean (SEM).

\section{RESULTS}

Expression of Recombinant CYP2N1 and CYP2N2: The teleost CYP2N proteins were co-expressed with CYPOR in $S f 9$ insect cells using the baculovirus expression system according to previously described methods (Gonzalez et al., 1991; Lee et al., 1995; Wu et al., 1996). Under the conditions outlined in the Materials and Methods, the level of expression of recombinant CYP2N was 3-10 nmol P450/liter of infected Sf9 cells. These recombinant CYP2N1 and CYP2N2 showed a typical CYP Soret absorption at $\sim 448 \mathrm{~nm}$ when reduced and complexed with carbon monoxide (Figures 1a and 1b). 


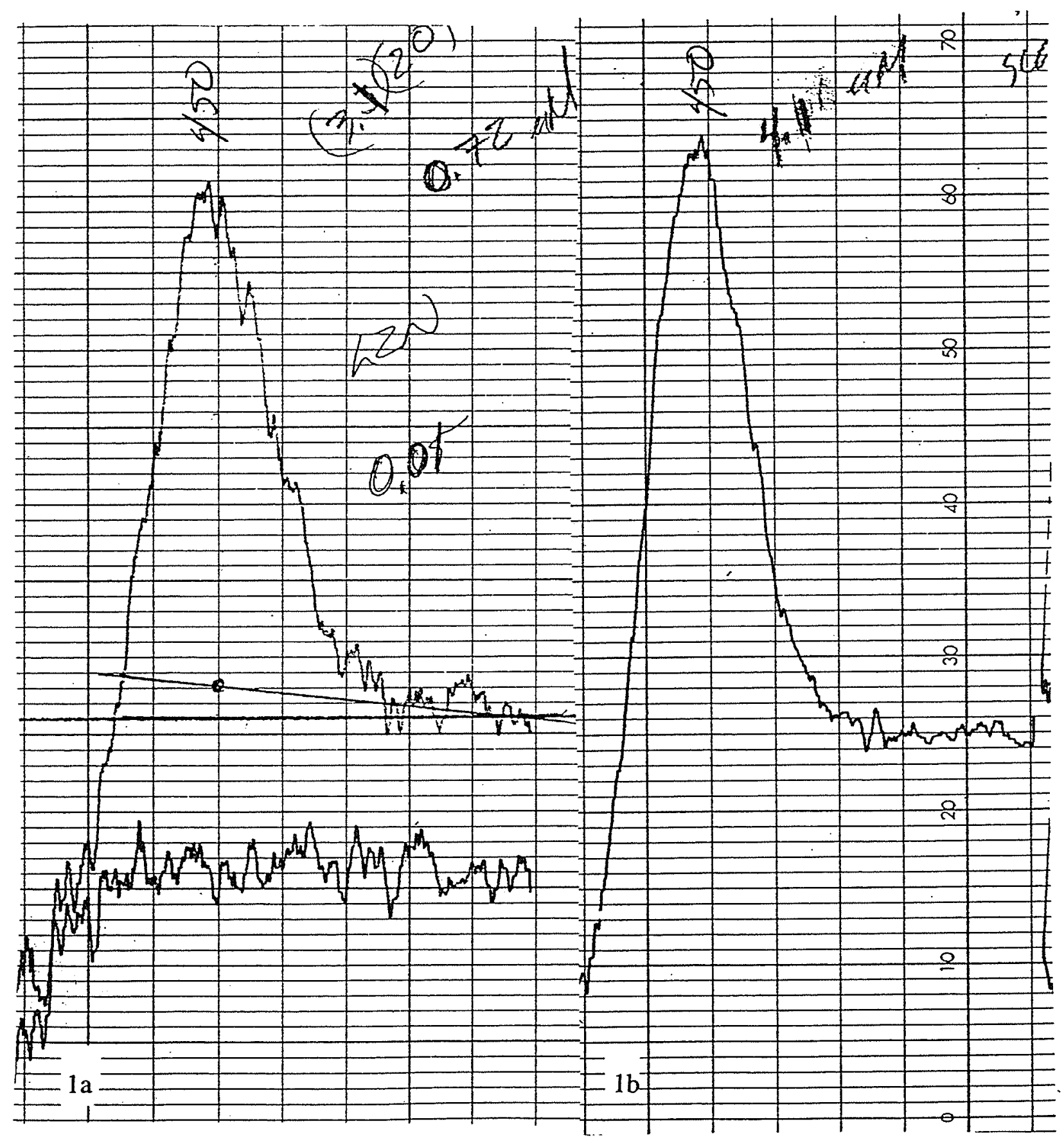

Figures 1a and 1b. Spectrophotometric analysis of CYP2N1 (1a) and CYP2N2 (1b) expressed in $S f 9$ microsomes. P450 content was analyzed by measuring the dithionite difference spectrum in CO-treated microsomes. 
Metabolism of Arachidonic Acid by CYP2N1: Microsomal fractions containing both the CYPOR and CYP2N1 recombinant proteins were incubated with arachidonic acid in the presence of NADPH and an NADPH regenerating system. As shown in Figure 2, CYP2N1 metabolized arachidonic acid to 14,15-, 11,12- and 8,9-EETs and to 16-, 5-, 12-, 9-, and 11-HETEs, in order of decreasing amounts, as the major reaction products with a rate of $435 \mathrm{pmol}$ of product formed $/ \mathrm{nmol} \mathrm{P} 450 / \mathrm{min}$ at $37^{\circ} \mathrm{C}$. We identified each of the major products by comparing their HPLC properties with those of authentic standards and by gas chromatography/mass spectroscopy. None of these metabolites were formed in the absence of NADPH showing that the reaction was P450 mediated (Figure 2).

As illustrated in Figure 2, CYP2N1 is both an arachidonic acid epoxygenase and hydroxylase. Regiochemical analysis of the EETs revealed a strong preference for epoxidation at the 8,9-olefin (59\% of total EET products) (Table 1). Epoxidation at the 11,12- and 14,15-olefins occurred less often (29\% and $12 \%$ of total EET products, respectively). Stereochemical analysis of the CYP2N1-derived EETs revealed a high preference for $11(\mathrm{R}), 12(\mathrm{~S})$ - and $8(\mathrm{R}), 9(\mathrm{~S})$-EETs (optical purities $92 \%$ and $91 \%$, respectively) (Table 1). The stereochemistry of the 14,15-EET was practically racemic (Table 1). Regiochemical analysis of the HETEs revealed a preference for hydroxylation to form 5-HETE followed by 16-, 12-, 9-, and 11-HETEs (37\%, 23\%, 16\%, 6\%, and 4\%, respectively) (Table 2).

Metabolism of Arachidonic Acid by CYP2N2: Microsomal fractions containing both the CYPOR and CYP2N2 recombinant proteins were incubated with arachidonic acid using conditions identical to those for CYP2N1. The chromatogram in Figure 3 shows that, similar to CYP2N1, CYP2N2 is both an arachidonic epoxygenase and hydroxylase. CYP2N2 metabolized arachidonic acid to 14,15-, 11,12- and 8,9-EETs and to 20-HETE, 9-HETE and an unidentified HETE as the major reaction products (catalytic turnover: 95 pmol of product formed/nmol P450/min at $37^{\circ} \mathrm{C}$ ). As with CYP2N1, each of the major 

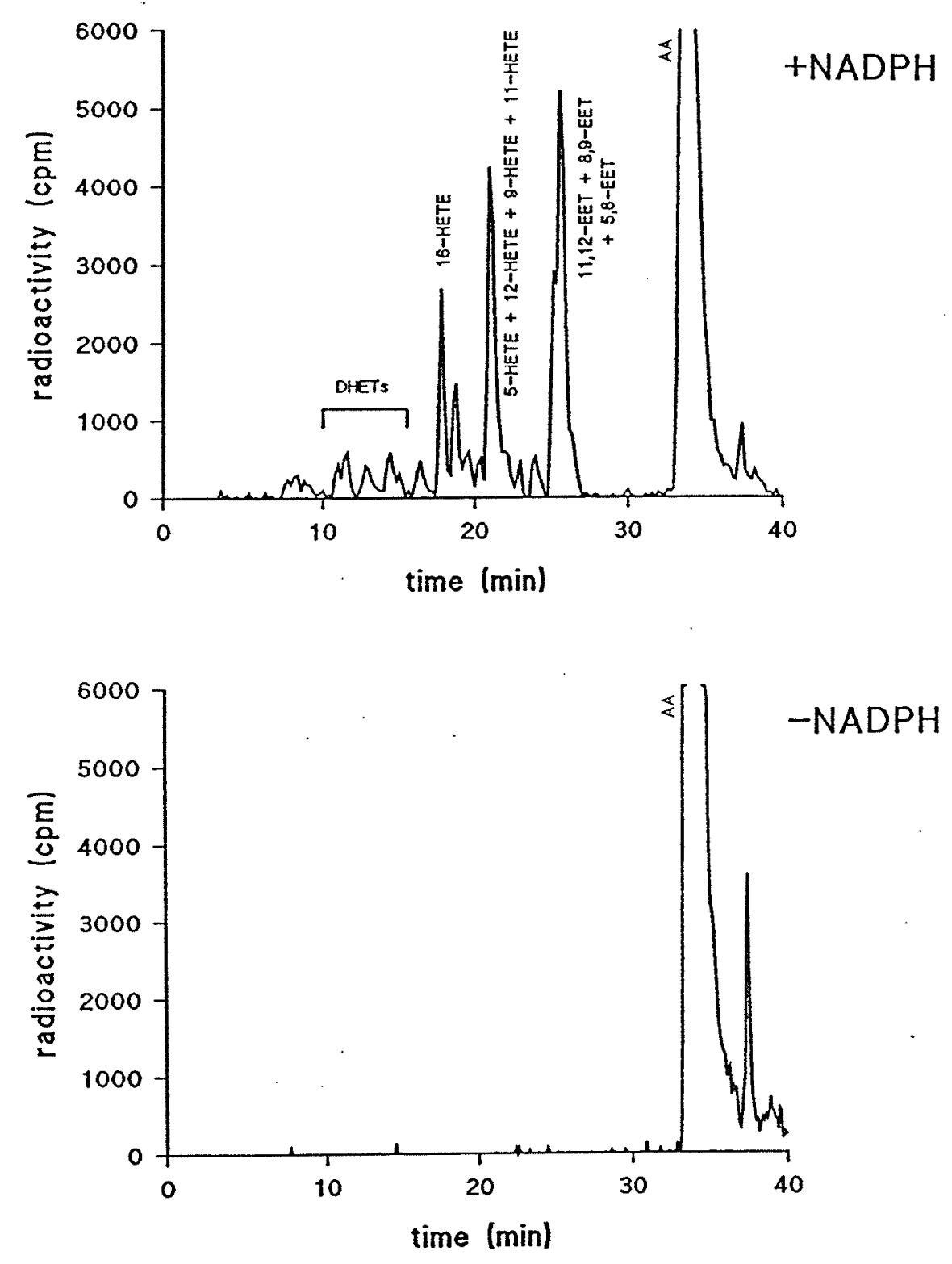

Figure 2. Reverse-phase HPLC chromatogram of the organic soluble metabolites generated during incubation of recombinant CYP2N1 with $[1-14 \mathrm{C}]$ arachidonic acid. Microsomal fractions prepared from CYP2N1/CYPOR transfected $S f 9$ insect cells were incubated at $37^{\circ} \mathrm{C}$ with $[1-14 \mathrm{C}]$ arachidonic acid (70-100 uM, final concentration) with/without NADPH (1 mM, final concentration) and an NADPH regenerating system. After 1 hour, the reaction products were extracted and resolved by reversed-phase HPLC as described. Peak identifications were made by comparisons of the HPLC properties of individual peaks with those of authentic standards using both reverse-phase and normalphase HPLC and by GC/MS. Ordinate: radioactivity in cpm; abscissa: time in min. Top panel: incubation with NADPH; bottom panel: incubation without NADPH. 
Table 1.

Regio- and stereochemical composition of EETs produced by recombinant CYP2N1.

\begin{tabular}{lccc}
\hline Regioisomer & Distribution (\% total EETs) & & \multicolumn{2}{c}{ Enantioselectivity } \\
& & & R, R \\
\hline 14,15-EET & 12 & 49 & 51 \\
$11,12-\mathrm{EET}$ & 29 & 92 & 8 \\
$8,9-\mathrm{EET}$ & 59 & 91 & 9 \\
$5,6-\mathrm{EET}$ & $\mathrm{ND}$ & & \\
\end{tabular}

ND: not detected

The activity of recombinant CYP2N1 was reconstituted in the presence of NADPH and an NADPH regenerating system as described in Materials and Methods. After one hour, the EET products were extracted into ethyl ether, resolved into individual regioisomers by reverse and normal-phase HPLC, derivatized to corresponding EET-PFB or EET-methyl esters, purified by normal-phase HPLC, and resolved into the corresponding antipodes by chiral -phase HPLC. Values shown are averages of at least three different experiments with $\mathrm{SEM}<5 \%$. 
Table 2.

Regiochemical composition of HETEs produced by recombinant CYP2N1.

\begin{tabular}{rc}
\hline Regioisomer & Distribution (\% total HETEs) \\
& \\
5-HETE & 37 \\
16-HETE & 23 \\
12-HETE & 16 \\
9-HETE & 6 \\
11-HETE & 4 \\
\hline
\end{tabular}

The activity of recombinant CYP2N1 was reconstituted in the presence of NADPH and an NADPH regenerating system as described in Materials and Methods. After one hour, the HETE products were extracted into ethyl ether and resolved into individual regioisomers by reverse and normal-phase HPLC. Values shown are averages of at least three different experiments with $\mathrm{SEM}<5 \%$. 
products was identified by comparing its HPLC properties with those of authentic standards and by gas chromatography/mass spectroscopy. None of these metabolites were formed in the absence of NADPH showing that the reaction was P450 mediated (Figure 3).

CYP2N2 epoxidated arachidonic acid preferentially at the 8,9-olefin which made up $67 \%$ of the total EET products and less frequently at the 11,12- and 14,15-olefins (19\% and $15 \%$ of total EET products, respectively) (Table 3). Stereochemical analysis of the CYP2N2-derived EETS showed that the 8,9-EET was formed with a very high specificity for the $8(R), 9(S)$ isomer showing an optical purity of $90 \%$, similar to the stereospecificity of CYP2N1. CYP2N2 also preferentially made 11(R),12(S)-EET with an optical purity of $70 \%$, a lower specificity than CYP2N1. The 14,15-EET was formed with a preference for $14(\mathrm{~S}), 15(\mathrm{R})$-EET with an optical purity of $68 \%$. CYP2N2 predominantly formed 9HETE, 30\% of the total HETE products, and also formed an unidentified HETE and 20HETE, $25 \%$ and $12 \%$ of the total HETE products, respectively (Table 4).

Activity of liver microsomes: Liver microsomal fractions were incubated with arachidonic acid in the presence of NADPH and an NADPH regenerating system. The chromatogram in Figure 4 shows that killifish microsomes exhibit both epoxygenase and hydroxylase activities towards arachidonic acid. Killifish liver microsomes metabolized arachidonic acid primarily to 14,15-, 11,12-, and 8,9-DHETs which are hydration products of the 14,15-, 11,12-, and 8,9-EETs, and to 19- and 16-HETEs. The killifish microsomes metabolized arachidonic acid with a rate of $210 \mathrm{pmol}$ of product formed/nmol P450/min at $37^{\circ} \mathrm{C}$. Regiochemical analysis of the DHETs revealed a preference for epoxidation and subsequent hydration at the 8,9 -olefin which made up $61 \%$ of total DHET products (Table 5). Epoxidation and subsequent hydration at the 11,12- and 14,15-olefins occurred less often (24\% and $15 \%$ of total DHET products, respectively). Regiochemical analysis of the HETEs revealed a preference for hydration to form 16- and 19-HETEs (46\% and $8 \%$, respectively) (Table 6). 

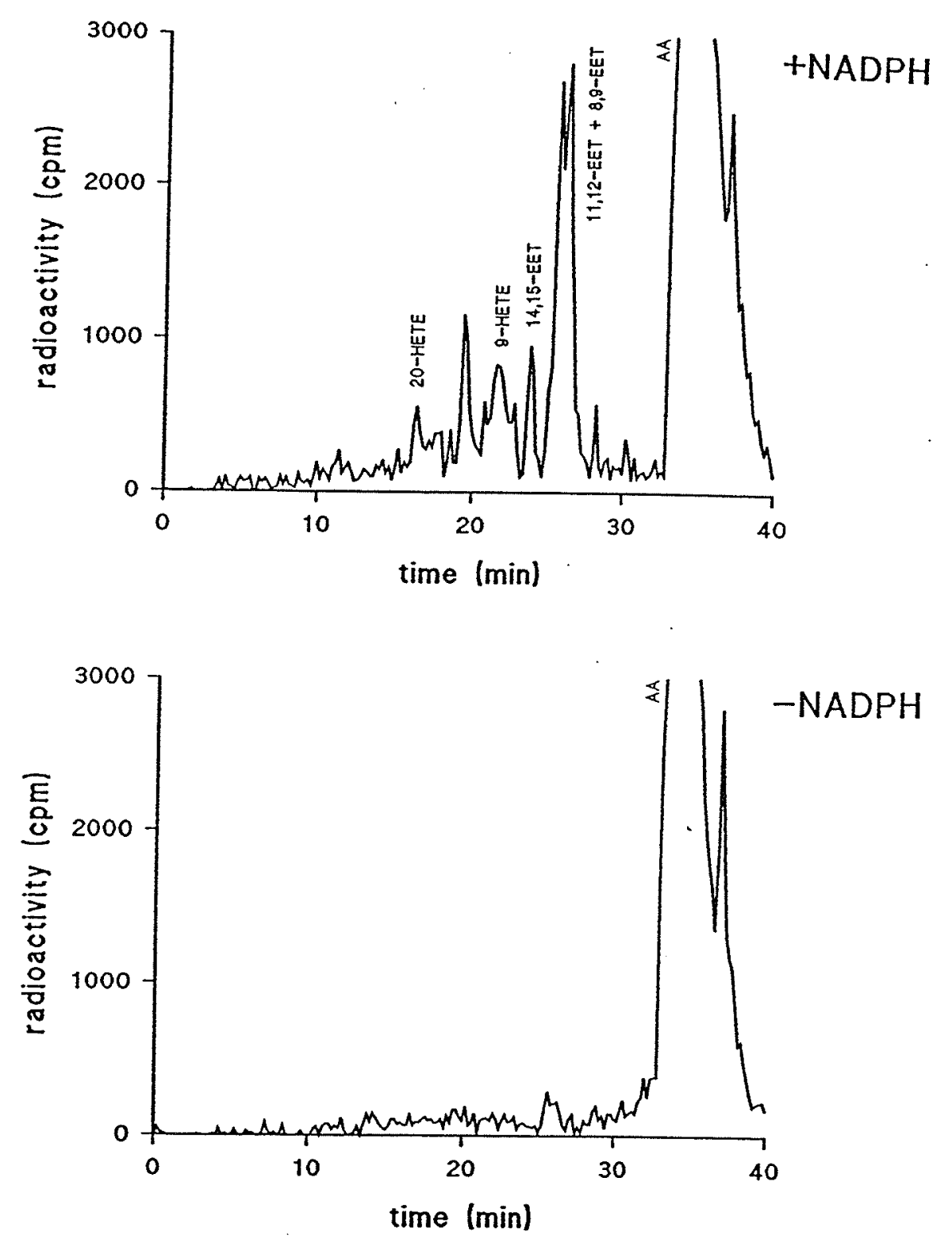

Figure 3. Reverse-phase HPLC chromatogram of the organic soluble metabolites generated during incubation of recombinant CYP2N2 with $\left[1-{ }^{14} \mathrm{C}\right]$ arachidonic acid. Microsomal fractions prepared from CYP2N2/CYPOR transfected $S f 9$ insect cells were incubated at $37^{\circ} \mathrm{C}$ with $[1-14 \mathrm{C}]$ arachidonic acid (70-100 uM, final concentration) with/without NADPH ( $1 \mathrm{mM}$, final concentration) and an NADPH regenerating system. After 1 hour, the reaction products were extracted and resolved by reversed-phase HPLC as described. Peak identifications were made by comparisons of the HPLC properties of individual peaks with those of authentic standards using both reverse-phase and normalphase HPLC and by GC/MS. Ordinate: radioactivity in cpm; abscissa: time in min. Top panel: incubation with NADPH; bottom panel: incubation without NADPH. 
Table 3.

Regio- and stereochemical composition of EETs produced by recombinant CYP2N2.

\begin{tabular}{lclc}
\hline Regioisomer & Distribution (\% total EETs) & \multicolumn{2}{c}{ Enantioselectivity } \\
& & R,S & S,R \\
\hline 14,15-EET & 15 & 32 & 68 \\
$11,12-\mathrm{EET}$ & 19 & 70 & 30 \\
$8,9-\mathrm{EET}$ & 67 & 90 & 10 \\
$5,6-\mathrm{EET}$ & $\mathrm{ND}$ & & \\
& & & \\
\hline
\end{tabular}

ND: not detected

The activity of recombinant CYP2N2 was reconstituted in the presence of NADPH and an NADPH regenerating system as described in Materials and Methods. After one hour, the EET products were extracted into ethyl ether, resolved into individual regioisomers by reverse and normal-phase HPLC, derivatized to corresponding EET-PFB or EET-methyl esters, purified by normal-phase HPLC, and resolved into the corresponding antipodes by chiral -phase HPLC. Values shown are averages of at least three different experiments with $\mathrm{SEM}<5 \%$. 
Table 4.

Regiochemical composition of HETEs produced by recombinant CYP2N2.

Regioisomer Distribution (\% total HETES)

9-HETE 30

unidentified-HETE 25

20-HETE 12

The activity of recombinant CYP2N2 was reconstituted in the presence of NADPH and an NADPH regenerating system as described in Materials and Methods. After one hour, the HETE products were extracted into ethyl ether and resolved into individual regioisomers by reverse and normal-phase HPLC. Values shown are averages of at least three different experiments with $\mathrm{SEM}<5 \%$. 

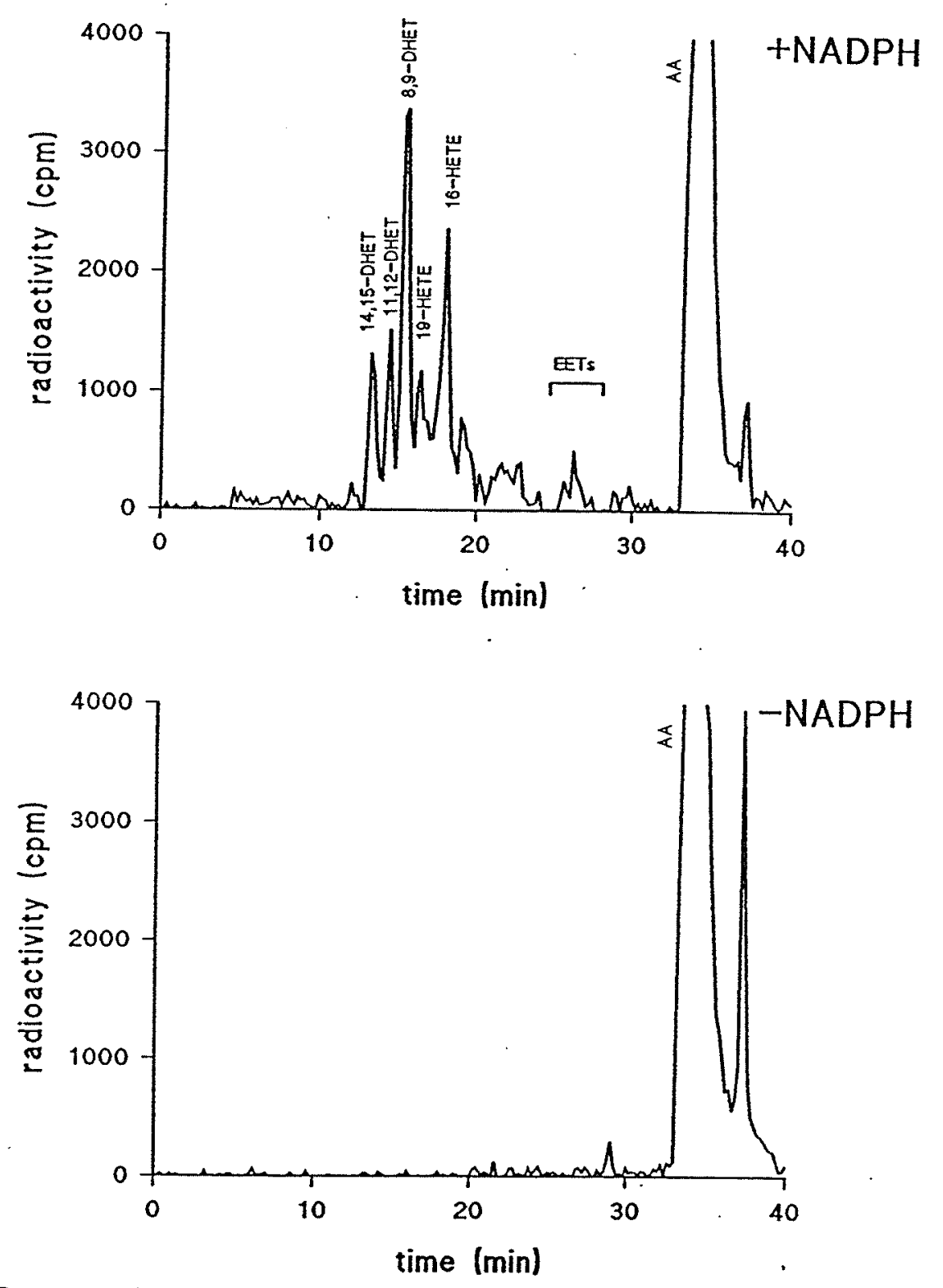

Fig. 4. Reverse-phase HPLC chromatogram of the organic soluble metabolites generated during incubation of killifish liver microsomes with $\left[1-{ }^{14} \mathrm{C}\right]$ arachidonic acid. Liver microsomal fractions were incubated at $37 \mathrm{C}$ with $[1-14 \mathrm{C}]$ arachidonic acid $(70-100 \mathrm{uM}$, final concentration) with/without NADPH ( $1 \mathrm{mM}$, final concentration) and an NADPH regenerating system. After 1 hour, the reaction products were extracted and resolved by reversed-phase HPLC as described. Peak identifications were made by comparisons of the HPLC properties of individual peaks with those of authentic standards using both reversephase and normal-phase HPLC and by GC/MS. Ordinate: radioactivity in cpm; abscissa: time in min. Top panel: incubation with NADPH; bottom panel: incubation without NADPH. 
Table 5.

Regiochemical composition of DHETs produced by killifish microsomes.

\begin{tabular}{lc}
\hline Regioisomer & Distribution (\% total DHETs) \\
& \\
$14,15-\mathrm{DHET}$ & 15 \\
11,12 -DHET & 24 \\
8,9-DHET & 61 \\
5,6-DHET & $\mathrm{ND}$ \\
\hline
\end{tabular}

ND: not detected

The activity of killifish liver microsomes was reconstituted in the presence of NADPH and an NADPH regenerating system as described in Materials and Methods. After one hour, the DHET products were extracted into ethyl ether and resolved into individual regioisomers by reverse and normal-phase HPLC. Values shown are averages of at least three different experiments with $\mathrm{SEM}<5 \%$. 
Table 6.

Regiochemical composition of HETEs produced by killifish microsomes.

Regioisomer

16-HETE

19-HETE
Distribution (\% total HETEs)

46

8

The activity of killifish liver microsomes was reconstituted in the presence of NADPH and an NADPH regenerating system as described in Materials and Methods. After one hour, the HETE products were extracted into ethyl ether and resolved into individual regioisomers by reverse and normal-phase HPLC. Values shown are averages of at least three different experiments with $\mathrm{SEM}<5 \%$. 
Benzphetamine $\mathrm{N}$-demethylase Activities: The catalytic properties of recombinant CYP2N1 and CYP2N2 and killifish liver microsomes towards benzphetamine were examined using portions of the same microsomal preparations that were used for arachidonic acid studies. The baculovirus-expressed CYP2N1 had substantial demethylase activity of $1.0 \mathrm{nmol} / \mathrm{nmol} \mathrm{P} 450 / \mathrm{min}$., and CYP2N2 demonstrated a similar demethylase activity of $0.8 \mathrm{nmol} / \mathrm{nmol} \mathrm{P} 450 / \mathrm{min}$. (Table 7). Killifish liver microsomes also demethylated benzphetamine at $0.5 \mathrm{nmol} / \mathrm{nmol} \mathrm{P} 450 / \mathrm{min}$. (Table 7).

O-deethylase Activities: Similarly, the alkoxyresorufin O-deethylase activities of CYP2N1, CYP2N2, and killifish liver microsomes were compared with microsomes incubated with NADPH and ethoxyresorufin, pentoxyresorufin, benzyloxyresorufin, or methoxyresorufin. Relative to activities of killifish liver microsomes, these recombinant CYP showed minimal ethoxy- and methoxy-O-deethylase activities (Table 7). However, the rates of benzyloxy-and pentoxy-O-deethylase activities catalyzed by the expressed CYP2Ns indicate that these proteins may contribute significantly to these activities in liver.

\section{DISCUSSION}

Recent studies have identified a number of cytochromes P450 in fish, defining new CYP2 subfamilies, CYP2N and CYP2P (Oleksiak et al., in preparation). A fundamental question is whether or not these CYP2 proteins are unique to fish. If so, why? If not, which CYP are they related to functionally? The tissue distribution of these CYP begins to address their functions; high expression of CYP2N transcripts in the heart and brain of untreated killifish suggests that the CYP2N proteins have more specialized functions than those CYP that are predominantly hepatic and which are mainly involved in detoxication. Expression of CYP2N transcripts in these tissues raises the question of the endogenous functions of the CYP2N proteins. We have begun to address this by examining the 
Table 7.

Metabolic rates (pmol/min/nmol P450) of recombinant CYP2N1, CYP2N2, and killifish liver microsomes incubated with different substrates.

\begin{tabular}{lrrrrrr}
\hline & AA & Benz. & ER & MR & BR & PR \\
\hline & & & & & & \\
CYP2N1 & 435 & 1000 & 3 & 2 & 8 & 2 \\
CYP2N2 & 95 & 840 & 1 & 1 & 4 & ND \\
liver microsomes & 210 & 470 & 1463 & 538 & 24 & 9 \\
& & & & & & \\
\hline
\end{tabular}

AA: arachidonic acid; Benz.: benzphetamine; ER: ethoxyresorufin; MR: methoxyresorufin; BR: benzyloxyresorufin; PR: pentoxyresorufin; ND: not detected.

The activities of recombinant CYP2N1, CYP2N2, and killifish liver microsomes were reconstituted in the presence of NADPH and an NADPH regenerating system as described in Materials and Methods. Values shown are averages of at least three different experiments with $\mathrm{SEM}<15 \%$. 
catalytic activities of these expressed proteins.

Our phylogenetic analyses of the CYP2N genes showed that they clustered with the mammalian CYP2Js. The CYP2N genes are related to genes in the CYP2J subfamily based not only on their sequences, but also on their tissue distributions. Many members of the CYP2J subfamily metabolize arachidonic acid. Accordingly, we hypothesized that arachidonic acid would be a potential endogenous substrate of the CYP2Ns. CYP2N1 and CYP2N2 not only share $75 \%$ identity at the amino acid level, they also display similar arachidonic acid metabolic profiles. Both of these CYP form predominantly epoxide products from arachidonic acid, and they form similar amounts of the different EET regioisomers (Table $8 \mathrm{a})$.

The enantioselectivities of the 8,9-EET products formed by CYP2N1 and CYP2N2 are almost identical with $91 \%$ and $90 \% 8(\mathrm{R}), 9(\mathrm{~S})$-EET formed by CYP2N1 and CYP2N2 respectively. The stereoselectivities of their 11,12-EET products are similar, but CYP2N1 forms 11(R),12(S)-EET with a much higher stereoselectivity of 92\%; CYP2N2 forms 11(R),12(S)-EET with only $70 \%$ stereoselectivity. In contrast, the 14,15-EET formed by CYP2N1 is practically racemic while CYP2N2 forms $14(\mathrm{~S}), 15(\mathrm{R})$-EET with $68 \%$ stereoselectivity (Table 8a).

The regiochemistry of the arachidonic acid metabolite profiles for killifish CYP2N1 and CYP2N2 reflects considerable similarities with the regiochemistry of the arachidonic acid metabolites formed by killifish liver microsomes. Liver microsomes also form predominantly epoxide products, DHETs, which are hydration products formed from the corresponding EETs by the subsequent action of epoxide hydrolases present in fish livers (Andersson et al., 1985). Although the stereochemical selectivities of the original EET regioisomers are unknown, the liver arachidonic acid DHET metabolites show a high similarity in distribution to the CYP2N1 and CYP2N2 epoxide metabolites; all make predominantly 8,9 -EET products followed by 11,12 - and then 14,15-EET products (Table 
Table 8a.

Comparison of the distribution and stereospecificities of the EETs or DHETs formed by recombinant CYP2N1, CYP2N2, and killifish liver microsomes.

\begin{tabular}{lcccccc}
\hline \multicolumn{5}{c}{ Distribution (\% total EETs or DHETs) and Stereospecificities } \\
CYP & 14,15 & $(\% \mathrm{R}, \mathrm{S})$ & 11,12 & $(\% \mathrm{R}, \mathrm{S})$ & 8,9 & $(\% \mathrm{R}, \mathrm{S})$ \\
\hline $\mathrm{N} 1$ & 12 & $(49)$ & 29 & $(92)$ & 59 & $(91)$ \\
$2 \mathrm{~N} 2$ & 15 & $(32)$ & 19 & $(70)$ & 67 & $(90)$ \\
$\begin{array}{l}\text { liver } \\
\text { microsomes }\end{array}$ & 15 & & 24 & & 61 & \\
\hline
\end{tabular}

Table $8 b$.

Comparison of the distribution of the HETEs formed by recombinant CYP2N1, CYP2N2, and killifish liver microsomes.

Distribution (\% total HETEs)

\begin{tabular}{lcccccccc} 
CYP & 5 & 9 & 11 & 12 & 16 & 19 & 20 & unidentified \\
\hline $2 \mathrm{~N} 1$ & 37 & 6 & 4 & 16 & 23 & & & \\
$2 \mathrm{~N} 2$ & & 30 & & & & & 12 & 25 \\
liver microsomes & & & & & 46 & 8 & &
\end{tabular}


8a). These similarities in regiochemical distribution as well as the high expression of CYP2N1 transcript in killifish liver suggest that the liver arachidonic acid metabolites might result predominantly from the activities of the CYP2N proteins in the liver.

In contrast to the similarities between the EETs formed by CYP2N1 and CYP2N2, the CYP2Ns form markedly different HETE products. CYP2N1 forms predominantly 5HETE and also forms 16-, 12-, 9-, and 11-HETEs, in order of decreasing amounts. CYP2N2 forms predominantly 9-HETE, an unidentified HETE, and 20-HETE. Liver microsomes form predominantly 16-HETE and also 19-HETE (Table 8b). In liver, 16HETE could be formed by the action of CYP2N1, but 19-HETE likely is formed by another CYP isoform. This CYP isoform could be CYP2P3 which does form 19-HETE from arachidonic acid and which is expressed in killifish liver (Oleksiak et al., in preparation).

Other fish CYP isoforms appear to be involved in arachidonic acid metabolism in fish livers. Preliminary studies with scup and skate liver microsomes showed that both these fish produce epoxide and hydration products from arachidonic acid (Zacharewski et al., 1996). In addition, later antibody inhibition studies with scup liver microsomes showed that CYP1A was responsible for up to $30 \%$ of liver microsomal arachidonic acid metabolism and that scup CYP2B-like proteins were responsible for up to $43 \%$ of this metabolism (Schlezinger et al., in prep). Thus, CYP other than the CYP2Ns and CYP2P3 likely contribute to the metabolism of arachidonic acid in killifish liver.

Further differences exist between CYP2N1 and CYP2N2 in their rates of metabolism of arachidonic acid, benzphetamine, and ethoxy-, methoxy-, benzyloxy-, and pentoxyresorufin (Table 7). The in vitro rates with expressed CYP may be very different from in vivo rates with a native membrane environment and a natural complement of other proteins such as cytochrome b5 that may affect CYP activities. Nevertheless, in vitro, CYP2N1 metabolizes arachidonic acid more than four times faster than does CYP2N2 and 
more than two times faster than liver microsomes. CYP2N1 and CYP2N2 metabolize benzphetamine with more similar rates which are greater than those of liver microsomes. Neither CYP2N1 nor CYP2N2 has significant O-deethylase activities towards ethoxyresorufin or methoxyresorufin compared to liver microsomes. However, CYP2N1 and CYP2N2 might contribute to liver benzyloxyresorufin O-deethylase activity, and CYP2N1 but not CYP2N2 might contribute to the pentoxyresorufin O-deethylase activity of liver (Table 7).

The results presented here compare the functional properties of two novel CYP, CYP2N1 cloned from liver and CYP2N2 cloned from heart. The CYP2Ns are 75\% identical at the amino acid level. These killifish CYP were classified into a specific CYP family and subfamily based on amino acid sequence similarities with other CYP. This classification convention for CYP proteins ignores functional properties and in doing so, poses difficulties when classifying CYP from more distantly related species. Genetic factors such as different rates of evolution, concerted evolution within a species, codon usage bias, and the occurrance of paralogous genes make the designation of orthologous and paralogous relationships among CYP based solely on sequence similarities difficult, especially in a CYP family as large and diverse as the CYP2 family, which likely contains a number of paralogous genes. Functional characteristics also need to be considered when addressing questions of orthology. Notably, our original hypothesis- that the CYP2Ns might metabolize arachidonic acid because some CYP2Js metabolize arachidonic acid- was mainly based on sequence information (phylogenetic analyses), and this hypothesis was upheld. However, we still cannot distinguish orthologous and paralogous relationships between the CYP2Ns and other CYP2 genes.

The phylogenetic relationship of the CYP2Ns to the CYP2Js and their catalytic similarities suggest that the CYP2Ns are early diverging vertebrate arachidonic acid catylists. The CYP2Ns also are phylogenetically related to the killifish CYP2Ps (Oleksiak 
et al., in preparation). CYP2P3 also metabolizes arachidonic acid (Oleksiak et al., in preparation) but with a very different regio- and stereoselectivity than the CYP2Ns. However, the catalytic similarity with repect to both regio- and stereoselectivity between killifish CYP2P3 and the mammalian CYP2Js, especially CYP2J2, is striking (Oleksiak et al., in preparation). Such conservation in function suggests that arachidonic acid metabolism by cytochromes $\mathrm{P} 450$ is an important feature in non-mammalian vertebrates, and continuing studies in fish should reveal fundamental aspects of arachidonic acid metabolism in vertebrates. High expression of CYP2N2 in heart and brain is especially significant because fishes are ideal organisms in which to study heart and brain development due to their oviparous development and the transparency of their eggs. Little is known about the functions (or even the identities) of CYP in these tissues.

The physiological functions of these CYP2N proteins, especially in heart and brain tissues, are not known but might involve the production of eicosanoids which have biological activities in both heart and brain tissues. Importantly, arachidonic acid epoxygenase products have been shown to be endogenous constituents in both heart and brain tissues in a variety of species (Schlezinger et al., in prep; Junier et al., 1990; Wu et al., 1996; Wu et al., 1997). In addition, scup heart microsomes were shown to metabolize arachidonic acid to epoxy- and hydroxy-metabolites (Schlezinger et al., in prep). High levels of CYP1A protein have been shown in fish heart (Miller et al., 1988), among others, and CYP1A might be contributing to this arachidonic acid metabolism. Another CYP, CYP2J2, recently was identified in heart, and the recombinant CYP2J2 was shown to metabolize arachidonic acid to EET products with chiralities that matched those of the EET enantiomers present in vivo in human heart (Wu et al., 1996). Although a cardiac function has not been ascribed to these EETs, this suggested that CYP2J2 was involved in the epoxidation of endogenous arachidonic acid in heart tissue.

The CYP2Ns form predominantly EET products from arachidonic acid. In the 
heart, the EETs may regulate vascular tone as they do in a number of tissues (Harder et al., 1995). EETs inhibit platelet aggregation (Fitzpatrick et al., 1986), and this might be a general function in the vasculature. The EETs also might have significant effects on cardiac myocytes with respect to excitation-contraction coupling in the heart (Wu et al., 1997). Since the CYP2Ns are similar to the CYP2Js and since both CYP2J2 and CYP2J3 are highly expressed in cardiac myocytes (Zeldin et al., in press), the CYP2Ns likely also are expressed in cardiac myocytes. In addition, the CYP2Ns form 11,12-EET from arachidonic acid and this metabolite has been shown to affect heart contractile function following cardiac ischemia both positively (Wu et al., 1997) and negatively (Moffat et al., 1993).

Cytochrome P450 arachidonic acid metabolites also seem to be important in brain tissue. The concentration of cytochrome $\mathrm{P} 450$ in the brain is about $1 \%$ that of the liver (Warner et al., 1993). Nevertheless, much more cytochrome P450 occurs in the brain of untreated animals than can be accounted for by the hepatic forms, and with the exception of the CYP4As, most P450 in the brains of untreated rats is different from the hepatic forms (Warner et al., 1993). P450s that metabolize arachidonic acid have been found in the hypothalamus (Junier et al., 1990), the pituitary (Capdevila et al., 1984), and the cerebral cortex (Gebremedhin et al., 1992). Importantly, Junier et. al. showed that epoxygenase products of arachidonic acid are endogenous constituents of the rat hypothalamus (Junier et al., 1990).

Both CYP2N1 and CYP2N2 make predominantly 8,9-EET from arachidonic acid, and it is noteworthy that 8,9-EET was the most potent EET (having an EC50 of only $5 \mathrm{pM}$ ) in causing the release of somatostatin from the hypothalamic nerve terminals of the median eminence of rat brain (Junier et al., 1990). This suggests that the CYP2Ns might function in neuropeptide release in fish brain. As well, studies with cat brain suggest vasoactive functions for the arachidonic acid metabolites that the CYP2Ns form. Microsomes from cat 
cerebral cortex formed large amounts of 8,9- and 11,12-EETs and lesser amounts of 14,15-EET (Gebremedhin et al., 1992). Although these products were not quantified, the HPLC profile of arachidonic acid metabolites formed by the cat cerebral microsomes looks remarkably similar to those of the CYP2Ns. Both the cat cerebral microsomes and the recombinant CYP2Ns form predominantly 8,9- and 11,12-EETs. In the cat brain, both 8,9- and 11,12-EET were shown to dilate cerebral arteries through a mechanism that involves activation of calcium-activated potassium channels. Of note, 11(R),12(S)-EET but not $11(\mathrm{~S}), 12(\mathrm{R})$-EET was shown to be a potent vasodilator in renal preglomerular arterioles (Zou et al., 1994). Both CYP2N1 and CYP2N2 form predominantly 11(R),12(S)-EET (Table ), and thus, the CYP2Ns potentially have vasoactivities in fish brain.

The major metabolites that the fish CYP2Ns form from arachidonic acid have been shown to have potent biological effects in heart and brain tissue in a variety of species. CYP2N2 transcripts are highly expressed in killifish heart and brain and CYP2N1 transcripts also are expressed in these tissues. Although message expression does not ensure functional protein expression, this expression pattern suggests that the CYP2Ns might have important functions in the fish heart and brain. These could include the control of the vasculature and vascular functions as well as the control of neuropeptide release.

In summary, we have shown that CYP2N1 and CYP2N2 form a catalytic diversity of arachidonic acid metabolites. These metabolites likely have potent bioactivities in fish, and these fish cytochromes P450, CYP2N1 and CYP2N2, may represent early vertebrate forms of fatty acid epoxygenases and hydroxylases. As CYP from the earliest diverged vertebrate group, they should provide an important link to address the involvement of CYP in endogenous functions that have been conserved in vertebrates. 
CHAPTER 4

IN VITRO EXPRESSION OF A NOVEL CYTOCHROME P450, CYP2P3: AN EARLY VERTEBRATE ARACHIDONIC ACID CATALYST 


\section{$\underline{\text { ABSTRACT }}$}

Multiple genes in two new CYP2 subfamilies (CYP2P and CYP2N) have been identified in the teleost, Fundulus heteroclitus (killifish). Phylogenetically, the mammalian CYP to which these novel CYP2s are related most closely are the CYP2Js (Oleksiak et al., in preparation). The CYP2Ps share similar tissue specific sites of gene transcript expression with the CYP2Js; their expression is restricted to liver and gut just as many of the CYP2Js are expressed highly in these tissues. This suggested that functional similarities also might exist. To test this hypothesis, one of the CYP2P genes, CYP2P3, was co-expressed with cytochrome P450 oxidoreductase in $S f 9$ insect cells using a baculovirus system, and metabolism of arachidonic acid and benzphetamine by CYP2P3 was examined. Expressed CYP2P3 metabolized arachidonic acid with a rate of $135 \mathrm{pmol} / \mathrm{nmol}$ of cytochrome P450/min. The principle reaction products identified by high pressure liquid chromatography and gas chromatography/mass spectroscopy were 14,15epoxyeicosatrienoic acid, 11,12-epoxyeicosatrienoic acid, and 8,9-epoxyeicosatrienoic acid (these EETs comprised $83 \%$ of the total products). 19-hydroxyeicosatetraenoic acid (19HETE) was the only non-epoxide product. CYP2P3 epoxidation of arachidonic acid was highly enantioselective for $14(\mathrm{R}), 15(\mathrm{~S})$-EET (72\% optical purity) and for 8(S),9(R)-EET (76\% optical purity). Hydroxylation of arachidonic acid to 19-HETE also was highly enantioselective with the 19(R)-hydroxyeicosatrienoic acid preferentially formed (78\% optical purity). CYP2P3 also was active in the N-demethylation of benzphetamine with a catalytic turnover of $3.3 \mathrm{nmol} / \mathrm{nmol}$ of cytochrome P450/min. Endogenous EETs and their hydration products, the dihydroxyeicosatrienoic acids (DHETs) were quantified in killifish liver and gut tissues. Amounts of EET regioisomers ranged from 44 to $113 \mathrm{ng} / \mathrm{g}$ tissue and amounts of DHET regioisomers ranged from 16 to $44 \mathrm{ng} / \mathrm{g}$ tissue. The results show that a catalytic diversity of arachidonic acid metabolites occurs in early vertebrates. These 
metabolites formed by CYP2P3 are strikingly similar to those formed by the mammalian CYP2Js, in particular to CYP2J2, suggesting that CYP2P proteins may represent early vertebrate forms of the arachidonic acid catalysts that gave rise to the mammalian CYP2Js.

\section{INTRODUCTION}

Eicosanoids, especially arachidonic acid metabolites, have been identified as regulatory molecules in many vertebrate and invertebrate groups. Arachidonic acid metabolites have been shown to modulate epithelial salt and water transport in mammals (Escalante et al., 1991), frogs, toads (Stanley-Samuelson, 1994), fishes (Wales, 1988) molluscs (Freas and Grollman, 1980) and insects (Petzel and Stanley-Samuelson, 1992). They also have been shown to control various aspects of reproduction in sea stars (Meijer et al., 1986), sea urchins (Schuel et al., 1985), molluscs (Matsutani and Nomura, 1987), insects (Destephano and Brady, 1977), fishes (Wade and Van Der Kraak, 1993), reptiles, and mammals (Liggins et al., 1973). Thus, arachidonic acid metabolites are involved in the regulation of cellular processes that are fundamental to organisms in general, and their synthesis is of primary interest.

The cyclooxygenases, the lipoxygenases, and the cytochromes P450 all metabolize arachidonic acid into eicosanoids (Smith, 1989). Cytochrome P450 proteins can oxygenate arachidonic acid via olefin epoxidation, $\omega$-terminal oxidation, oxidation of allylic or bisallylic carbons, and oxidation with double bond migration (Oliw, 1994). Other polyunsaturated fatty acids such as the $\omega-3$ eicosapentaenoic and docosahexaenoic acids can be similarly oxygenated by cytochromes P450 (VanRollins, 1990). Mammalian cytochrome $\mathrm{P} 450$ arachidonic acid epoxygenases belong predominantly to the CYP2 family while $\omega$-terminal hydroxylations are performed predominantly by cytochromes $\mathrm{P} 450$ in the CYP4 family (Capdevila et al., 1996). In mammals, the biological effects of arachidonic 
acid metabolites have been studied in liver, kidney, cardiovascular tissues, brain, reproductive organs, endocrine tissues, lung, intestine, and platelets (McGiff, 1991). Biological activities include regulation of intracellular calcium concentrations in the liver and kidney, vasoactive effects in numerous tissues, release of peptide hormones in the pituitary, hypothalamus, and pancreas, and anti-aggregatory effects on platelets (McGiff, 1991).

While arachidonic acid appears to have fundamental significance in most animal groups, structural knowledge of the catalysts in its metabolism is largely restricted to mammals. This is particularly true of the cytochrome P450 dependent epoxidation of arachidonic acid. Because cytochrome P450 mediated, arachidonic acid metabolites seem to be important signaling molecules in mammals, they likely have similar critical roles in non-mammalian species. Moreover, some studies have suggested that CYP metabolites of arachidonic acid may be involved in toxicity of chemicals such as $2,3,7,8$ tetrachlorodibenzo- $p$-dioxin (TCDD) and $\beta$-naphthoflavone which alter metabolism of arachidonic acid in some systems (Nakai et al., 1992; Rifkind et al., 1994). However, beyond the few studies in birds, little is known of cytochrome P450 mediated arachidonic acid metabolism in non-mammalian vertebrates, and nothing of the catalysts other than CYP1A (Gilday et al., 1996). In this study, we examined arachidonic acid metabolism by a fish P450 protein. Knowledge of the formation of these metabolites in early diverging vertebrates such as fish could have important implications for our understanding of these processes in vertebrates in general. Conservation of function would suggest a phylogenetically broad, biological importance of a particular pathway while lack of such conservation would suggest an evolutionary diversity of function.

Recently, we identified a number of novel CYP sequences in the fish Fundulus heteroclitus (Oleksiak et al., in preparation). Phylogenetic analysis and tissue expression indicated that these fish CYP were similar to mammalian forms that metabolize arachidonic 
acid. That similarity has prompted an analysis of the functional properties of these fish proteins with arachidonic acid. In this study, the functions of one of these, CYP2P3, is reported. The results reveal a striking similarity between CYP2P3 and CYP2J2 in the arachidonic acid epoxide products each forms. Additionally, the formation of 19-HETE by CYP2P3 as well as by CYP2J3 and CYP2J4 suggests that this hydroxy-metabolite might have physiological importance in vertebrates generally.

\section{MATERIALS AND METHODS}

Materials: $[1-14 \mathrm{C}]$ arachidonic acid was purchased from DuPont NEN. Triphenylphosphine, $\alpha$-bromo-2,3,4,5,6-pentafluorotoluene, N,N-diisopropylethylamine, and diazald were purchased from Aldrich. All other chemicals and reagents were purchased from Sigma unless otherwise specified.

Heterologous Expression of Recombinant CYP2P3: Co-expression of the proteins encoded by the CYP2P3 cDNA and CYPOR (human oxidoreductase) in $S f 9$ insect cells was accomplished using the pAcUW51-CYPOR shuttle vector (kindly provided by Dr. Cosette Serabjit-Singh, Glaxo Research Institute, Research Triangle Park, NC) (Lee et al., 1995) and the BaculoGold Baculovirus Expression System (Pharmingen, San Diego, CA). The CYP2P3 cDNA was cut from the pCNTR cloning vector (5 Prime to 3 Prime) with BamHI and ligated into the pAcUW51-CYPOR linearized with BamHI. In the resulting expression vector (pAcUW51-CYPOR-CYP2P3), the p10 promoter controlled the expression of CYPOR and the polyhedrin vector independently controlled the expression of the CYP2P3. Cultured $S f 9$ insect cells were co-transfected with the transfer vector (pAcUW51-CYPOR-CYP2P3) and linearized wild-type BaculoGold viral DNA in a $\mathrm{CaCl}_{2}$ solution. Recombinant viruses were plaque purified and the presence of the CYP2P3 cDNAs corroborated by PCR analysis. Cultured $S f 9$ cells, grown in spinner flasks at a 
density of 1.5-2.0 X $10^{6}$ cells/ml, were infected with a high titer CYP2P3-CYPOR recombinant viral stock in the presence of $5 \mathrm{uM}$ hemin or $5 \mathrm{uM} \delta$-aminolevulenic acid hydrochloride (Gonzalez, 1991). Cells co-expressing recombinant CYP2P3 and CYPOR were harvested 72 hours after infection, washed twice with phosphate buffered saline, and used to prepare microsomal fractions by differential centrifugation at $4^{\circ} \mathrm{C}$ as described previously (Wu et al., 1996). The P450 content was determined spectrally according to the method of Omura and Sato (Omura and Sato, 1964) using a Shimadzu UV-3000 dual wavelength/double-beam spectrophotometer (Shimadzu Scientific Instruments, Columbia, MD). Microsomes prepared from $S f 9$ insect cells co-expressing CYP2J3 and CYPOR were used as a source of recombinant CYP2J3 (Wu et al., 1997).

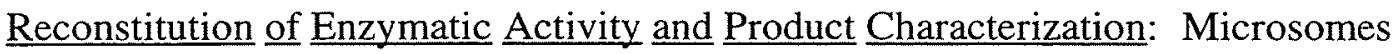
were resuspended to a final reaction volume in $0.05 \mathrm{M}$ Tris- $\mathrm{Cl}$ buffer ( $\mathrm{pH} 7.5$ ) containing $0.15 \mathrm{M} \mathrm{KCl}, 0.01 \mathrm{M} \mathrm{MgCl} 2,8 \mathrm{mM}$ sodium isocitrate, and $0.5 \mathrm{IU}$ of isocitrate dehydrogenase $/ \mathrm{ml}$ and equilibrated at $37^{\circ} \mathrm{C}$ with constant mixing for two minutes before the addition of $\left[1-{ }^{14} \mathrm{C}\right]$ arachidonic acid $(25-55 \mathrm{uCi} / \mathrm{umol}, 70-100 \mathrm{uM}$ final concentration). Reactions were initiated by the addition of NADPH ( $1 \mathrm{mM}$ final concentration) and continued at $37^{\circ} \mathrm{C}$ with constant mixing. Reaction products were extracted into ethyl ether, dried under nitrogen, resolved by reverse-phase HPLC, and quantified by on-line liquid scintillation using a Radiomatic Flo-One B-detector (Radiomatic Instruments, Tampa, FL) (Capdevila et al., 1990). Uninfected Sf9 cells and reactions without NADPH addition were used as negative controls. Individual EETs were identified by comparing their reverse-phase and normal phase HPLC properties with those of authentic standards and by GC/MS analysis (Capdevila et al., 1986) (Capdevila et al., 1990; Clare et al., 1991). For chiral analysis, the EETs were collected batchwise from the HPLC eluent, derivatized to the corresponding EET-PFB or EET-methyl esters, purified by normal-phase HPLC, resolved into the corresponding antipodes by chiral-phase HPLC, and quantified 
by liquid scintillation as described previously (Capdevila et al., 1991) (Hammonds et al., 1989). 19-HETE was identified based on co-elution with authentic standard on reverseand normal-phase HPLC and by GC/MS analysis (Capdevila et al., 1982; Capdevila et al., 1985; Falck et al., 1990). 19-HETE chiral analyses were performed on naphthylated 19HETE methyl esters using a Pirkle covalent D-phenyl glycine column (5um, 4.6x250 mm, Regis Chemical Company, Morton Grove, IL) equilibrated with hexane $99.75 \%$ /isopropanol $0.25 \%$ at $1 \mathrm{ml} / \mathrm{min}$ (Oliw, 1990). Under the conditions, the retention times for 19(R)- and 19(S)-HETE are $76 \mathrm{~min}$ and $80 \mathrm{~min}$, respectively, with a resolution factor of 0.93 .

Quantification of Endogenous EETs and DHETs in Killifish Liver and Gut: Methods used to quantify endogenous EETs and DHETs present in fish liver and gut tissues were similar to those used to quantify EETs in human heart (Wu et al., 1996). Tissues frozen in liquid nitrogen were homogenized in $15 \mathrm{ml}$ of phosphate buffered saline containing triphenylphosphine $(5-10 \mathrm{mg}$.). The homogenate was extracted twice under acidic conditions with two volumes of chloroform/methanol (2:1) and once more with an equal volume of chloroform. The combined organic phases were evaporated in tubes containing mixtures of $\left[1-{ }^{14} \mathrm{C}\right] 8,9-, 11,12-, 14,15$-EET or $[1-14 \mathrm{C}] 8,9-, 11,12-, 14,15-$ DHET internal standards $(55-57 \mathrm{uCi} / \mathrm{umol}, 30 \mathrm{ng}$, each). Saponification to recover phospholipid bound EETs or DHETs was followed by silica column purification. The eluent, containing a mixture of radiolabeled internal standards and total endogenous EETs/DHETs, was resolved into individual regioisomers by HPLC as described (Karara et al., 1989; Capdevila et al., 1991). For analysis, aliquots of individual EET-PFBs or DHET-PFB-trimethylsilyl (TMS) ethers were dissolved in dodecane and analyzed by GC/MS on a Kratos Concept ISQ mass spectrometer (Kratos Analytical, Inc., Ramsey, $\mathrm{NJ})$ operating under negative ion chemical ionization conditions, at $5.3 \mathrm{keV}$ accelerating potential, at a mass resolution of 1200 and using methane as a bath gas. EET 
quantifications were made by selected ion monitoring at $\mathrm{m} / \mathrm{z} 319$ (loss of PFB from endogenous EET-PFB and $\mathrm{m} / \mathrm{z} 321$ (loss of PFB from [1-14C]EET-PFB standards). The 5,6-EET was not quantified due to its inherent instability. DHET quantifications were made by selected ion monitoring at $\mathrm{m} / \mathrm{z} 481$ (loss of PFB from endogenous DHET-PFBTMS) and $m / z 483$ (loss of PFB from [1-14 C]DHET-PFB-TMS internal standards). The EET-PFB/[1-14C]EET-PFB and DHET-PFB/[1-14C]DHET-PFB ratios were calculated from the integrated values of the corresponding ion current intensities.

Benzphetamine $\mathrm{N}$-demethylase activity: The benzphetamine $\mathrm{N}$-demethylase activity of the recombinant CYP2P3 was assessed using the same microsomal preparations used for arachidonic acid assays under identical reaction conditions but employing benzphetamine ( $2 \mathrm{mM}$ final concentration) as the substrate. The reaction product (formaldehyde) was quantified spectrophotometrically according to the method of Nash (Nash, 1953).

O-deethylase activities: The ethoxyresorufin, pentoxyresorufin, methoxyresorufin and benzyloxyresorufin O-deethylase activities of the recombinant CYP were assessed using the same microsomal preparations used for arachidonic acid and benzphetamine assays. The resorufin reaction products were quantified using the cytofluor method described by Hahn et al. (Hahn et al., 1993).

Synthetic Procedures: The $\left[1-{ }^{14} \mathrm{C}\right] \mathrm{EET}$ internal standards were synthesized from $\left[1-{ }^{14} \mathrm{C}\right]$ arachidonic acid $(55-57 \mathrm{uCi} / \mathrm{umol})$ by nonselective epoxidation as described (Falck et al., 1990). Racemic and enantiometrically pure EETs were prepared by total chemical synthesis according to published procedures (Corey et al., 1980; Falck and Manna, 1982; Mosset et al., 1986; Moustakis et al., 1986). Methylations were performed using an ethereal solution of diazomethane (Capdevila et al., 1986). PFB esters were formed by reaction with pentafluorobenzyl bromide as described (Karara et al., 1989). TMS ethers were prepared using $25 \%$ (v/v) bis(trimethylsilyl)trifluoracetamide in anhydrous pyridine 
(Porter et al., 1979). HETE standards were purchased from Cayman Chemical Company. Naphthoyl esters were prepared by adding 1M 1-naphthoyl chloride dissolved in anhydrous toluene to 19-HETE samples dissolved in anhydrous pyridine. Omega terminal alcohols of arachidonic acid were synthesized as described (Manna et al., 1983)

Statistical Methods: All values are expressed as mean $+/$-standard error (SEM) or standard deviation of the mean.

\section{RESULTS}

Metabolism of Arachidonic Acid by Recombinant CYP2P3: The Fundulus heteroclitus CYP2P3 protein was co-expressed with CYPOR in SF9 insect cells using the baculovirus expression system according to previously described methods (Wu et al., 1996; Lee et al., 1995; Gonzalez et al., 1991). The level of expression of recombinant CYP2P3 was 3-10 nmol P450/liter of infected SF9 cells. Recombinant CYP2P3 showed a typical CYP Soret absorption at $\sim 450 \mathrm{~nm}$ when reduced and complexed with carbon monoxide (Figure 1).

To examine the catalytic properties of the recombinant hemoprotein towards arachidonic acid, we incubated microsomal fractions containing both the CYPOR and CYP2P3 with arachidonic acid in the presence of NADPH and an NADPH regenerating system. The total rate of metabolism was $135 \mathrm{pmol}$ of product formed $/ \mathrm{nmol} \mathrm{P} 450 / \mathrm{min}$ at $37^{\circ} \mathrm{C}$. As shown in Figure 2, CYP2P3 metabolized arachidonic acid to 14,15-, 11,12- and 8,9-EETs as the major reaction products and to 19-HETE. The reverse- and normal-phase HPLC (Figures 2 and3) properties of the HETE product formed by CYP2P3 identify it as 19-HETE. The EETs accounted for $83 \%$ of the total reaction products. Each of the major 


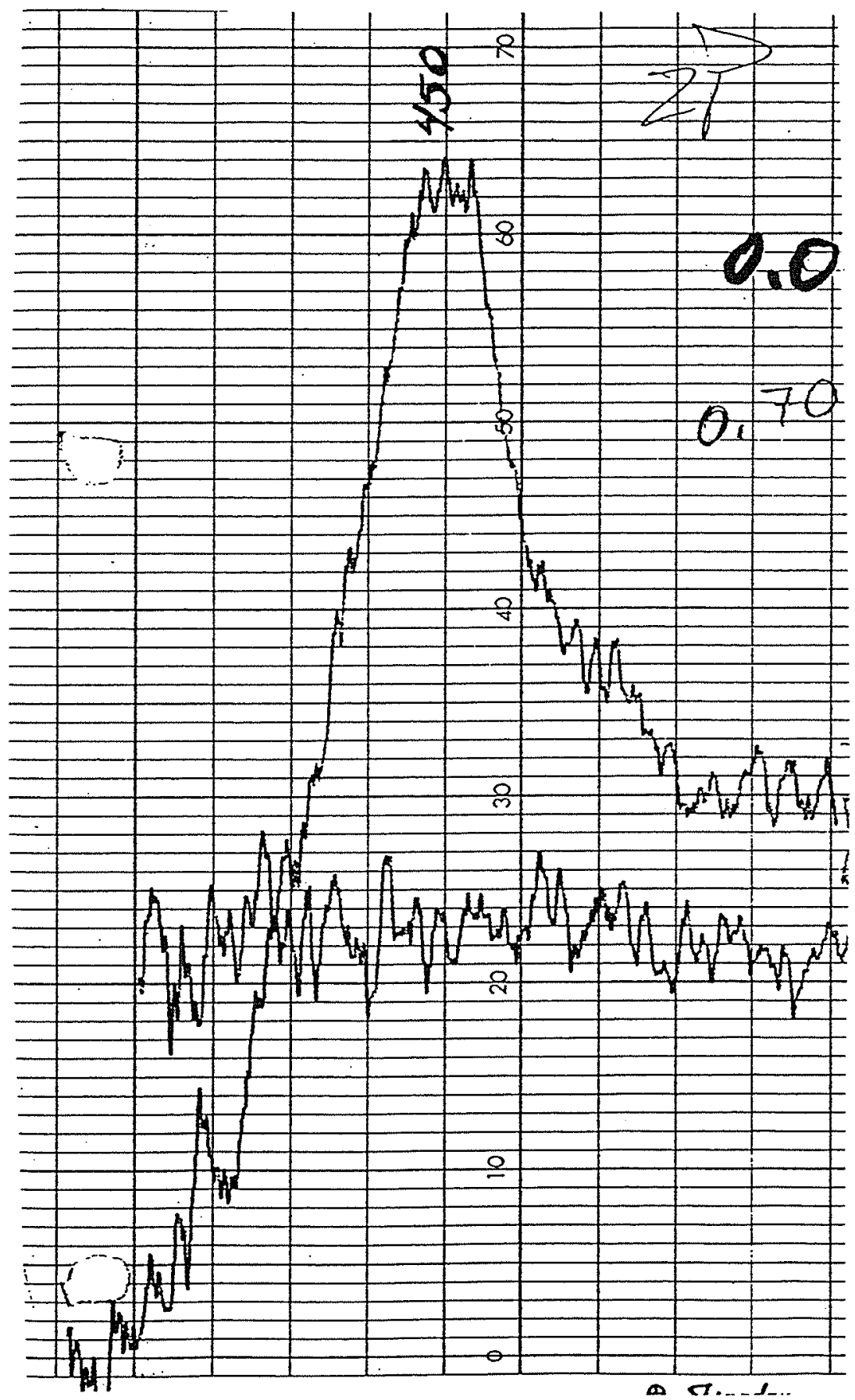

Figure 1. Spectrophotometric analysis of CYP2P3 expressed in Sf9 microsomes. P450 content was analyzed by measuring the dithionite difference spectrum in CO-treated microsomes. 
products was identified by comparing their reverse- and normal-phase HPLC properties with those of authentic standards and by GC/MS. None of these metabolites were formed in the absence of NADPH showing that the reaction was P450 mediated (Figure 2).

The chromatogram in Figure 2 demonstrates that CYP2P3 is both an arachidonic epoxygenase and $\omega-1$ hydroxylase. Regiochemical analysis of the EETs revealed a slight preference for epoxidation at the 14,15 -olefin which made up $36 \%$ of the total EET products (Table 1) while epoxidation at the 11,12- and 8,9-olefins represented 33\% and $31 \%$ of total EET production, respectively. Epoxidation at the 5,6-olefin did not occur. Stereochemical analysis of the CYP2P3-derived EETS revealed a high preference for 14(R),15(S)- and 8(S),9(R)-EETs (optical purities 72\% and 76\%, respectively) (Table 1). In contrast, the 11,12-EET was formed as a nearly racemic mixture (Table 1). 19-HETE was the only HETE formed and comprised $17 \%$ of the total CYP2P3 products. Stereochemical analysis of the 19-HETE revealed a high preference for 19(R)-HETE (optical purity: 78\%) (Table 1). By comparison, chiral analysis of the 19-HETE formed during incubations of recombinant CYP2J3 with arachidonic acid revealed a strikingly similar preference for 19(R)-HETE (optical purity: 70\%).

Endogenous EETs and DHETs: Analysis of endogenous EETs and DHETs present in killifish liver and gut tissues revealed that both tissues contain comparable amounts of these eicosanoids (Figures 4 and 5). Fish liver contained $\sim 210 \mathrm{ng} \mathrm{EETs/g} \mathrm{tissue} \mathrm{and}$ intestine contained $~ 200 \mathrm{ng}$ EETs/g tissue (Figure 4). 14,15-EET was the predominant regioisomer recovered from both liver and gut comprising $52 \%$ and $47 \%$ of the total EETs, respectively. Both 11,12- and 8,9-EET were recovered in lower amounts from these tissues (Figure 4). Fish liver and gut contained $\sim 70$ and 75 ng DHET/g tissue, respectively. In contrast to the EETs, the three DHETs were recovered in nearly equal amounts from both liver and gut (Figure 5). 

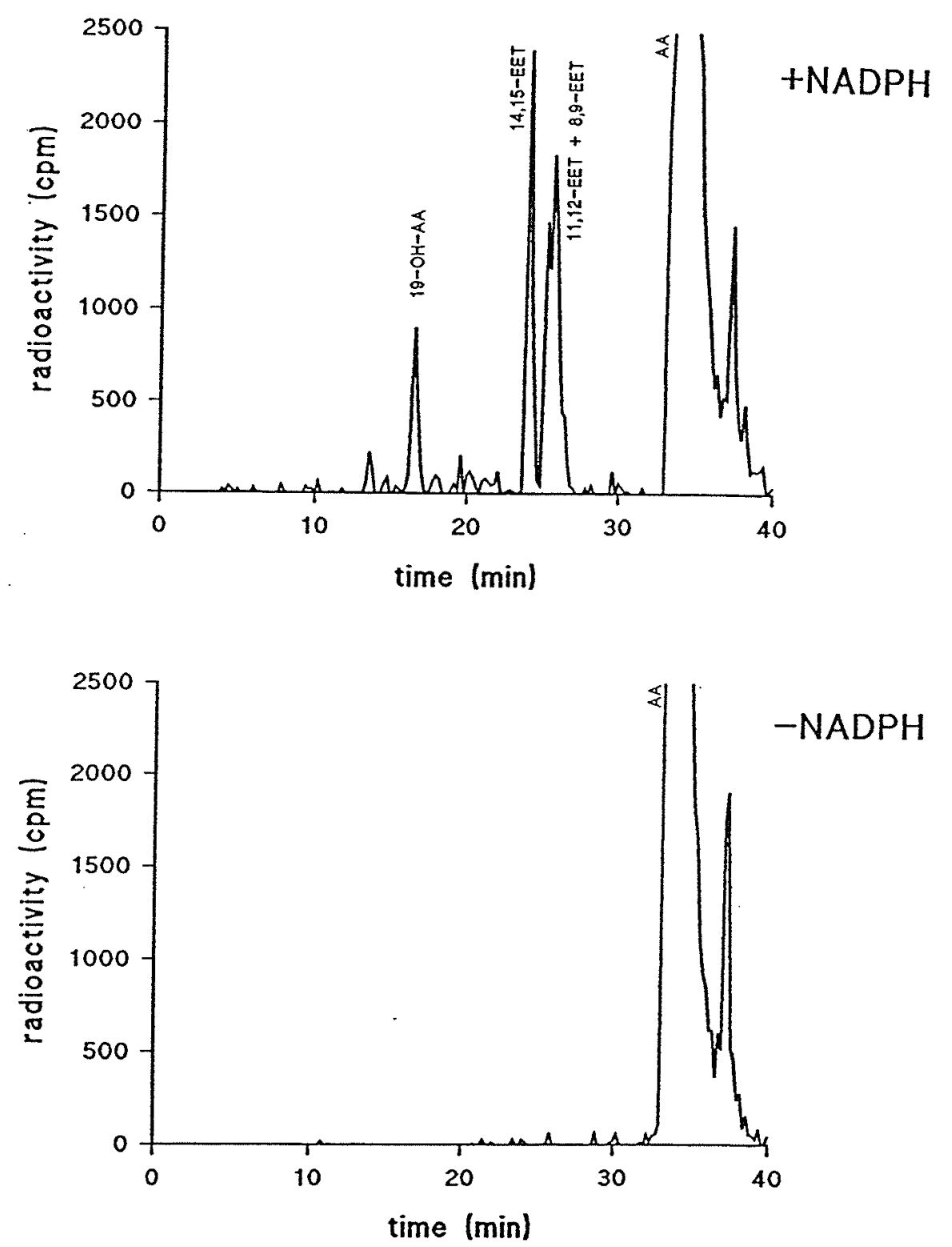

Fig. 2. Reverse-phase HPLC chromatogram of the organic soluble metabolites generated during incubation of recombinant CYP2P3 with $\left[1-1^{4} \mathrm{C}\right]$ arachidonic acid. Microsomal fractions prepared from CYP2P3/CYPOR transfected $S f 9$ insect cells were incubated at $37^{\circ}$ $\mathrm{C}$ with [1-14 C]arachidonic acid (70-100 uM, final concentration) with/without NADPH (1 $\mathrm{mM}$, final concentration) and an NADPH regenerating system. After 1 hour, the reaction products were extracted and resolved by reversed-phase HPLC as described. Peak identifications were made by comparisons of the HPLC properties of individual peaks with those of authentic standards using both reverse-phase and normal-phase HPLC and by GC/MS. Ordinate: radioactivity in cpm; abscissa: time in min. Left panel: incubation with NADPH; right panel: incubation without NADPH. 

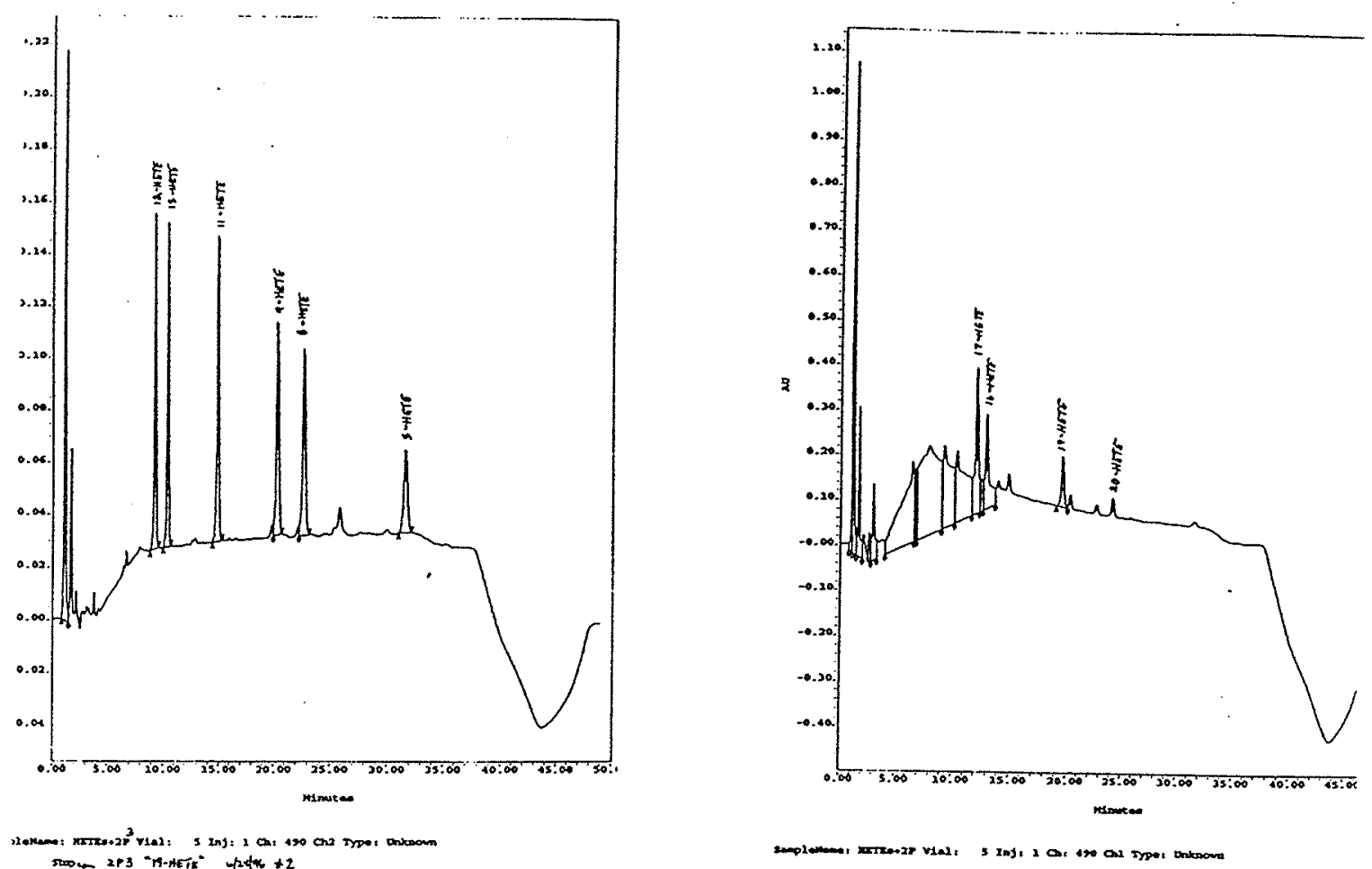

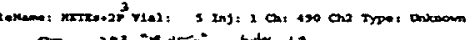

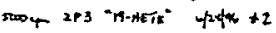

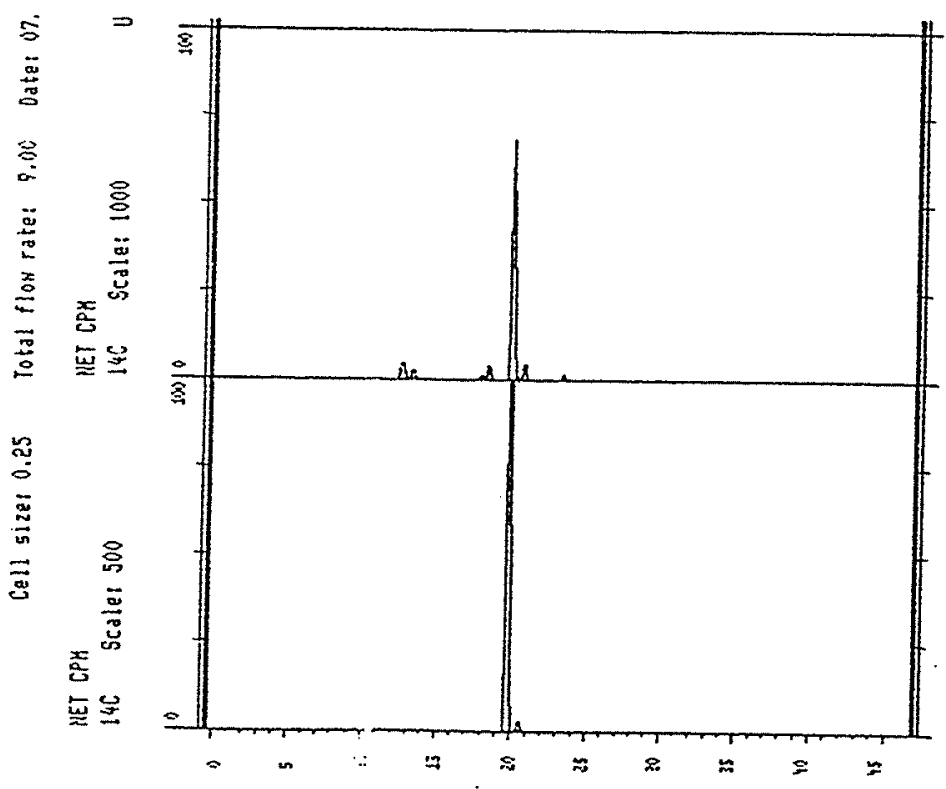

Figure 3. Normal-phase HPLC chromatogram of the HETE metabolite generated during incubation of recombinant $\mathrm{CYP} 2 \mathrm{P} 3$ with $[1-14 \mathrm{C}]$ arachidonic acid co-injected with authentic HETE standards. Top panel: 12-, 15-, 11-, 9-, 8-, and 5-HETE standards. Ordinate: absorbance units at $234 \mathrm{~nm}$; abscissa: time in minutes. Middle panel: 17-, 16-, 19-, and 20-HETE standards. Ordinate: absorbance units at $234 \mathrm{~nm}$; abscissa: time in minutes. Bottom panel: HETE sample. Ordinate: radioactivity in cpm; abscissa: time in minutes. 
Table 1.

Regio- and stereochemical composition of EETs and 19-HETE produced by recombinant CYP2P3.

\begin{tabular}{lccccc}
\hline Regioisomer & \multicolumn{2}{c}{ Distribution } & \multicolumn{2}{c}{ Enantioselectivity } \\
& $\%$ & (\% total EETs) & R,S & S,R \\
\hline 14,15-EET & 30 & $(36)$ & 72 & 28 \\
11,12-EET & 27 & $(33)$ & 48 & 52 \\
$8,9-$ EET & 26 & $(31)$ & 24 & 76 \\
& & & $19(\mathrm{R})$ & $19(\mathrm{~S})$ \\
\hline 19-HETE & 17 & & 78 & 22 \\
& & & & \\
\hline
\end{tabular}

The activity of recombinant CYP2P3 was reconstituted in the presence of NADPH and an NADPH regenerating system as described in Materials and Methods. After one hour, the EET products were extracted into ethyl ether, resolved into individual regioisomers by reverse and normal-phase HPLC, derivatized to corresponding EET-PFB or EET-methyl esters, purified by normal-phase HPLC, and resolved into the corresponding antipodes by chiral -phase HPLC. 19-HETE was similarly resolved. Values shown are averages of at least three different experiments with $\mathrm{SEM}<5 \%$. 
Figure 4. Endogenous EETs recovered from liver and gut tissues (ng/g tissue).

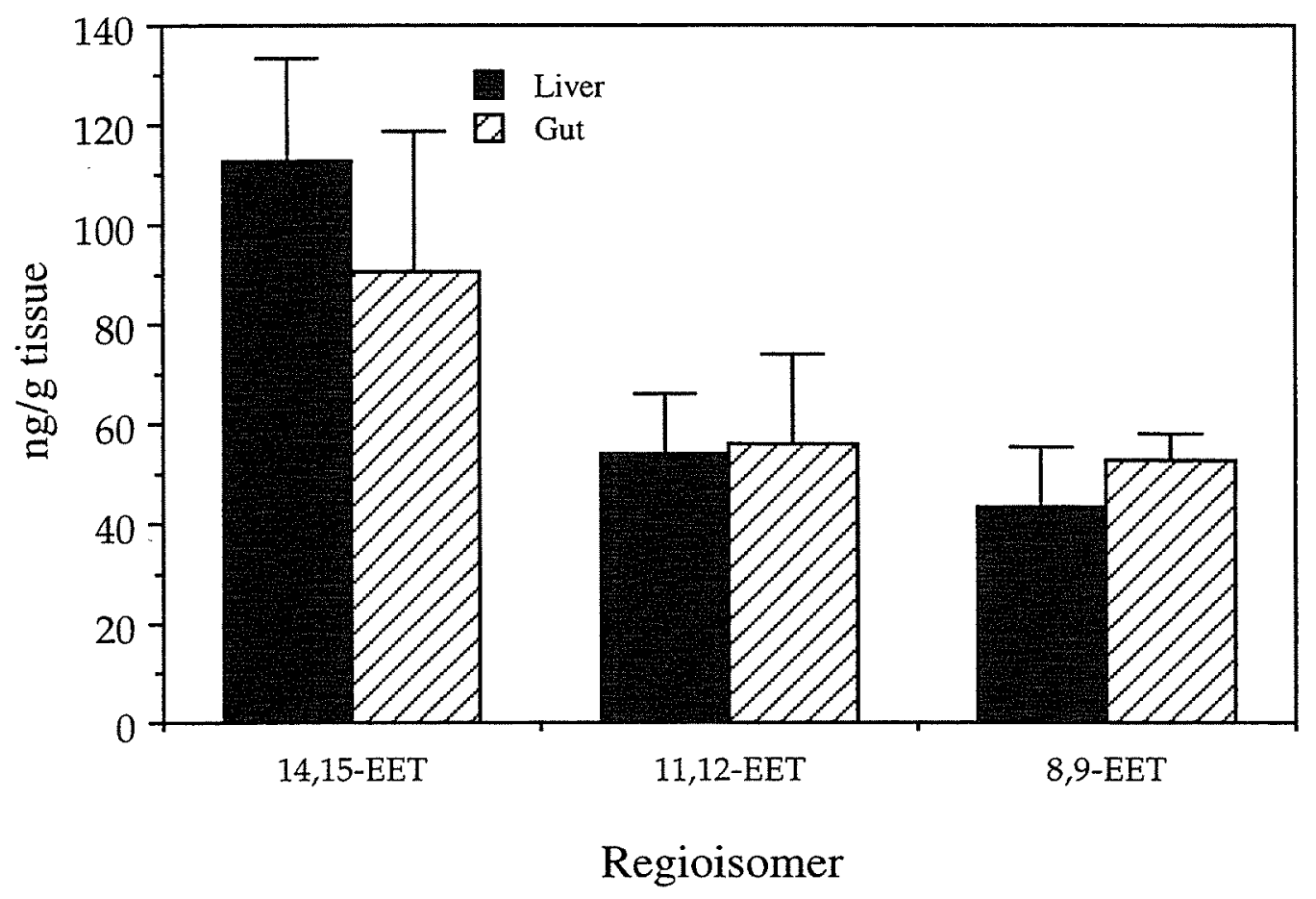

Levels of the different EET regioisomers were quantified as described in Materials and Methods. Values shown are means of three individuals with standard deviations. 
Figure 5. Endogenous DHETs recovered from liver and gut tissues (ng/g tissue).

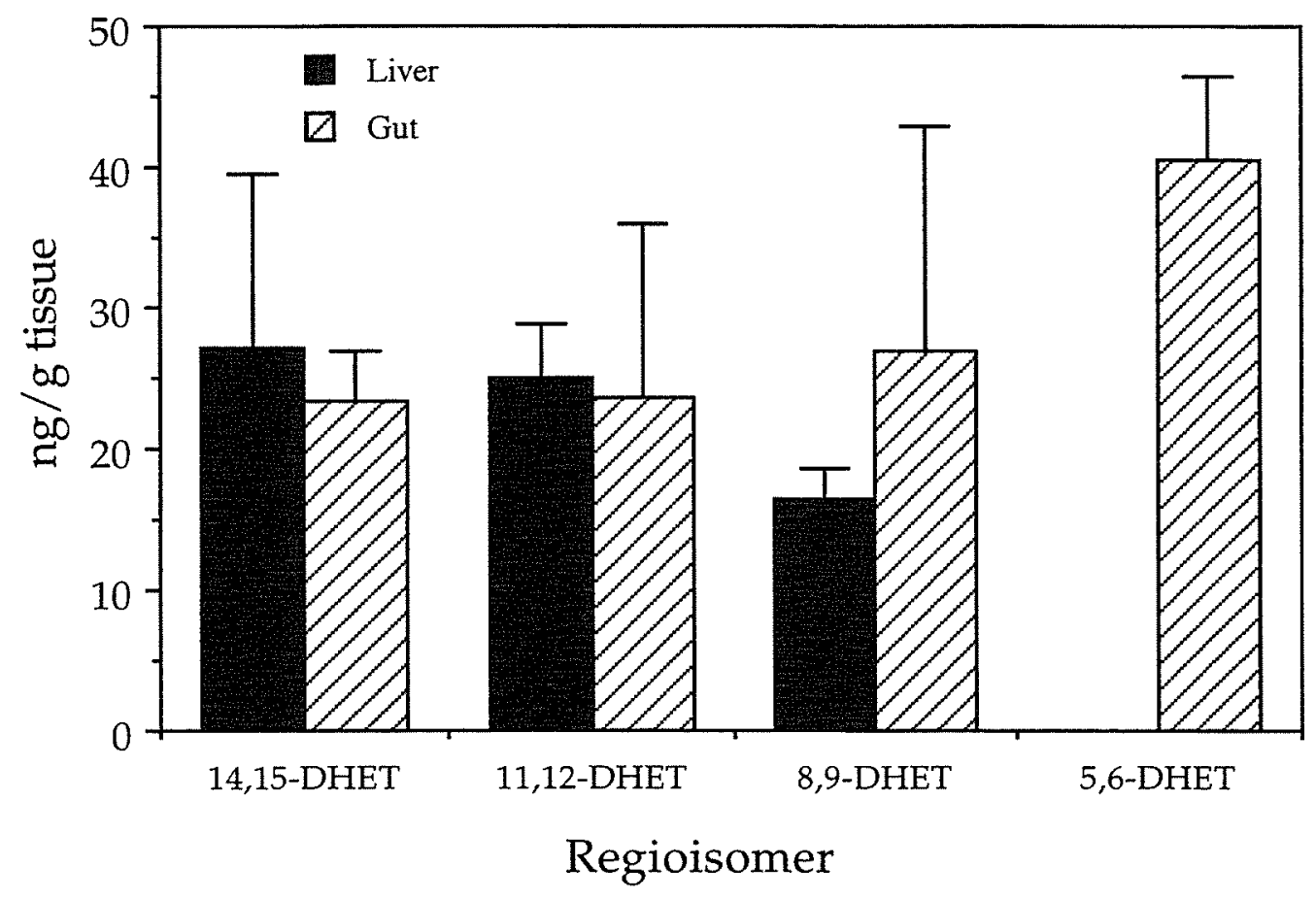

Levels of the different DHET regioisomers were quantified as described in materials and methods. Values shown are means of three individuals with standard deviations. 


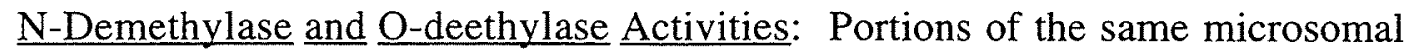
preparations used for arachidonic acid studies were used to investigate the $\mathrm{N}$-demethylase activity of CYP2P3 towards benzphetamine as well as its O-deethylase activities towards ethoxyresorufin, pentoxyresorufin, benzyloxyresorufin, and methoxyresorufin. CYP2P3 had substantial benzphetamine $\mathrm{N}$-demethylase activity of $3.3 \mathrm{nmol} / \mathrm{nmol} \mathrm{CYP} / \mathrm{min}$ (Table 2). In contrast, relative to activities of killifish liver microsomes (Oleksiak et al., in preparation), CYP2P3 had insignificant ethoxy-,pentoxy-, benzyloxy-, and methoxy-Odeethylase activities (Table 2).

\section{DISCUSSION}

A major difficulty in discerning the endogenous function or substrate of a particular CYP is that the catalytic function of these proteins can be strongly affected by minor structural changes. Thus, change in only one amino acid can dramatically alter the substrate specificity of the CYP (Lindberg and Negishi, 1989). This is particularly a problem in a gene family as large as the CYP2 family due to the possibility of concerted evolution. Concerted evolution results from homologous recombination between similar sequences and is common in multigene families (Hood et al., 1975). In a large, closely related subset of the CYP superfamily of sequences, such as the CYP2 family, concerted evolution might mask functional relationships derived from phylogenetic relationships. One approach to identifying endogenous functions is to evaluate the functional relationship of structurally homologous CYP in different taxonomic groups. In this study we examined the function of a recently identified CYP2P that had been identified as being related to the CYP2Js, based on both phylogeny and tissue distribution (Oleksiak et al., in preparation). In order to further examine the relationships between the CYP2Ps and the CYP2Js, one of the CYP2Ps, CYP2P3, was co-expressed with cytochrome P450 oxidoreductase in $S f 9$ 
Table 2.

Metabolic rates (pmol/min/nmol CYP) of recombinant CYP2P3 incubated with different substrates.

\begin{tabular}{llllll}
\hline AA & Benz. & ER & MR & BR & PR \\
\hline & & & & & \\
135 & 3300 & 2 & 2 & ND & ND \\
\hline
\end{tabular}

AA: arachidonic acid; Benz.: benzphetamine; ER: ethoxyresorufin; MR: methoxyresorufin; BR: benzyloxyresorufin; PR: pentoxyresorufin; ND: not detected. The activities of recombinant CYP2P3 was reconstituted in the presence of NADPH and an NADPH regenerating system as described in Materials and Methods. Values shown are means of at least three different experiments with $\mathrm{SEM}<15 \%$. 
insect cells using a baculovirus system, and its ability to carry out regio- and stereospecific metabolism of arachidonic acid was examined.

We found that CYP2P3 is active in the metabolism of arachidonic acid to EETs and 19-HETE; hence, CYP2P3 is both an arachidonic acid epoxygenase and an arachidonic acid $\omega-1$ hydroxylase. Analysis of the regioisomer distribution and enantioselectivities of the CYP2P3-derived eicosanoid products revealed striking similarities to mammalian CYP2J products (Wu et al., 1996; Wu et al., 1997; Zhang et al., 1997). For example, both fish CYP2P3 and human CYP2J2 form predominantly arachidonic acid epoxides. In both cases, 14,15-EET is the primary epoxygenase product formed followed by lower amounts of the other EET regioisomers (Table 3). CYP2P3 and CYP2J2 also generate arachidonic acid epoxides that share similar enantioselective properties. Thus, both enzymes favor epoxidation at the re, si face of the 14,15-olefin but produce racemic 11,12EET (Table 3). However, unlike CYP2J2, CYP2P3 is highly selective for $8(\mathrm{~S}), 9(\mathrm{R})$ EET (76\%) (Table 3). The stereospecificity of CYP2P3 at the 8,9 position suggests that the substrate binding site for CYP2P3 might be more constrained than that of CYP2J2 which lacks this specificity. Determining the structural differences between the substrate binding sites of CYP2P3 and CYP2J2 might elucidate the basis for these functional differences.

A distinct similarity between CYP2P3 and the CYP2Js is the formation of the 19HETE metabolite. Other CYP also form 19-HETE from arachidonic acid. 19-HETE is a major reaction product of rat CYP1A1 (Falck et al., 1990), and both human (Rifkind et al., 1995) and rabbit (Laethem et al., 1992) CYP2E1 generate substantial amounts of 19-HETE from arachidonic acid. In rabbit, 19-HETE comprised $46 \%$ of the total metabolites produced and was 70\% 19(S)-HETE and 30\% 19-(R)-HETE (Laethem et al., 1993). Both 19(R) and (S)-HETE were shown to be vasoactive, and 19(S)-HETE also was shown to be a specific stimulator of the renal cortical $\mathrm{Na}^{+} \mathrm{K}^{+}$-ATPase (Escalante et al., 1988). 19- 
Table 3.

Comparison of the distribution and stereochemistry of the EETs produced by CYP2P3 to the same regioisomers produced by CYP2J2.

\begin{tabular}{lccc}
\hline & \multicolumn{3}{c}{ Distribution (\%) } \\
CYP & 14,15 -EET & 11,12 -EET & 8,9 -EET \\
\hline 2P3 & 30 & 27 & 26 \\
$2 \mathrm{~J} 2$ & 37 & 18 & 24 \\
\hline
\end{tabular}

\begin{tabular}{|c|c|c|c|c|c|c|}
\hline \multirow[b]{2}{*}{ CYP } & \multicolumn{6}{|c|}{ Enantioselectivity } \\
\hline & $\begin{array}{c}\% 14(\mathrm{R}) \\
15(\mathrm{~S}) \\
\end{array}$ & $\begin{array}{c}\% 14(\mathrm{~S}) \\
15(\mathrm{R}) \\
\end{array}$ & $\begin{array}{r}\% 11(\mathrm{R}) \\
12(\mathrm{~S}) \\
\end{array}$ & $\begin{array}{r}\% 11(\mathrm{~S}) \\
12(\mathrm{R})\end{array}$ & $\begin{array}{r}\% 8(\mathrm{R}) \\
9(\mathrm{~S}) \\
\end{array}$ & $\begin{array}{r}\% 8(\mathrm{~S}) \\
9(\mathrm{R}) \\
\end{array}$ \\
\hline $2 \mathrm{P} 3$ & 72 & 28 & 48 & 52 & 24 & 76 \\
\hline $2 \mathrm{~J} 2$ & 76 & 24 & 49 & 51 & 47 & 53 \\
\hline
\end{tabular}


HETE is the only HETE formed by CYP2P3 and is the principle product formed by both CYP2J3 and CYP2J4 (Wu et al., 1996; Wu et al., 1997; Zhang et al., 1997). This suggests that 19-HETE might be an important metabolic product. CYP2P3, CYP2J3 and CYP2J4 all are expressed in gut tissue. Interestingly, Macica et al. found that 19-HETE made up 33\% of the products of arachidonic acid metabolism in rabbit intestine and found that 19(R)-HETE induced mesenteric dilation (Macica et al., 1993). CYP2P3 produces predominantly 19(R)-HETE as does CYP2J3 (Darryl Zeldin, personal communication) providing further evidence that these heme thiolate proteins are quite similar from a catalytic standpoint. The similar site of expression and the similar stereospecificity for this metabolite suggest that the 19(R)-HETE metabolite formed by CYP2P3, CYP2J3, and CYP2J4 might have a physiological function in gut, among other tissues. However, although the regio- and stereoselectivities shown by CYP2P3 and the CYP2Js could reflect an important function for the regio- and stereoisomers formed in greatest abundance, the CYP2P3 and CYP2J proteins also could be constrained by the need not to form high quantities of the less abundant metabolites. Thus, although the 19(S)-HETE product is formed with only $22 \%$ stereoselectivity by CYP2P3, it might be the physiologically important metabolite.

The metabolic rate of expressed CYP2P3 for arachidonic acid is $135 \mathrm{pmol} / \mathrm{nmol}$ cytochrome P450/min. which is in the range of rates of expressed CYP2J2, CYP2J3 and CYP2J4 which are 65,200 , and $150 \mathrm{pmol} / \mathrm{nmol}$ cytochrome P450/min., respectively (Wu et al., 1996; Wu et al., 1997; Zhang et al., 1997). Fish CYP2P3 and the mammalian CYP2Js also exhibit similar specificities with respect to xenobiotic substrates. CYP2P3, CYP2J1, and CYP2J3 all metabolize benzphetamine at appreciable rates $(3.3,3.9$, and 3.0 nmol/nmol cytochrome P450/min., respectively). Neither CYP2P3 nor CYP2J3 significantly metabolizes ethoxyresorufin, pentoxyresorufin, methoxyresorufin, or benzyloxyresorufin (Table 2) (Oleksiak, Zeldin, and Stegeman, unpublished). While these 
in vitro rates may be different from those in vivo with a native membrane environment and accessory proteins such as cytochrome b5 that could affect rates, these rates do suggest a strong catalytic similarity between CYP2P3 and CYP2J2, CYP2J3, and CYP2J4.

The similarities between CYP2P3, CYP2J2, CYP2J3, and CYP2J4 contrast with the differences between CYP2P3 and the CYP2Ns (Table 4). The CYP2Ns (CYP2N1 and CYP2N2, also isolated from killifish) also share similar sequences and tissue distributions with the CYP2Ps and the CYP2Js (Oleksiak et al., in preparation). In particular, CYP2N1 is expressed predominantly in liver, gut. The CYP2Ns also metabolize arachidonic acid, but the metabolites they form from arachidonic acid are very different from those formed by CYP2P3 and the CYP2Js (Table 4). In contrast to CYP2P3, both CYP2N1 and CYP2N2 form predominantly 8,9-EET with very high regioselectivities $(91 \%$ and $90 \% 8(\mathrm{R}), 9(\mathrm{~S})$ EET, respectively). They form 11,12-EET as the next most abundant epoxide with CYP2N1 having 92\% selectivity for $11(\mathrm{R}), 12(\mathrm{~S})$-EET and CYP2N2 having $70 \%$ stereoselectivity. Both CYP2N proteins form 14,15-EET in the least amount for which CYP2N1 shows little selectivity, and CYP2N2 shows $68 \%$ selectivity for $14(\mathrm{~S}), 15(\mathrm{R})$ EET. Most strikingly, neither CYP2N1 nor CYP2N2 forms 19-HETE. Instead they form a variety of different HETEs; CYP2N1 forms 5-, 16-, 12-, 9-, and 11-HETE in order of decreasing amounts while CYP2N2 forms 9-, an unidentified-, and 20-HETE also in order of decreasing amount. These differences might reflect very different physiological roles for the CYP2P and the CYP2N proteins. Moreover, the presence of multiple arachidonic acid catalysts in killifish highlights the potential importance of arachidonic acid metabolites in early diverging vertebrates.

The demonstration of EETs and their corresponding hydration products (the DHETs) as endogenous constituents of killifish liver and gut tissues suggest that arachidonic acid epoxidation and EET hydration occur in vivo in these tissues. Liver and gut contain similar amounts of EET and DHET regioisomers (Figures 4 and 5). Amounts 
Table 4.

Comparison of the distribution and stereochemistry of the EETs and HETEs produced by CYP2P3 to the regioisomers produced by CYP2N1 and CYP2N2.

\begin{tabular}{lcccc}
\hline & \multicolumn{4}{c}{ Distribution (\%) } \\
CYP & 14,15 -EET & 11,12 -EET & 8 -9-EET & इHETE \\
\hline $2 \mathrm{P} 3$ & 30 & 27 & 26 & 17 \\
$2 \mathrm{~N} 1$ & 5 & 17 & 38 & 40 \\
$2 \mathrm{~N} 2$ & 8 & 9 & 38 & 45 \\
& & & & \\
\hline
\end{tabular}

\begin{tabular}{lcccccc}
\hline & \multicolumn{7}{c}{ Enantioselectivity } \\
CYP & $\% 14(\mathrm{R})$ & $\% 14(\mathrm{~S})$ & $\% 11(\mathrm{R})$ & $\% 11(\mathrm{~S})$ & $\% 8(\mathrm{R})$ & $\% 8(\mathrm{~S})$ \\
$2 \mathrm{P} 3$ & 72 & 28 & 48 & 52 & 24 & 76 \\
$2 \mathrm{~N} 1$ & 49 & 51 & 92 & 8 & 91 & 9 \\
$2 \mathrm{~N} 2$ & 32 & 68 & 70 & 30 & 90 & 10 \\
\hline
\end{tabular}

$\Sigma$ HETE was 19-HETE for CYP2P3, 5-, 16-, 12-, 9-, and 11-HETE in order of decreasing amounts, for CYP2N1, and 9-, an unidentified-, and 20-HETE also in order of decreasing amounts, for CYP2N2. 
of different EET regioisomers ranged from 44 to $113 \mathrm{ng} / \mathrm{g}$ tissue and those of the DHET regioisomers ranged from 16 to $44 \mathrm{ng} / \mathrm{g}$ tissue. Since all of these fish had been feeding on the same diet for more than a year, the variability between fish in the levels of EETs and DHETs may reflect some unknown type of regulation of arachidonic acid and/or its metabolites. The levels of epoxygenase products detected in killifish liver are lower than those found in mammalian liver tissues. This may reflect the lower arachidonic acid availability in marine organisms as compared with terrestrial organisms (Zabelinskii et al., 1995). Interestingly, killifish gut had a 5-fold higher EET content than human gut (Zeldin et al., In press) suggesting the presence of an active arachidonic acid epoxygenase(s) in fish intestine. CYP2P3 transcript is highly expressed in gut and liver and the regiochemical profile of endogenous killifish EETs matches the regiochemistry of expressed CYP2P3 products suggesting that $\mathrm{CYP} 2 \mathrm{P} 3$ may contribute significantly to the epoxidation of arachidonic acid in these tissues. Interestingly, the stereochemistry of EETs recovered from human jejunum showed that 14(R),15(S)-, 11(R),12(S)-, and 8(S),9(R)-EETs were the predominant antipodes (Zeldin et al., 1997), and CYP2P3 also forms predominantly 14(R),15(S)- and 8(S),9(R)-EETs. These epoxides that have been reincorporated into the plasma membrane might have an endogenous physiological function. Incorporation of EETs into cellular lipids has been shown to affect the physiochemical properties and thus the structural and functional properties of many biological membranes (Capdevila et al., 1993). However, they might be "left-over" metabolites that are not used and instead are dumped back into the membrane (i.e. the 19-HETE metabolite might be the only physiologically relevant arachidonic acid metabolite). Alternatively, they might be physiologically important metabolites that are stored in the membrane.

The EETs, HETEs, and DHETs have been shown to possess important biological activities in mammals. In general, the EETs have potent vasoactivities in a variety of tissues (Harder et al., 1995). This might be an important function in terms of gut 
circulation. Notably, EETs have been shown to be potent vasodilators in the intestinal microcirculation (Proctor et al., 1987). The 19-HETE product also might function in the gut. As mentioned above, Macica et al. found that 19-HETE made up a significant portion of the arachidonic acid metabolite pool in rabbit intestine and found that 19(R) HETE induced mesenteric dilation (Macica et al., 1993). The fact that the metabolites CYP2P3 forms from arachidonic acid are vasoactive in some systems suggests that CYP2P3 might have a role in vasoactive functions in fish intestine.

Heretofore, there has been little information on metabolism of arachidonic acid by P450 enzymes in fish. A few studies have examined hepatic microsomal metabolism of arachidonic acid in fish. Scup liver microsomes were shown to produce a full spectrum of P450 metabolites with EETs formed in highest quantities followed by HETEs and $\omega-1 / \omega$ hydroxylated metabolites (19- and 20-HETE), respectively. Treatment of fish with $\beta$ naphthoflavone stimulated the formation of EETs and HETEs and had a lesser effect on formation of 19- and 20-HETE. In vitro incubation with the CYP1A inhibitor $\alpha$ naphthoflavone reduced EET and 19- and 20-HETE formation in scup. In this same study, skate liver microsomes produced only EETs and 19- and 20-HETE, $\beta$-naphthoflavone treatment only slightly increased the rate of 19- and 20-HETE formation, and $\alpha-$ naphthoflavone in vitro decreased 19- and 20-HETE formation in $\beta$-naphthoflavone treated skate (Zacharewski et al., 1996). Recent studies have further characterized the arachidonic acid metabolites formed by scup liver microsomes from both untreated and benzo[a]pyrene treated fish. Both control and benzo[a]pyrene treated scup liver microsomes preferentially formed 14,15-EET followed by 11,12-EET and 8,9-EET with little stereoselectivities. Additionally, endogenous EET and DHET levels were quantified in the liver, heart, and kidney of these fish (Schlezinger et al., in prep).

The studies presented here and the studies with the expressed CYP2N proteins identify fish CYP isoforms that metabolize arachidonic acid with distinct regio- and 
stereoselectivities. The occurance of multiple catalysts for arachdionic acid epoxidation and hydroxylation in fish indicate that product, tissue, and cell specificity likely is required for close control of arachidonic acid metabolism; this probable specificity in early diverging vertebrates is like that in mammals (Smith, 1989). The endogenous arachidonic acid metabolites quantified in killifish and other fish suggest that arachidonic acid epoxidation may be an important function for these CYP in fish while the conservation of the 19-HETE metabolite formed by CYP2P3, CYP2J3, and CYP2J4 suggests a conserved function for this metabolite in vertebrates.

Knowledge of the evolutionary origins of a particular CYP addresses the endogenous substrate and function of that CYP. A CYP that has an endogenous function in an early diverging vertebrate such as a fish probably has a mammalian orthologue with a similar endogenous function. This might describe the relationship between the fish CYP2Ps and the mammalian CYP2Js. Members of the CYP2Ps and the CYP2Js potentially are orthologous. The similarity of sequence and tissue distribution might be expected (or insignificant) in a family as large and diverse as the CYP2 family, but their strikingly similar, metabolic profiles for arachidonic acid strongly suggest that these CYP subfamilies and possibly their functions have been conserved through vertebrate evolution. When first identified, the sequence similarity between the CYP2Ps and CYP2Js suggested there may be functional similarities too. As seen here, not only sequence and tissue distribution are similar for the two subfamilies, but also the specificities for metabolism of arachdionic acid are quite similar between CYP2P3 and the CYP2Js. These results highlight the importance of functional properties in establishing homology between CYP forms in different species. The striking similarities between CYP2P3 and CYP2J proteins, especially in the metabolites formed from arachidonic acid, suggest that CYP2P and CYP2J proteins may have arisen from a common ancestral arachidonic acid catalyst. 
CHAPTER 5

SUMMARY AND CONCLUSIONS 


\section{SUMMARY AND CONCLUSIONS}

\section{Cloning and Tissue Distributions}

These studies were initiated in order to examine the diversity, structure, function, and regulation of cytochromes $\mathrm{P} 450$ in early diverging vertebrates. In particular, genes in the CYP2 family were sought in the killifish (Fundulus heteroclitus). Multiple cDNAs from three CYP2 subfamilies were isolated from killifish cDNA libraries. Sequence analyses showed that one cloned from liver is related to CYP2K 1 previously identified in trout (Buhler et al., 1994). The other cDNAs were classified into two new subfamilies, CYP2N and CYP2P. CYP2N1, CYP2P2, and CYP2P3 were cloned from liver, and CYP2N2 was cloned from heart. Northern analyses showed that CYP2P3 gene transcripts are expressed strongly in gut and liver. Likewise, CYP2N1 gene transcripts are expressed strongly in liver and gut but also are expressed in heart and brain. Strikingly, CYP2N2 gene transcripts are expressed most strongly in heart and brain and also are expressed in liver, gut, eye, gill, and kidney. Although many CYP2 genes are involved in the metabolism of exogenous compounds, expression in extrahepatic tissues, especially heart and brain, suggests that the genes identified here might be involved in the metabolism of endogenous substrates as well. As detailed below, the endogenous substrate we first chose to examine was arachidonic acid.

In addition to the cDNA sequences that were cloned, CYP2P1 was cloned from a genomic DNA library. Sequence analysis of CYP2P1 shows that the intron/exon structure for this gene is very similar to the intron/exon structure of mammalian CYP2 genes (Suwa et al., 1985). Interestingly, although fish are not known to respond to the prototypical mammalian CYP2B inducers such as phenobarbital, the 5'-promoter region of the CYP2P1 gene had multiple (5) Barbie boxes or barbiturate responsive elements. Regulatory studies that might be done using the 5'-promoter region of CYP2P1 are discussed below. 
In total, six CYP2 genes in three different subfamilies were isolated from killifish. Previous work has identified other CYP2 subfamilies in teleosts (Yang et al., 1995; Yang et al., 1996). Also genes in families 1, 3, 4, 11, 17, 19, 26, and 52 have been identified in teleosts and elasmobranchs (Stegeman, 1992; Celander et al., 1996)(Stegeman, 1992; Celander et al., 1996; Stegeman, 1992; Celander et al., 1996; Stegeman, 1993; Stegeman and Hahn, 1994; Falckh et al., 1997). Together, these results indicate that fish could have a diversity of CYP forms, more than was originally thought (Stegeman, 1993), and possibly rivalling that in mammals (Table 1). Table 1 is drawn from the Nelson lab homepage (http://drnelson.utmem.edu/nelsonhomepage.html).

Table 1. Families and Subfamilies of Cytochromes P450 Sequenced from Fish

Subfamily

$1 \mathrm{~A}$

2B

$2 \mathrm{~K}$

$2 \mathrm{M}$

$2 \mathrm{~N}$

$2 \mathrm{P}$

$3 \mathrm{~A}$

$4 \mathrm{~T}$

$11 \mathrm{~A}$

17

19

26

$52 \mathrm{~A}$
Species

rainbow trout, scup, plaice, oyster toadfish, killifish four-eye butterfly fish, european seabass, Atlantic tomcod, wild red sea bream

scup (amino-terminus protein sequence)

rainbow trout, killifish

rainbow trout

killifish

killifish

rainbow trout, killifish

rainbow trout

rainbow trout, southern stingray,

rainbow trout, medaka, dogfish

channel catfish, rainbow trout, goldfish, medaka, tilapia

zebra fish

rainbow trout 


\section{Phylogenetic Analyses}

As is implicit in its name, phylogenetic analyses of the killifish CYP2 genes show that killifish CYP2K2 is related most closely to trout CYP2K1. These CYP are 66\% identical at the amino acid level. Phylogenetic analyses also show that the CYP2N and CYP2P genes are related most closely to mammalian CYP2J genes. This relationship holds when the entire gene sequences are compared as well as when only the putative substrate recognition sites (Gotoh, 1992) are analyzed, suggesting that the CYP2Ns, the CYP2Ps and the CYP2Js might have similar catalytic properties. The tissue specific distributions of the CYP2N and CYP2P transcripts also are similar to that of genes in the CYP2J subfamily (Kikuta et al., 1991; Zeldin et al., in press). The fact that these fish CYP are related to the mammalian CYP2Js suggests that the CYP2Ns and CYP2Ps are not unique to fish and thus might have some function in vertebrates generally. Expression of these killifish genes in extrahepatic tissues such as heart and brain suggests that this function might not be solely detoxification, but might involve an endogenous substrate. Because many CYP2J family members metabolize arachidonic acid, this endogenous substrate might be arachidonic acid (Wu et al., 1996; Wu et al., 1997; Zhang et al., 1997).

The studies here support the hypothesis that the CYP2Ns and CYP2Ps are related functionally to the CYP2Js as predicted based on their sequences and tissue distributions. This functional similarity is particularly noteworthy between CYP2P3 and CYP2J2, which have very similar regio- and stereoselective metabolism of arachidonic acid. These functional similarities suggest that despite the probable convergent evolution and occurance of paralogous genes in the CYP2 family, phylogenetic analyses of CYP genes in different taxa can suggest functional relationships.

\section{Functional Studies}

The CYP2Ns and CYP2Ps share both tissue distribution and phylogenetic 
similarities with the mammalian CYP2Js. These similarities suggested possible functional similarities. In order to further examine the relationships between the killifish CYP2Ns and CYP2Ps inferred from transcript expression and sequence analyses, we examined the functions of some of these killifish CYP2 genes. CYP2N1, CYP2N2, and CYP2P3 were co-expressed with cytochrome $\mathrm{P} 450$ oxidoreductase in $S f 9$ insect cells using a baculovirus system, and their metabolism of arachidonic acid, a known CYP2J substrate, was examined.

Arachidonic acid is a 20 carbon, essential fatty acid with four double bonds (C20:4 $\omega-6)$; its metabolism has been studied extensively because of its importance as a precursor to signaling molecules in mammals (Oliw, 1994). Arachidonic acid metabolites are known as eicosanoids and have been studied in a wide variety of organisms. They have been shown to modulate epithelial salt and water transport in mammals (Escalante et al., 1991), frogs, toads (Stanley-Samuelson, 1994), fishes (Wales, 1988), molluscs (Freas and Grollman, 1980) and insects (Petzel and Stanley-Samuelson, 1992). They also have been shown to act in various aspects of reproductive biology in sea stars (Meijer et al., 1986), sea urchins (Schuel et al., 1985), molluscs (Matsutani and Nomura, 1987), insects (Destephano and Brady, 1977), fishes (Wade and Van Der Kraak, 1993), reptiles, and mammals (Liggins et al., 1973). Arachidonic acid metabolites thus are involved in the regulation of cellular processes that are fundamental to organisms in general, and their synthesis is of primary interest.

In most vertebrates, arachidonic acid is derived from the diet. Terrestrial-based food chains tend to have higher levels of arachidonic acid than aquatic-based food chains because high quantities of the arachidonic acid precursor, linoleic acid, are formed in the seeds of seed-bearing plants. Linoleic acid (18:2 $\omega-6)$ is an essential fatty acid in mammals and is elongated and desaturated to form arachidonic acid (Simopoulos, 1991). Arachidonic acid is incorporated most often in the Sn-2 position of intracellular 
phospholipids (Smith, 1989). Before it can be metabolized, arachidonic acid must be released from the membrane by the actions of phospholipase $\mathrm{C}$, phospholipase $\mathrm{D}$, or phospholipases A2. A variety of signals including bradykinin, histamine, thrombin, leukotrienes, and cytokines (Williams and DuBois, 1996) cause the release of arachidonic acid from the membrane. Three enzyme systems metabolize arachidonic acid released from the membranes into eicosanoids (Smith, 1989). These include the cyclooxygenases (cox-1 and cox-2), the lipoxygenases (5-, 12-, and 15-lipoxygenase), and a variety of cytochromes P450, especially those in families 2 and 4 (Figures 1 and 2).

The cyclooxygenases are membrane bound dioxygenases that oxygenate free arachidonic acid to prostaglandin endoperoxide $\mathrm{H}_{2}\left(\mathrm{PGH}_{2}\right)$. These enzymes first form prostaglandin endoperoxide $\mathrm{G}_{2}\left(\mathrm{PGG}_{2}\right)$, and their subsequent peroxidase activity forms $\mathrm{PGH}_{2}$ (Smith and Dewitt, 1996). Prostanoids (prostaglandins and thromboxanes) are synthesized in a cell-specific manner from $\mathrm{PGH}_{2}$. Little is known about the enzymes that catalyze the metabolism of $\mathrm{PGH}_{2}$ although both $\mathrm{PGI}_{2}$ synthase (Graf et al., 1983) and TxA2 synthase (Haurand and Ullrich, 1985) are related to P450 proteins. Prostanoids have effects on ion regulation and water resorption, reproduction, and the vasculature (Stanley-Samuelson, 1994).

In contrast to the cyclooxygenases and cytochromes P450, lipoxygenases are cytosolic proteins. Lipoxygenases are widely distributed in the plant and animal kingdoms (Funk, 1993). In mammals, three different lipoxygenases catalyze the insertion of molecular oxygen into arachidonic acid at positions 5,12 , or 15 to form 5-, 12-, or 15hydroperoxyeicosatetraenoic acid which can be reduced to the corresponding hydroxyeicosatetraenoic acids, lipoxins and leukotrienes: The 12-lipoxygenase is present in platelets and the 15-lipoxygenase is found in leukocytes. The 5-lipoxygenase is involved in leukotriene formation. Leukotrienes have species specific effects on the cardiovascular system (Ford-Hutchinson et al., 1994). 


\section{Diet}
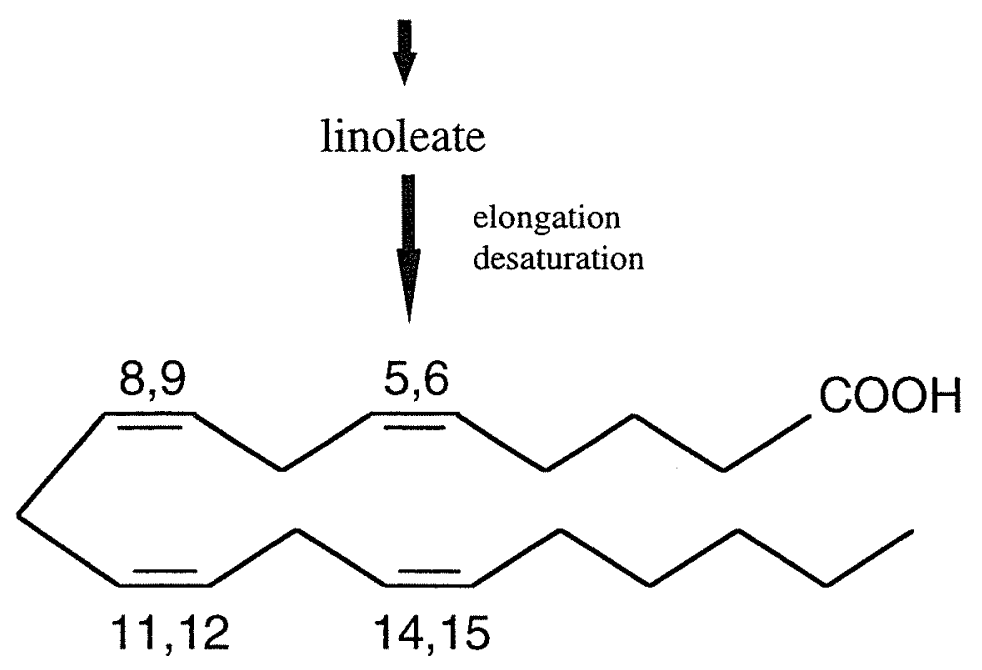

arachidonic acid

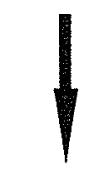

membrane phospholipid

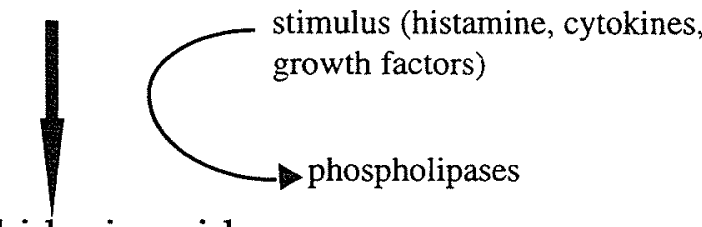

arachidonic acid

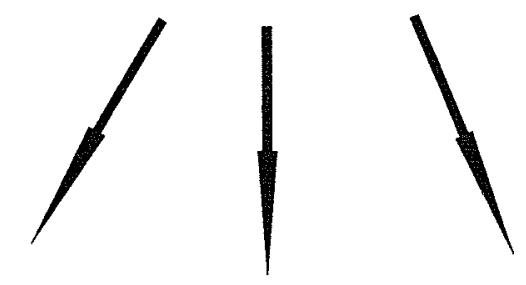

lipoxygenases

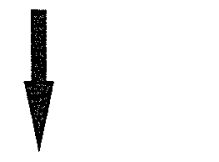

leukotrienes

lipoxins

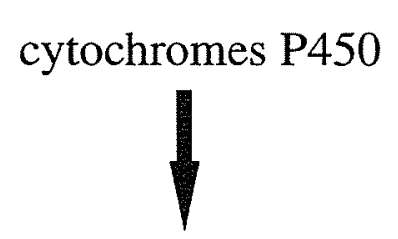

cyclooxygenases

EETs

prostaglandins

HETES

thromboxanes

Figure 1. Arachidonic acid metabolism in vertebrates 


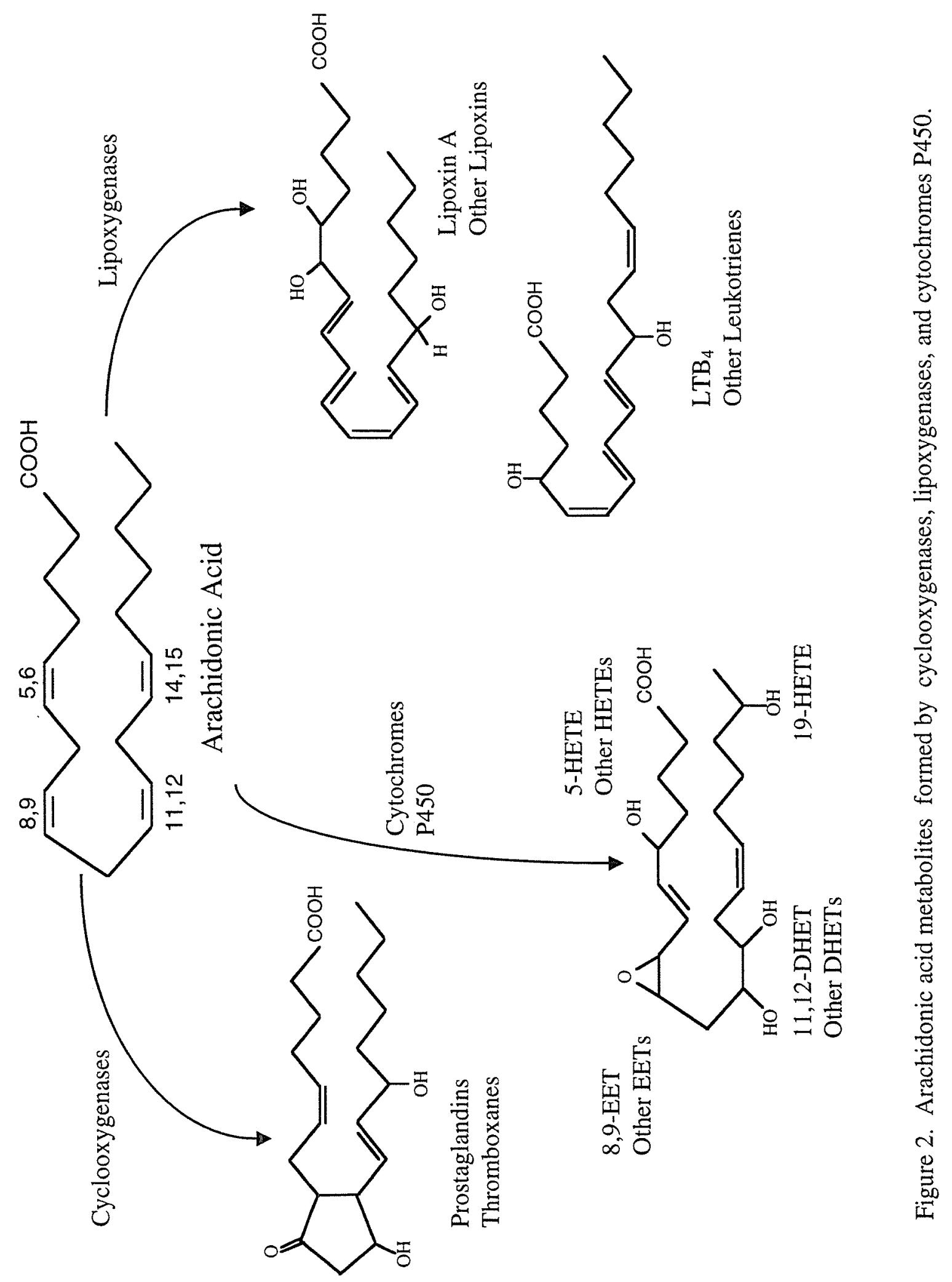


Cytochrome P450 proteins can oxygenate arachidonic acid via olefin epoxidation, oxidation of the $\omega$-side chain, oxidation of allylic or bis-allylic carbons, and oxidation with double bond migration (Oliw, 1994). Although cytochromes P450 originally were thought to metabolize arachidonic acid with little regio- and stereoselectivities, studies with purified and expressed P450s are showing that some P450s do metabolize arachidonic acid with high regio- and stereoselectivities. Cytochrome P450 epoxygenases belong predominantly to the CYP2 family while $\omega$-hydroxylation is catalyzed predominantly by cytochromes P450 in the CYP4 family (Capdevila et al., 1996). Cytochrome P450 epoxygenase reactions have been studied most intensely because the EETs have been shown to be endogenous constituents of numerous tissues and have been shown to have potent biological activities (Capdevila et al., 1984)(Capdevila et al., 1984; Fitzpatrick and Murphy, 1989).

In mammals, the biological effects of arachidonic acid metabolites have been studied in liver, renal cortex and outer medulla, the cardiovascular system, the nervous system, genital glands and exocrine organs, skin tissue, and platelets (McGiff, 1991). Biological activities of arachidonic acid metabolites include change in intracellular calcium concentrations in the liver and kidney, vasoactivity in the kidney and vasculature, release of hormones in the nervous system, and anti-aggregatory effects on platelets (McGiff, 1991).

To date, arachidonic acid studies in fish have concentrated on the cyclooxygenase and lipoxygenase derived eicosanoids. Studies have shown that fish produce both cyclooxygenase and lipoxygenase products which seem to be involved in immune and inflammatory responses in fish (Rowley et al., 1995). In addition, effects of prostaglandins in fish reproduction have been established (Goetz, 1990). Finally, the mitogenic effects of polyunsaturated fatty acids including arachidonic acid have been examined (Gong et al., 1997). These studies showed that arachidonic acid, a prostacyclin analogue, a thromboxane $A_{2}$ analogue, and prostaglandin $F_{2} \alpha$ were mitogenic in coronary, 
vascular smooth muscle.

Only a few studies have examined P450 metabolism of arachidonic acid in fish. Scup liver microsomes were shown to produce a full spectrum of P450 metabolites with EETs formed in highest quantities followed by HETEs and $\omega / \omega-1$ hydroxylated metabolites (19- and 20-HETE), respectively while skate liver microsomes produced only EETs and 19- and 20-HETE (Zacharewski et al., 1996). Recent studies have further characterized the arachidonic acid metabolites formed by scup liver microsomes from both untreated and benzo[a]pyrene-treated fish. Both control and benzo[a]pyrene-treated scup liver microsomes preferentially formed 14,15-EET followed by 11,12-EET and 8,9-EET with little stereoselectivities. Additionally, endogenous EET and DHET levels were quantified in the liver, heart, and kidney of these fish (Schlezinger et al., in prep).

In our analyses of the abilities of expressed CYP2N1, CYP2N2, and CYP2P3 to metabolize arachidonic acid, we found that these enzymes all metabolized arachidonic acid with high regio- and stereospecificities. While CYP2N1 metabolized arachidonic acid more than four times faster than CYP2N2 (435 and $95 \mathrm{pmol} / \mathrm{nmol}$ P450/min for CYP2N1 and CYP2N2, respectively), the CYP2Ns display similar arachidonic acid metabolic profiles. Both CYP2N1 and CYP2N2 catalyzed the NADPH-dependent, regio- and enantioselective epoxidation of arachidonic acid to $11(\mathrm{R}), 12(\mathrm{~S})$-epoxyeicosatrienoic acid with optical purities of $92 \%$ and $70 \%$ for CYP2N1 and CYP2N2, respectively and to $8(\mathrm{R}), 9(\mathrm{~S})$ epoxyeicosatrienoic acid with optical purities of $91 \%$ and $90 \%$ for CYP2N1 and CYP2N2, respectively. CYP2N1 also produced racemic 14,15-EET while CYP2N2 produced $14(\mathrm{~S}), 15(\mathrm{R})-\mathrm{EET}$ with an optical purity of $68 \%$. In contrast, the CYP2Ns formed different HETE products from arachidonic acid: CYP2N1 hydroxylated arachidonic acid to a variety of mid-chain, cis-trans-conjugated dienols (5-, 16-, 12-, 9-, and 11-hydroxyeicosatrienoic acid in order of decreasing amounts) while CYP2N2 mainly produced 9-, an unidentified-, and 20- hydroxyeicosatrienoic acid (also in order of decreasing amounts). 
The arachidonic acid metabolic profile of CYP2P3 was very different from those of the CYP2Ns. CYP2P3 metabolized arachidonic acid (catalytic turnover of $135 \mathrm{pmol} / \mathrm{nmol}$ of cytochrome $\mathrm{P} 450 / \mathrm{min}$ ) to 14,15 -epoxyeicosatrienoic acid, 11,12-epoxyeicosatrienoic acid, and 8,9-epoxyeicosatrienoic acid as the principle reaction products (these EETs comprised $83 \%$ of the total products) and to 19-hydroxyeicosatrienoic acid as the only nonepoxide product. CYP2P3 epoxidation of arachidonic acid was highly enantioselective for 14(R), 15(S)-epoxyeicosatrienoic acid (72\% optical purity) and for $8(\mathrm{~S}), 9(\mathrm{R})$ epoxyeicosatrienoic acid (76\% optical purity), and CYP2P3 hydroxylation of arachidonic acid was highly enantioselective for 19(R)-hydroxyeicosatrienoic acid (78\% optical purity).

Killifish liver microsomes also were shown to metabolize arachidonic acid primarily to epoxyeicosatrienoic acids and their hydration products, dihydroxyeicosatrienoic acids. Most of the products of microsomal metabolism could result from the metabolic activities of CYP2N1, CYP2N2, and CYP2P3. In fact, the liver microsomes formed the different EET regioisomers in similar ratios to those formed by CYP2N1 and CYP2N2, and one liver HETE product (16-HETE) also is formed by CYP2N1 while the other (19-HETE) also is formed by CYP2P3. Finally, both liver and gut tissues were shown to have endogenous EET and DHET metabolites. Amounts of different EET regioisomers ranged from 44 to $113 \mathrm{ng} / \mathrm{g}$ tissue and those of the DHET regioisomers ranged from 16 to $44 \mathrm{ng} / \mathrm{g}$ tissue. Since all of these fish had been feeding on the same diet for more than a year, the variability between fish in the levels of EETs and DHETs may reflect some type of regulation of arachidonic acid and/or its metabolites.

These results with expressed CYP2N1, CYP2N2, and CYP2P3 proteins demonstrate a catalytic diversity of arachidonic acid metabolites in early vertebrates and indicate that the novel CYP2N and CYP2P genes encode arachidonic acid epoxygenases and hydroxylases. The regio- and stereoselectivities of the metabolites formed by CYP2P3 are strikingly similar to those formed by the mammalian CYP2Js suggesting that CYP2P 
proteins may represent early vertebrate forms of the arachidonic acid catalysts that gave rise to the mammalian CYP2Js. Importantly, functional studies with CYP2N1 and CYP2N2 may elucidate the fundamental roles of their activities in vertebrates, particularly in organs such as heart and brain.

\section{FUTURE DIRECTIONS}

\section{Biological Effects}

CYP2N1, CYP2N2, and CYP2P3 all metabolize arachidonic acid with high regioand stereo-selectivities. Whether the metabolites formed have physiological roles in fish is unknown. Based on mammalian studies, possible roles for arachidonic acid metabolites include change in intracellular calcium concentrations in a variety of tissues, vasoactivity in the vasculature, release of hormones in the nervous system, and anti-aggregatory effects on platelets (McGiff, 1991). Possible ion flux effects are being pursued using primary cell cultures and a vibrating ion probe that can measure ion flux across a cell membrane (Jaffe and Nuccitelli, 1974). These studies were begun with a fish hepatoma cell line from Poeciliopsis lucida, PLHC-1 (Hightower and Renfro, 1988). Unlike non-cancerous cells that had been examined with this probe, these cells showed minimal, homeostatic calcium flux. In order to be more physiologically relevant, we switched to primary hepatocyte cultures from killifish liver tissues (see Appendix B). These cells do show a calcium flux at the cell membrane. Currently, the effects of the arachidonic acid epoxides and 19-HETE on calcium flux are being examined. Because many arachidonic acid metabolites have been shown to activate sodium/potassium ATPases, potassium flux also might be interesting to examine with this probe. Given the potent biological effects seen with mammals, likely these arachidonic acid metabolites will have effects in fish. 
$\underline{\text { Regulatory Studies }}$

As well as direct approaches to measure biological effects, knowledge of the regulation of both the transcripts and the proteins might suggest possible physiological functions for these CYP. Levels of both transcript and protein need to be examined in killifish using Northern and Western analyses. Changes in levels and/or tissue distributions throughout development might suggest function. In addition, responses to a variety of substrates might suggest function. Interesting substrates might include 1) peroxisome proliferators because of their effects on the release of arachidonic acid and their known regulatory effects on the CYP4As which metabolize arachidonic acid (Simpson, 1997); moreover, because an arachidonic acid analogue (Keller et al., 1993) and arachidonic acid metabolites (Krey et al., 1997) were found to be a potent ligands for the peroxisome proliferator activated receptor; 2) aryl hydrocarbon receptor (AhR) ligands because an arachidonic acid metabolite is postulated to be an endogenous AhR ligand (Nebert, 1994); 3 \& 4) oxygen and mechanical stretch because of their potential importance in regulating heart function; 5) TPA because of its effect on the phospholipase C pathway (Fischer and Slaga, 1985); 6) phenobarbital because of the multiple Barbie boxes in the $5^{\prime}$-untranslated end of the CYP2P1 gene; 7) fatty acids because often cytochromes $\mathrm{P} 450$ are regulated by the same substrates they metabolize and because fatty acids bind to the BM3RI repressor (Wolf et al., 1996) which interacts with Barbie boxes. These dose response experiments would be much easier in a cell line or primary tissue culture such as used for the ion flux studies.

Before protein levels can be examined, antibodies need to be made against these proteins. Ideally, these antibodies would be isoform specific. Such specificity is unlikely given the amino acid similarities between the CYP2N and CYP2P isoforms. Antibodies made against the entire protein probably would cross-react with each other even if preabsorbed against each other. (Anti-CYP2J2 was preabsorbed against SF9 lysate and 
still cross-reacts with a wide spectrum of insect proteins). Peptide antibodies might be more specific than antibodies made against the whole protein. In fact, Zhang et al. have made anti-CYP2J4 antibodies against a synthetic peptide corresponding to amino acid residues 427-441 (Zhang et al., 1997) (anti-CYP2J4 cross-reacts with at least three different size bands in Western analyses suggesting that it probably is not isoform specific but may be subfamily specific), and Edwards et al. have had success making subfamily specific peptide antibodies against the C-terminus of a variety of CYP proteins (Edwards et al., 1995).

For purification purposes, CYP2N1, CYP2N2, and CYP2P3 were expressed without reductase in $S f 9$ cells using a different baculovirus expression system (Invitrogen). This system was used because it gives higher expression levels than the Pharmingen system (Darryl Zeldin, personal communication). We were able to express all three CYP successfully using this system, but we have been unsuccessful in our attempts to purify CYP protein for antibody production. We have used DEAE cellulose, octylaminosepharose, hydroxylhapatite, and chloroamphetamine-linked cellulose columns to no avail. We have gotten enrichment rather than purification (see Appendix C).

In addition to knowledge of the regulation and timing of expression of these genes and proteins, knowledge of the cell types that express these CYP might suggest possible physiological functions. Transcript expression could be localized using in situ hybridization techniques, and protein expression could be localized using immunohistochemical techniques. Unsuccessful attempts were made using in situ hybridization techniques, and these should be explored further (see Appendix D).

As well as looking at the regulation of transcripts and proteins, regulation also might be examined with the 5'-promoter regions of these genes. CYP2P1 is the obvious gene to start with because the 5 '-end is cloned already. Alternatively, the killifish genomic library could be screened with the cDNA probes to isolate other CYP2N and CYP2P 
genomic clones. The 5'-ends could be targeted using only the 5'-ends of the cDNAs to probe this library, or oligonucleotide probes could be specifically designed to the 5 '-ends. Reporter constructs using the 5'-promoter regions of these genes could be used to look for positive and negative regulatory elements. These constructs would include a suite of nested deletions attached to a reporter gene which could be transfected into a fish hepatocyte cell line. Dose-response experiments also might be done with these constructs. In addition, foot-print analyses might be done with the 5'-promoter region to locate DNA binding sites. Again, these might be done with treated fish. Finally, if a responsive element is prominent in the sequence, such as the Barbie box found in the CYP2P1 gene, this responsive element might be used in gel-shift assays to see if it really is a responsive element. It might be interesting to use the CYP2P1 Barbie box sequence with control and phenobarbitaltreated rat nuclear extracts to see if this sequence binds any proteins. Additionally, footprint analyses might be done with these extracts.

\section{Other $\underline{\text { Substrates }}$}

Finally, given the broad substrate specificities of many CYP in the CYP2 family, other substrates need to be used in metabolism studies. Choice of substrate would be highly dependent on the dose response results, but substrates that may be significant in fish are the $\omega-3$ fatty acids, eicosapentaenoic acid (EPA) and docosahexaenoic acid (DHA). In most mammalian tissues, the ratio of arachidonic acid: $\omega-3$ fatty acids is $2: 1-6: 1$ in structural lipids. In fish, this ratio is reversed because most aquatic food chains are based on nonseed bearing plants (i.e. phytoplankton) that produce little arachidonic acid (Weber, 1990). Interestingly, in all mammalian species examined, the arachidonic acid: $\omega-3$ fatty acid ratio is maintained at $1: 1$ in certain tissues even when diets are depleted of $\omega-3$ fatty acids. These tissues include the brain, testes and retina (Crawford and Harbige, 1988; Simopoulos, 1991). The maintenance of $\omega-3$ fatty acids in these specific tissues suggests 
that they might have important functions in these tissues. The high levels of $\omega-3$ fatty acids in many fish species make $\omega-3$ fatty acids interesting putative substrates for the CYP2Ns and CYP2Ps. Often, P450s that metabolize arachidonic acid metabolize EPA and DHA with similar regioselectivities (VanRollins, 1990). In mammals, the metabolites derived from EPA and DHA are generally less potent biologically than those derived from arachidonic acid. Although this has been seen in fish as well (Bell et al., 1995), some metabolites derived from $\omega-3$ polyunsaturated fatty acids such as the 5-series lipoxins are as potent as the corresponding arachidonic acid metabolite i.e. the 4-series lipoxins (Sharp et al., 1992). In mammals as well, lipoxin A5 and lipoxin A4 have similar potencies for inducing superoxide anion generation and potentiating contraction of isolated rat tail arteries (Lam and Wong, 1988). The biological effects of any EPA and DHA metabolites would be interesting to examine in fish, especially marine fish.

The intriguing question that remains is what are the functions of the CYP2Ns and CYP2Ps in extrahepatic tissues, especially in heart and brain? The phylogeny of the genes, the tissue distribution of the transcripts, and the characterization of the metabolites formed from arachidonic acid begin to address this question. Further studies on the regulation and expression of these CYP, on the biological activities of the arachidonic acid metabolites formed by these CYP, as well as on other substrates for these CYP may answer this question. 


\section{APPENDICES}


APPENDIX A: NATURAL PRODUCTS $\underline{\text { AND }}$ P450s

Natural products increasingly are being recognized as substrates and/or inducers of cytochromes P450. The diversity of mammalian cytochromes $\mathrm{P} 450$ in gene families 1-4, especially family 2 , has been suggested to have been driven by the consumption of terrestrial plants with their evolving chemical defenses (Nelson and Strobel, 1987). The role of natural products in the environment is important to questions concerning the evolution of cytochrome $\mathrm{P} 450$ proteins and regulatory systems. Their role also is crucial to interpretations of anthropogenic versus natural product induction of cytochrome P450 proteins for use as pollutant biomarkers. Chemical-biological interactions are a fundamental basis of many ecosystems. As prominent enzymes in the metabolism of xenobiotics, cytochromes $\mathrm{P} 450$ probably have major roles in modulating these interactions.

Chemical-biological interactions involving fish and natural products could be important for the ecology, toxicology, and evolution of these animals. The suspected role of natural products in modulating the expression of xenobiotic-metabolizing cytochromes P450 is potentially fundamental to these interactions but is not known in any detail. Our overall goal is to establish the regulation of selected cytochrome P450 genes that may be linked to chemical-biological interactions between natural products and aquatic vertebrates.

Indications that regulation of cytochrome $\mathrm{P} 450$ gene expression in fish liver may be linked to natural products in the diet have come from studies of reef fishes. Comparative studies showed elevated cytochrome P450 protein levels in butterfly fish that feed on gorgonians which contain prostaglandins and terpenoids versus congeneric species that do not feed on gorgonians (Vrolijk and Targett, 1991; Vrolijk et al., 1994). In addition, Bermuda chub from an unpolluted site in Bermuda had total hepatic microsomal cytochrome P450 levels far in excess (5 to 10-fold) of those typically seen in most temperate and freshwater species (Stegeman et al., 1990), 2 to 4 times the levels seen in the 
butterfly fish, and 10 to 20 times the total cytochrome P450 seen in untreated scup. Bermuda chub are one of the few fish species that consume copious amounts of plant material (Horn, 1989).

We were interested in establishing the profiles of P450 expression in fish species with prominent marine or terrestrial plant components in their diets. We concentrated on the marine herbivorous fish, Bermuda chub, which previously was shown to have strikingly high levels of liver microsomal cytochrome P450 (Stegeman et al., 1990). These fish had insignificant levels of CYP1A that would not account for the bulk of cytochrome P450 present in the liver but did have abundant CYP2 cross-reacting protein. Bermuda chub are plant feeding fishes, primarily consuming algae (Nelson, 1984), and several species of Kyphosus prefer brown algaes (Horn, 1989). These fish consume fucoid algaes which are rich in phlorotannins, tannins constructed of phloroglucinol moieties linked by aryl-aryl or aryl-ether bonds or of a mixed type (Faulkner and Fenical, 1977). Thus, these fish likely encounter multiple dietary natural products.

\section{MATERIALS AND METHODS}

Fish: 1995 Bermuda chub from Bermuda were trapped in fish pots and held in a laboratory for 2-24 hours before use. Fish were either sacrificed immediately or injected with phenobarbital (70 ug/kg in saline/15\%DMSO). Four fish were injected with phenobarbital and four fish were injected with carrier (saline/15\%DMSO) and sacrificed 2 days later. Only two of the injected fish lived. The two that lived had been injected with phenobarbital. All the others died probably due to gut rupture during injection. Microsomes were prepared immediately from liver tissues as previously described (Stegeman et al., 1990). RNA also was prepared immediately using RNA STAT 60 (Tel Test 'B'). 1994 Bermuda chub from Bermuda and the Bahamas were speared and their 
livers were frozen immediately. Microsomes were prepared from these frozen livers as above. Microsomes previously prepared from the 1988 Bermuda chub were used for comparisons.

Total P450 Content: Cytochrome P450 content was determined using a modification of Omura and Sato (Omura and Sato, 1964). The difference between the absorbance peak at $450 \mathrm{~nm}$ and the baseline absorbance at $490 \mathrm{~nm}$ for carbon monoxidebound, non-reduced versus reduced microsomes was determined spectrophotometrically (Schimadzu). Protein was determined using the bicinchoninic method of Smith et al. (Smith et al., 1985) and total P450 content was normalized to protein content in order to compare fish.

Specific P450 Content: Western analyses, using a suite of different cytochrome P450 antibodies, were done to identify (at least at the level of family) the cytochrome P450 protein(s) expressed. Antibodies against CYP proteins in families 1-4 were used. These included the monoclonal anti-scup CYP1A1 (Kloepper-Sams et al., 1987; Morrison et al., 1995), anti-scupA (CYP3A-like) (Woodin et al., 1988; Celander et al., 1996), polyclonal anti-scupB (CYP2B-like) (Woodin et al., 1988), and polyclonal anti-rat4A (CYP4A) (Gibson, 1989). Western blot analyses were performed with microsomes prepared from liver tissues as previously described (Stegeman et al., 1990). 100 ug of microsomal protein was run on SDS-PAGE gels and transferred overnight to nylon sheets (Schleicher and Schuel, Keene, $\mathrm{NH})$ at $4^{\circ} \mathrm{C}$ (BioRad transblot). Depending on the primary antibody, the appropriate, alkaline-phosphatase conjugated, secondary antibody was used. The cross-reactive proteins were visualized on the nylon membranes using enhanced chemiluminescence (RAD FREE Kit for Chemiluminescent Detection of Western Blots, Schleicher and Schuel, Keene, NH). Antibody staining was quantified densitometrically. 
Scup liver microsomes were used as controls.

\section{RESULTS}

The cytochrome P450 levels in the Bermuda chub caught in 1994 and 1995 in Bermuda and the Bahamas were low compared to those of the Bermuda chub caught in 1988 (Figure 1). The 1988 Bermuda chub had 1.7-22 times more total cytochrome P450 levels than the chub caught in 1994 and 1995. The 1988 fish had 1.43 and 2.04 nmol total P450/mg protein. In contrast, the chub caught in 1994 and 1995 had cytochrome P450 levels that ranged between a high of $0.82 \mathrm{nmol} \mathrm{P} 450 / \mathrm{mg}$ protein and a low of $0.09 \mathrm{nmol}$ P450/mg protein. Similarly, the levels of cross-reacting proteins in families 2 and 3 were not strikingly high in these fish compared to the 1988 fish (Figure 2). Three of the samples had higher CYP1A-cross-reacting proteins than the 1988 Bermuda chub suggesting that these fish might be from polluted waters (Stegeman and Woodin, 1988; Stegeman et al., 1988). The 1988 Bermuda chub had much higher CYP2 and CYP3A-cross-reacting proteins than the other fish. All of the chub had similar levels of CYP4A-cross-reacting proteins. 


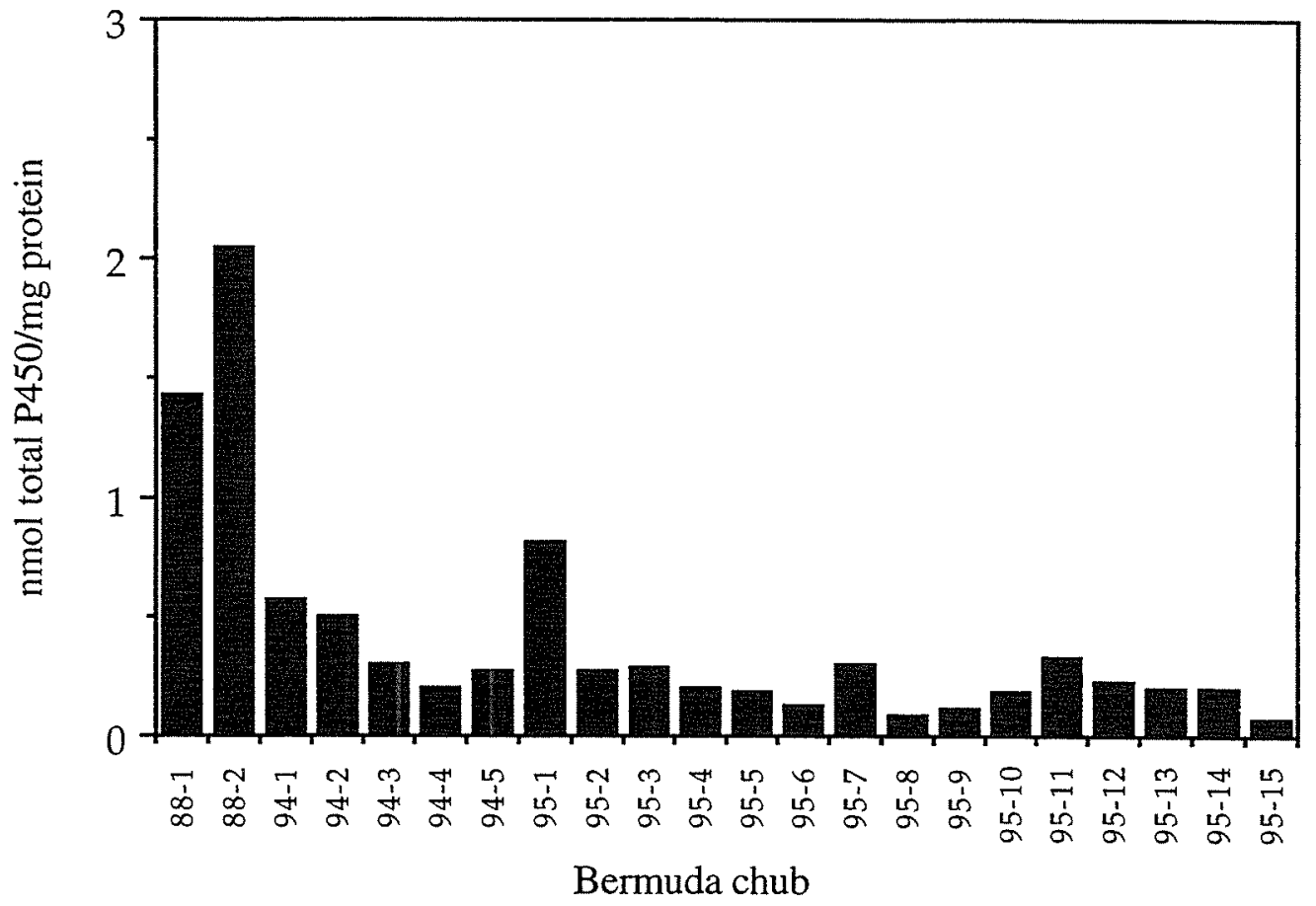

Figure 1. Total cytochrome P450 levels in Bermuda chub caught in 1988 (88-1-2), 1994 (94-1-5), and 1995 (95-1-15). Cytochrome P450 content was determined using a modification of Omura and Sato (Omura and Sato, 1964). Protein was determined using the bicinchoninic method of Smith et al. (Smith et al., 1985), and total P450 content was normalized to protein content in order to compare fish. 
94-1 $94-2 \quad 94-3 \quad 94-4 \quad 94-5 \quad 88-1 \quad 88-2$

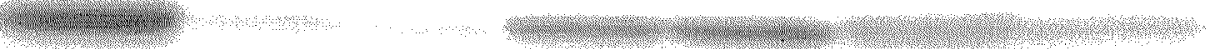

Anti-CYP1A

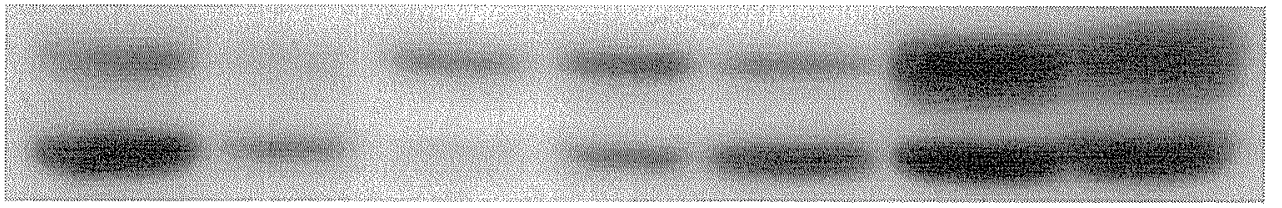

Anti-CYP2B-like

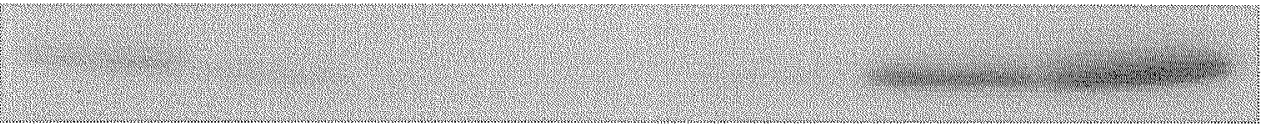

Anti-CYP3A-like

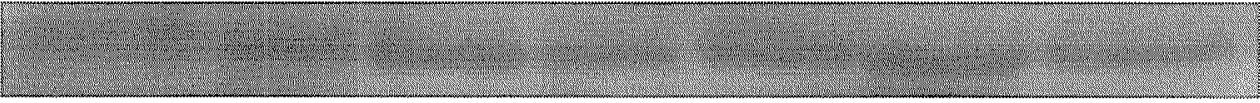

Anti-CYP4A

Figure 2: Immunoblots with Bermuda chub hepatic microsomes. Microsomes were from fish caught in 1994 (94-1-5) and 1988 (88-1-2). $100 \mathrm{ug}$ of microsomal protein was run on SDS-PAGE gels and transferred overnight to nylon sheets (Schleicher and Schuel, Keene, $\mathrm{NH}$ ) at 4 degrees C. Depending on the primary antibody, the appropriate alkaline-phosphatase conjugated secondary antibody was used. The cross-reactive proteins were visualized on the membranes using enhanced chemiluminescence (RAD FREE Kit for Chemiluminescent Detection of Western Blots, Scheicher and Schuel, Keene, NH). 


\section{DISCUSSION}

The 1988 Bermuda chub have much higher levels of cytochrome P450 than any of the other Bermuda chub examined. In fact, the 1994 and 1995 Bermuda chub have total cytochrome P450 levels similar to temperate, carnivorous teleosts (Stegeman et al., 1985). In addition, the 1994 chub with higher total P450 levels had more CYP1A1 cross-reacting protein than the 1988 chub suggesting that their higher total P450 might be due in part to CYP1A1 induction which might result from environmental pollution (Stegeman et al., 1981; Stegeman and Woodin, 1988).

If dietary natural products indeed are affecting the levels of total cytochrome P450 in these fish, different diets of these fish might account for these differences. Gut contents were analyzed only for the 1995 Bermuda chub and showed that these fish were feeding predominantly on a green algae, not the more chemically defended brown algaes (data not shown). Feeding studies would more fully address whether or not dietary natural products were affecting the total cytochrome P450 levels. Alternatively, comparisons between chub feeding on different species of algae including brown algaes also would address this question.

A potentially confounding difference between these groups of fish is the species of chub. The 1988 fish were identified as Kyphosus sectatrix. In contrast, the chub from the Bahamas and from Bermuda in 1994 and 1995 were identified as Kyphosus incisor. These two species of chub are morphologically very similar and will school together. Differences between them lie in the number of gill rakers and the number of dorsal and anal rays. The 1995 Bermuda chub were identified based on the number of dorsal and anal rays. The 1988 Bermuda chub were not identified based on these characteristics. The microsomal protein profiles of the different Bermuda chub are different suggesting that these fish indeed are different species. In fact, in 1995, all of the Bermuda chub found around 
Bermuda were identified as Kyphosus incisor (Dr. Brian Luckhurst, personal communication). Thus, differences in cytochrome P450 levels and profiles might be due to species differences as well as or rather than diet or other factors.

Based on these results, future work should consider similar studies with Bermuda chub known to be feeding on chemically defended brown algaes. Bermuda chub from Australian and New Zealand waters are known to feed on some of the most chemically defended brown algaes known (Horn, 1989). Feeding experiments also might be considered since Bermuda chub will feed on white bread (Dr. Nancy Targett, personal communication) which has no potent natural products. The gut through-put time for Bermuda chub is $\sim 21$ hours (Horn, 1989) so these studies would need to be done with aerated saltwater tanks. This may not be possible on site. Gut content analyses also should be done. 


\section{APPENDIX B: PRIMARY HEPATOCYTES}

Primary hepatocytes made from killifish livers were used for preliminary calcium flux studies. They also were discussed for possible dose response experiments. The following protocol was developed for the calcium flux studies. Both trypsin and collagenase were used to digest the livers, and the trypsin protocol gave better results. These hepatocytes were used $24-48$ hours after being plated. Future studies with these primary cultures should consider events such as dedifferentiation that might occur over time. See Baksi and Frazier for a review on fish hepatocytes (Baksi and Frazier, 1990).

Solutions: (Sterilize all solutions, also $\mathrm{pH} \sim 7.1$ )

$\mathrm{Ca}^{++}$free Ringer's ( $\mathrm{g} / \mathrm{L}$ unless otherwise noted)

$\begin{array}{lc}\mathrm{NaCl} & 10.337 \\ \mathrm{KCl} & 0.115 \\ 7 \mathrm{H} 2 \mathrm{OMgSO} 4 & 1.6 \\ \mathrm{Na}_{2} \mathrm{HPO}_{4} & 0.389 \\ \mathrm{~K}_{3} \mathrm{PO}_{4} & 0.063 \\ \text { glucose } & 0.800 \\ \text { (BSA } & 20.0) \\ \text { EGTA } & 5 \mathrm{mls} \text { of } 100 \mathrm{mM} \\ \text { P/S } & 10 \mathrm{mls} \text { (Sigma P-0781, 10,000U pen., } 10 \mathrm{mg} / \mathrm{ml} \text { strep.) } \\ \text { Fungizone } & 1 \mathrm{ml} \text { of } 10 \mathrm{mg} / \mathrm{ml}\end{array}$

Trypsin Solution (same solution used for cell culture, $g / \mathrm{L}$ unless otherwise noted)

$\begin{array}{ll}\mathrm{NaCl} & 8 \\ \mathrm{KCl} & 0.4 \\ \mathrm{Na}_{2} \mathrm{HPO}_{4} 7 \mathrm{H}_{2} \mathrm{O} & 0.045 \\ \mathrm{KH}_{2} \mathrm{PO}_{4} & 0.03 \\ \text { Phenol Red } & 0.0012 \\ \text { Trypsin } & 0.2 \% \\ \text { EDTA } & 0.05 \mathrm{M}\end{array}$

$\underline{\mathrm{MEM} / 10 \%} \underline{\mathrm{CS}}$ (same media used for PLHC cells with added P/S and fung.)

MEM (Sigma M-0643)

$\begin{array}{ll}\text { Calf Serum } & 100 \mathrm{ml} / \mathrm{L} \\ \text { Hepes free acid } & 5.96 \mathrm{~g} / \mathrm{L} \\ \mathrm{NaHCO} 3 & 2.2 \mathrm{~g} / \mathrm{L} \\ \mathrm{P} / \mathrm{S} & 10 \mathrm{ml} / \mathrm{s} \\ \text { fungizone } & 1 \mathrm{ml} / \mathrm{L}\end{array}$


Kill fish and place livers in $\mathrm{Ca}^{++}$-free Ringer's on ice $(\sim 10 \mathrm{mls} / \mathrm{liver})$. Transfer livers to a sterile hood and place them in fresh buffer in a sterile petri dish. Mince livers with a sterile scalpel. Remove buffer and place livers in trypsin solution $(\sim 10 \mathrm{ml} / \mathrm{liver}$, same one used to trypsinize cells) in sterile $50 \mathrm{ml}$ tubes. Incubate at room temperature for 20 minutes with occasional shaking. Smash liver pieces with homogenizer or sterile spoon against the tube wall. Filter through 4 layers of sterile cheesecloth. Spin $100 \mathrm{xg}, 5 \mathrm{~min}$. Resuspend in buffer or $10 \% \mathrm{CS} / \mathrm{MEM}$ and spin again. Yield is about $1 \mathrm{X} 10^{6}$ cells/g fish. Resuspend in media. Count viable cells (with trypan blue) and plate. The hepatocytes take $\sim 1$ day to sit down and another day to flatten.

Notes: Plate $\sim 500,000$ cells/ml for $\sim 70-80 \%$ confluence. The extracellular calcium is chelated to promote disaggregation. The BSA is to prevent clumping. (BSA makes the media very difficult to sterile filter and is not necessary. The trypsin solution for cellculture does not have BSA, and so BSA is absent at probably the most critical point. CS can substitute for BSA (will also stop trypsin so cannot use during trypsin digestion), and so may want to resuspend and wash cell pellet in MEM $+\mathrm{CS}$ ). The centrifugation speed should be $50 \mathrm{~g}$ but we used $100 \mathrm{~g}$ since speed already calculated. At $50 \mathrm{~g}$ the nonhepatocytes should remain in the supernate. Hepatocytes are the primary cell type, but red blood cells and glial cells also were present in the cultures. Any bacterial contamination disappeared after a few days in culture. These cells were successfully trypsinized and passaged four days later.

These primary hepatocytes were used to examine the effects of arachidonic acid metabolites on calcium flux. Only preliminary studies have been done. Cells were plated at $1 \times 10^{6} / 4 \mathrm{ml}$ dish. The metabolites tested included 14,15-, 11,12-, and 8,9-EET, 5,6- 
EET-Me, 19(R)-HETE, and 19(S)-HETE. These metabolites were used at $1 \mathrm{uM}$ final concentrations. Only the 8,9-EET appeared to have an effect on calcium flux, but this result was uncertain because the probe may not have been equilibrated. The small size of the hepatocytes compared to the probe makes probe manipulations difficult. These studies need to be repeated. 


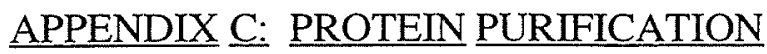

Multiple attempts were made to purify the CYP2N1 expressed protein for antibody production. No column purification scheme has been successful. Thus, antibodies generated against synthetic peptides might be considered. Theoretically, the crossreactivity with peptide antibodies should be much less than with whole proteins: due to the similarities between the CYP2Ns and the CYP2Ps, antiserum produced against purified protein has a great potential for cross-reactivity with the other proteins owing to the multiple epitopes on these similar protein antigens. Described below are the column purification methods we tried. Peptide antibodies also are discussed further.

Based on the similarities between the CYP2Ns and the CYP2Js, we initially tried the same purification scheme that was used to purify CYP2J2 (Wu et al., 1996). Briefly, Sf9 cells expressing recombinant CYP2N1 were harvested 72 hours after infection, washed once with phosphate-buffered saline, and lysed in $0.1 \mathrm{M}$ sodium phosphate $(\mathrm{pH}$ 7.4) containing $20 \%(\mathrm{v} / \mathrm{v})$ glycerol, $1 \%(\mathrm{w} / \mathrm{v})$ sodium cholate, $0.1 \mathrm{uM}$ EDTA, and $0.1 \mathrm{uM}$ dithiothreitol. Insoluble protein was removed by centrifugation for 1 hour at $100,000 \mathrm{xg}$. The P450 content of the crude lysate was determined spectrally and adjusted to $1 \mathrm{uM}$ concentration. The sodium cholate concentration was adjusted to $0.4 \%$ with a dropwise addition. This lysate was loaded onto a $\omega$-aminooctyl-agarose column equilibrated with $0.1 \mathrm{M}$ potassium phosphate ( $\mathrm{pH} 7.4)$ containing $20 \%(\mathrm{v} / \mathrm{v})$ glycerol, 0.1 uM EDTA, 0.1 uM dithiothreitol, and $0.4 \%$ (w/v) sodium cholate (buffer A). The bound CYP2N1 was eluted with buffer A containing 0.4\% Emulgen 911 (Kao Chemical Co., Tokyo). After dialysis against 200 volumes of $10 \mathrm{mM}$ Tris- $\mathrm{Cl}$ buffer ( $\mathrm{pH} 7.4$ ) containing $20 \%(\mathrm{v} / \mathrm{v})$ glycerol, $0.1 \%(\mathrm{w} / \mathrm{v})$ sodium cholate, $0.1 \mathrm{uM}$ EDTA, and $0.1 \mathrm{uM}$ dithiothreitol (buffer B), the CYP2N1 sample was loaded onto a $1 \times 5 \mathrm{~cm}$ hydroxylapatite column equilibrated with buffer B containing $0.4 \%(\mathrm{v} / \mathrm{v})$ Emulgen 911 . The column was washed with 6 column 
volumes of equilibration buffer, 4 column volumes of $0.04 \mathrm{M}$ sodium phosphate ( $\mathrm{pH} 7.4)$ containing $20 \%(\mathrm{v} / \mathrm{v})$ glycerol, $0.1 \mathrm{uM}$ EDTA, $0.1 \mathrm{uM}$ dithiothreitol, $0.1 \%(\mathrm{w} / \mathrm{v})$ sodium cholate, and $0.4 \%(\mathrm{v} / \mathrm{v})$ Emulgen 911. The bound CYP2N1 was eluted with $0.1 \mathrm{M}$ sodium phosphate ( $\mathrm{pH} 7.4)$ containing 20\% (v/v)glycerol, 0.1 uM EDTA, 0.1 uM dithiothreitol, $0.1 \%(\mathrm{w} / \mathrm{v})$ sodium cholate, and $0.4 \%$ Emulgen 911 and dialyzed versus 200 volumes of buffer B. Fractions were run on SDS-Page gels and visualized with comassie blue. Unlike the purification found for CYP2J2, CYP2N1 was not significantly purified with this method. (Western blot analyses of $S f 9$ insect cell microsomes containing the CYP2N and CYP2P3 proteins using anti-CYP2J2 showed that anti-CYP2J2 cross-reacted with most of the microsomal insect proteins suggesting that CYP2J2 may not have been highly purified either).

We also tried the procedure described by Warner (Warner, 1982) using DL-pchloroamphetamine coupled to activated $\mathrm{CH}$ sepharose $4 \mathrm{~B}$ as directed by the manufacturers (Pharmacia). The swollen gel was incubated with 3-fold molar excess of DL-pchloroamphetamine at room temperature for 2 hours in $100 \mathrm{mM}$ sodium bicarbonate buffer (pH 8.0) containing $1 \mathrm{M} \mathrm{NaCl}$. After coupling, the gel was washed with three cycles of $100 \mathrm{mM}$ Tris buffer ( $\mathrm{pH} 8.0$ ) then $100 \mathrm{mM}$ sodium acetate buffer ( $\mathrm{pH} \mathrm{4.5),} \mathrm{both}$ containing $1 \mathrm{M} \mathrm{NaCl}$. The gel was washed with 1 liter of deionized water and equilibrated in $30 \mathrm{mM}$ sodium phosphate buffer ( $\mathrm{pH} \mathrm{7.4)} \mathrm{containing} 20 \%$ glycerol, $0.06 \%$ emulgen 911 , and $0.16 \%$ sodium cholate (starting buffer). The equilibrated gel was packed in a 0.5 $x 10 \mathrm{~cm}$ column in starting buffer. Microsomal fractions were resuspended in $30 \mathrm{mM}$ sodium phosphate buffer ( $\mathrm{pH} 7.4$ ) containing $20 \%$ glycerol, $0.2 \%$ emulgen $911,0.5 \%$ sodium cholate, $0.1 \mathrm{mM}$ EDTA (solubilization buffer). Before loading, this suspension was diluted 3-fold with $30 \mathrm{mM}$ sodium phosphate buffer containing $20 \%$ glycerol and applied to the column at a rate of $0.5 \mathrm{ml} / \mathrm{min}$. Columns were washed with starting buffer containing $100 \mathrm{mM} \mathrm{NaCl}$. The P450 was eluted with solubilization buffer. Again, 
CYP2N1 was not significantly purified using this procedure.

Due to this lack of purification, peptides might be a more profitable approach towards generating antibodies. As mentioned, Edwards et al. have had success with the Ctermini of CYP proteins which can be highly specific as they do not bind internal sequences (Edwards et al., 1995). Models of the tertiary structures of mammalian P450s predict that the C-termini of these enzymes are accessible in the native proteins and are not membrane bound (Nelson and Strobel, 1989; Edwards et al., 1991). Edwards et al. directionally conjugated 5-residue peptides representing the C-termini of different $\mathrm{P} 450$ proteins to carrier protein and successfully generated specific antibodies. The last 5 residues for the CYP2Ns and the CYP2Ps are:

CYP2N1 SAKAR

CYP2N2 SAKIR

CYP2P1

CYP2P2 CATPR

CYP2P3 CAVPR

Blast searches with these peptides found no matches in the protein databases suggesting that CYP2N and CYP2P specific antibodies might be generated with these peptides.

Alternatively, antigenicity plots (MacVector) may identify specific internal epitopes that could be used to generate peptide antibodies. Although antigenicity plots do not identify any obvious isoform- or subfamily-specific epitopes in the CYP2N and CYP2P deduced amino acid sequences, a more detailed search might identify subfamily specific epitopes. 
In order to identify the specific cell types that express CYP2N and CYP2P transcripts, in situ hybridization was attempted. The basic protocol was based on a protocol developed by Koji and Nakane using thymine-thymine dimerized DNA as a nonradioactive probe (Koji and Nakane, 1990; Koji and Nakane, 1996). The goal was to develop a protocol compatible to the immunohistochemistry protocols and kits already in use in the laboratory.

The probes were gene specific and were based on 5'-non-coding regions of CYP2N1 and CYP2N2. Each probe had TTATTATTA on its 5'-end and ATTATT on its 3'-end. The CYP2N1 probe was 5'-

\section{TTATTATTAAGACCCAAAAAGGCAGCAGCAGAATAATGCCTGTGCCTATCTGGT} ATTATT. The CYP2N2 probe was 5'-

\section{TTATTATTAAGCAGAACTGCAATGGAAGAACTGCCTGTAGTAGTCCAAACTGCC}

ATTATT. These two probes have equal numbers of the different nucleotides, and so the nonsense control probe was the same for both and consisted of these nucleotides randomly arranged. It was $5^{\prime}-$

\section{TTATTATTAGAACTGACTGACTGAACGATACGACGAACGTAACGATCGATCTGC}

ATTATT. Thymine dimers were formed by irradiating the probes with $7 \mathrm{~kJ}$ of ultraviolet light using a Stratalinker (Stratagene).

Fish were sacrificed and tissues were submersed in freshly made $4 \%$ paraformaldehyde/phosphate buffered saline (PFA/PBS), 4 hours at room temperature. The PFA/PBS is made fresh to prevent the formation of formic acid (Singer et al., 1986). Tissues were immediately wax-embedded. 0.4 micron sections were cut and mounted on Superfrost Plus slides (Curtin Matheson Scientific, Inc.), dried overnight, and melted onto the slides to prevent later edge effects. 
The basic protocol is outlined below with brief explanations in parentheses. A number of papers influenced this protocol (Singer et al., 1986; Koji and Nakane, 1990; Koji and Nakane, 1996). The Signet Peroxidase Anti-Peroxidase Detection System is from Signet Laboratories, Dedham, MA. 


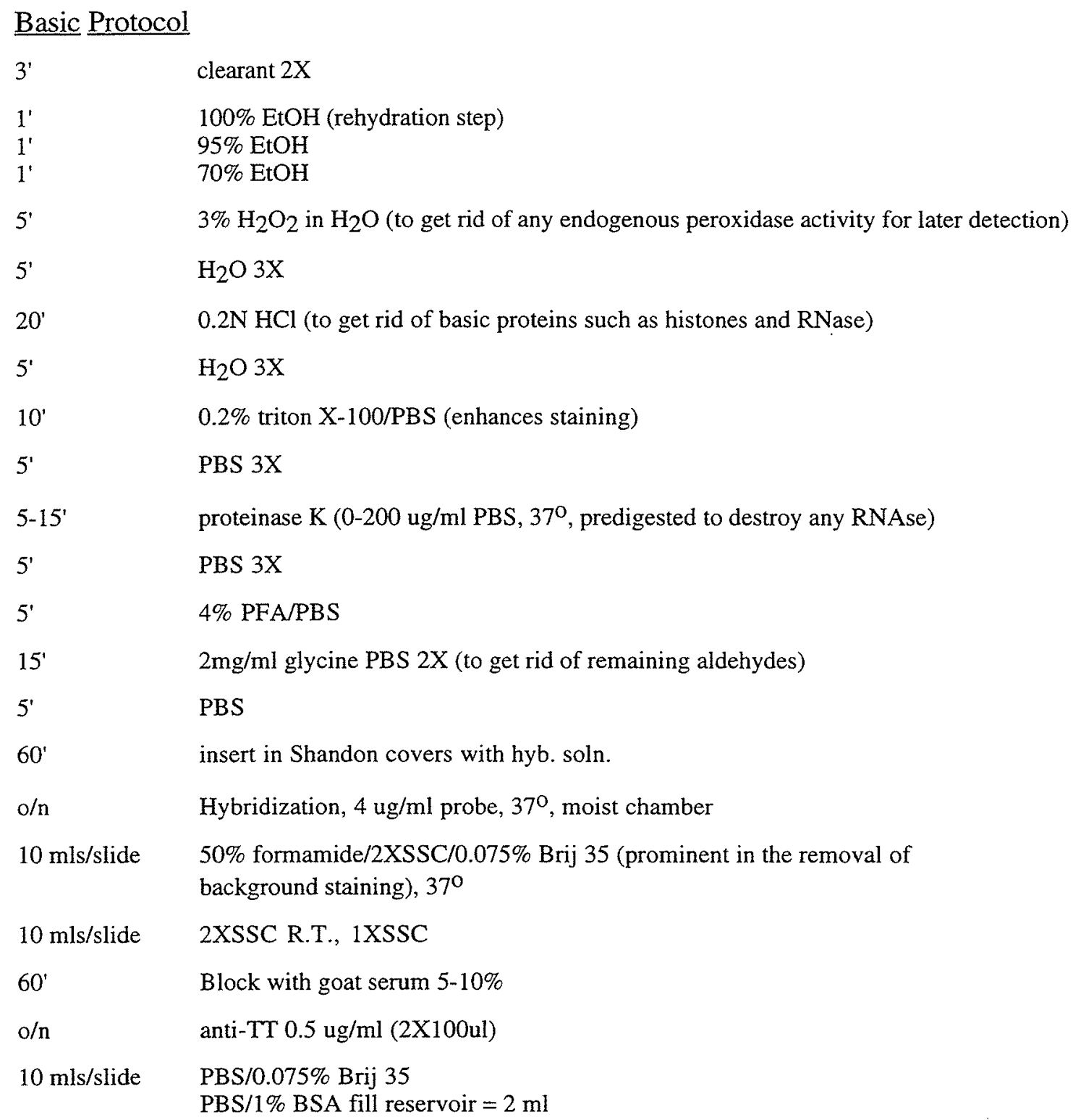

Signet Peroxidase Anti-Peroxidase System

$60^{\prime}$

goat anti-mouse IgG (2X150ul at $\mathrm{t}=0$ and $\left.30^{\prime}\right)$

PBS/0.075\% Brij 35 fill resevoir $=2 \mathrm{ml}$ $\mathrm{PBS} / 1 \% \mathrm{BSA}$ fill reservoir $=2 \mathrm{ml}$

60 peroxidase linked mouse antibody

15 $\quad$ PBS/0.075\% Brij 35 fill resevoir $=2 \mathrm{ml}$

$\mathrm{PBS} / 1 \%$ BSA fill reservoir $=2 \mathrm{ml}$

PBS fill reservoir $=2 \mathrm{ml}$ 
All solutions were treated with DEPC when possible.

Hybridization solution contained: $40 \%$ formamide $10 \mathrm{mM}$ Tris $\mathrm{pH} 7.4$

$0.6 \mathrm{M} \mathrm{NaCl}$ $1 \mathrm{mM}$ EDTA pH 8

$1 \mathrm{X}$ Denhardt's $250 \mathrm{ug} / \mathrm{ml}$ tRNA $125 \mathrm{ug} / \mathrm{ml}$ DNA $10 \%$ dextran sulfate $200 \mathrm{U} / \mathrm{ml}$ heparin (blocks non-specific binding of probes to protein) $0.01 \%$ SDS (decreases non-specific staining) $4 \mathrm{ug} / \mathrm{ml}$ probe dimerized with $7000 \mathrm{~J}$ uv

Block solution contained: $\quad 5 \%$ BSA in PBS

$100 \mathrm{ug} / \mathrm{ml}$ DNA

$100 \mathrm{ug} / \mathrm{ml}$ tRNA

$0.1 \%$ SDS

$0.05 \% \mathrm{NaN}_{3}$

$5 \%$ goat serum

Specific binding with these probes was not seen. A variety of changes might be made in the permeabilization step. Digestion with proteinase $\mathrm{K}$ still needs to be optimized. Each tissue should be processed separately (originally all tissues were embedded in the same paraffin block) since each requires a different period of digestion. Other methods to permeabilize the tissues also were tried. One method included microwave-treating the slides in $10 \mathrm{mM}$ sodium citrate $\mathrm{pH} 6$ for various periods of time (Lan et al., 1996). Pronase also was tried instead of proteinase $\mathrm{K}$. Another method used different concentrations of saponin (Yamawaki et al., 1993). Saponins are naturally occurring, water soluble, plant glycosides that bind to cholesterol and other $\beta$-hydroxysteroids, permeabilizing the membrane without destroying the tissue morphology (Bangham and Horne, 1962). Ideally, this treatment would enable both in situ histochemical as well as immunohistochemical techniques to be performed on the same tissue section. These were tried alone and in combination with each other and with proteinase $\mathrm{K}$. 
Other labels and/or probes could be tried. Biotin and dioxigenin could be used to label the probes rather than thymine dimers. Riboprobes also might be tried. Although more difficult to work with than DNA probes, they often give good results. A variety of companies sell kits to synthesize non-radioactive riboprobes for use with in situ hybridization.

Controls include probing ribosomal RNA as a reference for hydbridizable RNA in tissue sections. This is particularly important when the initial tissue fixation protocol is unknown or different between samples. RNAse treatment needs to be done to ensure that RNA is hybridizing. Unlabeled probe in excess plus anti-sense labelled probe, nonhomologous unlabeled in excess plus anti-sense labelled probe, sense probe, and nonsense probe also need to be used to ensure specific binding of the anti-sense probe. 


\section{BIBLIOGRAPHY}

Adesnik, M., Bar-Nun, S., Maschio, F., Zunich, M., Lippman, A. and Bard, E. (1981). Mechanism of induction of cytochrome P-450 by phenobarbital. J. of Biol. Chem. 256: 10340-10345.

Akira, S., Isshiki, H., Sugita, T., Tanabe, O., Kinoshita, S., Nishio, Y., Nakajima, T., Hirano, T. and Kishimoto, T. (1990). A nuclear factor for IL-6 expression (NF-IL6) is a member of a C/EBP family. EMBO J 9: 1897-1906.

Andersson, T., Pesonen, M. and Johansson, C. (1985). Differential induction of cytochrome P-450-dependent monooxygenase, epoxide hydrolase, glutathione trnasferase, and UDP glucuronyltransferase activities in the liver of the rainbow trout by Bnaphthoflavone or Clophen A50. Biochem. Pharm. 34: 3309-3314.

Aoyama, T., Korzekwa, K., Matsunaga, T., Nagata, K., Gillette, J., Gelboin, H. V. and Gonzalez, F. J. (1990). cDNA-directed expression of rat P450s IIA1 AND IIA2. Catalytic activities toward steroids and xenobiotics and comparison with the enzymes purified from liver. Drug Metab. Disp. 18: 378-382.

Armstrong, P. B. and Child, J. S. (1965). Stages in the normal development of Fundulus heteroclitus. Biol. Bull. 128: 143-168.

Baksi, S. M. and Frazier, J. M. (1990). Isolated fish hepatocytes - model systems for toxicology research. Aquat. Toxicol. 16: 229-256.

Bangham, A. D. and Horne, R. W. (1962). Action of saponin on biological cell membranes. Nature 196: 952.

Bell, J. G., Tocher, D. R., Macdonald, F. M. and Sargent, J. R. (1995). Diets rich in eicosapentaenoic acid and gamma-linolenic acid affect phospholipid fatty acid composition and production of prostaglandins $\mathrm{E}(1), \mathrm{E}(2)$ and $\mathrm{E}(3)$ in turbot (Scophthalmus maximus), a species deficient in Delta 5 fatty acid desaturase. Prostagland Leuk Essent Fatty 53: 279286.

Bernardi, G., Sordino, P. and Powers, D. A. (1993). Concordant Mitochondrial and Nuclear DNA Phylogenies for Populations of the Teleost Fish Fundulus-heteroclitus. Proc Natl Acad Sci USA 90: 9271-9274.

Bigelow, H. B. and Schroeder, W. C. (1953). Fishes of the Gulf of Maine. Washington, United States Government Printing Office.

Black, S. D. and Coon, M. J. (1987). P-450 cytochromes: Structure and function. Adv. Enzymol. 60: 35-87.

Bornheim, L. M., Underwood, M. C., Caldera, P., Rettie, A. E., Trager, W. F., Wrighton, S. A. and Correia, M. A. (1987). Inactivation of multiple hepatic cytochrome P450 isozymes in rats by allylisopropylacetamide: mechanistic implications. Mol. Pharmacol. 32: 299-308. 
Boyle, S. and James, M. O. (unpublished data). CYP2L2.

Breathnach, R. and Chambon, P. (1981). Organization and expression of eukaryotic split genes coding for proteins. Annu. Rev. Biochem. 50: 349-383.

Brenner, S., Elgar, G., Sandford, R., Macrae, A., Venkatesh, B. and Aparicio, S. (1993). Characterization of the pufferfish (Fugu) genome as a compact vertebrate genome. Nature 366: 265-268.

Buhler, D. R. (unpublished data). CYP2K3.

Buhler, D. R. (unpublished data). CYP3A27.

Buhler, D. R., Yang, Y. H., Dreher, T. W., Miranda, C. L. and Wang, J. L. (1994). Cloning and sequencing of the major rainbow trout constitutive cytochrome $\mathrm{P} 450$ (CYP2K1): identification of a new cytochrome P450 gene subfamily and its expression in mature rainbow trout liver and trunk kidney. Arch. Biochem. Biophys. 312: 45-51.

Capdevila, J., Kim, Y. R., Martin-Wixtrom, C., Falck, J. R., Manna, S. and Estabrook, R. W. (1985). Influence of a fibric acid type of hypolipidemic agent on the oxidative metabolism of arachidonic acid by liver microsomal cytochrome P-450. Arch. Biochem. Biophys. 243: 8-19.

Capdevila, J., Marnett, L. J., Chacos, N., Prough, R. A. and Estabrook, R. W. (1982). Cytochrome P-450-dependent oxygenation of arachidonic acid to hydroxyicosatetraenoic acids. Proc. Natl. Acad. Sci. USA 79: 767-770.

Capdevila, J., Snijder, G. D. and Falck, J. R. (1984). Epoxygenation of arachidonic acid by rat anterior pituitary microsomal fractions. FEBS Lett. 178: 319-322.

Capdevila, J. H., Chacos, N., Werringloer, J., Prough, R. A. and Estabrook, R. W. (1981). Liver microsomal cytochrome P450 and the oxidative metabolism of arachidonic acid. Proc. Natl. Acad. Sci. U.S.A. 78: 7375-7378.

Capdevila, J. H., Dishman, E., Karara, A. and Falck, J. R. (1991). Cytochrome P450 arachidonic acid epoxygenase: stereochemical characterization of epoxyeicosatrienoic acids. Meth. Enzymol. 206: 441-53.

Capdevila, J. H., Falck, J. R., Dishman, E. and Karara, A. (1990). Cytochrome P450 arachidonate oxygenase. Meth. Enzymol. 187: 385-394.

Capdevila, J. H., Jin, Y., Karara, A. and Falck, J. R. (1993). Cytochrome P450 epoxygenase dependent formation of novel endogenous epoxyeicosatrienoyl phospholipids. Eicosanoids and Other Bioactive Lipids in Cancer, Inflammation and Radiation Injury. Boston, MA, Klumer Academic Publishers. 11-15.

Capdevila, J. H., Pramanik, B., Napoli, J. L., Manna, S. and Falck, J. R. (1984). Arachidonic acid epoxidation: epoxyeicosatrienoic acids are endogenous constituents of rat liver. Arch. Biochem. Biophys. 231: 511-517. 
Capdevila, J. H., Pranamik, B., Napoli, J. L., Manna, S. and Falck, J. R. (1986). Arachidonic acid epoxidation: epoxyeicostrienoic acids as endogenous constituents of rat liver. Arch. Biochem. Biophys. 231: 511-517.

Capdevila, J. H., Zeldin, D. C., Karara, A. and Falck, J. R. (1996). Eicosanoid metabolism and bioactivation by microsomal cytochrome P450. Advances in Molecular and Cell Biology. JAI Press, Inc. 317-339.

Capdevila, J. R., Yadagiri, P., Manna, S. and Falck, J. R. (1986). Absolute configuration of the hydroxyeicosatrienoic acids (HETEs) formed during catalytic oxygenation of arachidonic acid by microsomal cytochrome P450. Biochem. Biophys. Res. Commun. 141: 1007-1011.

Celander, M., Buhler, D. R., Forlin, L., Goksoyr, A., Miranda, C. L., Woodin, B. R. and Stegeman, J. J. (1996). Immunochemical relationships of cytochrome P4503A-like proteins in teleost fish. Fish Physiology and Biochemistry 15: 323-332.

Celander, M., Ronis, M. and Forlin, L. (1989). Initial characterization of a constitutive cytochrome P-450 isoenzyme in rainbow trout liver. Mar. Environ. Res. 28: 9-13.

Celander, M. and Stegeman, J. J. (1997). Isolation of a cytochrome P450 3A cDNA sequence (CYP3A30) from the marine teleost Fundulus heteroclitus and phylogenetic analyses of CYP3A genes. Biochem. Biophys. Res. Com. 236: 306-312.

Chang, X. T., Kobayashi, T., Nakamura, M., Kajura, H. and Nagahama, Y. (1996). Isolation and characterization of cDNA encoding the tilapia (Oreochromis niloticus) cytochrome $\mathrm{P} 450$ aromatase (P450arom): changes in P450arom mRNA, protein and enzyme activity in ovarian follicles during oogenesis. J. Mol. Endocrinol. in press:

Chomczynski, P. (1992). One-hour downward alkaline capillary transfer for blotting of DNA and RNA. Analytical Biochemistry 201: 134-139.

Cinti, D. L. and Feinstein, M. B. (1976). Platelet cytochrome P450: a possible role in arachidonate-induced aggregation. Biochem. Biophys. Res. Commun. 73: 171-179.

Clare, R. A., Huang, S., Doig, M. V. and Gibson, G. G. (1991). Gas chromatographicmass spectrometric characterisation of some novel hydroxyeicosatetraenoic acids formed on incubation of arachidonic with microsomes from induced rat livers. J. Chromatography 562: $237-247$.

Corey, E. J., Marfat, A., Falck, J. R. and Albright, J. O. (1980). Controlled chemical synthesis of the enzymatically produced eicosanoids 11-, 12-, and 15-HETE from arachidonic acid and conversion into the corresponding hydroperoxides (HPETE). J. Am. Chem. Soc. 102: 1433-1435.

Crawford, M. A. and Harbige, L. S. (1988). n-3 fatty acids and the evolution of the brain. Biological Membranes: Abberations in Membrane Structure and Function. Proceedings of the 12th International Conference on Biological Membranes. New York, Alan R. Liss, Inc. 335-354. 
D'Avanzo, C. and Valiela, I. (1990). Use of detrital foods and assimilation of nitrogen by coastal detritivores. Estuaries 13: 20-24.

Destephano, D. B. and Brady, U. E. (1977). Prostaglandin and prostaglandin synthetase in the cricket, Acheta domesticus. J. Insect Physiol. 23: 905-911.

Ding, X. and Coon, M. J. (1990). Immunochemical characterization of multiple forms of cytochrome P-450 in rabbit nasal microsomes and evidence for tissue-specific expression of P450s NMa and NMb. Mol. Pharm. 37: 489-496.

Ding, X., Porter, T. D., Peng, H.-W. and Coon, M. J. (1991). cDNA and derived amino acid sequence of rabbit nasal cytochrome P450NMb (P450חG1), a unique isozyme possibly involved in olfaction. $285: 120-125$.

Edwards, R. J., Murray, B. P., Singleton, A. M. and Boobis, A. R. (1991). Orientation of cytochromes P450 in the endoplasmic reticulum. Biochemistry 30: 71-6.

Edwards, R. J., Singleton, A. M., Murray, B. P., Davies, D. S. and Boobis, A. R. (1995). Short synthetic peptides exploited for reliable and specific targeting of antibodies to the C-termini of cytochrome P450 enzymes. Biochem Pharmacol 49: 39-47.

Elskus, A. A. and Stegeman, J. J. (1989). Further consideration of phenobarbital effects on cytochrome P-450 activity in the killfish, Fundulud heteroclitus. Comp. Biochem. Physiol. 92C: 223-230.

Elskus, A. A. and Stegeman, J. J. (1989). Induced cytochrome P-450 in Fundulus heteroclitus associated with environmental contamination by polychlorinated biphenyls and polynuclear aromatic hydrocarbons. Mar. Environ. Res. 27: 31-50.

Escalante, B., Erlij, D., Falck, J. R. and McGiff, J. C. (1991). Effect of cytochrome P450 arachidonate metabolites on ion transport in rabbit kidney Loop of Henle. Science 251: 799-802.

Escalante, B., Falck, J. R., Yadagiri, P., Sun, L. and Laniado-Schwartzman, M. (1988). 19(S)-hydroxyeicosatetraenoic acid is a potent stimulator of renal Na+-K+-ATPase. Biochem. Biophys. Res. Comm. 152: 1269-1274.

Evans, C. T., Corbin, C. J., Saunders, C. T., Merril, J. C., Simpson, E. R. and Mendelson, C. R. (1987). Regulation of estrogen biosynthesis by human adipose stromal cells: effects of dibutyryl cyclic AMP, epidermal growth factor, and phorbol ester on the synthesis of aromatase cytochrome P450. Journal of Biological Chemistry 262: 69146920.

Falck, J. R., Lumin, S., Blair, I., Dishman, E., Martin, M. V., Waxman, D. J., Guengerich, F. P. and Capdevila, J. H. (1990). Cytochrome P450-dependent oxidation of arachidonic acid to 16-, 17-, and 18-hydroxyeicosatetraenoic acids. J. Biol. Chem. 265: 10244-10249.

Falck, J. R. and Manna, S. (1982). 8,9-epoxyarachidonic acid: a cytochrome P-450 metabolite. Tetrahedron Lett. 23: 1755-1756. 
Falck, J. R., Yagagiri, P. and Capdevila, J. H. (1990). Synthesis of epoxyeicosatrienoic acids and heteroatom analogs. Meth. Enzymol. 187: 357-364.

Falckh, P. H. J., Wu, Q. K. and Ahokas, J. P. (1997). CYP4T1-a cytochrome P450 expressed in rainbow trout (Oncorhynchus mykiss) liver. Biochem. Biophys. Res. Com. 236: 302-305.

Faulkner, D. J. and Fenical, W. H., Ed. (1977). Marine Natural Products Chemistry. New York, Plenum Press.

Felsenstein, J. (1985). Confidence limits on phylogenies: an approach using the bootstrap. Evolution 39: 783-791.

Fischer, S. M. and Slaga, T. J., Ed. (1985). Arachidonic Acid Metabolism and tumor promotion. Boston, Martinus Nijhoff.

Fitzgerald, L. M., Rodrigues, A. and Smutzer, G. (1993). Codon usage in bony fishes. Molecular Marine Biology and Biotechnology 2(2): 112-119.

Fitzpatrick, F., Ennis, M., Baze, M., Wynalda, M., McGee, J. and Liggett, W. (1986). Inhibition of cyclooxygenase activity and platelet aggregation by epoxyeicosatrienoic acids: influence of stereochemistry. J. Biol. Chem. 261: 15334-15338.

Fitzpatrick, F. A. and Murphy, R. C. (1989). Cytochrome P450 metabolism of arachidonic acid: formation and biological actions of "epoxygenase"-derived eicosanoids. Pharmacol. Rev. 40: 229-241.

Ford-Hutchinson, A. W., Gresser, M. and Young, R. N. (1994). 5-lipoxygenase. Annu. Rev. Biochem. 63: 383-417.

Freas, W. and Grollman, S. (1980). Ionic and osmotic influences on prostaglandin release from the gill tissue of a marine bivalve, Modiolus demissus. J. Exp. Biol. 84: 169-185.

Fulco, A. J., He, J. S. and Liang, Q. W. (1994). The role of conserved 5'-flanking (Barbie Box) DNA sequences and barbiturate-responsive DNA-binding proteins in the mechanism of induction by barbiturates of $\mathrm{P} 450$ cytochromes in Bacillus megaterium and other prokaryotic and eukaryotic organisms. Cytochrome P450. 37-42.

Funk, C. D. (1993). Molecular biology in the eicosanoid field. Progress in Nucleic Acid Research and Molecular Biology 45: 67-98.

Gebremedhin, D., Ma, Y.-H., Falck, J. R., Roman, R. J., VanRollins, M. and Harder, D. (1992). Mechanism of action of cerebral epoxyeicosatrienoic acids on cerebral arterial smooth muscle. Am. J. Physiol. H519-H525.

Gelinas, D. M., Pitoc, G. A. and Callard, G. V. (1996). Isolation of goldfish brain aromatase cDNA and analysis of expression during the reproductive cycle and after steroid treatment in vivo. Unpublished

Gibson, G. G. (1989). Comparative aspects of the mammalian P450 IV gene family. 19: 123-148. 
Gilday, D., Gannon, M., Yutzey, K., Bader, D. and Rifkind, A. B. (1996). Molecular cloning and expression of two novel avian cytochrome P4501A enzymes induced by 2,3,7,8-tetrachlorodibenzo-p-dioxin. J. Biol. Chem. 271: 33054-.

Goetz, F. W. (1990). Compartmentalization of prostaglandin synthesis within the fish ovary. Am. J. of Physiol. 29: R862-R865.

Gong, B., Townley, R. and Farrell, A. P. (1997). Effects of polyunsaturated fatty acids and some of their metabolites on mitotic activity of vascular smooth muscle explants from the coronary artery of rainbow trout (Oncorhynchus mykiss). Can. J. Zool. 75: 80-86.

\section{Gong, Z. (1994). CYP52. unpublished data}

Gonzalez, F. J. (1989). The molecular biology of cytochrome P-450s. Pharmacol. Rev. 40: 243-287.

Gonzalez, F. J., Kimura, S., Tamura, S. and Gelboin, H. V. (1991). Expression of mammalian cytochrome P450 using baculovirus. Methods Enzymol 206: 93-99.

Gonzalez, F. J. and Nebert, D. W. (1990). Evolution of the P450 gene superfamily. Trends Genet. 6: 182-186.

Gotoh, O. (1992). Substrate Recognition Sites in Cytochrome P450 Family 2 (CYP2) Proteins inferred from Comparative Analyses of Amino Acid and Coding Nucleotide Sequences. Journal of Biological Chemistry 267: 83-90.

Graf, H., Ruf, H. H. and Ulrich, V. (1983). Prostacyclin synthase, a cytochrome P450 enzyme. Agnew. Chem. Int. Ed. Engl. 22: 487-488.

Graves, P. E., Elhag, G. A., Ciaccio, P. J., Bourque, D. P. and Halpert, J. R. (1990). cDNA and deduced amino acid sequences of a dog hepatic cytochrome P450IIB responsible for the metabolism of $2,2^{\prime}, 4,4^{\prime}, 5,5^{\prime}$-hexachlorobiphenyl. Arch. Biochem. Biophys. 281: 106-115.

Gray, E. S., Woodin, B. R. and Stegeman, J. J. (1991). Sex differences in hepatic monooxygenases in winter flounder (Pseudopleuronectes americanus) and scup (Stenotomus chrysops) and regulation of P450 forms by estradiol. J. Exper. Zool. 259: 330-342.

Guengerich, F. P. and D C, L. (1985). Enzymatic activation of chemicals to toxic metabolites. CRC Crit Rev Toxicol 14: 259-307.

Hahn, M. E., Lamb, T. M., Schultz, M. E., Smolowitz, R. M. and Stegeman, J. J. (1993). Cytochrome P4501A induction and inhibition by 3,3',4,4'-tetrachlorobiphenyl in an Ah receptor-containing fish hepatoma cell line (PLHC-1). Aquat. Toxicol. 26: 185-208.

Hahn, M. E. and Stegeman, J. J. (1994). Regulation of cytochrome P4501A1 in teleosts: sustained induction of CYP1A1 mRNA, protein, and catalytic activity by $2,3,7,8$ tetrachlorodibenzofuran in the marine fish Stenotomus chrysops. Toxicol. Appl. Pharmacol. 127: 187-198. 
Hammonds, T. D., Blair, I. A., Falck, J. R. and Capdevila, J. H. (1989). Resolution of epoxyeicosatrienoate enantiomers by chiral phase chromatography. Anal. Biochem. 182: 300-303.

Hankinson, O. (1995). The aryl hydrocarbon receptor complex. Annual Review of Pharmacology and Toxicology 35: 307-340.

Hansen, A. and May, B. (1989). Sequence of chicken phenobarbital-inducible P450 cDNA: regulation of two P450 mRNAs transcribed from different genes. DNA 8: 179191.

Harder, D. R., Campbell, W. B. and Roman, R. J. (1995). Role of cytochrome P-450 enzymes and metabolites of arachidonic acid in the control of vascular tone. J. Vasc. Res. 32: $79-92$.

Haurand, M. and Ullrich, V. (1985). Isolation and characterization of thromboxane synthase from human platelets as a P-450 enzyme. J. Biol. Chem. 260: 15059-15067.

Higgins, D. G. and Sharp, P. M. (1988). CLUSTAL: a package for performing multiple sequence alignment on a microcomputer. Gene 73: 237-244.

Hightower, L. E. and Renfro, J. L. (1988). Recent applications of fish cell culture to biomedical research. J. Experim. Zool. 248: 290-302.

Hinegardner, R. and Rosen, D. E. (1972). Cellular DNA content and the evolution of teleostean fishes. American Naturalist 106: 621-644.

Hobbs, A. A., Mattschoss, L. A., May, B. K., Williams, K. E. and Elliot, W. H. (1986). The cDNA and protein sequence of a phenobarbital-induced chicken cytochrome P-450. $J$. Biol. Chem. 261: 9444-9449.

Hood, L., Campbell, J. H. and Elgin, S. C. R. (1975). The organization, expression, and evolution of antibody genes and other multigene families. Annual Rev. Genetics 305-353.

Horn, M. H. (1989). Biology of Marine Herbivorous Fishes. Oceanogr. Mar. Biol. Annual Review 27: 167-272.

Hsiao, S.-M. and Meier, A. H. (1989). Comparison of semilunar cycles of spawning activity in Fundulus grandis and $F$. heteroclitus held under constant laboratory conditions. Journal of Experimental Zoology 252: 213-218.

Hsiao, S. M., Greeley, M. S. and Wallace, R. A. (1994). Reproductive cycling in female Fundulus heteroclitus. Biol Bull 186: 271-284.

Isselbacher, K. J., Braunwald, E., Wilson, J. D., Martin, J. B., Fauci, A. S. and Kasper, D. L., Ed. (1994). Harrison's Principles of Internal Medicine. New York, McGraw-Hill, Inc.

Jaffe, L. F. and Nuccitelli, R. (1974). An ultrasensitive vibrating probe for measuring steady extracellular currents. J. Cell Biol. 63: 614-628. 
James, M. O., Boyle, S. M., Trapido-Rosenthal, H. G., Carr, W. E. S. and Shiverick, K. $T$. (1993). Identification of a cytochrome P450 sequence in cDNA from the hepatopancreas of the florida spiny lobster. FASEB J. 7: A1201.

James, M. O., Boyle, S. M., Trapido-Rosenthal, H. G., Smith, W. C., Greenberg, R. M. and Shiverick, K. T. (1996). cDNA and protein sequence of a major form of P450, CYP2L, in the hepatopancreas of the spiny lobster, Panulirus argus. Arch. Biochem. Biophys. 329: 31-38.

Junier, M.-P., Dray, F., Blair, I., Capdevila, J., Dishman, E., Falck, J. R. and Ojeda, S. R. (1990). Epoxygenase products of arachidonic acid are endogenous constituents of the hypothalamus involved in D2 receptor-mediated, dopamine-induced release of somatostatin. Endocrinology 126: 1534-1540.

Kaplan, L. A. E., Schultz, M. E., Schultz, R. J. and Crivello, J. F. (1991). Nitrosodiethylamine metabolism in the viviparous fish Poeciliopsis : Evidence for the existence of liver P450pj activity and expression. Carcinogenesis 12: 647-652.

Karara, A., Dishman, E., Blair, I., Falck, J. R. and Capdevila, J. H. (1989). Endogenous epoxyeicosatrienoic acids. Cytochrome P-450 controlled stereoselectivity of the hepatic arachidonic acid epoxygenase. J. Biol. Chem. 264: 19822-7.

Keller, H., Dreyer, C., Medin, J., Mahfoudi, A., Ozato, K. and Wahli, W. (1993). Fatty acids and retinoids control lipid metabolism through activation of peroxisome proliferatoractivator receptor-retinoid X receptor heterodimers. Proc Natl Acad Sci USA 90: 2160 2164.

Kerry, N. L., Somogyi, A. A., Bochner, F. and Mikus, G. (1994). The role of CYP2D6 in primary and secondary oxidative metabolism of dextromethorphan: In vitro studies using human liver microsomes. Br J Clin Pharmacol 38: 243-248.

Kikuta, Y., Sogawa, K., Haniu, M., Kinosaki, M., Kusunose, E., Nojimas, Y., Yamamoto, S., Ichihara, K., Kusunose, M. and Fujii-Kuriyama, Y. (1991). A novel species of cytochrome $\mathrm{P}-450\left(\mathrm{P}_{450} \mathrm{ib}_{\mathrm{b}}\right)$ specific for the small intestine of rabbits. cDNA cloning and its expression in COS cells. J. Biol. Chem. 266: 17821-17825.

Kim, J. B., Spotts, G. D., Halvorsen, Y.-D., Shih, H.-M., Ellenberger, T., Towle, H. C. and Spiegelman, B. M. (1995). Dual DNA binding specificity of ADD1/SREBP1 controlled

by a single amino acid in the basic helix-loop-helix domain. Mol. Cell. Biol. 15: 25822588 .

Kleinow, Melancon, K. M. and Lech, J. (1987). Biotransformation and induction: implications for toxicity, bioaccumulation and monitoring of environmental xenobiotics in fish. Environ. Health Perspect. 61: 105-119.

Kleinow, K. M., Haasch, M. L., Williams, D. E. and Lech, J. J. (1990). A comparison of hepatic P450 induction in rat and trout (Oncorhynchus mykiss ): Delineation of the site of resistance of fish to phenobarbital-type inducers. Comp. Biochem. Physiol. C 96C: 259-270. 
Kloepper-Sams, P. J., Park, S. S., Gelboin, H. V. and Stegeman, J. J. (1987). Specificity and cross-reactivity of monoclonal and polyclonal antibodies against cytochrome P450E of the marine fish scup. Arch. Biochem. Biophys. 253: 268-278.

Kloepper-Sams, P. J. and Stegeman, J. J. (1989). The temporal relationships between cytochrome P-450E protein content, catalytic activity and mRNA levels in the teleost Fundulus heteroclitus following treatment with B-naphthoflavone. Arch. Biochem. Biophys. 268: 525-535.

Kobayashi, D., Matsuyama, M., Tanaka, M., Fukada, S. and Nagahama, Y. (1996). Structural analysis of medaka P-450c17 and expression in the ovarian follicle. Unpublished

Koji, T. and Nakane, P. K. (1990). Localization in situ of specific mRNA using thyminethymine dimerized DNA probes. Acta Pathologica Japonica 40: 793-807.

Koji, T. and Nakane, P. K. (1996). Recent advances in molecular histochemical techniques: in situ hybridization and southwestern histochemistry. J Electron Microsc 45: 119-127.

Koop, D. R. (1992). Oxidative and reductive metabolism by cytochrome P450 2E1. FASEB Journal 6: 724-730.

Korzekwa, K. and Jones, J. (1993). Predicting the cytochrome P450 mediated metabolism of xenobiotics. Pharmacogenetics 3: 1-18.

Kozak, M. (1991a). Structural features in eukaryotic mRNAs that modulate the initiation of translation. Journal of Biological Chemistry 266: 19867-19870.

Kozak, M. (1991b). An analysis of vertebrate mRNA sequences: intimations of translational control. Journal of Cell Biology 115: 887-903.

Kraft, A. S. and Anderson, W. B. (1983). Phorbol esters increase the amount of Cat+, phospholipid-dependent protein kinase associated with plasma membrane. Nature 301: 621-623.

Krey, G., Braissant, O., L'Horset, F., Kalkhoven, E., Perroud, M., Parker, M. G. and Wahli, W. (1997). Fatty acid, eicosanoids, and hypolipidemic agents identified as ligands of peroxisome proliferator-activated receptors by coactivator-dependent receptor ligand assay. Mol. Endocrinol. 11: 779-791.

Kunsch C., R. S. M., Rosen C. A. (1992). Selection of optimal kappaB/Rel DNA-binding motifs:

interaction of both subunits of NF-kappaB with DNA is required for transcriptional activation. Mol. Cell. Biol. 12: 4412-4421.

Laethem, R. M., Balazy, M., Falck, J. R., Laethem, C. L. and Koop, D. R. (1993).

Formation of $19(\mathrm{~S})-, 19(\mathrm{R})$, and 18(R)-hydroxyeicosatetraenoic acids by alcohol-inducible cytochrome P450 2E1. J. Biol. Chem. 268: 12912-12918. 
Laethem, R. M., Laethem, C. L. and Koop, D. R. (1992). Purification and properties of a cytochrome $\mathrm{P} 450$ arachidonic acid epoxygenase from rabbit renal cortex. J Biol Chem 267: 5552-9.

Lam, B. K. and Wong, P. Y.-K. (1988). Biosynthesis and biological activities of lipoxin A5 and B5 from eicosapentaenoic acid. Adv. Exp. Med. Biol. 229: 51.

Lan, H. Y., Mu, W., Ng, Y. Y., Nikolic-Paterson, D. J. and Atkins, R. C. (1996). A simple, reliable, and sensitive method for nonradioactive in situ hybridization: use of microwave heating to improve hybridization efficiency and preserve tissue morphology. Journal of Histochemistry and Cytochemistry 44: 281-287.

Latchman, D. S. (1991). Eukaryotic transcription factors. San Diego, Academic Press Limited.

Lauder, G. V. and Liem, K. F. (1983). The evolution and interrelationships of the actinopterygian fishes. Bull. Mus . Comp. Zool. 150: 95-197.

Leaver, M. J., Pirrit, L. and George, S. G. (1993). Cytochrome P450 1A1 cDNA from plaice (Pleuronectes platessa) and induction of P450 1A1 mRNA in various tissues by 3methylcholanthrene and isosafrole. Mol. Mar. Biol. Biotechnol. 2: 338-345.

Lee, C., Kadwell, S. H., Kost, T. A. and Serabjit-Singh, C. J. (1995). CYP3A4 expressed by insect cells infected with a recombinant baculovirus containing both CYP3A4 and human NADPH-cytochrome $\mathrm{P} 450$ reductase is catalytically similar to human liver microsomal CYP3A4. Arch. Biochem. Biophys. 319: 157-167.

Liang Q., H. J.-S., Fulco A. J. (1995). The role of Barbie box sequences as cis-acting elements

involved in the barbiturate-mediated induction of

cytochrome P450BM-1 and P450BM-3 in Bacillus megaterium. J. Biol. Chem. 270: 44384450 .

Liggins, G. C., Fairclough, R. J. and Grieves, S. A. (1973). The mechanism of initiation of parturation in the ewe. Recent Prog. Horm. Res. 29: 111-149.

Lindberg, R. L. P. and Negishi, M. (1989). Alteration of mouse cytochrome P450 substrate specificity by mutation of a single amino acid residue. Nature 339: 632-634.

Lotrich, V. A. (1975). Summer home range and movements of Fundulus heteroclitus (Family: Cyprinonodontidae) in a tidal creek. Ecology 56: 191-198.

Lu, A. Y. H. and Coon, M. J. (1968). Role of hemoprotein P-450 in fatty acid whydroxylation in a soluble enzyme system from liver microsomes. J. Biol. Chem. 243: 1331-1332.

Lu, A. Y. H., Junk, K. W. and Coon, M. J. (1969). Resolution of the cytochrome P450containing w-hydroxylation system of liver microsomes into three components. J. Biol. Chem. 244: 3714-3721. 
Macica, C., Balazy, M., Falck, J. R., Mioskowski, C. and Carroll, M. A. (1993). Characterization of cytochrome P-450-dependent arachidonic acid metabolism in rabbit intestine. Am. J. Physiology G735-G741.

Manna, S., Falck, J. R., Chacos, N. and Capdevila, J. H. (1983). Synthesis of arachidonic acid metabolites produced by purified kidney cortex microsomal cytochrome P450. Tetrahedron Lett. 24: 33-36.

Mansuy, D., Battioni, P. and Battioni, J. P. (1989). Chemical model systems for drugmetabolizing cytochrome-P-450-dependent monooxygenases. Eur. J. Biochem. 184: 267285.

Matsutani, T. and Nomura, T. (1987). In vitro effects of serotonin and prostaglandins on release of eggs from the ovary of the scallop, Patinopecten yessoensis. Gen. Comp. Endocrinol. 67: 111-118.

McGiff, J. C. (1991). Cytochrome P450 metabolism of arachidonic acid. Ann. Rev. Pharmacol. Toxicol. 31: 339-69.

Meijer, L., Brash, A. R., Bryant, R. W., Ng, K., Maclouf, J. and Sprecher, H. (1986). Stereospecific induction of starfish oocyte maturation by (8R)-hydroxyeicosatetraenoic acid. J. Biol. Chem. 261: 17040-17047.

Miller, M. R., Hinton, D. E., Blair, J. J. and Stegeman, J. J. (1988).

Immunohistochemical localization of cytochrome P450E in liver, gill and heart of scup (Stenotomus chrysops) and rainbow trout (Salmo gairdneri). Mar. Environ. Res. 24: 37 39.

Miranda, C. L., Wang, J.-L., Henderson, M. C. and Buhler, D. R. (1989). Purification and characterization of hepatic steroid hydroxylases from untreated rainbow trout. Arch. Biochem. Biophys. 268: 227-238.

Mizukami, M., Okauchi, M., Ariyoshi, T. and Kito, H. (1994). The isolation and sequence of cDNA encoding a 3-methylcholanthrene-inducible cytochrome P450 from wild red sea bream, Pagrus major. Marine Biol. 120: 343-349.

Moffat, M. P., Ward, C. A., Bend, J. R., Mock, T., Farhangkhoee, P. and Karmazyn, M. (1993). Effects of epoxyeicosatrienoic acids on isolated hearts and ventricular myocytes. Am. J. Physiol. 264: H1154-H1160.

Morrison, A. R. and Pascoe (1981). Metabolism of arachidonic acid through NADPHdependent oxygenase fo renal cortex. Proc. Nat. Acad. Sci. USA 78: 7375-7378.

Morrison, H. G., Oleksiak, M. F., Cornell, N. W., Sogin, M. L. and Stegeman, J. J. (1995). Identification of cytochrome P450 1A (CYP1A) genes from two teleost fish, toadfish (Opsanus tau) and scup (Stenotomus chrysops), and phylogenetic analysis of CYP1A genes. Biochem. J. 308: 97-104.

Mosset, P., Yadagiri, D., Lumin, S., Capdevila, J. H. and Falck, J. R. (1986). Arachidonic acid epoxygenase: total synthesis of both enentiomers of 8,9- and 11,12epoxyeicosatrienoic acid. Tetrahedron Lett. 27: 6035-6038. 
Moustakis, C. A., Viala, J., Capdevila, J. H. and Falck, J. R. (1986). Total synthesis of the cytochrome P-450 epoxygenase metabolites $5(R), 6(S)-, 5(S), 6(R)-$, and $14(R), 15(S)$ epoxyeicosatrienoic acid (EET) and hydration products $5(\mathrm{R}), 6(\mathrm{R})$ - and $14(\mathrm{R}), 15(\mathrm{R})$ dihydroxyeicosatrienoic acid (DHET). J. Am. Chem. Soc. 107: 5283-5285.

Nakai, K., Ward, A. M., Gannon, M. and Rifkind, A. B. (1992). Beta-naphthoflavone induction of a cytochrome P-450 arachidonic acid epoxygenase in chick embryo liver distinct from the aryl hydrocarbon hydroxylase and from phenobarbital-induced arachidonate epoxygenase. J Biol Chem 267: 19503-12.

Nanji, A. A., Zhao, S. P., Sadrzadeh, S. M. H., Dannenberg, A. J., Tahan, S. R. and Waxman, D. J. (1994). Markedly enhanced cytochrome P450 2E1 induction and lipid peroxidation is associated with severe liver injury in fish oil-ethanol-fed rats. Alcohol Clin Exp Res 18: 1280-1285.

Nash, T. (1953). The colorimetric estimation of formaldehyde by means of the Hantzsch reaction. Biochem. J. 55: 416-421.

Nebert, D. W. (1991). Proposed role of drug-metabolizing enzymes: Regulation of steady state levels of the ligands that effect growth, homeostasis, differentiation, and neuroendocrine functions. Mol. Endocrinol. 5: 1202-1214.

Nebert, D. W. (1994). Drug metabolism and signal transduction: possible role of Ah receptor and arachidonic acid cascade in protection from ethanol toxicity. Exs 71: 231-40.

Nef, P., Heldman, J., Lazard, D., Margalit, T., Jaye, M., Hanukoglu, I. and Lancet, D. (1989). Olfactory-specific cytochrome P-450: cDNA cloning of a novel neuroepithelial enzyme possibly involved in chemoreception. 264: 6780-6785.

Nef, P., Larabee, T. M., Kagimoto, K. and Meyer, U. A. (1990). Olfactory-specific cytochrome P-450 (P-450olf1; IIG1). Gene structure and developmental regulation. $J$. of Biol. Chem. 265: 2903-2907.

Nelson, D. and Strobel, H. (1987). Evolution of cytochrome P450 proteins. Mol. Biol. Evol. 4: 572-593.

Nelson, D. R., Kamataki, T., Waxman, D. J., Guengerich, F. P., Estabrook, R. W., Feyereisen, R., Gonzalez, F. J., Coon, M. J., Gunsalus, I. C., Gotoh, O., Okuda, K. and Nebert, D. W. (1993). The P450 Superfamily - Update on New Sequences, Gene Mapping, Accession Numbers, Early Trivial Names of Enzymes, and Nomenclature. DNA Cell Biol. 12: 1-51.

Nelson, D. R., Koymans, L., Kamataki, T., Stegeman, J. J., Feyereisen, R., Waxman, D. J., Waterman, M. R., Gotoh, O., Coon, M. J., Estabrook, R. W., Gunsalus, I. C. and Nebert, D. W. (1996). P450 superfamily: update on new sequences, gene mapping, accession numbers and nomenclature. Pharmacogenetics 6: 1-42.

Nelson, D. R. and Strobel, H. W. (1989). Secondary structure prediction of 52 membrane-bound cytochromes P450 shows a strong structural similarity to P450cam. Bichemistry 28: 656-660. 
Nelson, J. S. (1984). Fishes of the World. New York, John Wiley and Sons.

Nhamburo, P. T., Gonzalez, F. J., McBride, O. W., Gelboin, H. V. and Kimura, S. (1989). Identification of a new P450 expressed in human lung: complete cDNA sequence, cDNA-directed expression, and chromosome mapping. Biochemistry 28: 8060-8066.

Nunez, B. S. and Trant, J. M. (1996). Isolation of the cDNA encoding the interrenal form of cholesterol side chain cleavage cytochrome P450 of the southern stingray (Dasyatis americana). Unpublished

Ohi, H., Sugata, E., Murayama, N. and Higuchi, S. (unpublished data). Cloning and expression analysis of a novel gene for cytochrome P450 in Xenopus laevis.

Oleksiak, M. F., Stegeman, J. J. and etc. (in preparation). Diversity of novel CYP2 genes in Fundulus heteroclitus.

Oleksiak, M. F., Zeldin, D. and Stegeman, J. J. (in preparation). Functional characteristics of novel cytochromes $\mathrm{P} 450$ highly expressed in killifish heart and brain.

Oleksiak, M. F., Zeldin, D. and Stegeman, J. J. (in preparation). In vitro expression of a novel cytochrome P450, CYP2P3: an early vertebrate arachidonic acid catalyst.

Oliw, E. H. (1994). Oxygenation of polyunsaturated fatty acids by cytochrome P450 monooxygenases. Prog. Lipid Res. 33: 329-354.

Oliw, E. H., Guengerich, F. P. and Oates, J. A. (1982). Oxygenation of arachidonic acid by hepatic monooxygenases. Isolation and metabolism of four epoxide intermediates. $J$. Biol. Chem. 257: 3771-3781.

Olsen, G. J. (1988). Phylogenetic analysis using ribosomal RNA. Methods Enzymol 164: 793-812.

Omura, T. and Sato, R. (1964). The carbon monoxide-binding pigment of liver microsomes. J. Biol. Chem. 239: 2370-2378.

Ortiz de Montellano, P. R. (1986). Cytochrome P-450: Structure, Mechanisms, and Biochemistry. N.Y., Plenum.

Parke, D. V., Ioannides, C. and Lewis, D. (1991). The role of the cytochromes P450 in the detoxication and activation of drugs and other chemicals. Can. J. Physiol. Pharm. 69: 537-549.

Pearson, W. (in progress). Protein sequence comparison and protein evolution.

Penczak, T. (1985). Trophic ecology and fecundity of Fundulus heteroclitus in Chezzetcook Inlet, Nova Scotia. Marine Bio. 89: 235-243.

Petzel, D. H. and Stanley-Samuelson, D. W. (1992). Inhibition of eicosanoid biosynthesis modulates basal fluid secretion in the Malpighian tubules of the yellow fever mosquito (Aedes aegypti). J. Insect Physiol. 38: 1-8. 
Porter, N. A., Logan, J. and Konotoyianidou, V. (1979). Preparation and purification of arachidonic acid hydroperoxides of biological importance. J. Org. Chem. 44: 3177-3181.

Powers, D. A., Ropson, I., Brown, D. C., van Beneden, R., Cashon, R., GonzalezVillasenor, L. A. and DiMichele, J. A. (1986). Genetic variation in Fundulus heteroclitus: Geographic distribution. American Zoologist 26: 131-144.

Proctor, K. G., Capdevila, J. H., Flack, J. R., Fitzpatrick, F. A., Mullane, K. M. and McGiff, J. C. (1989). Cardiovascular and renal actions of cytochrome P-450 metabolites of arachidonic acid. Blood Vessels 26: 53-64.

Proctor, K. G., Falck, J. R. and Capdevila, J. (1987). Intestinal vasodialtion by epoxyeicosatrienoic acids: arachidonic acid metabolites produced by a cytochrome P450 monooxygenase. Circ. Res. 60: 50-59.

Quandt, K., Frech, K., Karas, H., Wingender, E. and Werner, T. (1995). Matind and MatInspector - new fast and versatile tools for detection of consensus matches in nucleotide sequence data. Nuc. Acids Res. 23: 4878-4884.

Rifkind, A. B., Kanetoshi, A., Orlinick, J., Capdevila, J. H. and Lee, C. (1994). Purification and biochemical characterization of two major cytochrome P-450 isoforms induced by $2,3,7,8$ tetrachlorodibenzo - p-dioxin in chick embryo liver. $J$ Biol Chem 269: 3387-3396.

Rifkind, A. B., Lee, C., Chang, T. K. H. and Waxman, D. J. (1995). Arachidonic acid metabolism by human cytochrome $\mathrm{P} 450 \mathrm{~s} 2 \mathrm{C} 8,2 \mathrm{C} 9,2 \mathrm{E} 1$, and 1A2: regioselective oxygenation and evidence for a role for CYP2C enzymes in arachidonic acid expoxygenation in human liver microsomes. Arch. Biochem. Biophys. 320: 380-389.

Rowley, A. F., Knight, J., Lloyd-Evans, P., Holland, J. W. and Vickers, P. J. (1995). Eicosanoids and their role in immune modulation in fish-a brief overview. Fish \& Shellfish Immunology 5: 549-567.

Ryan, D., Ramanathan, L., Iida, S., Thomas, P., Haniu, M., Shively, J., Lieber, C. and Levin, W. (1985). Characterization of a major form of rat hepatic microsomal cytochrome P-450 induced by isoniazid. J. Biol. Chem. 260: 6385-6393.

Ryan, D. E., Thomas, P. E. and Levin, W. (1982). Purification and characterization of a minor form of hepatic cytochrome P-450 from rats treated with polychlorinated biphenyls. Arch. Biochem. Biophys. 216: 272-288.

Saitou, N. and Nei, M. (1987). The neighbor-joining method: a new method for reconstructing phylogenetic trees. Mol. Biol. Evol. 4: 406-425.

Sakai, N., Tanaka, M., Adachi, S., Miller, W. L. and Nagahama, Y. (1992). Rainbow trout cytochrome-P-450c17 (17-alpha-Hydroxylase/ 17,20-Lyase) - cDNA cloning, enzymatic properties and temporal pattern of ovarian P-450c17 messenger RNA expression during oogenesis. FEBS Lett 301: 60-64.

Sambrook, J., Fritsch, E. F. and Maniatis, T. (1989). Molecular Cloning: a Laboratory Manual, second edition. Cold Spring Harbor, Cold Spring Harbor Laboratory Press. 
Sanger, F., Nicklen, S. and Coulson, A. R. (1977). DNA sequencing with chainterminating inhibitors. PNAS (USA) 74: 5463-67.

Savas, U., Bhattacharyya, K. K., Christou, M., Alexander, D. L. and Jefcoate, C. R. (1994). Mouse cytochrome P-450EF, representative of a new 1B subfamily of cytochrome P-450s - Cloning, sequence determination and tissue expression. J Biol Chem 269: 1490514911.

Schlenk, D., Ronis, M. J. J., Miranda, C. L. and Buhler, D. R. (1993). Channel catfish liver monooxygenases. Immunological characterization of constitutive cytochromes P450 and the absence of active flavin-containing monooxygenases. Biochemical Pharmacology 45: $217-221$.

Schlezinger, J. J., Zeldin, D. L. and Stegeman, J. J. (in prep). Arachidonic acid metabolism in the marine fish Stenotomus chrysops and the effects of CYP1A induction.

Schuel, H., Moss, R. and Schuel, R. (1985). Induction of polyspermic fertilization in sea urchins by the leukotriene antagonist FPL-55712 and the 5-lipooxygenase inhibitor BW755C. Gamete res. 11: 41-50.

Selman, K. and Wallace, R. A. (1986). Gametogenesis in Fundulus heteroclitus. American Zoologist 26: 173-192.

Sharp, G., Pettitt, T., Rowley, A. and Secombes, C. (1992). Lipoxin-induced migrationof fish leukocytes. Journal of leukocyte Biology 51: 140-145.

Shaw, G. and Kamen, R. (1986). A conserved AU sequence from the 3' untranslated region of GM-CSF mRNA mediates selective mRNA degradation. Cell 46: 659-667.

Simopoulos, A. (1991). Omega-3 fatty acids in health and disease and in growth and development. Am. J. Clin. Nutr. 54: 438-463.

Simpson, A. E. (1997). The cytochrome P450 4 (CYP4) family. Gen Pharmacol. 28: 351359.

Sinclair, J. F., Wood, S., Lambrecht, L., Gorman, N., Mende-Mueller, L., Smith, L., Hunt, J. and Sinclair, P. R. (1990). Isolation of four forms of acetone-induced cytochrome P-450 in chicken liver by h.p.l.c. and their enzymic characterization. Biochem. J. 269: 8591.

Singer, R. H., Lawrence, J. B. and Villnave, C. (1986). Optimization of in situ hybridization using isotopic and non-isotopic detection methods. BioTechniques 4: 230250.

Sligar, S. G. and Murray, R. I. (1986). Cytochrome P450 ${ }_{\text {cam }}$ and other bacterial P-450 enzymes. Cytochrome P-450: Structure, Mechanism, and Biochemistry. New York, Plenum. 429-503.

Smith, D. A. and Jones, B. C. (1992). Speculations on the substrate structure-activity relationship (SSAR) of cytochrome P450 enzymes. 44: 2080-2098. 
Smith, P. K., Krohn, R. I., Hermanson, G. T., Mallia, A. K., Gartner, F. H., Provenzano, M. D., Fujimoto, E. K., Goeke, N. M., Olson, B. J. and Klenk, D. C. (1985). Measurement of protein using bicinchoninic acid. Anal. Biochem. 150: 76-85.

Smith, W. L. (1989). The eicosanoids and their biochemical mechanisms of action. Biochem. J. 259: 315-324.

Smith, W. L. and Dewitt, D. L. (1996). Prostaglandin endoperoxide H synthases-1 and -2. Advances in Immunology 62: 167-215.

Sogawa, K., Gotoh, O., Kawajiri, K., Harada, T. and Fujii-Kuriyama, Y. (1985). Complete nucleotide sequence of a methylcholanthrene-inducible cytochrome P-450 (P450d) gene in the rat. J. Biol. Chem. 260: 5026-5032.

Sonderfan, A. J., Arlotto, M. P. and Parkinson, A. (1989). Identification of the cytochrome P-450 isozymes responsible for testosterone oxidation in rat lung, kidney, and testis: evidence that cytochrome P-450a (P450IIA1) is the physiologically important testosterone 7a-hydroxylase in rat testis. Endocrin. 125: 857-866.

Soucek, P. and Gut, I. (1992). Cytochrome P-450 in rats: structures, functions, properties and relevant human forms. Xenobiotica 22: 83-103.

Spector, A. A., Gordon, J. A. and Moore, S. A. (1988). Hydroxyeicosatetraenoic acids (HETEs). Prog. Lipid Res. 27: 271-323.

Stanley-Samuelson, D. W. (1994). The biological significance of prostanoids and related eicosanoids in invertebrates. Amer. Zool. 34: 589-598.

Stegeman, J. J. (1987). Monooxygenase systems in marine fish. Pollutant Studies in Marine Animals. Boca Raton, Florida, CRC Press. 65-.

Stegeman, J. J. (1989). Cytochrome P450 forms in fish: catalytic, immunological and sequence similarities. Xenobiotica 19: 1093-1110.

Stegeman, J. J. (1993). Cytochrome P450 forms in fish. Cytochrome P450. SpringerVerlag.

Stegeman, J. J. (1993). The cytochromes P450 in fish. Biochemistry and molecular biology of fishes. Elsevier Science Publishers. 137-158.

Stegeman, J. J. and Hahn, M. E. (1994). Biochemistry and molecular biology of monooxygenases: Current perspectives on forms, functions and regulation of cytochrome P450 in aquatic species. Aquatic Toxicology: molecular, biochemical, and cellular perspectives. Boca Raton, Lewis. 87-206.

Stegeman, J. J., Klotz, A. V., Woodin, B. R. and Pajor, A. M. (1981). Induction of hepatic cytochrome P-450 in fish and the indication of environmental induction in scup. Aquat. Toxicol. 1: 197-212. 
Stegeman, J. J., Renton, K. W., Woodin, B. R., Zhang, Y.-S. and Addison, R. F. (1990). Experimental and environmental induction of cytochrome P450E in fish from Bermuda waters. J. Exp. Mar. Biol. Ecol. 138: 49-67.

Stegeman, J. J. and Woodin, B. R. (1988). Cytochromes P-450 in fish from Bermuda: Evidence for induction by environmental chemicals and for the presence of forms related to mammalian PB-inducible P-450IIB. Poster presented at the New England Membrane Enzyme Group meetings.

Stegeman, J. J., Woodin, B. R. and Goksøyr, A. (1988). Apparent cytochrome P-450 induction as an indication of exposure to environmental chemicals in the flounder Platichthys flesus. Mar. Ecol. Prog. Ser. 46: 55-60.

Stegeman, J. J., Woodin, B. R., Park, S. S., Kloepper-Sams, P. J. and Gelboin, H. V. (1985). Microsomal cytochrome P-450 function in fish evaluated with polyclonal and monoclonal antibodies to cytochrome P-450E from scup (Stenotomus chrysops). Mar. Environ. Res. 17: 83-86.

Stegeman, J. J., Woodin, B. R. and Waxman, D. J. (1990). Structural relatedness of mammalian cytochromes P450 IIB and cytochrome P450B from the marine fish scup (Stenotomus chrysops). FASEB J 4: A739.

Stien, X., Amichot, M., Berge, J.-B. and Lafaurie, M. (1995). Molecular clonig of a CYP1A cDNA from the teleost fish Dicentrarchus labrax. Unpublished

Sutter, T. R., Tang, Y. M., Hayes, C. L., Wo, Y.-Y. P., Jabs, E. W., Li, X., Yin, H., Cody, C. W. and Greenlee, W. F. (1994). Complete cDNA sequence of a human dioxininducible mRNA identifies a new gene subfamily of cytochrome P450 that maps to chromosome 2. J. Biol. Chem. 269: 13092-13099.

Suwa, Y., Mizukami, Y., Sogowa, K. and Fujii-Kuriyama, Y. (1985). Gene structure of a major phenobarbital-inducible cytochrome P450 in rat liver. J. Biol. Chem. 260: 79807984.

Swofford, D. L. (1990). PAUP: Phylogenetic Analysis Using Parsimony. Champaign, IL, Illinois Natural History Survey.

Swofford, D. L., Olsen, G. J., Waddell, P. J. and Hillis, D. M. (1996). Phlogenetic Inference. Molecular Systematics. Sunderland, Sinauer Associates, Inc. 407-514.

Takahashi, M., Tanaka, M., Sakai, N., Adachi, S., Miller, W. L. and Nagahama, Y. (1993). Rainbow Trout Ovarian Cholesterol Side-Chain Cleavage Cytochrome-P450 (P450scc) - cDNA Cloning and Messenger RNA Expression During Oogenesis. FEBS Lett 319: 45-48.

Tanaka, M., Fukada, S., Matsuyama, M. and Nagahama, Y. (1995). Structure and promoter analysis of the cytochrome P-450 aromatase gene of the teleost fish, medaka (Orysias latipes). J. Biochem. 117: 719-725. 
Tanaka, M., Telecky, T. M., Fukuda, S., Adachi, S. and Chen, S. (1992). Cloning and sequence analysis of the cDNA encoding P-450 aromatase (P450arom) from a rainbow trout (Oncorhynchus mykiss) ovary: relationship between the amount of P450arom mRNA and the production of oestradiol-17b in the ovary. J. Mol. Endocrinol. 8: 53-61.

Tanaka N., K. T., Taniguchi T. (1993). Recognition DNA sequences of interferon regulatory

factor 1 (IRF-1) and IRF-2, regulators of cell growth

and the interferon system. Mol. Cell. Biol. 13: 4531-4538.

Trant, J. (1994). Isolation and characterization of the cDNA encoding the channel catfish (Ictalurus punctatus) form of cytochrome P450arom. Gen. Comp. Endocrinol. 95: 155168.

Trant, J. M. (1995). Isolation and characterization of the cDNA encoding the spiny dogfish shark (Squalus acanthias) form of cytochrome P450c17. Journal of Experimental Zoology 272: 25-33.

Valiela, I., Wright, J., Teal, J. and Volkmann, S. (1977). Growth, production and energy transformations in the salt-marsh killifish Fundulus heteroclitus. Marine Bio. 40: 135-144.

VanRollins, M. (1990). Synthesis and characterization of cytochrome P-450 epoxygenase metabolites of eicosapentaenoic acid. Lipids 25: 481-490.

Verkade, P. E. and Lee, J. v. d. (1934). Researches on fat metabolism. Biochem. J. 28: 31-40.

Vrolijk, N. H., Lin, C. and Chen, T. T. (1995). Characterization and expression of a CYP1A gene from the tropical teleost, Chaetodon capistratus. Unpublished

Vrolijk, N. H. and Targett, N. M. (1991). Comparison of in vivo detoxification enzyme activities among three members of the genus Chaetodon (butterflyfish). SECOND INTERNATIONAL MARINE BIOTECHNOLOGY CONFERENCE (IMBC' '91),

Vrolijk, N. H., Targett, N. M., Woodin, B. R. and Stegeman, J. J. (1994). Toxicological and ecological implications of biotransformation enzymes in the tropical teleost Chaetodon capistratus. Mar. Biol. 119: 151-158.

Wade, M. G. and Van Der Kraak, G. (1993). Arachidonic acid and prostaglandin E sub(2) stimulate testosterone production by goldfish testis in vitro. Gen. Comp. Endocrinol. 90: 109-118.

Wahle, E. and Walter, K. (1992). The biochemistry of 3'-end cleavage and polyadenylation of messenger RNA precoursors. Annu. Rev. Biochem. 61: 419-440.

Wakabayashi, K. and Shimazono, N. (1961). Studies in vitro on the mechanism of woxidation of fatty acids. Bichim. Biophys. Acta 48: 615-617. 
Wales, N. A. M. (1988). Hormone studies in Myxine glutinosa: effects of the eicosanoids arachidonic acid, prostaglandin E1, E2, A2, F2a, and the thromboxane B2, and of indomethacin on plasma cortisol, blood pressure, urine flow and electrolyte balance. $J$. Comp. Physiol. 158: 621-626.

Warner, M. (1982). Catalytic activity of partially purified renal 25 -hydroxyvitamin D hydroxylases from vitamin D-deficient and vitamin D-replete rats. J. Biol. Chem. 257: 12995-13000.

Warner, M., Stromstedt, M., Wyss, A. and Gustafsson, J. A. (1993). Regulation of Cytochrome P450 in the Central Nervous System. J Steroid Biochem Mol Biol 47: 191194.

Waxman, D., Dannan, G. A. and Guengerich, F. P. (1985). Regulation of rat hepatic cytochrome P-450: Age-dependent expression, hormonal imprinting, and xenobiotic inducibility of sex-specific isoenzymes. Biochemisty 24: 4409-4417.

Waxman, D. J. (1988). Interactions of hepatic cytochromes P-450 with steroid hormones. Regioselectivity and stereospecificity of steroid metabolism and hormonal regulation of rat P-450 enzyme expression. Biochem. Pharm. 37: 71-84.

Waxman, D. J., Lapenson, D. P., Aoyama, T., Gelboin, H., Gonzalez, F. J. and Korzekwa, K. (1991). Steroid hormone hydroxylase specifities of eleven cDNA-expressed human cytochrome P450s. Arch. Biochem. Biophys. 290: 160-166.

Waxman, D. J., Morrissey, J. J. and LeBlanc, G. A. (1989). Hypophysectomy differentially alters P-450 protein levels and enzyme activities in rat liver: Pituitary control of hepatic NADPH cytochrome P-450 reductase. Mol. Pharmac. 35: 519-525.

Weber, P. C. (1990). The modification of the arachidonic acid cascade by $n-3$ fatty acids. Advances in prostaglandin, thromboxane, and leukotriene research. New York, Raven Press. 232-240.

White, J. A., Guo, Y.-D., Baetz, K., Beckett-Jones, B., Bonasoro, J., Hsu, K. E., Dilworth, F. E., Jones, G. and Petkovich, M. (1996). Identification of the retinoic acidinducible all trans retinoic acid 4-hydroxylase. J. Biol. Chem. 271: 29922-29927.

White, R. D., Shea, D., Solow, A. R. and Stegeman, J. J. (1997). Induction and posttranscriptional suppression of hepatic cytochrome P4501 A1 by 3,3',4,4'tetrachlorobiphenyl. Biochem. Pharmacol. 53: 1029-1040.

Williams, C. S. and DuBois, R. N. (1996). Prostaglandin endoperoxide synthase: why two isoforms? J. Amer. Physiol. G393-G400.

Williams, D. E., Okita, R. T., Buhler, D. R. and Masters, B. S. (1984). Regiospecific hydroxylation of lauric acid at the (omega-1) position by hepatic and kidney microsomal cytochromes P-450 from rainbow trout. Arch. Biochem. Biophys. 231: 503-510.

Wolf, C. R., Palmer, J. F., Hughes, V. and English, N. T. (1996). Fatty acids as transcriptional regulators of gene expression. XIth international symposium on microsomes and drug oxidations, Los Angles, 
Woodin, B. R., Gooch, J. W., Gooch, C. G. and Stegeman, J. J. (1988). Cross reactivity of monoclonal antibodies to scup cytochrome P450A and P450B. New England Membrane Enzyme Group.

Wu, S., Moomaw, C. R., Tomer, K. B., Falck, J. R. and Zeldin, D. C. (1996). Molecular cloning and expression of CYP2J2, a human cytochrome P450 arachidonic acid epoxygenase highly expressed in heart. J. Biol. Chem. 271: 3460-3468.

Wu, S., Weina, C., Murphy, E., Gabel, S., Tomer, K. B., Foley, J., Steenbergen, C., Falck, J. R., Moomaw, C. R. and Zeldin, D. C. (1997). Molecular cloning, expression, and functional significance of a cytochrome $\mathrm{P} 450$ highly expressed in rat heart myocytes. Journal of Biological Chemistry 1-9.

Yamawaki, M., Zurbriggen, A., Richard, A. and Vanvelde, M. (1993). Saponin treatment for in situ hybridization maintains good morphological preservation. Journal of Histochemistry and Cytochemistry 4: 105-109.

Yamazaki, S., Sato, K., Suhara, K., Sakaguchi, M., Mihara, K. and Omura, T. (1993). Importance of the proline-rich region following signal-anchor sequence in the formation of correct conformation of microsomal cytochrome P-450s. J. Biochem. 114: 652-657.

Yang, Y., Wang, J. and Buhler, D. R. (1995). Cloning and expression of CYP2M1, a new cytochrome P450 isoform from rainbow trout liver. Arch. Biochem. Biophys. in press.

Yang, Y., Wang, J. and Buhler, D. R. (1996). Cloning and characterization of a new cytochrome P450 from rainbow trout. Marine Environ. Res. 42: 27.

Yun, C.-H., Shimada, T. and Guengerich, F. P. (1991). Purification and characterization of human liver microsomal cytochrome p__450 2A6. Mol. Pharmacol. 40: 679-685.

Zabelinskii, S. A., Brovtsyna, N. B., Chebotareva, M. A., Gorbunova, O. B. and Krivchenko, A. I. (1995). Comparative investigation of lipid and fatty acid composition of fish gills and mammalian lungs. A model of the membrane component areas. Comp.

Biochem. Physiol. 111B: 127-140.

Zacharewski, T. R., McCallum, G. P., Stegeman, J. J. and Bend, J. R. (1996). Hepatic cytochrome P450 1A as a biomarker for hydrocarbon and oxidant pollutants in fish: Relevance of identification of specific mechanism(s) of induction and potential effects on homeostasis. Environmental Toxicology and Risk Assessment: Transboundary Issues in Pollution-Air, Surface and Groundwater. Philadelphia, American Society for Testing and Materials. 165-187.

Zeldin, D., Foley, J., Goldsworthy, S., Cook, M., Boyle, J., Ma, J., Moomaw, C., Tomer, K., Steenbergen, C. and Wu, S. (1997). CYP2J subfamily P450s in the gastrointestinal tract: expression, localization, and potential functional significance. Molecular Pharm. 51: 931-43. 
Zeldin, D. C., Plitman, J. D., Kobayashi, J., Miller, R. F., Snapper, J. R., Falck, J. R., Szarek, J. L., Philpot, R. M. and Capdevila, J. H. (1995). The rabbit pulmonary cytochrome $\mathrm{P} 450$ arachidonic acid metabolic pathway: Characterization and significance. $J$. Clin. Invest. 95: 2150-2160.

Zeldin, D. C., Wu, S. and Ma, J. (in press). CYP2J subfamily P450s: physiologically relevant hemoproteins active in the metabolism of arachidonic acid in hepatic and extrahepatic tissues. Reviews in Toxicology

Zhang, Q.-Y., Ding, X. and Kaminsky, L. S. (1997). cDNA cloning, heterologous expression, and characterization of rat intestinal CYP2J4. Arch. Biochem. Biophys.

Zimniak, P. and Waxman, D. J. (1993). Liver cytochrome P450 metabolism of endogenous steroid hormones, bile acids, and fatty acids. Cytochrome P450 Handbook of Experimental Pharmacology. Springer-Verlag. 123-144.

Zou, A. P., Fleming, J. T., Falck, J. R., Jacobs, E. R., Gebremedhin, D., Harder, D. R. and Roman, R. J. (1994). Regional and stereospecific effects of 11,12-epoxyeicosatrienoic acids on vascular tone and $\mathrm{K}+$ channel activity in renal arteries (abstract). Hypertension 24 : 382 . 